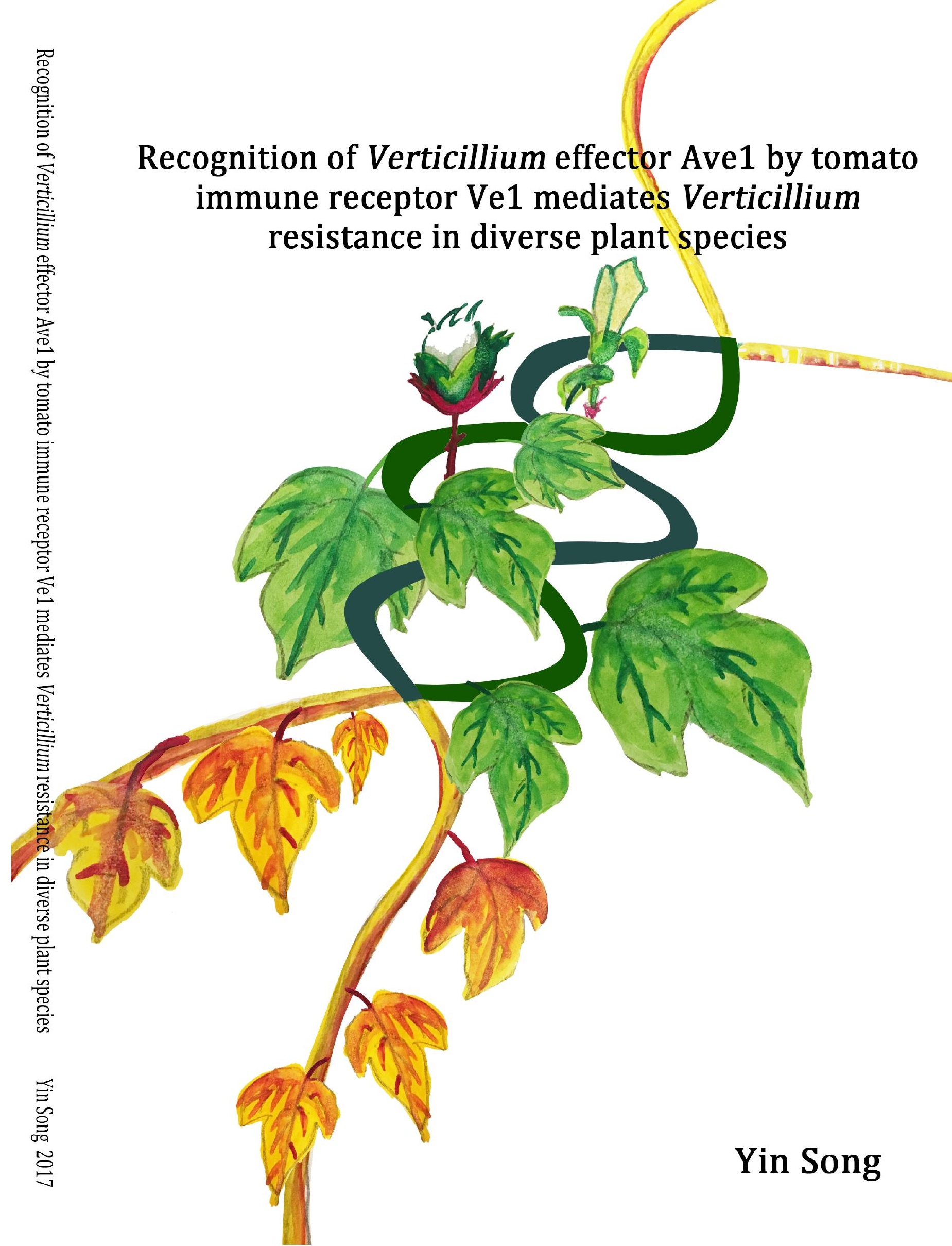




\section{Propositions}

1. Tomato Ve1 has traits of a typical race-specific resistance (R) protein as well as of a typical pattern recognition receptor (PRR). (this thesis)

2. The modular composition of immune receptors offers opportunities to design tailored immune receptors. (this thesis)

3. For better understanding of biological systems, the massive amounts of genomic DNA sequence that are generated by nextgeneration sequencing technologies are useless without further efforts to precisely assemble and annotate genomes.

4. Challenging entrenched scientific beliefs can lead to groundbreaking research.

5. As much as how a critical spirit can improve the quality of science, it can simultaneously impair the quality of life.

6. In the age of e-commerce scientists need to be salespersons.

Propositions belonging to the thesis, entitled

"Recognition of Verticillium effector Ave1 by tomato immune receptor Ve1 mediates Verticillium resistance in diverse plant species"

Yin Song

Wageningen, 23 October 2017 


\section{Recognition of Verticillium effector Ave1 by tomato immune receptor Ve1 mediates Verticillium resistance in diverse plant species}

Yin Song 


\section{Thesis committee}

\section{Promotors}

Prof. Dr B.P.H.J. Thomma

Professor of Phytopathology

Wageningen University \& Research

Prof. Dr P.J.G.M. de Wit

Professor of Phytopathology

Wageningen University \& Research

\section{Other members:}

Prof. Dr T. Bisseling, Wageningen University \& Research

Dr G. Smant, Wageningen University \& Research

Prof. Dr J. Schirawski, RWTH Aachen University, Germany

Dr V.G.A.A. Vleeshouwers, Wageningen University \& Research

This research was conducted under the auspices of the Graduate School of Experimental Plant Sciences 


\title{
Recognition of Verticillium effector Ave1 by tomato immune receptor Ve1 mediates Verticillium resistance in diverse plant species
}

\section{Yin Song}

\author{
Thesis \\ submitted in fulfilment of the requirements for the degree of doctor \\ at Wageningen University \\ by the authority of the Rector Magnificus, \\ Prof. Dr A.P.J. Mol, \\ in the presence of the \\ Thesis Committee appointed by the Academic Board \\ to be defended in public \\ on Monday 23 October 2017 \\ at 1:30 p.m. in the Aula.
}




\section{Yin Song}

Recognition of Verticillium effector Ave1 by tomato immune receptor Ve1 mediates Verticillium resistance in diverse plant species,

232 pages.

PhD thesis, Wageningen University, Wageningen, the Netherlands (2017)

With references, with summary in English

ISBN: 978-94-6343-795-0

DOI: https://doi.org/10.18174/423469 


\section{Contents}

$\begin{array}{lll}\text { Chapter } 1 & \text { General introduction } & 7\end{array}$

$\begin{array}{lll}\text { Chapter } 2 \text { Biological functions of effectors secreted by soil-borne } & 19\end{array}$ plant pathogens

$\begin{array}{lll}\text { Chapter } 3 \text { Tomato immune receptor Ve1 recognizes co-localized N- } & 47\end{array}$ and C-termini of Verticillium dahliae effector Ave1

Chapter 4 Broad taxonomic characterization of Verticillium wilt resistance genes reveals ancient origin of the tomato Ve1 immune receptor

Chapter 5 Transfer of tomato immune receptor Ve1 confers Ave1129 dependent Verticillium resistance in tobacco and cotton

Chapter 6 Host-induced gene silencing compromises Verticillium wilt in tomato and Arabidopsis

Chapter 7 General discussion

Summary

Acknowledgements

Curriculum vitae

List of publications 

Chapter 1

General introduction 


\section{Chapter 1}

\section{INTRODUCTION}

Plants are constantly engaged in battles against a wide range of potential pathogens within their environment. Nevertheless, only few of these potential pathogens succeed in invading a plant to cause disease, as plants have evolved innate immunity to protect themselves against microbial attack (Dodds and Rathjen, 2010; Thomma et al., 2011). Plant innate immunity against pathogen attack is governed by immune receptors that sense pathogen(-induced) ligands to activate defense. Originally, the interaction between plant immune receptors and pathogen ligands was described as a "gene-for-gene" model, stating that the products of plant resistance $(R)$ genes induce race-specific resistance upon recognition of the products of corresponding pathogen avirulence (Avr) genes (Flor, 1971). Decades later, an updated view of plant innate immunity has been introduced as the "zigzag" model, by incorporating pathogen-secreted effector molecules that suppress host immune responses, but that may subsequently be recognized by newly evolved immune receptors, in turn (Jones and Dangl, 2006). In this model, the first layer of defense is governed by plasma membrane-localized pattern recognition receptors (PRRs) that detect conserved microbe-associated molecular patterns (MAMPs) to activate MAMP-triggered immunity (MTI). In subsequent layers of defense effectors are recognized by corresponding resistance proteins ( $\mathrm{R}$ proteins), resulting in effector-triggered immunity (ETI). Although initially portrayed as separate layers of defense, numerous studies on various plant-microbe interactions have revealed that the delineation between MTI and ETI is not strict, but rather a continuum (Thomma et al., 2011). Moreover, the conceptual conflict that MAMPs are defined from the perspective of the host whereas effectors are defined from the perspective of the invader has recently inspired the proposal of the Invasion Model, in which host receptors (termed invasion pattern receptors; IPRs) detect either an externally encoded or modified-self ligand that indicates invasion (termed invasion patterns; IPs) (Cook et al., 2015). In this model, any molecule can serve as an IP that is potentially detected by an IPR, but the probability of a particular ligand-receptor complex to evolve within the framework of host immunity increases with increasing ligand probability to retain function, conservation across organisms, importance in establishment of symbiosis, and accessibility. 
Verticillium is a relatively small genus of ascomycete fungi that currently comprises ten species (Inderbitzin et al., 2011; Figure 1). All presently recognized Verticillium species are soil-borne fungi, and several of them cause so-called vascular wilt diseases in a wide range of economically important crops, including tomato, potato, tobacco, cotton, hop plants, lettuce, olive trees, oilseed rape, sunflower, sugar beet etc (Pegg and Brady, 2002; Figure 2). Although symptoms may vary considerably between plant hosts, the most frequently observed symptoms of Verticillium wilt include stunting, wilting, chlorosis, necrosis, vascular discoloration and early senescence (Fradin and Thomma, 2006). Within the Verticillium genus, $V$. dahliae is the most notorious species that can cause Verticillium wilt diseases in over 200 plant host species worldwide. V. albo-atrum, V. longisporum, $V$. alfalfae and $V$. non-alfalfae are also economically important vascular pathogens, albeit with narrower host ranges (Pegg and Brady, 2002; Agrios, 2005; Fradin and Thomma, 2006; Klosterman et al., 2009; Inderbitzin et al., 2011; Depotter et al., 2015). Polygenic resistance to Verticillium spp. has been described in several plant species, including potato, hop, alfalfa, cotton and strawberry (Simko et al., 2004; Bolek et al., 2005; Wang et al., 2008; Yang et al., 2008; Jakse et al., 2013; Antanaviciute et al., 2015), whereas single dominant resistance genes have been identified in tomato, potato and lettuce species only (Schaible et al., 1951; Simko et al., 2004; Hayes et al., 2011; Christopoulou et al., 2015). In tomato (Solanum lycopersicum), a single dominant locus that confers Verticillium resistance has been identified as the Ve locus, which controls Verticillium isolates that are assigned to race 1, but not to race 2 (Schaible et al., 1951; Pegg, 1974). This locus contains two closely linked and inversely oriented genes, $V e 1$ and $V e 2$, that both encode extracellular leucine rich repeat (eLRR) receptor-like proteins (RLPs) (Kawchuk et al., 2001; Wang et al., 2010). Of these, only Ve1 was found to act as a functional $V$. dahliae resistance gene in tomato (Fradin et al., 2009). Interestingly, interfamily transfer of Ve1 from tomato to Arabidopsis resulted in Verticillium resistance in the latter species (Fradin et al., 2011, 2014; Zhang et al., 2014), implying that the underlying immune signalling pathway is conserved (Fradin et al., 2011; Thomma et al., 2011). So far, several Ve1 homologs were identified within the Solanaceae family (in wild relatives of tomato and eggplant and in cultivated potato; Chai et al., 2003; Fei et al., 2004; Simko et al., 2004; Liu et al., 2012), as well as outside this 
family (mint, lettuce, cotton and grape; Vining and Davis, 2009; Hayes et al., 2011; Zhang et al., 2011; Zhang et al., 2012; Yang et al., 2014; Chen et al., 2016; Chen et al., 2017). Through comparative population genomics, the $V$. dahliae effector protein that is recognized by the tomato Ve1 immune receptor was identified as Ave1 (for

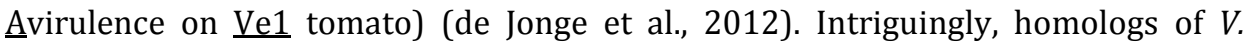
dahliae Ave1 (VdAve1) were identified in a number of plant pathogenic microbes,

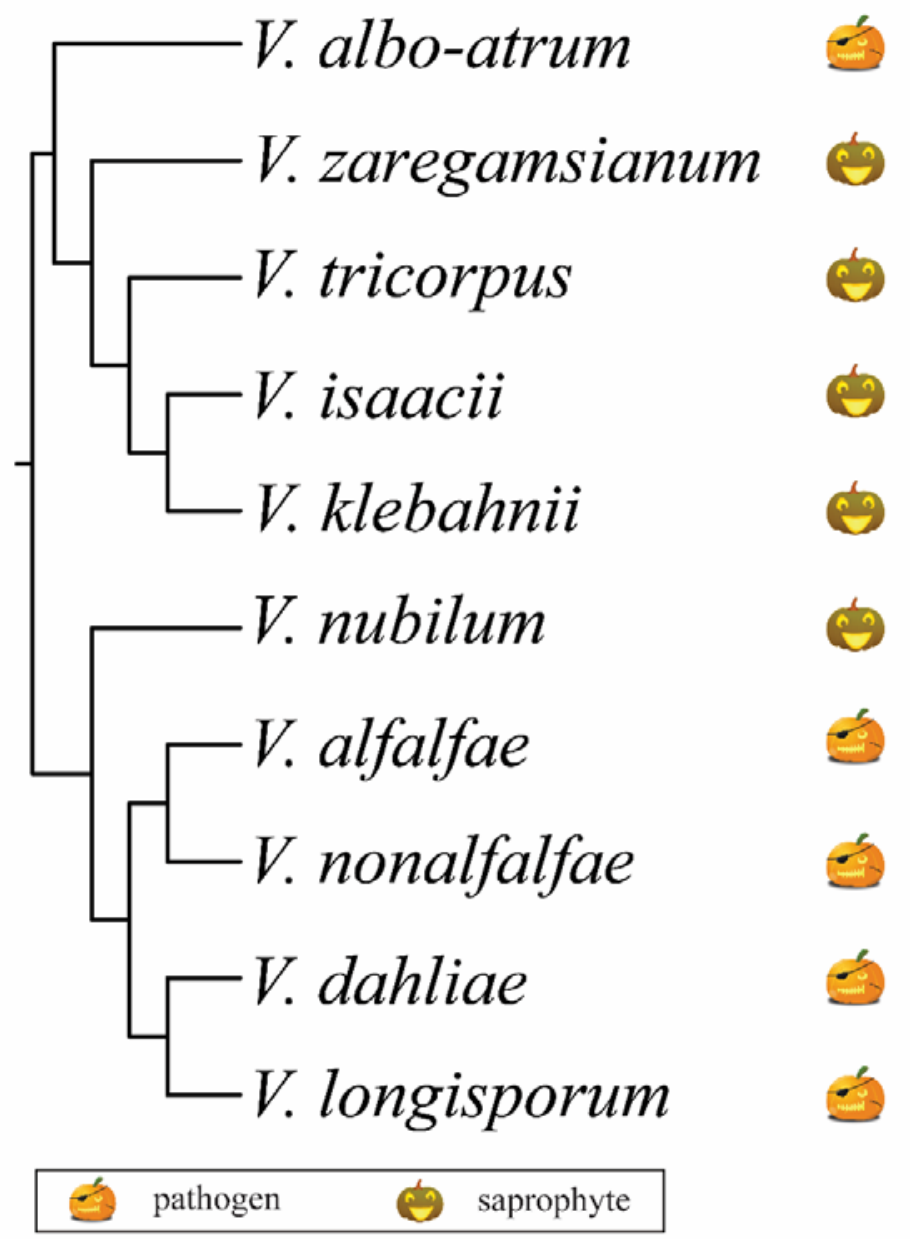

Figure 1. Phylogeny of species within the Verticillium genus. The phylogenetic tree of Verticillium species is adapted from Shi-Kunne et al. (unpublished data). Verticillium species marked on the right by a pirate pumpkin are pathogenic fungi on plants, while Verticillium species marked on the right by a smiling pumpkin are saprophytes. 


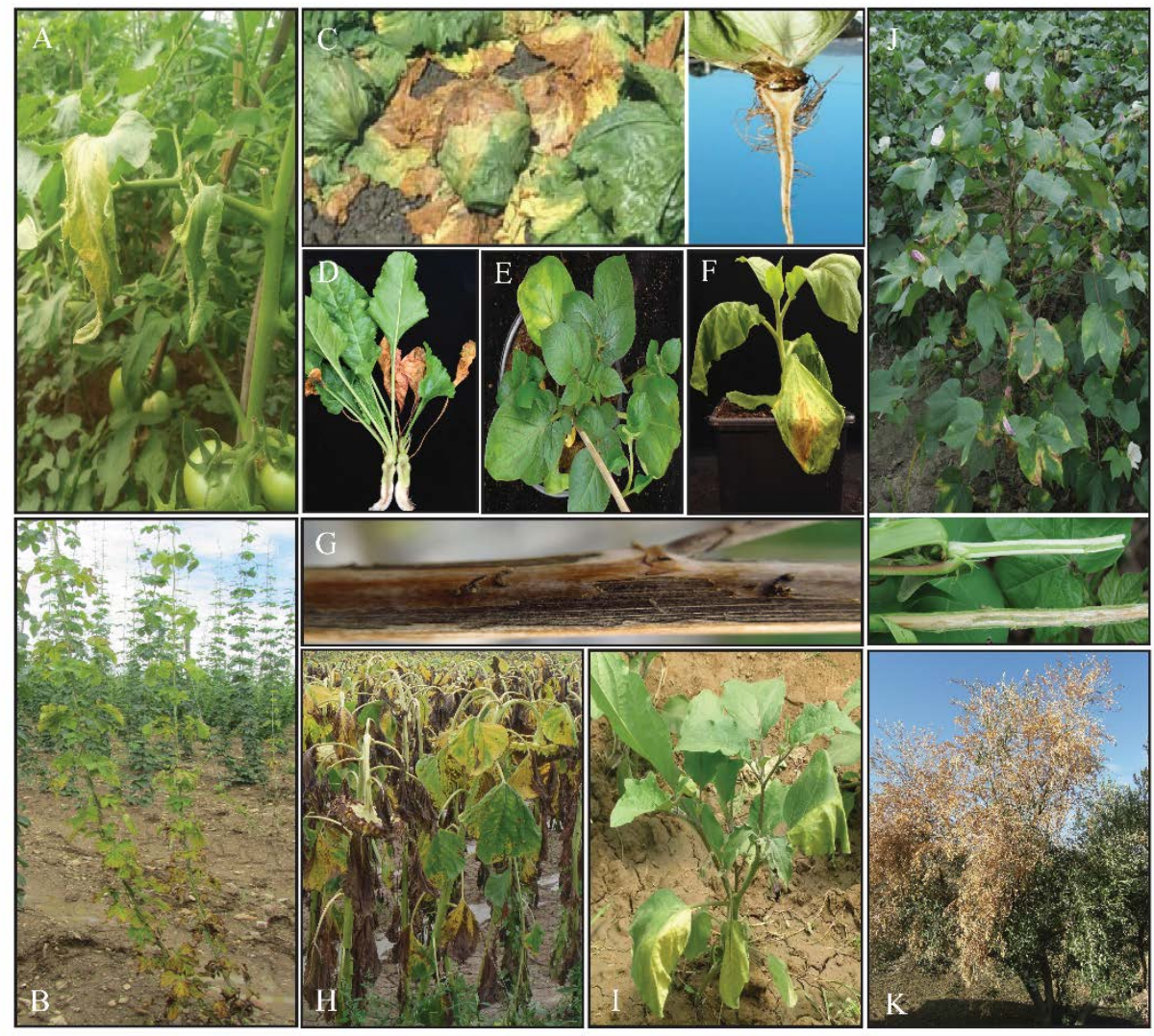

Figure 2 Disease symptoms on various host plants caused by various Verticillium species. A, C-E, H-K, Verticillium wilt symptoms caused by V. dahliae on tomato (A), lettuce (C), sugar beet (D), potato (E), sunflower (H), eggplant (I), cotton (J) and olive tree (K). B, Verticillium wilt of hop plants caused by V. nonalfalfae. F, Disease caused by V. alfalfae on tobacco. G, Stem striping caused by V. longisporum on oilseed rape. Photos are courtesy of Sebastjan Radišek and Branka Javornik (panel B), Krishna Subbarao (panel C), Jasper Depotter (panel G), Shuqing Yang and Jun Zhao (panel H), Longfu Zhu (panel J) and Jelle Hiemstra (panel $\mathbf{K}$ ).

including the citrus bacterial canker pathogen Xanthomonas. axonopodis pv. citri (XacPNP), the fungal tomato wilt pathogen Fusarium oxysporum f. sp. lycopersici (FoAve1), the fungal sugar beet leaf spot pathogen Cercospora beticola (CbAve1), the crucifer anthracnose pathogen Colletotrichum higginsianum (ChAve1) and the cucurbit anthracnose pathogen Colletotrichum orbiculare (CoAve1) (Nembaware et al., 2004; de Jonge et al., 2012; Gan et al., 2013), and a few of these homologs are differentially recognized by tomato Ve1 (de Jonge et al., 2012). Consequently, Ve1 


\section{Chapter 1}

was found to mediate resistance towards F. oxysporum in tomato, demonstrating involvement of this tomato immune receptor in resistance against multiple fungal pathogens (de Jonge et al., 2012).

\section{MAIN OBJECTIVE AND RESEARCH QUESTIONS}

The main objective of my $\mathrm{PhD}$ research was to characterize the role of the immune receptor Ve1 in tomato and its homologs in various plant species in Verticillium resistance, and to mechanistically unravel the functionality of tomato Ve1. To this end, I have addressed the following research questions:

i. Can we identify a minimal motif in the effector VdAve1 that is required for its recognition by tomato immune receptor Ve1?

ii. Do homologs of tomato Ve1 that are found in other plant species similarly act as immune receptors that govern resistance against race 1 strains of $V$. dahliae in a VdAve1-dependent manner?

iii. Does ectopic expression of tomato Ve1 in crop species confer VdAve1-dependent Verticillium resistance?

\section{THESIS OUTLINE}

Research on effectors secreted by pathogens during host colonization has dominated the field of molecular plant-microbe interactions over recent years. Chapter 2 reviews the current knowledge on how soil-borne pathogens employ secreted effector molecules to support host colonization. Whereas most wellstudied effectors are proteinaceous molecules, non-canonical effectors, such as metabolites and small RNAs, have also been described. Soil-borne pathogens live in a complex and sophisticated belowground environment. In addition to attenuation of host immunity and roles in self-defense, the potential role of effectors in interactions with other microbiome inhabitants, including competitors and mycoparasites, is discussed.

Effectors are molecules secreted by plant pathogens to facilitate infection, often through deregulation of plant immune responses. During host colonization, race 1 strains of $V$. dahliae secrete the effector protein VdAve1 that activates Ve1mediated immunity. Homologs of VdAve1 were identified in the bacterial plant 
pathogen $X$ axonopodis pv. citri (XacPNP) and in the plant pathogenic fungi $F$. oxysporum f. sp. lycopersici (FoAve1), C. beticola (CbAve1), and C. higginsianum (ChAve1). These homologs are differentially recognized by Ve1, with the most efficient recognition of VdAve1 and no recognition of XacPNP. Chapter 3 aims to identify a minimal motif of VdAve1 that is required to activate Ve1-mediated immunity. The approach was based on epitope prediction, guided by the alignment of differentially recognized VdAve1 homologs, followed by functional analyses of a combination of serial deletions assays, domain swaps, synthetic peptides, threedimensional structural prediction and chimeric proteins.

So far, several Ve1 homologs were identified within and outside the Solanaceae family. However, functionality of these homologs against Verticillium wilt remained obscure. In Chapter 4, I report the cloning and functional characterization of Ve1 homologs from tobacco (Nicotiana glutinosa), potato (Solanum tuberosum), wild eggplant (Solanum torvum) and hop (Humulus lupulus).

The tomato immune receptor Ve1 recognizes VdAve1 and its homologs from other pathogenic microbes including the tomato pathogen $F$. oxysporum $\mathrm{f}$. sp. lycopersici (FoAve1) and the sugar beet pathogen C. beticola (CbAve1). Previous work revealed that tomato Ve1 mediates resistance against multiple fungal tomato pathogens including $V$. dahliae, V. albo-atrum and F. oxysporum, and transgenic expression of tomato $V e 1$ in Arabidopsis confers Verticillium resistance through recognition of VdAve1. In Chapter 5 I investigated whether the tomato immune receptor gene Ve1 can confer Verticillium wilt resistance when transferred from tomato to the closely related crop species tobacco (Nicotiana tobacum cv. Samsun) and the distantly related crop species cotton (Gossypium hirsutum). The Ve1transgenic lines were subjected to molecular characterization and disease assays using race 1 Verticillium strains.

Previous studies have shown that the expression of silencing constructs in host plants designed to target transcripts of pathogen genes can specifically silence these genes in invading pathogens, including fungi, which is referred as hostinduced gene silencing (HIGS). HIGS was shown to operate against the fungal pathogens Blumeria graminis, Puccinia spp., Fusarium spp., Sclerotinia sclerotiorum and Rhizoctonia solani. In Chapter 6 I assessed the effect of HIGS on Verticillium wilt disease. This was done by transient assays in tomato based on virus-induced 
Chapter 1

gene silencing, followed by experiments with stable transgenic Arabidopsis thaliana plants expressing hairpin constructs targeting transcripts of three $V$. dahliae genes.

In the final chapter of the thesis, Chapter 7, the major results described in the previous chapters are discussed and placed in a broader perspective. 


\section{REFERENCES}

Agrios G (2005) Plant Pathology, $5^{\text {th }}$ edn. Elsevier Academic Press, New York.

Antanaviciute L, Šurbanovski N, Harrison N, McLeary K, Simpson D, Wilson F, Sargent D, Harrison R (2015) Mapping QTL associated with Verticillium dahliae resistance in the cultivated strawberry (Fragaria $\times$ ananassa). Hortic Res 2: 15009.

Bolek Y, El-Zik KM, Pepper AE, Bell AA, Magill CW, Thaxton PM, Reddy OUK (2005) Mapping of verticillium wilt resistance genes in cotton. Plant Sci 168: 1581-1590.

Chai Y, Zhao L, Liao Z, Sun X, Zuo K, Zhang L, Wang S, Tang K (2003) Molecular cloning of a potential Verticillium dahliae resistance gene SIVe1 with multi-site polyadenylation from Solanum licopersicoides. DNA Seq 14: 375-384.

Chen J-Y, Li N-Y, Ma X-F, Gupta VK, Zhang D-D, Li T-G, Dai X-F (2017) The ectopic overexpression of the cotton $\mathrm{Ve} 1$ and $V e 2$-homolog sequences leads to resistance response to Verticillium wilt in Arabidopsis. Front Plant Sci 8: 844.

Chen T, Kan J, Yang Y, Ling X, Chang Y, Zhang B (2016) A Ve homologous gene from Gossypium barbadense, Gbvdr3, enhances the defense response against Verticillium dahliae. Plant Physiol Biochem 98: 101-111.

Christopoulou M, Wo SR-C, Kozik A, McHale LK, Truco M-J, Wroblewski T, Michelmore R (2015) Genome-wide architecture of disease resistance genes in lettuce. G3 5: 2655-2699.

Cook DE, Mesarich CH, Thomma BPHJ (2015) Understanding Plant immunity as a surveillance system to detect invasion. Annu Rev Phytopathol 53: 541-563.

de Jonge R, van Esse HP, Maruthachalam K, Bolton MD, Santhanam P, Saber MK, Zhang Z, Usami T, Lievens B, Subbarao KV, Thomma BPHJ (2012) Tomato immune receptor Ve1 recognizes effector of multiple fungal pathogens uncovered by genome and RNA sequencing. Proc Natl Acad Sci USA 109: 5110-5115.

Depotter JR, Deketelaere S, Inderbitzin P, von Tiedemann A, Höfte M, Subbarao KV, Wood TA, Thomma BPHJ (2015) Verticillium longisporum, the invisible threat to oilseed rape and other Brassicaceous plant hosts. Molecular plant pathology

Dodds PN, Rathjen JP (2010) Plant immunity: towards an integrated view of plant-pathogen interactions. Nat Rev Genet 11: 539-548.

Fei J, Chai Y, Wang J, Lin J, Sun X, Sun C, Zuo K, Tang K (2004) cDNA cloning and characterization of the Ve homologue gene StVe from Solanum torvum Swartz. DNA Seq 15: 88-95.

Flor HH (1971) Current status of the gene-for-gene concept. Annu Rev Phytopathol 9: 275-296.

Fradin EF, Abd-El-Haliem A, Masini L, van den Berg GC, Joosten MH, Thomma BPHJ (2011) Interfamily transfer of tomato Ve1 mediates Verticillium resistance in Arabidopsis. Plant Physiol 156: 2255-2265.

Fradin EF, Thomma BPHJ (2006) Physiology and molecular aspects of Verticillium wilt diseases caused by V. dahliae and V. albo-atrum. Mol Plant Pathol 7: 71-86.

Fradin EF, Zhang Z, Ayala JCJ, Castroverde CD, Nazar RN, Robb J, Liu C-M, Thomma BPHJ (2009) Genetic dissection of Verticillium wilt resistance mediated by tomato Ve1. Plant Physiol150: 320332.

Fradin EF, Zhang Z, Rovenich H, Song Y, Liebrand TW, Masini L, van den Berg GC, Joosten MH, Thomma BPHJ (2014) Functional analysis of the tomato immune receptor Ve1 through domain swaps with its non-functional homolog Ve2. PLOS ONE 9: e88208. 


\section{Chapter 1}

Gan P, Ikeda K, Irieda H, Narusaka M, O'Connell RJ, Narusaka Y, Takano Y, Kubo Y, Shirasu K (2013) Comparative genomic and transcriptomic analyses reveal the hemibiotrophic stage shift of Colletotrichum fungi. New Phytol 197: 1236-1249.

Hayes RJ, McHale LK, Vallad GE, Truco MJ, Michelmore RW, Klosterman SJ, Maruthachalam K, Subbarao KV (2011) The inheritance of resistance to Verticillium wilt caused by race 1 isolates of Verticillium dahliae in the lettuce cultivar La Brillante. Theor Appl Genet 123: 509-517.

Inderbitzin P, Bostock RM, Davis RM, Usami T, Platt HW, Subbarao KV (2011) Phylogenetics and taxonomy of the fungal vascular wilt pathogen Verticillium, with the descriptions of five new species. PLOS ONE 6: e28341.

Inderbitzin P, Davis RM, Bostock RM, Subbarao KV (2011) The ascomycete Verticillium longisporum is a hybrid and a plant pathogen with an expanded host range. PLOS ONE 6: e18260.

Jakse J, Cerenak A, Radisek S, Satovic Z, Luthar Z, Javornik B (2013) Identification of quantitative trait loci for resistance to Verticillium wilt and yield parameters in hop (Humulus lupulus L.). Theor Appl Genet 126: 1431-1443.

Jones JD, Dangl JL (2006) The plant immune system. Nature 444: 323-329.

Kawchuk LM, Hachey J, Lynch DR, Kulcsar F, van Rooijen G, Waterer DR, Robertson A, Kokko E, Byers R, Howard RJ (2001) Tomato Ve disease resistance genes encode cell surface-like receptors. Proc Natl Acad Sci USA 98: 6511-6515.

Klosterman SJ, Atallah ZK, Vallad GE, Subbarao KV (2009) Diversity, pathogenicity, and management of Verticillium species. Annu Rev Phytopathol 47: 39-62.

Liu SP, Zhu YP, Xie C, Jue DW, Hong YB, Chen M, Hubdar AK, Yang Q (2012) Transgenic potato plants expressing StoVe1 exhibit enhanced resistance to Verticillium dahliae. Plant Mol Biol Rep 30: 10321039.

Nembaware V, Seoighe C, Sayed M, Gehring C (2004) A plant natriuretic peptide-like gene in the bacterial pathogen Xanthomonas axonopodis may induce hyper-hydration in the plant host: a hypothesis of molecular mimicry. BMC Evol Biol 4: 10.

Pegg GF (1974) Verticillium diseases. Rev Plant Pathol 53: 157-182.

Pegg GF, Brady BL (2002) Verticillium wilts. Wallingord, Oxfordhire: CABI Publishing.

Schaible L, Cannon OS, Waddoups V (1951) Inheritance of resistance to Verticillium wilt in a tomato cross. Phytopathology 41: 986-990.

Simko I, Costanzo S, Haynes K, Christ B, Jones R (2004) Linkage disequilibrium mapping of a Verticillium dahliae resistance quantitative trait locus in tetraploid potato (Solanum tuberosum) through a candidate gene approach. Theor Appl Genet 108: 217-224.

Simko I, Haynes K, Ewing E, Costanzo S, Christ B, Jones R (2004) Mapping genes for resistance to Verticillium albo-atrum in tetraploid and diploid potato populations using haplotype association tests and genetic linkage analysis. Mol Genet Genomics 271: 522-531.

Thomma BPHJ, Nurnberger T, Joosten MHAJ (2011) Of PAMPs and effectors: The blurred PTI-ETI dichotomy. Plant Cell 23: 4-15.

Vining K, Davis T (2009) Isolation of a $V e$ homolog, $m V e 1$, and its relationship to verticillium wilt resistance in Mentha longifolia (L.) Huds. Mol Genet Genomics 282: 173-184.

Wang G, Fiers M, Ellendorff U, Wang Z, de Wit PJ, Angenent GC, Thomma BPHJ (2010) The diverse roles of extracellular leucine-rich repeat-containing receptor-like proteins in plants. Critl Rev Plant Sci 29: 285-299.

Wang HM, Lin ZX, Zhang XL, Chen W, Guo XP, Nie YC, Li YH (2008) Mapping and quantitative trait loci analysis of verticillium wilt resistance genes in cotton. J Integr Plant Biol 50: 174-182. 


\section{General introduction}

Yang C, Guo W, Li G, Gao F, Lin S, Zhang T (2008) QTLs mapping for Verticillium wilt resistance at seedling and maturity stages in Gossypium barbadense L. Plant Sci 174: 290-298.

Yang Y, Ling X, Chen T, Cai L, Liu T, Wang J, Fan X, Ren Y, Yuan H, Zhu W (2014) A cotton Gbvdr5 gene encoding a leucine-rich repeat receptor-like protein confers resistance to Verticillium dahliae in transgenic Arabidopsis and upland cotton. Plant Mol Biology Rep 33: 1-15.

Zhang B, Yang Y, Chen T, Yu W, Liu T, Li H, Fan X, Ren Y, Shen D, Liu L (2012) Island cotton Gbve1 gene encoding a receptor-like protein confers resistance to both defoliating and non-defoliating isolates of Verticillium dahliae PLOS ONE 7: e51091.

Zhang Y, Wang X, Yang S, Chi J, Zhang G, Ma Z (2011) Cloning and characterization of a Verticillium wilt resistance gene from Gossypium barbadense and functional analysis in Arabidopsis thaliana. Plant Cell Rep 30: 2085-2096.

Zhang Z, Song Y, Liu CM, Thomma BPHJ (2014) Mutational analysis of the Ve1 immune receptor that mediates Verticillium resistance in tomato. PLOS ONE 9: e99511. 



\section{Chapter 2}

\section{Biological functions of effectors secreted by soil-borne plant pathogens}

Yin Song

Laboratory of Phytopathology, Wageningen University, Droevendaalsesteeg 1, 6708 PB Wageningen, the Netherlands 


\section{Chapter 2}

\section{ABSTRACT}

Soil-borne phytopathogenic microbes live in complex and sophisticated belowground environments. To establish themselves within their host plants, these pathogens have to overcome plant defense mechanisms, ranging from preformed barriers to activated defenses. To this end, they typically secrete effector molecules that play important roles to support host colonization through a wide range of mechanisms. Whereas most well-studied effectors are proteinaceous molecules, non-canonical effectors, such as metabolites and small RNA molecules, have also been described. In addition to targeting plant components, part of the effector catalogue may be required for self-defense against, and targeting of, other microbiome partners, including antagonists and competitors. 


\section{INTRODUCTION}

Soil-borne pathogenic microorganisms cause significant yield and quality losses in crops worldwide. They reside in the soil for brief or extended periods of time, where they survive on plant residues or as resting structures such as melanised mycelium, chlamydospores, oospores, or (micro)sclerotia, until root exudates trigger them to become active and invade the roots of plants (Agrios, 2005). Once inside, they may be confined to the root system, or invade above-ground tissues, or again move outside to infect other parts of the root system or roots from neighbouring plants (Agrios, 2005). Plants that are infected by soil-borne pathogens suffer from seedling damping-off, root rot, root blackening, wilting, stunting or plant death (Agrios, 2005). Importantly, since damage to plants by soil-borne pathogens may result from belowground infection, crop yield and quality losses caused by soil-borne pathogens are often underestimated and sometimes even remain unnoticed (De Coninck et al., 2015).

Soil-borne pathogens are notoriously difficult to control due to their general persistence in the soil for long periods of time through the formation of resilient survival structures (Koike et al., 2003; De Coninck et al., 2015). Soil fumigants that were widely used for controlling soil-borne diseases have nowadays largely been banned due to their extremely hazardous effects on the environment as well as on human health. Since many soil-borne pathogens have a wide range of hosts, crop rotation is often ineffective (Reddy, 2012).

The most important soil-borne microbial pathogens belong to three taxonomic groups: fungi, oomycetes and bacteria. The most prominent soil-borne fungal pathogens comprise Fusarium oxysporum (Michielse and Rep, 2009), Verticillium spp. (Fradin and Thomma, 2006; Klosterman et al., 2009; Klimes et al., 2015) and Rhizoctonia solani (Gonzalez et al., 2011), whereas the most notorious oomycetes include Phytophthora spp. (van West et al., 2003; Kamoun et al., 2015) and Pythium spp. (van West et al., 2003). Collectively, these filamentous pathogens are responsible for diseases in hundreds of plant species. Fewer diseases are caused by soil-borne bacterial pathogens, as there are only few soil-borne bacteria infecting roots, such as Agrobacterium spp. (Otten et al., 2008) and Ralstonia solanacearum (Peeters et al., 2013). In addition, soil-borne microbial pathogens occur in other kingdoms, such as Plasmodiophoromycete protists in the eukaryotic kingdom of 


\section{Chapter 2}

Rhizaria, including Plasmodiophora brassicae that is the causal agent of club-root disease of crucifers (Hwang et al., 2012; Schwelm et al., 2015) and Spongospora subterranea that causes powdery scab on potato (Harrison et al., 1997). Here, we summarize the current knowledge on the biological functions of effectors secreted by soil-borne pathogens.

\section{EFFECTOR DISCOVERY}

Effectors are defined as molecules that are secreted by phytopathogenic microbes in order to manipulate host physiology and support host colonization. Although the vast majority of effector activities and host targets presently still remains unknown, at least a subset is experimentally found to suppress immune responses (de Jonge et al., 2011; Rovenich et al., 2014; Cook et al., 2015). Importantly, this host immunity suppression activity implies that effectors can be used as probes to identify vital components of host immune systems and can be used for effector-informed crop improvement (Vleeshouwers and Oliver, 2014).

Recent advances in next-generation sequencing technologies, genome assembly, gene annotation, and effector identification methods hold promise to fully disclose pathogen effector repertoires through computational prediction (Sperschneider et al., 2015; Gibriel et al., 2016). Many bacterial pathogens directly inject effectors into the host cytoplasm via a specialized secretion machinery, namely the type III secretion (T3S) system (Cambronne and Roy, 2006; Galán and Wolf-Watz, 2006). Nterminal sequence signals direct bacterial effectors to the T3S system and thus machine learning approaches have been developed to predict bacterial T3S effectors from sequenced genomes based on $\mathrm{N}$-terminal protein sequence information (McDermott et al., 2011). In oomycetes, consensus N-terminal amino acid motifs, namely the RxLR (Arg-x-Leu-Arg) motif and the LxLFLAK motif, have been proposed to facilitate host cell translocation (Whisson et al., 2007; Schornack et al., 2010). Thus, these motifs can be queried to identify RxLR and Crinkler (CRN) effectors encoded within oomycete genomes (Tyler et al., 2006; Jiang et al., 2008; Haas et al., 2009).

Prediction of proteinaceous effectors of fungal pathogens is less straightforward, as fungal effectors generally lack significant sequence similarity and consensus motifs (Sperschneider et al., 2015). To date, most characterized fungal effectors are small in size (typically less than 300 amino acids) and rich in cysteine residues (four 
or more) (Stergiopoulos and de Wit, 2009). However, these features cannot strictly be used as criteria to identify effector repertoires, as some well-characterized fungal effectors lack these properties. For example, the effectors Cmu1 and ApB73 from the fungal pathogen Ustilago maydis are much larger in size (Djamei et al., 2011; Stirnberg and Djamei, 2016). Thus, to describe potential effector repertories of individual fungal pathogens only rather universal features, such as their secretion and expression in planta, can be considered (Sperschneider et al., 2015). Recently, the first machine learning tool called "EffectorP" was introduced which is trained to improve the prediction of fungal effector proteins from secretomes based on sequence-derived properties, such as sequence length, molecular weight and protein net charge, as well as cysteine, serine and tryptophan content (Sperschneider et al., 2016). When combined with in planta expression data, "EffectorP" is proving useful for prioritizing putative effector candidates (Sperschneider et al., 2016). Although the computational effector prediction has great potential to identify putative effectors, further experimental studies are required to validate their role in plantpathogen interactions.

\section{EFFECTOR EVOLUTION}

In general, effectors are expected to exhibit accelerated evolution, enabling pathogens to escape or overcome recognition, evade or suppress host immunity and support colonization of specific hosts or adapt to new hosts (Stergiopoulos and de Wit, 2009; Raffaele and Kamoun, 2012; Karasov et al., 2014; Seidl and Thomma, 2014; Dong et al., 2015). Effector genes are often found in dynamic genomic regions that show increased rates of recombination, and possibly also mutation, in the genome (Raffaele and Kamoun, 2012; Karasov et al., 2014; Seidl and Thomma, 2014; Dong et al., 2015). Sometimes these regions even concern separate chromosomes that can be transferred between pathogens. In bacteria, pathogenicity islands are clusters of genes that contain an abundance of genes involved in host associations that are located either on the chromosome or on accessary plasmids. These genomic regions contain sequences associated with flanking repeats or transposons as well as with tRNA genes, which are targets for genetic integration and excision (Hacker et al., 1997; McCann and Guttman, 2008). 


\section{Chapter 2}

In filamentous plant pathogens, effector genes are often embedded within highly variable lineage-specific (LS) genomic regions. For example, comparative analysis of the genomes of Fusarium species revealed that Fusarium pathogens carry LS genomic regions that are found as small conditionally dispensable chromosomes (CDCs) containing effector genes (Ma et al., 2010). Further genomic comparisons between CDCs from legume-infecting $F$. oxysporum strains revealed small conserved genomic regions that contain in planta expressed genes encoding secreted effector proteins (Williams et al., 2016). Similarly, genomic comparisons of multiple strains of $V$. dahliae revealed that all $V$. dahliae strains carry LS genomic regions that are significantly enriched for in planta expressed effector genes (de Jonge et al., 2013; Faino et al., 2015; 2016). Genetic flexibility of such dynamic genomic regions is governed by various mechanisms including recombination and activity of transposable elements (TEs) (Raffaele and Kamoun, 2012; Seidl and Thomma, 2014). Genomic rearrangements induce duplications, deletions and translocations, leading to the gain, or loss of genomic material, while TEs can generate a local genomic environment that facilitates genomic rearrangements (Raffaele and Kamoun, 2012; Seidl and Thomma, 2014). For $V$. dahliae it has been shown that LS regions evolved by genomic rearrangements that are mediated by erroneous double-strand repair, often utilizing TEs as a substrate for repair (de Jonge et al., 2013; Faino et al., 2016). TEs seem to contribute to effector evolution in oomycete genomes as well (Haas et al., 2009; Raffaele et al., 2010). These findings illustrate that soil-borne plant pathogens deploy various mechanisms to establish variable genomic regions that are enriched for effectors. Ultimately, these mechanisms maintain effector catalogues that impact pathogen lifestyle and host colonization.

\section{EFFECTORS WITH APOPLASTIC FUNCTIONS}

The initial contact between host plants and soil-borne pathogens is often established in the apoplast, the extracellular spaces of plant tissues. The apoplast is an environment with a relatively low pH (Felle, 1998) that is hostile for microbes because of various plant-secreted hydrolytic enzymes, including chitinases and glucanases, that affect microbial cell wall integrity and release microbial cell wall fragments (Sánchez-Vallet et al., 2015; Rovenich et al., 2016). These released microbial cell wall fragments may activate host immune receptors, leading to 
production of plant-derived proteases, antimicrobial compounds, and lignins for thickening of the plant cell walls that constitute the next host barrier for the pathogen (Hückelhoven, 2007; Sánchez-Vallet et al., 2015; Rovenich et al., 2016). To overcome these biochemical and physical barriers in the apoplast, soil-borne pathogens require effector molecules.

Extracellular alkalinisation of host tissue is thought to contribute to fungal virulence (Prusky and Yakoby, 2003). To thrive in the acidic apoplast, some fungal pathogens secrete effectors to modulate the extracellular pH (Prusky and Yakoby, 2003). The F. oxysporum genome encodes a peptide that is homologous to the plant peptide hormone RALF (rapid alkalinisation factor), which is known to increase extracellular alkalinisation (Murphy and De Smet, 2014). Genetic analysis revealed that functional Fusarium RALF (F-RALF) is required for efficient host alkalinisation and contributes to the virulence of $F$. oxysporum in tomato plants (Masachis et al., 2016). F-RALF homologues are widely distributed in several bacterial species and various fungi, many of which infect plants, including $V$. dahliae and $V$. alfalfae (Masachis et al., 2016; Thynne et al., 2016), implying that other phytopathogenic microbes may use RALF peptides to efficiently induce extracellular alkalinisation in the host, thereby enabling infection.

To protect hyphae from plant-secreted chitinases, fungal pathogens secrete effectors to prevent cell wall hydrolysis by plant chitinases and interfere with host immune receptor activation (Sánchez-Vallet et al., 2015). Vd2LysM is a chitinbinding LysM-containing effector from $V$. dahliae that protects fungal hyphae from degradation by plant hydrolytic enzymes (Kombrink et al., 2017). Similar protective activity mediated by LysM effectors was found in other fungal pathogens (Kombrink and Thomma, 2013; Sánchez-Vallet et al., 2015). Although the molecular mechanism by which LysM effectors protect hyphae against hydrolysis by plant chitinases presently remains elusive, they may form chitin-dependent oligomeric chains to cover fungal hyphae and prevent access of plant chitinases to the chitin in the fungal cell wall (Sánchez-Vallet et al., 2015). This mechanism was previously demonstrated for the Cladosporium fulvum effector Avr4 and the Pseudocercospora fuligena orthologue PfAvr4 that bind to fungal cell walls through an invertebrate chitinbinding domain in order to protect hyphae from host chitinases (van den Burg et al., 2006; Kohler et al., 2016). Alternatively, Vd2LysM protects hyphae through indirect 


\section{Chapter 2}

or direct inhibition of host chitinase activity. It has been proposed that direct inhibition of plant chitinases is a general strategy for fungal pathogens to protect fungal cell walls (Sánchez-Vallet et al., 2015). Indeed, F. oxysporum f. sp. lycopersici secretes a metalloprotease and a serine protease that synergistically cleave extracellular tomato chitinases, leading to compromised antifungal activity and enhanced disease in tomato (Jashni et al., 2015). Similar activities occur in other fungal pathogens, including $V$. dahliae (Jashni et al., 2015). In addition to protecting the fungal cell walls from hydrolysis by plant chitinases, LysM-containing effectors outcompete host immune receptors for chitin fragment binding to interfere with chitin-triggered immunity. The $C$. fulvum LysM effector extracellular protein 6 (Ecp6) binds to chitin fragments with high specificity through its LysM domains to prevent chitin-triggered immunity (de Jonge et al., 2010; Sánchez-Vallet et al., 2013). Similarly, the Vd2LysM effector deregulates chitin-triggered immunity through chitin fragment binding, and contributes to virulence of $V$. dahliae during infection (de Jonge et al., 2013; Kombrink et al., 2017).

In addition to the ability to prevent hydrolysis by plant chitinases and interfere with chitin-triggered immunity, effectors have been shown to inhibit plant glucanases in order to avoid host immune receptor activation (Rose et al., 2002; Sánchez-Rangel et al., 2012). A glucanase inhibitor protein 1 (GIP1) from Phytophthora sojae was shown to directly inhibit a soybean endoglucanase (EGaseA), thus preventing release of oligosaccharides and activation of the corresponding, yet enigmatic, host immune receptor (Rose et al., 2002).

Plants secrete proteases, such as papain-like cysteine proteases (PLCPs), serine and aspartic proteases into the apoplast (Jashni et al., 2015; Misas-Villamil et al., 2016). Several plant proteases are induced in the presence of pathogens, are able to induce expression of plant defense genes, and degrade effectors secreted by pathogens (Jashni et al., 2015; Misas-Villamil et al., 2016). Thus, plant proteases acting as important components of the plant defense system could be targeted by effectors. The tomato serine protease P69B is inhibited by two serine proteases, EPI1 and EPI10 (extracellular proteinase inhibitor 1 and 10), from Phytophthora infestans (Tian et al., 2004; 2005). Similarly, PLCP C14 of tomato and potato is inhibited by two cystatin-like effectors EPIC1 and EPIC2B from P. infestans (Kaschani et al., 2010). Moreover, the tomato protease C14 is also targeted by another P. infestans effector, 
AVRblb2, which prevents C14 secretion into the apoplast (Bozkurt et al., 2011). The finding that $P$. infestans evolved distinct and structurally divergent protease inhibitors to target the same plant protease indicates that inhibition of that particular plant protease is important for P. infestans infection. An additional example for an important plant protease targeted by different phytopathogens is provided by inhibitors of tomato PLCP Rcr3 (Required for Cladosporium resistance3) found in unrelated pathogens (Rooney et al., 2005; Song et al., 2009; LozanoTorres et al., 2012). The sequence-unrelated effectors EPIC1 and EPIC2B from $P$. infestans (Song et al., 2009), Avr2 from C. fulvum (Rooney et al., 2005), and Gr-VAP1 from the nematode Globodera rostochiensis (Lozano-Torres et al., 2012) all inhibit tomato Rcr3. Both effectors Avr2 and EPIC2B also inhibit Pip1 (Phytophthora inhibited protease 1), which is related to tomato Rcr3 (Tian et al., 2007; van Esse et al., 2008). However, the role of these particular plant proteases in plant defense remains unknown.

The necrosis and ethylene-inducing peptide 1 (NEP1) was originally identified in culture filtrates from F. oxsporum (Bailey, 1995), and NEP1 homologues named as NLPs (NEP1-like proteins) found in many bacteria, fungi, and oomycetes that are generally associated with necrotic activity in dicotyledonous plants through plasma membrane permeabilization and cytolysis of plant cells (Ottmann et al., 2009). With the increasing availability of bacterial, fungal, and oomycete genomes, it has become apparent that NLPs are widely distributed in microorganisms (Gijzen and Nürnberger, 2006; Oome and Van den Ackerveken, 2014), and that the NLP family is significantly expanded in several oomycetes (Gijzen and Nürnberger, 2006; Cabral et al., 2012; Dong et al., 2012; Oome and Van den Ackerveken, 2014). Most identified NLPs not only trigger cell death but also elicit strong immune responses in dicots (Gijzen and Nürnberger, 2006; Qutob et al., 2006). There is accumulating evidence that NLP effectors exhibit functional diversification in various pathogens (Dong et al., 2012; Zhou et al., 2012; Santhanam et al., 2013; Oome and Van den Ackerveken, 2014). Characterization of a set of NLPs in V. dahliae indicated that the expanded $V$. dahliae NLP family displays functional diversification, with differential cytotoxicity among the NLP family members (Zhou et al., 2012; Santhanam et al., 2013). Another observation for functional diversification in the $V$. dahliae NLP family comes from the differential contributions of cytotoxic NLP members NLP1 and NLP2 to virulence on 


\section{Chapter 2}

different host plants. Both NLP1 and NLP2 are required for full virulence of $V$. dahliae strain JR2 on tomato and Arabidopsis, while neither of these two is involved in virulence of $V$. dahliae strain V592 on cotton (Zhou et al., 2012; Santhanam et al., 2013). Moreover, NLP1 is required for virulence of $V$. dahliae strain JR2 on Nicotiana benthamiana, whereas NLP2 is not even expressed during $N$. benthamiana infection (Santhanam et al., 2013). One out of 3 P. infestans NLPs tested causes necrosis (Kanneganti et al., 2006), whereas eight out of 19 P. sojae NLPs examined are able to induce necrosis (Dong et al., 2012).

Cell wall-degrading enzymes (CWDEs) that are capable of degradation of cell wall polysaccharides to break down the physical barrier of the plant cell walls during infection can be considered as effectors. Functional redundancy has complicated investigations into the role of individual CWDEs in virulence (Kubicek et al., 2014). However, the importance of CWDEs acting as virulence factors was demonstrated through disruption of the sucrose nonfermenting 1 gene (SNF1) in F. oxysporum and $V$. dahliae. SNF1 encodes part of a kinase complex regulating the expression of CWDEs and SNF1 mutants show compromised virulence (Ospina-Giraldo et al., 2003; Tzima et al., 2011). Other examples showing a positive correlation between individual CWDEs and virulence include studies on $R$. solanacearum, $F$. oxysporum $\mathrm{f}$. sp. lycopersici, V. dahliae, Phytophthora parasitica and P. sojae (Novo et al., 2006; Wu et al., 2008; Poueymiro and Genin, 2009; Maruthachalam et al., 2011; Ma et al., 2015; Bravo Ruiz et al., 2016). These studies conclude that CWDEs are important for virulence. Genome analyses revealed that V. dahliae, V. albo-atrum and F. oxsporum have an expanded arsenal of particular CWDE families compared with other fungi, including a significant expansion of pectin-degrading enzymes (Klosterman et al., 2011; Zhao et al., 2013). It has been hypothesized that expansion of pectinolytic enzymes in V. dahliae, V. albo-atrum and F. oxsporum may facilitate host colonization (Klosterman et al., 2011; Yadeta and Thomma, 2013; Zhao et al., 2013). Genomic analysis revealed that high numbers of pectin-degrading enzymes were also observed in the genome of the broad host-range fungal pathogen $R$. solani (Hane et al., 2014). Enhanced numbers of pectinolytic enzymes in this pathogen facilitate to break down pectinaceous host barriers, presumably providing also this pathogen the capacity to colonize a broad range of plants. 


\section{EFFECTORS WITH CYTOPLASMIC FUNCTIONS}

Some effectors are translocated across the plant cell membrane into the host cytoplasm, where they target plant components in order to benefit pathogen proliferation in the host.

Plant hormones are key signaling molecules that regulate multiple aspects of plant growth, development, and defense (Kazan and Lyons, 2014). Salicylic acid (SA), jasmonic acid (JA), and ethylene (ET) are considered as primary defense phytohormones that act in response to pathogen infection. SA is important for resistance to biotrophic and hemibiotrophic pathogens while JA and ET are associated with resistance to necrotrophic microbes (Pieterse et al., 2012; Fu and Dong, 2013). Components of SA, JA and ET biosynthesis and signaling pathways are targeted by pathogen effectors to promote infection (Kazan and Lyons, 2014). Effectors VdIsc1 and PsIsc1, which are secreted by $V$. dahliae and P. sojae, respectively, are isochorismatases that hydrolyse the SA precursor isochorismate to interfere with the plant SA metabolism pathway and thus promote infection (Liu et al., 2014). Similarly, the effector PbBSMT of the protist P. brassicae disrupts SA signalling through decreasing the accumulation of SA in $A$. thaliana plants and promote tissue colonization (Ludwig-Muller et al., 2015). These examples indicate that reducing SA accumulation level is an important strategy for pathogens to establish infection. Pathogens also secrete effectors to interfere with phytohormone perception and signaling through mimicking of plant hormones. For example, some strains of the bacterial pathogen Pseudomonas syringae produce phytotoxin coronatine (COR), a structural mimic of the bioactive JA hormone JA-Ile (Geng et al., 2012). COR is perceived by the plant JA receptor COI1, activating JA-dependent responses that, in turn, attenuate SA-dependent responses involved in $P$. syringage resistance (Geng et al., 2012). Notably, COR is more active than the host JA-Ile in triggering the JA pathway, indicating that COR acts as a potent and highly specific mimic of JA-Ile perception in plants (Geng et al., 2012). In fact, bioactive JAs are produced by some F. oxysporum strains (Brodhun et al., 2013; Cole et al., 2014). JAinsensitive Arabidopsis mutants display enhanced resistance against Arabidopsisinfecting F. oxysporum strains that produce detectable JAs (Cole et al., 2014). In contrast, JA-insensitive Arabidopsis and tomato mutants do not show altered resistance against Arabidopsis- and tomato-infecting F. oxsporum strains that do not 


\section{Chapter 2}

produce JAs, respectively (Cole et al., 2014). Thus, some strains of F. oxsporum appear to secrete JAs as effectors, but the mechanism how F. oxsporum-secreted JAs promote infection is unknown.

Reactive oxygen species (ROS), including hydrogen peroxide $\left(\mathrm{H}_{2} \mathrm{O}_{2}\right)$, play an essential role in plant defense against pathogens (Heller and Tudzynski, 2011). Some effectors have been shown to interfere with production of ROS in order to promote virulence. Plant catalases are localized to the peroxisome and convert $\mathrm{H}_{2} \mathrm{O}_{2}$ into $\mathrm{H}_{2} \mathrm{O}$ and $\mathrm{O}_{2}$, thus contributing to ROS homeostasis in plant cells (Mhamdi et al., 2010). Two CRN effectors, PsCRN63 and PsCRN115, from P. sojae interact with plant catalases to regulate plant cell death and $\mathrm{H}_{2} \mathrm{O}_{2}$ homeostasis to promote infection (Zhang et al., 2015). PsCRN63 promotes plant cell death through interacting with and destabilizing plant catalases, thus increasing $\mathrm{H}_{2} \mathrm{O}_{2}$ accumulation. PsCRN115, however, counteracts these processes to decrease $\mathrm{H}_{2} \mathrm{O}_{2}$ levels and inhibit plant cell death (Zhang et al., 2015). Another CRN effector, PsCRN70 from P. sojae, suppresses plant cell death, $\mathrm{H}_{2} \mathrm{O}_{2}$ accumulation and expression of defense-related genes, to enhance pathogen infection (Rajput et al., 2014). However, the mechanism how PsCRN70 regulates plant ROS production remains unknown. Recently, the type III effector RipAK from $R$. solanacearum has also been shown to interact with and inhibit plant catalases and suppresses plant immunity, thus resulting in enhanced bacterial infection (Sun et al., 2017). These findings clearly show that modulation of plant ROS production by pathogens through effectors is important for disease development.

The plant ubiquitin-proteasome system plays a central role in many cellular processes including defense responses and hormone signaling, and thus is an essential target of effectors (Banfield, 2015). GALA proteins (also named RipG [Ralstonia injected proteins $G$ proteins]) are secreted by the T3S system of $R$. solanacearum, which contain a conserved GAxALA amino acid motif in the C-terminal leucine-rich repeat region and a plant-type F-box domain in the N-terminal region (Cunnac et al., 2004). The GALA effector is able to interact with the $A$. thaliana SKP1like protein (ASK), a component of the plant SCF ( ubiquitin ligase, suggesting that the GALA effector may interfere with the ubiquitinproteasome pathway and promote infection (Angot et al., 2006). GALA7 was shown to be a host-specificity factor required for disease promotion on Medicago truncatula plants and its F-box domain was found to be essential for its virulence function 
(Angot et al., 2006). Mutants lacking all seven GALA genes in $R$. solanacearum strains GMI1000 display compromised virulence on diverse plants (Angot et al., 2006; Remigi et al., 2011). However, it is yet unknown whether GALAs contribute to degradation of host targets. Similar to GALAs, the F-box domain containing VirF, which is secreted by the type IV secretion system of $A$. tumefaciens, was previously shown to interact with $A$. thaliana ASKs and promote virulence during $A$. tumefaciens infection (Schrammeijer et al., 2001). VirF interacts with plant VIP1 (VirE2interacting protein 1) and targets VIP1 into the host ubiquitin-proteasome for proteolysis (Tzfira et al., 2004). In P. infestans, the RxLR effector Avr3a was shown to interact with and stabilize the host ubiquitin E3 ligase CMPG1 for suppression of INF1-induced host cell death during biotrophic growth (González-Lamothe et al., 2006; Bos et al., 2010). These examples illustrate that the host ubiquitin-proteasome system can be exploited by pathogens through effectors for their own benefit.

Plant perception of microbial pathogens leads to transcriptional reprogramming towards host defense against pathogens. In response, pathogens secrete effectors to reprogram host gene expression by targeting defense-related transcription factors, activating the expression of host susceptibility genes and interfering with host post-transcriptional gene silencing in order to establish infection (Toruño et al., 2016). A number of host transcription factors that regulate the expression of the host defense-related genes appear to be targets of effectors. Effectors can directly target host transcription factors in order to downregulate host genes involved in defense responses. Plant WRKY transcription factors, known for the canonical WRKYGQK DNA-binding motif, are key components that regulate host defense responses against several pathogens (Rushton et al., 2010; Chi et al., 2013). The acetyltransferase PopP2 (RipP2) from R. solanacearum localizes to the plant cell nucleus and directly acetylates lysine residues in the WRKYGQK DNA-binding motif of WRKY transcription factors (Deslandes et al., 2003; Le Roux et al., 2015; Sarris et al., 2015). Acetylation by PopP2 interferes with the DNA binding of WRKY transcription factors and thus with the transcriptional activation of WRKY transcription factor-regulated immune genes, leading to enhanced virulence (Le Roux et al., 2015; Sarris et al., 2015). In addition to WRKY transcription factors, PopP2 interacts with and acetylates two lysine residues in the C-terminal WRKYGQK domain of the $A$. thaliana intracellular immune receptor RRS1-R ( 


\section{Chapter 2}

Ralstonia solanacearum 1). Acetylation of RRS1-R by PopP2 disrupts the binding of RRS1-R to W-box DNA sequences, leading to the activation of the RRS1-R-associated

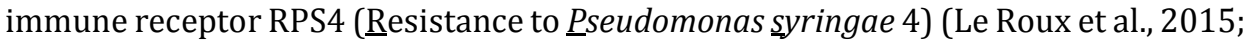
Sarris et al., 2015). P. infestans secretes the RxLR effector Pi03192 that interacts with two plant NAC transcription factors NTP1 and NTP2 (NAC Targeted by $\underline{\text { Phytophthora }}$ 1 and 2) at the endoplasmic reticulum (ER) membrane and prevents their localization from ER to the nucleus to increase host susceptibility (McLellan et al., 2013). Finally, the Phytophthora capsici CRN effector CRN12-997 was shown to directly bind to the tomato immune-related transcription factor SITCP14-2, resulting in compromised immunity mediated by SITCP14-2 (Stam et al., 2013). CRN12-997 affects SITCP14-2 association with nuclear chromatin and SITCP14-2 sub-nuclear localization, preventing SITCP14-2 from positively regulating defense against $P$. capsici (Stam et al., 2013). These examples illustrate show how effectors prevent the activation of host defense through modification of transcription factor DNA binding site or mislocalization of transcription factors during host colonization.

Pathogen effectors can directly act as plant transcription factors and induce the expression of host susceptibility genes. Ralstonia transcription activator-like effectors (TALEs), termed RipTALEs that are found in many $R$. solanacearum strains are structurally homologous to Xanthomonas TALEs (Lange et al., 2013; Li et al., 2013). Xanthomonas TALEs are injected into host cells and enable to directly activate host target genes through a repeat structure domain involved in the binding to specific DNA sequences, known as effector binding elements (EBEs), at promoters of host target genes (Boch and Bonas, 2010). Among the plant genes targeted by TALEs are those encoding proteins involved in development, stress responses and sugar transport. Rice SWEET genes are involved in sugar transport and activated by TALEs from the rice bacterial pathogen Xanthomonas oryzae pv. oryzae, presumably facilitating sugar export for bacterial consumption and promoting infection (Boch et al., 2014; Chen, 2014). Characterization of Xanthomonas TALE DNA-binding specificity has uncovered several examples of host target genes that confer bacterial susceptibility as well as target genes present in resistant plants that induce disease resistance (Boch et al., 2014). It was shown that RipTALEs can also act as transcription activators in plant cells (Lange et al., 2013; Li et al., 2013). However, host target genes of RipTAL effectors remain unknown. 
RNA silencing (or post-transcriptional gene silencing) serves as a major defense mechanism against viruses in plants. To counter host antiviral responses, viruses employ suppressors of RNA silencing that interfere with the host RNA silencing machinery and favour viruses to proliferate within the host (Vance and Vaucheret, 2001). However, plant viruses are not the only microorganisms to interfere with the plant RNA silencing machinery. P. sojae delivers two RxLR effectors, PSR1 and PSR2 (Phytophthora suppressor of RNA silencing 1 and 2), that act as suppressors of plant RNA silencing and enhance susceptibility to P. sojae (Qiao et al., 2013). Further, PSR1 was shown to interact with an evolutionarily conserved nuclear protein known as the PSR1-interacting protein 1 (PINP1) which regulates accumulation of small RNAs. Thus, PSR1 affects the biogenesis of plant small RNAs to promote infection (Qiao et al., 2015). Fungal pathogens can also manipulate RNA silencing machinery. V. dahliae secretes small RNAs that target the Arabidopsis RNA silencing component Argonaute (AG01), downregulate the expression of host defense genes and thus promote fungal infection (Wang et al., 2016). A similar mechanism was previously demonstrated for the fungal pathogen Botrytis cinerea-derived small RNAs target the Arabidopsis AG01 to impair the expression of host defense genes for promoting fungal virulence (Weiberg et al., 2013). These examples illustrate that the host RNA silencing machinery can be exploited by soil-borne pathogens through effectors to establish infection.

\section{EFFECTORS PLAY ROLES IN SELF-DEFENSE AND COMPETITION}

Before establishing themselves within a host plant, soil-borne pathogens need to interact with numerous microbial competitors, antagonists, or hyperparasites within the microbe-rich belowground environment. Likely, antagonists and hyperparasites produce a range of antimicrobial compounds and lytic enzymes to attack soil-borne pathogens. Consequently, soil-borne pathogens require molecules for self-defense and interaction with other microbes. For example, the phenolic antibiotic 2,4diacetylphloroglucinol (DAPG) from Gram-negative antagonistic bacteria is toxic towards a wide range of pathogenic microbes, including fungal plant pathogens (Raaijmakers et al., 2009). It was shown that DAPG tolerance is positively correlated with the ability of $F$. oxysporum strains to degrade this antimicrobial metabolite via deacetylation into the less fungitoxic derivatives monoacetylphloroglucinol and 


\section{Chapter 2}

phloroglucinol (Schouten et al., 2004). This finding suggests that degradation of antimicrobial compounds produced by antagonistic microorganisms is an important self-protection strategy for DAPG-tolerant $F$. oxysporum strains, although the exact molecules secreted by tolerant $F$. oxysporum isolates that metabolizes DAPG remain to be identified. Moreover, F. oxysporum produces the secondary metabolite fusaric acid that appears to specifically repress the expression of DAPG biosynthetic genes in the antagonistic bacterium Pseudomonas fluorescens CHA0 through increasing the binding characteristics of the bacterial repressor-promotor complex (Schnider-Keel et al., 2000; Notz et al., 2002). In addition to self-defense, effectors secreted by soil-borne pathogens act in interaction with microbial competitors. A. tumefaciens uses the type VI secretion (T6S) system to secrete a DNase effector Tde that exhibits a potent antibacterial DNase activity and confer a competitive advantage to A. tumefaciens during host colonization (Ma et al., 2014).The toxic activity of the Tde DNase is counteracted by a cognate immunity protein, termed Tdi, protecting the toxin DNase-producing bacterium from selfkilling. The $A$. tumefacines uses Tde to attack both intra- and inter-species bacterial competitors inside a plant host (Ma et al., 2014). The Tde and Tdi couples are broadly conserved among Gram-negative bacteria, suggesting a widespread antibacterial weapon beneficial for niche colonization (Ma et al., 2014).

\section{EFFECTOR RECOGNITION}

Plant immunity has been described as a multi-layered recognition system to prevent microbial infections (Jones and Dangl, 2006). The first layer involves the perception of microbe-associated molecular patterns (MAMPs), which are conserved across classes of microbes, by cell surface-localized pattern recognition receptors (PRRs) (Jones and Dangl, 2006). Activation of PRRs leads to MAMPs-triggered immunity (MTI) and acts as an early warning system against a wide range of potential pathogens (Jones and Dangl, 2006). Adapted pathogens are able to subvert these early defense responses by escaping or suppressing PTI through the activity of secreted effector molecules, resulting in effector-triggered susceptibility (Jones and Dangl, 2006). In turn, particular plant genotypes have evolved resistance (R) proteins that recognize particular effectors and activate effector-triggered immunity (ETI), which is recognized as a second layer of plant innate immunity (Jones and 
Dangl, 2006). Although described as separate layers of defense, numerous studies on various plant-microbe interactions have revealed that the delineation between MTI and ETI is not strict, but rather a continuum (Thomma et al., 2011). For example, tomato Ve1 recognizes the effector protein Ave1 that is secreted by race 1 strains of Verticillium spp. and contributes to fungal virulence on susceptible plant genotypes, leading to race-specific Verticillium resistance in tomato (Kawchuk et al., 2001; Fradin et al., 2009; de Jonge et al., 2012). Interestingly, homologues of Ave1 were found in plants but also in a number of plant pathogenic microbes. Ave1 is recognized not only by tomato Ve1, but also by a homologue from Nicotiana glutinosa (Zhang et al., 2013). Furthermore, Ve1 also mediates resistance against F. oxysporum f. sp. lycopersici in tomato (de Jonge et al., 2012), demonstrating involvement of this racespecific Ve1 protein in resistance against multiple fungal pathogens. Heterologous expression of tomato Ve1 in Arabidopsis leads to race-specific Verticillium resistance triggered by the effector Ave1 (Fradin et al., 2011; de Jonge et al., 2012). These findings, combined with the observation that Ve1 mediates recognition of Ave1 homologues from multiple plant pathogens (de Jonge et al., 2012), imply that Ve1 has traits of a typical race-specific $R$ protein as well as of a typical PRR, and indicate that $\mathrm{R}$ proteins and PRRs exist on a continuum in plant innate immunity (Fradin et al., 2011; Thomma et al., 2011).

NLPs act as effectors that positively contribute to pathogen virulence (Gijzen and Nürnberger, 2006; Ottmann et al., 2009), indicating that NLP effector-triggered immunity could be considered as belonging to ETI. On the other hand, the widespread distribution of NLPs among diverse microbes and perception of the conserved motif, nlp20 or nlp24, by a typical cell surface-localized complex (RLP23SOBIR1-BAK1 complex) (Böhm et al., 2014; Oome et al., 2014; Albert et al., 2015) appears as a feature of pattern recognition in PTI. Thus, also plant detection of NLP effectors blurs the PTI-ETI dichotomy and illustrates a continuum between PTI-ETI. These examples, and the conceptual conflict that MAMPs are defined from the perspective of the host whereas effectors are defined from the perspective of the invader, recently inspired the proposal of the Invasion Model, in which host immune receptors (termed invasion pattern receptors; IPRs) detect either an externally encoded or modified-self ligand that betrays invaders (termed invasion patterns; IPs) (Cook et al., 2015). This model recognizes that ultimately any immune receptor can 


\section{Chapter 2}

be effective as long as it accurately betrays pathogen presence and elicits an appropriate response, irrespective of whether it recognizes a pathogen- or hostderived ligand that is either conserved or lineage-specific.

\section{ACKNOWLEDGEMENTS}

Y.S. acknowledges a PhD fellowship from the China Scholarship Council (CSC). 


\section{REFERENCES}

Agrios G (2005) Plant Pathology, $5^{\text {th }}$ edn. Elsevier Academic Press, New York.

Albert I, Böhm H, Albert M, Feiler CE, Imkampe J, Wallmeroth N, Brancato C, Raaymakers TM, Oome S, Zhang H, Krol E, Grefen C, Gust AA, Chai J, Hedrich R, Van den Ackerveken G, Nürnberger T (2015) An RLP23-SOBIR1-BAK1 complex mediates NLP-triggered immunity. Nat Plants 1: 15140.

Angot A, Peeters N, Lechner E, Vailleau F, Baud C, Gentzbittel L, Sartorel E, Genschik P, Boucher C, Genin S (2006) Ralstonia solanacearum requires F-box-like domain-containing type III effectors to promote disease on several host plants. Proc Natl Acad Sci USA 103: 14620-14625.

Bailey BA (1995) Purification of a protein from culture filtrates of Fusarium oxysporum that induces ethylene and necrosis in leaves of Erythroxylum coca. Phytopathology 85: 1250-1255.

Banfield MJ (2015) Perturbation of host ubiquitin systems by plant pathogen/pest effector proteins. Cell Microbiol 17: 18-25.

Boch J, Bonas U (2010) Xanthomonas AvrBs3 family-type III effectors: discovery and function. Annu Rev Phytopathol 48: 419-436.

Boch J, Bonas U, Lahaye T (2014) TAL effectors-pathogen strategies and plant resistance engineering. New Phytol 204: 823-832.

Böhm H, Albert I, Oome S, Raaymakers TM, Van den Ackerveken G, Nürnberger T (2014) A aonserved peptide pattern from a widespread microbial virulence factor triggers pattern-induced immunity in Arabidopsis. PLOS Pathog 10: e1004491.

Bos JI, Armstrong MR, Gilroy EM, Boevink PC, Hein I, Taylor RM, Zhendong T, Engelhardt S, Vetukuri RR, Harrower B (2010) Phytophthora infestans effector AVR3a is essential for virulence and manipulates plant immunity by stabilizing host E3 ligase CMPG1. Proc Natl Acad Sci USA 107: 99099914.

Bozkurt TO, Schornack S, Win J, Shindo T, Ilyas M, Oliva R, Cano LM, Jones AM, Huitema E, Van der Hoorn RA (2011) Phytophthora infestans effector AVRblb2 prevents secretion of a plant immune protease at the haustorial interface. Proc Natl Acad Sci USA 108: 20832-20837.

Bravo Ruiz G, Di Pietro A, Roncero MIG (2016) Combined action of the major secreted exo-and endopolygalacturonases is required for full virulence of Fusarium oxysporum. Mol Plant Pathol 17: 339-353.

Brodhun F, Cristobal-Sarramian A, Zabel S, Newie J, Hamberg M, Feussner I (2013) An iron 13Slipoxygenase with an $\alpha$-linolenic acid specific hydroperoxidase activity from Fusarium oxysporum. PLOS ONE 8: e64919.

Cabral A, Oome S, Sander N, Küfner I, Nürnberger T, Van den Ackerveken G (2012) Nontoxic Nep1like proteins of the downy mildew pathogen Hyaloperonospora arabidopsidis: repression of necrosis-inducing activity by a surface-exposed region. Mol Plant-Microbe Interact 25: 697-708.

Cambronne ED, Roy CR (2006) Recognition and delivery of effector proteins into eukaryotic cells by bacterial secretion systems. Traffic 7: 929-939.

Chen LQ (2014) SWEET sugar transporters for phloem transport and pathogen nutrition. New Phytol 201: 1150-1155.

Chi Y, Yang Y, Zhou Y, Zhou J, Fan B, Yu J-Q, Chen Z (2013) Protein-protein interactions in the regulation of WRKY transcription factors. Mol Plant 6: 287-300.

Cole SJ, Yoon AJ, Faull KF, Diener AC (2014) Host perception of jasmonates promotes infection by Fusarium oxysporum formae speciales that produce isoleucine-and leucine-conjugated jasmonates. Mol Plant Pathol 15: 589-600. 


\section{Chapter 2}

Cook DE, Mesarich CH, Thomma BPHJ (2015) Understanding plant immunity as a surveillance system to detect invasion. Annu Rev Phytopathol 53: 541-563.

Cunnac S, Occhialini A, Barberis P, Boucher C, Genin S (2004) Inventory and functional analysis of the large Hrp regulon in Ralstonia solanacearum: identification of novel effector proteins translocated to plant host cells through the type III secretion system. Mol Microbiol 53: 115-128.

De Coninck B, Timmermans P, Vos C, Cammue BP, Kazan K (2015) What lies beneath: belowground defense strategies in plants. Trends Plant Sci 20: 91-101.

de Jonge R, Bolton MD, Kombrink A, van den Berg GC, Yadeta KA, Thomma BPHJ (2013) Extensive chromosomal reshuffling drives evolution of virulence in an asexual pathogen. Genome Res 23: 1271-1282.

de Jonge R, Bolton MD, Thomma BPHJ (2011) How filamentous pathogens co-opt plants: the ins and outs of fungal effectors. Curr Opin Plant Biol 14: 400-406.

de Jonge R, van Esse HP, Kombrink A, Shinya T, Desaki Y, Bours R, van der Krol S, Shibuya N, Joosten MH, Thomma BPHJ (2010) Conserved fungal LysM effector Ecp6 prevents chitin-triggered immunity in plants. Science 329: 953-955.

de Jonge R, van Esse HP, Maruthachalam K, Bolton MD, Santhanam P, Saber MK, Zhang Z, Usami T, Lievens B, Subbarao KV, Thomma BPHJ (2012) Tomato immune receptor Ve1 recognizes effector of multiple fungal pathogens uncovered by genome and RNA sequencing. Proc Natl Acad Sci USA 109: $5110-5115$.

Deslandes L, Olivier J, Peeters N, Feng DX, Khounlotham M, Boucher C, Somssich I, Genin S, Marco Y (2003) Physical interaction between RRS1-R, a protein conferring resistance to bacterial wilt, and PopP2, a type III effector targeted to the plant nucleus. Proc Natl Acad Sci USA 100: 8024-8029.

Djamei A, Schipper K, Rabe F, Ghosh A, Vincon V, Kahnt J, Osorio S, Tohge T, Fernie AR, Feussner I (2011) Metabolic priming by a secreted fungal effector. Nature 478: 395-398.

Dong S, Kong G, Qutob D, Yu X, Tang J, Kang J, Dai T, Wang H, Gijzen M, Wang Y (2012) The NLP toxin family in Phytophthora sojae includes rapidly evolving groups that lack necrosis-inducing activity. Mol Plant-Microbe Interact 25: 896-909.

Dong S, Raffaele S, Kamoun S (2015) The two-speed genomes of filamentous pathogens: waltz with plants. Curr Opin Genet Dev 35: 57-65.

Faino L, Seidl MF, Datema E, van den Berg GC, Janssen A, Wittenberg AH, Thomma BPHJ (2015) Single-molecule real-time sequencing combined with optical mapping yields completely finished fungal genome. mBio 6: e00936-00915.

Faino L, Seidl MF, Shi-Kunne X, Pauper M, van den Berg GC, Wittenberg AH, Thomma BPHJ (2016) Transposons passively and actively contribute to evolution of the two-speed genome of a fungal pathogen. Genome Res 26: 1091-1100.

Felle HH (1998) The apoplastic pH of the Zea mays root cortex as measured with pH-sensitive microelectrodes: aspects of regulation. J Exp Bot 49: 987-995.

Fradin EF, Abd-El-Haliem A, Masini L, van den Berg GC, Joosten MH, Thomma BPHJ (2011) Interfamily transfer of tomato Ve1 mediates Verticillium resistance in Arabidopsis. Plant Physiol 156: 2255-2265.

Fradin EF, Thomma BPHJ (2006) Physiology and molecular aspects of Verticillium wilt diseases caused by V. dahliae and V. albo-atrum. Mol Plant Pathol 7: 71-86.

Fradin EF, Zhang Z, Ayala JCJ, Castroverde CD, Nazar RN, Robb J, Liu C-M, Thomma BPHJ (2009) Genetic dissection of Verticillium wilt resistance mediated by tomato Ve1. Plant Physiol 150: 320332. 
Fu ZQ, Dong X (2013) Systemic acquired resistance: turning local infection into global defense. Annu Rev Plant Biol 64: 839-863.

Galán JE, Wolf-Watz H (2006) Protein delivery into eukaryotic cells by type III secretion machines. Nature 444: 567-573.

Geng X, Cheng J, Gangadharan A, Mackey D (2012) The coronatine toxin of Pseudomonas syringae is a multifunctional suppressor of Arabidopsis defense. Plant Cell 24: 4763-4774.

Gibriel HA, Thomma BPHJ, Seidl MF (2016) The age of effectors: Genome-based discovery and applications. Phytopathology 106: 1206-1212.

Gijzen M, Nürnberger $\mathbf{T}$ (2006) Nep1-like proteins from plant pathogens: recruitment and diversification of the NPP1 domain across taxa. Phytochemistry 67: 1800-1807.

González-Lamothe R, Tsitsigiannis DI, Ludwig AA, Panicot M, Shirasu K, Jones JD (2006) The U-box protein CMPG1 is required for efficient activation of defense mechanisms triggered by multiple resistance genes in tobacco and tomato. Plant Cell 18: 1067-1083.

Gonzalez M, Pujol M, Metraux JP, Gonzalez-Garcia V, Bolton MD, Borrás-Hidalgo 0 (2011) Tobacco leaf spot and root rot caused by Rhizoctonia solani Kühn. Mol Plant Pathol 12: 209-216.

Haas BJ, Kamoun S, Zody MC, Jiang RH, Handsaker RE, Cano LM, Grabherr M, Kodira CD, Raffaele S, Torto-Alalibo T (2009) Genome sequence and analysis of the Irish potato famine pathogen Phytophthora infestans. Nature 461: 393-398.

Hacker J, Blum-Oehler G, Mühldorfer I, Tschäpe H (1997) Pathogenicity islands of virulent bacteria: structure, function and impact on microbial evolution. Mol Microbiol 23: 1089-1097.

Hane JK, Anderson JP, Williams AH, Sperschneider J, Singh KB (2014) Genome sequencing and comparative genomics of the broad host-range pathogen Rhizoctonia solani AG8. PLOS Genet 10: e1004281.

Harrison J, Searle R, Williams N (1997) Powdery scab disease of potato-a review. Plant Pathol 46: 125.

Heller J, Tudzynski P (2011) Reactive oxygen species in phytopathogenic fungi: signaling, development, and disease. Annu Rev Phytopathol 49: 369-390.

Hückelhoven R (2007) Cell wall-associated mechanisms of disease resistance and susceptibility. Annu Rev Phytopathol 45: 101-127.

Hwang SF, Strelkov SE, Feng J, Gossen BD, Howard RJ (2012) Plasmodiophora brassicae: a review of an emerging pathogen of the Canadian canola (Brassica napus) crop. Mol Plant Pathol 13: 105-113.

Jashni MK, Dols IH, Iida Y, Boeren S, Beenen HG, Mehrabi R, Collemare J, de Wit PJM (2015) Synergistic action of a metalloprotease and a serine protease from Fusarium oxysporum f. sp. lycopersici cleaves chitin-binding tomato chitinases, reduces their antifungal activity, and enhances fungal virulence. Mol Plant-Microbe Interact 28: 996-1008.

Jashni MK, Mehrabi R, Collemare J, Mesarich CH, de Wit PJM (2015) The battle in the apoplast: further insights into the roles of proteases and their inhibitors in plant-pathogen interactions. Front Plant Sci 6: 584.

Jiang RH, Tripathy S, Govers F, Tyler BM (2008) RXLR effector reservoir in two Phytophthora species is dominated by a single rapidly evolving superfamily with more than 700 members. Proc Natl Acad Sci USA 105: 4874-4879.

Jones JD, Dangl JL (2006) The plant immune system. Nature 444: 323-329.

Kamoun S, Furzer O, Jones JD, Judelson HS, Ali GS, Dalio RJ, Roy SG, Schena L, Zambounis A, Panabières F (2015) The top 10 oomycete pathogens in molecular plant pathology. Mol Plant Pathol 16: 413-424. 
Kanneganti T-D, Huitema E, Cakir C, Kamoun S (2006) Synergistic interactions of the plant cell death pathways induced by Phytophthora infestans Nep1-like protein PiNPP1.1 and INF1 elicitin. Mol Plant-Microbe Interact 19: 854-863.

Karasov TL, Horton MW, Bergelson J (2014) Genomic variability as a driver of plant-pathogen coevolution? Curr Opin Plant Biol 18: 24-30.

Kaschani F, Shabab M, Bozkurt T, Shindo T, Schornack S, Gu C, Ilyas M, Win J, Kamoun S, van der Hoorn RA (2010) An effector-targeted protease contributes to defense against Phytophthora infestans and is under diversifying selection in natural hosts. Plant Physiol 154: 1794-1804.

Kawchuk LM, Hachey J, Lynch DR, Kulcsar F, van Rooijen G, Waterer DR, Robertson A, Kokko E, Byers R, Howard RJ (2001) Tomato Ve disease resistance genes encode cell surface-like receptors. Proc Natl Acad Sci USA 98: 6511-6515.

Kazan K, Lyons R (2014) Intervention of phytohormone pathways by pathogen effectors. Plant Cell 26: 2285-2309.

Klimes A, Dobinson KF, Klosterman SJ, Thomma BPHJ (2015) Genomics spurs rapid advances in our understanding of the basic biology of vascular wilt pathogens in the genus Verticillium. Annu Rev Phytopathol 53: 181-198.

Klosterman SJ, Atallah ZK, Vallad GE, Subbarao KV (2009) Diversity, pathogenicity, and management of Verticillium species. Annu Rev Phytopathol 47: 39-62.

Klosterman SJ, Subbarao KV, Kang S, Veronese P, Gold SE, Thomma BPHJ, Chen Z, Henrissat B, Lee Y-H, Park J (2011) Comparative genomics yields insights into niche adaptation of plant vascular wilt pathogens. PLOS Pathog 7: e1002137.

Kohler AC, Chen L-H, Hurlburt N, Salvucci A, Schwessinger B, Fisher AJ, Stergiopoulos I (2016) Structural analysis of an Avr4 effector ortholog offers insight into chitin-binding and recognition by the Cf-4 receptor. Plant Cell DOI: https://doi.org/10.1105/tpc.15.00893.

Koike S, Subbarao K, Davis RM, Turini T (2003) Vegetable diseases caused by soilborne pathogens. California, CA: University of California; ANR Publication.

Kombrink A, Rovenich H, Shi-Kunne X, Rojas-Padilla E, van den Berg G, Domazakis E, De Jonge R, Valkenburg DJ, Sánchez-Vallet A, Seidl MF, Thomma BPHJ (2017) Verticillium dahliae LysM effectors differentially contribute to virulence on plant hosts. Mol Plant Pathol 18: 596-608.

Kombrink A, Thomma BPHJ (2013) LysM effectors: secreted proteins supporting fungal life. PLOS Pathog 9: e1003769.

Kubicek CP, Starr TL, Glass NL (2014) Plant cell wall-degrading enzymes and their secretion in plantpathogenic fungi. Annu Rev Phytopathol 52: 427-451.

Lange 0, Schreiber T, Schandry N, Radeck J, Braun KH, Koszinowski J, Heuer H, Strauß A, Lahaye T (2013) Breaking the DNA-binding code of Ralstonia solanacearum TAL effectors provides new possibilities to generate plant resistance genes against bacterial wilt disease. New Phytol 199: 773786.

Le Roux C, Huet G, Jauneau A, Camborde L, Tremousaygue D, Kraut A, Zhou B, Levaillant M, Adachi H, Yoshioka H, Raffaele S, Berthome R, Coute Y, Parker JE, Deslandes L (2015) A receptor pair with an integrated decoy converts pathogen disabling of transcription factors to immunity. Cell 161: 1074-1088.

Li L, Atef A, Piatek A, Ali Z, Piatek M, Aouida M, Sharakuu A, Mahjoub A, Wang G, Khan S (2013) Characterization and DNA-binding specificities of Ralstonia TAL-like effectors. Mol Plant 6: 13181330. 
Liu T, Song T, Zhang X, Yuan H, Su L, Li W, Xu J, Liu S, Chen L, Chen T, Gu L, Zhang B, Dou D (2014) Unconventionally secreted effectors of two filamentous pathogens target plant salicylate biosynthesis. Nat Commun 5: 4686.

Lozano-Torres JL, Wilbers RH, Gawronski P, Boshoven JC, Finkers-Tomczak A, Cordewener JH, America AH, Overmars HA, Van't Klooster JW, Baranowski L (2012) Dual disease resistance mediated by the immune receptor Cf- 2 in tomato requires a common virulence target of a fungus and a nematode. Proc Natl Acad Sci USA 109: 10119-10124.

Ludwig-Muller J, Julke S, Geiss K, Richter F, Mithofer A, Sola I, Rusak G, Keenan S, Bulman S (2015) A novel methyltransferase from the intracellular pathogen Plasmodiophora brassicae methylates salicylic acid. Mol Plant Pathol 16: 349-364.

Ma LJ, van der Does HC, Borkovich KA, Coleman JJ, Daboussi MJ, Di Pietro A, Dufresne M, Freitag M, Grabherr M, Henrissat B, Houterman PM, Kang S, Shim WB, Woloshuk C, Xie X, Xu JR, Antoniw J, Baker SE, Bluhm BH, Breakspear A, Brown DW, Butchko RA, Chapman S, Coulson R, Coutinho PM, Danchin EG, Diener A, Gale LR, Gardiner DM, Goff S, Hammond-Kosack KE, Hilburn K, Hua-Van A, Jonkers W, Kazan K, Kodira CD, Koehrsen M, Kumar L, Lee YH, Li L, Manners JM, Miranda-Saavedra D, Mukherjee M, Park G, Park J, Park SY, Proctor RH, Regev A, Ruiz-Roldan MC, Sain D, Sakthikumar S, Sykes S, Schwartz DC, Turgeon BG, Wapinski I, Yoder O, Young S, Zeng Q, Zhou S, Galagan J, Cuomo CA, Kistler HC, Rep M (2010) Comparative genomics reveals mobile pathogenicity chromosomes in Fusarium. Nature 464: 367-373.

Ma LS, Hachani A, Lin JS, Filloux A, Lai EM (2014) Agrobacterium tumefaciens deploys a superfamily of type VI secretion DNase effectors as weapons for interbacterial competition in planta. Cell Host Microbe 16: 94-104.

Ma Z, Song T, Zhu L, Ye W, Wang Y, Shao Y, Dong S, Zhang Z, Dou D, Zheng X, Tyler BM, Wang Y (2015) A Phytophthora sojae glycoside hydrolase 12 protein is a major virulence factor during soybean infection and is recognized as a PAMP. Plant cell 27: 2057-2072.

Maruthachalam K, Klosterman S, Kang S, Hayes R, Subbarao K (2011) Identification of pathogenicityrelated genes in the vascular wilt fungus Verticillium dahliae by Agrobacterium tumefaciensmediated T-DNA insertional mutagenesis. Mol Biotechnol 49: 209-221.

Masachis S, Segorbe D, Turrà D, Leon-Ruiz M, Fürst U, El Ghalid M, Leonard G, Richards TA, Felix G, Di Pietro A (2016) A fungal pathogen secretes plant alkalinizing peptides to increase infection. Nat Microbiol 1: 16043.

McCann HC, Guttman DS (2008) Evolution of the type III secretion system and its effectors in plantmicrobe interactions. New Phytol 177: 33-47.

McDermott JE, Corrigan A, Peterson E, Oehmen C, Niemann G, Cambronne ED, Sharp D, Adkins JN, Samudrala R, Heffron F (2011) Computational prediction of type III and IV secreted effectors in gram-negative bacteria. Infect Immun 79: 23-32.

McLellan H, Boevink PC, Armstrong MR, Pritchard L, Gomez S, Morales J, Whisson SC, Beynon JL, Birch PR (2013) An RxLR effector from Phytophthora infestans prevents re-localisation of two plant NAC transcription factors from the endoplasmic reticulum to the nucleus. PLOS Pathog 9: e1003670.

Mhamdi A, Queval G, Chaouch S, Vanderauwera S, Van Breusegem F, Noctor G (2010) Catalase function in plants: a focus on Arabidopsis mutants as stress-mimic models. J Exp Bot 61: 4197-4220.

Michielse CB, Rep M (2009) Pathogen profile update: Fusarium oxysporum. Mol Plant Pathol 10: 311-324.

Misas-Villamil JC, Hoorn RA, Doehlemann G (2016) Papain-like cysteine proteases as hubs in plant immunity. New Phytol 212: 902-907.

Murphy E, De Smet I (2014) Understanding the RALF family: a tale of many species. Trends Plant Sci 19: 664-671. 


\section{Chapter 2}

Notz R, Maurhofer M, Dubach H, Haas D, Défago G (2002) Fusaric acid-producing strains of Fusarium oxysporum alter 2, 4-diacetylphloroglucinol biosynthetic gene expression in Pseudomonas fluorescens CHA0 in vitro and in the rhizosphere of wheat. Appl Environ Microbiol 68: 2229-2235.

Novo M, Pomar F, Gayoso C, Merino F (2006) Cellulase activity in isolates of Verticillium dahliae differing in aggressiveness. Plant Dis 90: 155-160.

Oome S, Raaymakers TM, Cabral A, Samwel S, Böhm H, Albert I, Nürnberger T, Van den Ackerveken G (2014) Nep1-like proteins from three kingdoms of life act as a microbe-associated molecular pattern in Arabidopsis. Proc Natl Acad Sci USA 111: 16955-16960.

Oome S, Van den Ackerveken G (2014) Comparative and functional analysis of the widely occurring family of nep1-like proteins. Mol Plant-Microbe Interact 27: 1081-1094.

Ospina-Giraldo MD, Mullins E, Kang S (2003) Loss of function of the Fusarium oxysporum SNF1 gene reduces virulence on cabbage and Arabidopsis. Curr Genet 44: 49-57.

Otten L, Burr T, Szegedi E (2008) Agrobacterium: a disease-causing bacterium. In: Tzfira T, Citovsky V (eds) Agrobacterium: from biology to biotechnology. Springer, New York, NY.

Ottmann C, Luberacki B, Küfner I, Koch W, Brunner F, Weyand M, Mattinen L, Pirhonen M, Anderluh G, Seitz HU (2009) A common toxin fold mediates microbial attack and plant defense. Proc Natl Acad Sci USA 106: 10359-10364.

Raaijmakers JM, Paulitz TC, Steinberg C, Alabouvette C, Moënne-Loccoz Y (2009) The rhizosphere: a playground and battlefield for soilborne pathogens and beneficial microorganisms. Plant Soil 321: 341-361.

Peeters N, Guidot A, Vailleau F, Valls M (2013) Ralstonia solanacearum, a widespread bacterial plant pathogen in the post-genomic era. Mol Plant Pathol 14: 651-662.

Pieterse CM, Van der Does D, Zamioudis C, Leon-Reyes A, Van Wees SC (2012) Hormonal modulation of plant immunity. Annu Rev Cell Dev Biol 28: 489-521.

Poueymiro M, Genin S (2009) Secreted proteins from Ralstonia solanacearum: a hundred tricks to kill a plant. Curr Opin Microbiol 12: 44-52.

Prusky D, Yakoby N (2003) Pathogenic fungi: leading or led by ambient pH? Mol Plant Pathol 4: 509-516.

Qiao Y, Liu L, Xiong Q, Flores C, Wong J, Shi J, Wang X, Liu X, Xiang Q, Jiang S, Zhang F, Wang Y, Judelson H, Chen X, Ma W (2013) Oomycete pathogens encode RNA silencing suppressors. Nat Genet 45: 330-333.

Qiao Y, Shi J, Zhai Y, Hou Y, Ma W (2015) Phytophthora effector targets a novel component of small RNA pathway in plants to promote infection. Proc Natl Acad Sci USA 112: 5850-5855.

Qutob D, Kemmerling B, Brunner F, Küfner I, Engelhardt S, Gust AA, Luberacki B, Seitz HU, Stahl D, Rauhut T (2006) Phytotoxicity and innate immune responses induced by Nep1-like proteins. Plant Cell 18: 3721-3744.

Raffaele S, Kamoun S (2012) Genome evolution in filamentous plant pathogens: why bigger can be better. Nat Rev Microbiol 10: 417-430.

Raffaele S, Win J, Cano LM, Kamoun S (2010) Analyses of genome architecture and gene expression reveal novel candidate virulence factors in the secretome of Phytophthora infestans. BMC Genomics 11: 637 .

Rajput NA, Zhang M, Ru Y, Liu T, Xu J, Liu L, Mafurah JJ, Dou D (2014) Phytophthora sojae effector PsCRN70 suppresses plant defenses in Nicotiana benthamiana. PLOS ONE 9: e98114.

Reddy PP (2013) Recent advances in crop protection. Springer, Heidelberg.

Remigi P, Anisimova M, Guidot A, Genin S, Peeters N (2011) Functional diversification of the GALA type III effector family contributes to Ralstonia solanacearum adaptation on different plant hosts. New Phytol 192: 976-987. 
Rooney HC, van't Klooster JW, van der Hoorn RA, Joosten MH, Jones JD, de Wit PJM (2005) Cladosporium Avr2 inhibits tomato Rcr3 protease required for Cf-2-dependent disease resistance. Science 308: 1783-1786.

Rose JK, Ham K-S, Darvill AG, Albersheim P (2002) Molecular cloning and characterization of glucanase inhibitor proteins coevolution of a counterdefense mechanism by plant pathogens. Plant Cell 14: 1329-1345.

Rovenich H, Boshoven JC, Thomma BPHJ (2014) Filamentous pathogen effector functions: of pathogens, hosts and microbiomes. Curr Opin Plant Biol 20: 96-103.

Rovenich H, Zuccaro A, Thomma BPHJ (2016) Convergent evolution of filamentous microbes towards evasion of glycan-triggered immunity. New Phytol 212: 896-901.

Rushton PJ, Somssich IE, Ringler P, Shen QJ (2010) WRKY transcription factors. Trends Plant Sci 15: 247-258.

Sánchez-Rangel D, Sánchez-Nieto S, Plasencia J (2012) Fumonisin B1, a toxin produced by Fusarium verticillioides, modulates maize $\beta$-1,3-glucanase activities involved in defense response. Planta 235 : 965-978.

Sánchez-Vallet A, Mesters JR, Thomma BPHJ (2015) The battle for chitin recognition in plant-microbe interactions. FEMS Microbiol Rev 39: 171-183.

Sánchez-Vallet A, Saleem-Batcha R, Kombrink A, Hansen G, Valkenburg D-J, Thomma BPHJ, Mesters JR (2013) Fungal effector Ecp6 outcompetes host immune receptor for chitin binding through intrachain LysM dimerization. eLife 2: e00790.

Santhanam P, van Esse HP, Albert I, Faino L, Nürnberger T, Thomma BPHJ (2013) Evidence for functional diversification within a fungal NEP1-like protein family. Mol Plant-Microbe Interact 26: 278-286.

Sarris PF, Duxbury Z, Huh SU, Ma Y, Segonzac C, Sklenar J, Derbyshire P, Cevik V, Rallapalli G, Saucet SB, Wirthmueller L, Menke FL, Sohn KH, Jones JD (2015) A plant immune receptor detects pathogen effectors that target WRKY transcription factors. Cell 161: 1089-1100.

Schnider-Keel U, Seematter A, Maurhofer M, Blumer C, Duffy B, Gigot-Bonnefoy C, Reimmann C, Notz R, Défago G, Haas D (2000) Autoinduction of 2, 4-diacetylphloroglucinol biosynthesis in the biocontrol agent Pseudomonas fluorescens CHA0 and repression by the bacterial metabolites salicylate and pyoluteorin. J Bacteriol 182: 1215-1225.

Schornack S, van Damme M, Bozkurt T0, Cano LM, Smoker M, Thines M, Gaulin E, Kamoun S, Huitema E (2010) Ancient class of translocated oomycete effectors targets the host nucleus. Proc Natl Acad Sci USA 107: 17421-17426.

Schouten A, van den Berg G, Edel-Hermann V, Steinberg C, Gautheron N, Alabouvette C, De Vos C, Lemanceau P, Raaijmakers JM (2004) Defense responses of Fusarium oxysporum to 2, 4diacetylphloroglucinol, a broad-spectrum antibiotic produced by Pseudomonas fluorescens. Mol Plant-Microbe Interact 17: 1201-1211.

Schrammeijer B, Risseeuw E, Pansegrau W, Regensburg-Tuïnk TJ, Crosby WL, Hooykaas PJ (2001) Interaction of the virulence protein VirF of Agrobacterium tumefaciens with plant homologs of the yeast Skp1 protein. Curr Biol 11: 258-262.

Schwelm A, Fogelqvist J, Knaust A, Jülke S, Lilja T, Bonilla-Rosso G, Karlsson M, Shevchenko A, Dhandapani V, Choi SR (2015) The Plasmodiophora brassicae genome reveals insights in its life cycle and ancestry of chitin synthases. Sci Rep 5: 11153.

Seidl MF, Thomma BPHJ (2014) Sex or no sex: evolutionary adaptation occurs regardless. BioEssays 36: 335-345. 
Song J, Win J, Tian M, Schornack S, Kaschani F, Ilyas M, van der Hoorn RA, Kamoun S (2009) Apoplastic effectors secreted by two unrelated eukaryotic plant pathogens target the tomato defense protease Rcr3. Proc Natl Acad Sci 106: 1654-1659.

Sperschneider J, Dodds PN, Gardiner DM, Manners JM, Singh KB, Taylor JM (2015) Advances and challenges in computational prediction of effectors from plant pathogenic fungi. PLOS Pathog 11: e1004806.

Sperschneider J, Gardiner DM, Dodds PN, Tini F, Covarelli L, Singh KB, Manners JM, Taylor JM (2016) EffectorP: predicting fungal effector proteins from secretomes using machine learning. New Phytol 210: 743-761.

Stam R, Motion G, Boevink PC, Huitema E (2013) A conserved oomycete CRN effector targets and modulates tomato TCP14-2 to enhance virulence. bioRxiv doi: https://doi.org/10.1101/001248.

Stergiopoulos I, de Wit PJM (2009) Fungal effector proteins. Annu Rev Phytopathol 47: 233-263

Stirnberg A, Djamei A (2016) Characterization of ApB73, a virulence factor important for colonization of Zea mays by the smut Ustilago maydis. Mol Plant Pathol 17: 1467-1479.

Sun Y, Li P, Deng M, Shen D, Dai G, Yao N, Lu Y (2017) The Ralstonia solanacearum effector RipAK suppresses plant hypersensitive response by inhibiting the activity of host catalases. Cell Microbiol doi: $10.1111 / \mathrm{cmi} .12736$.

Thomma BPHJ, Nürnberger T, Joosten MH (2011) Of PAMPs and effectors: the blurred PTI-ETI dichotomy. Plant Cell 23: 4-15.

Thynne E, Saur IM, Simbaqueba J, Ogilvie HA, Gonzalez-Cendales Y, Mead O, Taranto A, Catanzariti AM, McDonald MC, Schwessinger B (2016) Fungal phytopathogens encode functional homologues of plant rapid alkalinization factor (RALF) peptides. Mol Plant Pathol 18: 811-824.

Tian M, Benedetti B, Kamoun S (2005) A second Kazal-like protease inhibitor from Phytophthora infestans inhibits and interacts with the apoplastic pathogenesis-related protease P69B of tomato. Plant Physiol 138: 1785-1793.

Tian M, Huitema E, Da Cunha L, Torto-Alalibo T, Kamoun S (2004) A Kazal-like extracellular serine protease inhibitor from Phytophthora infestans targets the tomato pathogenesis-related protease P69B. J Bio Chem 279: 26370-26377.

Tian M, Win J, Song J, van der Hoorn R, van der Knaap E, Kamoun S (2007) A Phytophthora infestans cystatin-like protein targets a novel tomato papain-like apoplastic protease. Plant Physiol 143: 364377.

Toruño TY, Stergiopoulos I, Coaker G (2016) Plant-pathogen effectors: cellular probes interfering with plant defenses in spatial and temporal manners. Annu Rev Phytopathol 54: 419-441.

Tyler BM, Tripathy S, Zhang X, Dehal P, Jiang RH, Aerts A, Arredondo FD, Baxter L, Bensasson D, Beynon JL (2006) Phytophthora genome sequences uncover evolutionary origins and mechanisms of pathogenesis. Science 313: 1261-1266.

Tzfira T, Vaidya M, Citovsky V (2004) Involvement of targeted proteolysis in plant genetic transformation by Agrobacterium. Nature 431: 87-92.

Tzima AK, Paplomatas EJ, Rauyaree P, Ospina-Giraldo MD, Kang S (2011) VdSNF1, the sucrose nonfermenting protein kinase gene of Verticillium dahliae, is required for virulence and expression of genes involved in cell-wall degradation. Mol Plant-Microbe Interact 24: 129-142.

van den Burg HA, Harrison SJ, Joosten MH, Vervoort J, de Wit PJM (2006) Cladosporium fulvum Avr4 protects fungal cell walls against hydrolysis by plant chitinases accumulating during infection. Mol Plant-Microbe Interact 19: 1420-1430. 
van Esse HP, van't Klooster JW, Bolton MD, Yadeta KA, van Baarlen P, Boeren S, Vervoort J, de Wit PJM, Thomma BPHJ (2008) The Cladosporium fulvum virulence protein Avr2 inhibits host proteases required for basal defense. Plant Cell 20: 1948-1963.

van West P, Appiah AA, Gow NA (2003) Advances in research on oomycete root pathogens. Physiol Mol Plant Pathol 62: 99-113.

Vance V, Vaucheret H (2001) RNA silencing in plants--defense and counterdefense. Science 292: 22772280.

Vleeshouwers VG, Oliver RP (2014) Effectors as tools in disease resistance breeding against biotrophic, hemibiotrophic, and necrotrophic plant pathogens. Mol Plant-Microbe Interact 27: 196-206.

Wang M, Weiberg A, Lin F-M, Thomma BPHJ, Huang H-D, Jin H (2016) Bidirectional cross-kingdom RNAi and fungal uptake of external RNAs confer plant protection. Nat Plants 2: 16151.

Weiberg A, Wang M, Lin F-M, Zhao H, Zhang Z, Kaloshian I, Huang H-D, Jin H (2013) Fungal small RNAs suppress plant immunity by hijacking host RNA interference pathways. Science 342: 118-123

Whisson SC, Boevink PC, Moleleki L, Avrova AO, Morales JG, Gilroy EM, Armstrong MR, Grouffaud S, Van West P, Chapman S (2007) A translocation signal for delivery of oomycete effector proteins into host plant cells. Nature 450: 115-118.

Williams AH, Sharma M, Thatcher LF, Azam S, Hane JK, Sperschneider J, Kidd BN, Anderson JP, Ghosh R, Garg G (2016) Comparative genomics and prediction of conditionally dispensable sequences in legume-infecting Fusarium oxysporum formae speciales facilitates identification of candidate effectors. BMC Genomics 17: 191.

Wu C-H, Yan H-Z, Liu L-F, Liou R-F (2008) Functional characterization of a gene family encoding polygalacturonases in Phytophthora parasitica. Mol Plant-Microbe Interact 21: 480-489.

Yadeta KA, Thomma BPHJ (2013) The xylem as battleground for plant hosts and vascular wilt pathogens. Front Plant Sci 4: 97.

Zhang M, Li Q, Liu T, Liu L, Shen D, Zhu Y, Liu P, Zhou JM, Dou D (2015) Two cytoplasmic effectors of Phytophthora sojae regulate plant cell death via interactions with plant catalases. Plant Physiol 167: 164-175.

Zhang Z, Fradin E, de Jonge R, van Esse HP, Smit P, Liu C-M, Thomma BPHJ (2013) Optimized agroinfiltration and virus-induced gene silencing to study Ve1-mediated Verticillium resistance in tobacco. Mol Plant-Microbe Interact 26: 182-190.

Zhao Z, Liu H, Wang C, Xu J-R (2013) Comparative analysis of fungal genomes reveals different plant cell wall degrading capacity in fungi. BMC Genomics 14: 274.

Zhou B-J, Jia P-S, Gao F, Guo H-S (2012) Molecular characterization and functional analysis of a necrosisand ethylene-inducing, protein-encoding gene family from Verticillium dahliae. Mol Plant-Microbe Interact 25: 964-975. 



\section{Chapter 3}

\section{Tomato immune receptor Ve1 recognizes co-localized $\mathrm{N}$ - and C-termini of Verticillium dahliae effector Ave1}

Yin Song1\#, Zhao Zhang1,2,5\#, Jordi C. Boshoven¹, Hanna Rovenich¹, Michael F. Seidl ${ }^{1}$, Jernej Jakše³, Karunakaran Maruthachalam4, Chun-Ming Liu², Krishna V. Subbarao4, Branka Javornik ${ }^{3}$, and Bart P.H.J. Thomma ${ }^{1}$

${ }^{1}$ Laboratory of Phytopathology, Wageningen University, Droevendaalsesteeg 1, 6708 PB Wageningen, the Netherlands

${ }^{2}$ Key Laboratory of Plant Molecular Physiology, Institute of Botany, the Chinese Academy of Sciences, Beijing 100093, China

${ }^{3}$ Department of Agronomy, Biotechnical Faculty, University of Ljubljana, Jamnikarjeva 101, SI-1000 Ljubljana, Slovenia ${ }^{4}$ Department of Plant Pathology, University of California, Davis, CA 95616, USA 5Present address: Department of Ornamental Horticulture and Landscape Architecture, China Agricultural University, Beijing 100193, China

A preprint of this chapter has been deposited as:

Song Y\#, Zhang Z\#, Boshoven J, Rovenich H, Seidl M, Jakse J, Maruthachalam K, Liu C-M, Subbarao K, Javornik B, Thomma BPHJ (2017) Tomato immune receptor Ve1 recognizes surface-exposed colocalized $\mathrm{N}$ - and C-termini of Verticillium dahliae effector Ave1. bioRxiv doi: https://doi.org/10.1101/103473. ("equal contribution) 


\section{Chapter 3}

\section{ABSTRACT}

Effectors are secreted by plant pathogens to facilitate infection, often through deregulation of plant immune responses. During host colonization, race 1 strains of the soil-borne vascular wilt fungus Verticillium dahliae secrete the effector protein Ave1 that triggers immunity in tomato genotypes that encode the cell surfacelocalized immune receptor Ve1. Homologs of $V$. dahliae Ave1 (VdAve1) are found in plants and in few plant pathogenic microbes, and are differentially recognized by Ve1. However, how VdAve1 is recognized by Ve1 remained unknown. Interestingly, C-terminally affinity-tagged versions of VdAve1 failed to activate Ve1-mediated immunity, suggesting that exposure of the C-terminus of VdAve1 is required for Ve1-mediated recognition. This was confirmed by subsequent analyses of Cterminal deletion mutants, and by amino acids swap experiments. Although required, only the $\mathrm{C}$-terminus of VdAve1 is not sufficient to activate Ve1-mediated immunity. Further analyses of N-terminal deletion mutants revealed that also the $\mathrm{N}$-terminus of VdAve1 is required to activate Ve1-mediated immunity. Intriguingly, a three-dimensional structural model of VdAve1 revealed that the $\mathrm{N}$ - and C-termini co-localize on a surface-exposed patch of the VdAve1 protein. Thus, we propose that a surface-exposed patch of the VdAve1 protein that is composed of co-localized $\mathrm{N}$ - and C-termini is recognized by the tomato cell-surface immune receptor Ve1. 


\section{INTRODUCTION}

Plants are constantly engaged in battles against diverse groups of microbes within their environment. However, only few of these actually become pathogens to cause disease, as plants have developed innate immunity to protect themselves against microbial attack (Dodds and Rathjen, 2010; Thomma et al., 2011; Cook et al., 2015). In its simplest form, plant immunity against pathogen attack is governed by immune receptors that sense pathogen-derived(induced) ligands to activate defense. Originally, the interaction between plant immune receptors and pathogen ligands was described in the "gene-for-gene" model, stating that the products of plant resistance $(R)$ genes induce race-specific resistance upon recognition of the products of corresponding pathogen avirulence (Avr) genes (Flor, 1971). Decades later, an updated view of plant innate immunity has been introduced as the "zigzag" model, by incorporating pathogen-secreted effector molecules that suppress host immune responses, but that may subsequently be recognized by newly evolved immune receptors, in turn (Jones and Dangl, 2006). In this model, the first line of defense is governed by plasma membrane-localized pattern recognition receptors (PRRs) that detect conserved microbe-associated molecular patterns (MAMPs) to activate MAMP-triggered immunity (MTI). In subsequent layers of defense, effectors are recognized by the corresponding resistance proteins (R proteins), resulting in effector-triggered immunity (ETI). Although initially portrayed as separate layers, numerous studies on various plant-microbe interactions have revealed that the delineation between MTI and ETI is not strict, but rather a continuum (Thomma et al., 2011; Cook et al., 2015). Moreover, the fact that MAMPs are defined from the perspective of the host, whereas effectors are defined from the perspective of the invader, creates a conceptual conflict and has recently inspired the formulation of the Invasion Model, in which host receptors (termed invasion pattern receptors; IPRs) detect either externally encoded or modified-self ligands (termed invasion patterns; IPs) that betray invasion (Cook et al., 2015). In this model, any molecule can serve as an IP that is detected by an IPR, but the probability of a particular ligand-receptor complex to evolve within the framework of host immunity increases with increasing ligand probability to retain function, conservation across organisms, importance in establishment of symbiosis, and accessibility (Cook et al., 2015). 


\section{Chapter 3}

Verticillium dahliae is a xylem invading fungal pathogen that causes Verticillium wilt diseases in a wide range of plant species worldwide (Fradin and Thomma, 2006). V. dahliae persists in the soil and enters plants through their roots. Once inside the root, the fungus grows intercellularly and invades the xylem vessels, where it sporulates to spread through the vascular system. Typical symptoms of $V$. dahliae infection include stunting, wilting, chlorosis, and necrosis (Fradin and Thomma, 2006). In tomato (Solanum lycopersicum), a single dominant locus that confers Verticillium resistance has been identified as the Ve locus, which controls Verticillium isolates that are assigned to race 1 (Schaible et al., 1951). The Ve locus comprises two closely linked and inversely oriented genes, $V e 1$ and $V e 2$, that both encode extracellular leucine-rich repeat (eLRR) receptor-like proteins (RLPs) (Kawchuk et al., 2001; Wang et al., 2010). Of these, only Ve1 was found to confer resistance against race 1 isolates of Verticillium in tomato (Fradin et al., 2009). Intriguingly, interfamily transfer of Ve1 from tomato to Arabidopsis thaliana has resulted in race-specific Verticillium resistance in the latter species (Fradin et al., 2011, 2014; Zhang et al., 2014), implying that the underlying immune signalling cascade across plant taxonomy is evolutionarily conserved (Fradin et al., 2011; Thomma et al., 2011). Moreover, homologs of tomato Ve1 that have the potential to recognize race 1 strains of $V$. dahliae have been characterized in other plant species, including tobacco, potato, wild eggplant and hop, suggesting an ancient origin of the tomato immune receptor Ve1 (Song et al., 2017).

Through comparative population genomics, the $V$. dahliae effector protein that is recognized by the tomato cell surface-localized immune receptor Ve1 was

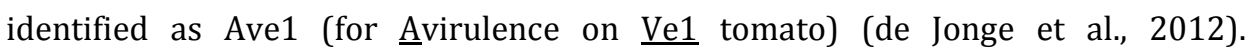
Interestingly, homologs of $V$. dahliae Ave1 (VdAve1) were identified from a number of fungal pathogens, including the tomato pathogen Fusarium oxysporum f. sp. lycopersici (FoAve1), the sugar beet pathogen Cercospora beticola (CbAve1) and the crucifer pathogen Colletotrichum higginsianum (ChAve1) (de Jonge et al., 2012). Strikingly, however, most VdAve1 homologs were found in plants, with the most closely related homologs derived from tomato (S. lycopersicum; SIPNP) and grape (Vitis vinifera; VvPNP) (de Jonge et al., 2012). Finally, a more distantly related homolog was identified in the plant pathogenic bacterium Xanthomonas axonopodis pv. citri (XacPNP) (de Jonge et al., 2012). Co-expression of VdAve1, SIPNP, FoAve1, 
and CbAve1 with tomato Ve1 in tobacco triggers a hypersensitive response (HR), whereas co-expression of ChAve1 with tomato Ve1 does not lead to an HR (de Jonge et al., 2012). Moreover, Ve1 was found to mediate resistance towards F. oxysporum in tomato, demonstrating involvement of the tomato immune receptor Ve1 in resistance against multiple fungal pathogens (de Jonge et al., 2012).

Many eLRR-containing cell-surface immune receptors recognize peptide sequences as epitopes of their pathogen ligands. For example, flg22 is the 22amino-acid peptide derived from bacterial flagellin that is perceived by the receptor-like kinase (RLK)-type immune receptor FLS2 (Zipfel et al., 2004), while the Arabidopsis RLK-type EFR immune receptor was shown to recognize elf18, an 18-amino-acid peptide derived from bacterial EF-Tu (Zipfel et al., 2006). Similarly, a highly conserved 22-amino-acid sequence derived from the bacterial cold shock protein, named csp22, is perceived by the tomato RLK-type immune receptor CORE (Felix and Boller, 2003; Wang et al., 2016), while a surface-exposed pentapeptide TKLGE of the $22 \mathrm{kDa}$ ethylene-inducing xylanase (EIX) from the biocontrol fungus Trichoderma viride determines recognition by the RLP-type receptor LeEIX2 in tomato (Rotblat et al., 2002; Ron and Avni, 2004). Furthermore, the tyrosinesulfated 21-amino-acid peptide RaxX21-sY of Xanthomonas oryzae pv. oryzae RaxX is sufficient to activate XA21-mediated immunity in rice (Pruitt et al., 2015). Finally, a conserved 20-amino-acid fragment present in most Nep1-like proteins (NLPs) (nlp20) is sufficient to activate RLP23-mediated immunity in Arabidopsis (Böhm et al., 2014; Oome et al., 2014; Albert et al., 2015). Here, we aimed to identify a minimal motif in the $V$. dahliae effector protein VdAve1 that is necessary and sufficient for recognition by the tomato cell-surface immune receptor Ve1. Our approach was based on epitope prediction, guided by the alignment of differentially recognized VdAve1 homologs, followed by functional analyses of a combination of serial deletion assays, amino acids swaps, synthetic peptides, chimeric proteins and three-dimensional modelling.

\section{RESULTS}

\section{Sequence conservation among Ave1 homologs}

Previously, we reported the cloning of Ave1 from $V$. dahliae and described the absence of allelic variation among 85 Ave1 alleles derived from $V$. dahliae and $V$. 


\section{Chapter 3}

albo-atrum (de Jonge et al., 2012). Ave1 alleles were not identified in any of the $V$. dahliae and V. albo-atrum race 2 strains analysed, nor in strains that are not pathogenic on tomato, nor in V. longisporum or V. tricorpus (de Jonge et al., 2012). To further explore Ave1 diversity, we assessed presence in a collection of 129 Verticillium strains isolated from various host plants and different geographical locations, resulting in the identification of 22 novel Ave1 alleles (Table S1). No allelic variation was found among the newly identified Ave1 alleles amplified from $V$. dahliae as well as from V. alfalfae and V. nonalfalfae, two species that have recently been recognized as novel species distinct from $V$. albo-atrum (Inderbitzin et al., 2011). However, a VdAve1 homolog was identified in four isolates of $V$. nubilum (VnAve1), a species that is known as a saprophyte and opportunistic pathogen (Isaac, 1953). While the predicted VnAve1 protein sequence displays 13 amino acid polymorphisms when compared with VdAve1 (Figure 1A), the four VnAve1 alleles were found to be identical to each other.

Alignment of the amino acid sequences of VdAve1 and its homologs from plants (SIPNP and VvPNP) and plant pathogens (VnAve1, FoAve1, CbAve1, ChAve1 and XacPNP) revealed blocks of highly conserved amino acids that are alternated with more variable regions (Figure 1A). Based on prediction by SignalP 4.1 (Petersen et al., 2011), all VdAve1 homologs contain a predicted N-terminal signal peptide that directs secretion into the extracellular space (Figure 1A; D-cutoff score $>0.6$ ). Moreover, the four cysteine residues that are present in VdAve1 are conserved among all homologs (Figure 1A), and in silico analysis using DISULFIND (Ceroni et al., 2006) suggests the formation of disulphide bridges between Cys ${ }^{35}$ and $\mathrm{Cys}^{63}$, as well as between $\mathrm{Cys}^{71}$ and $\mathrm{Cys}^{79}$. From the alignment it is apparent that XacPNP is the most divergent, while all other VdAve1 homologs are relatively similar (Figure 1A).

\section{Comparison of the necrosis-inducing activity of VdAve1 homologs}

It was previously demonstrated that tomato Ve1 recognizes not only VdAve1, but also SIPNP, FoAve1 and CbAve1 (de Jonge et al., 2012). We now also tested the necrosis-inducing capacity of VnAve1, VvPNP and XacPNP that were isolated from $V$. nubilum, V. vinifera and $X$. axonopodis, respectively. Co-expression of the sequenceunrelated effector Avr9 from the tomato leaf mould fungus Cladosporium fulvum 


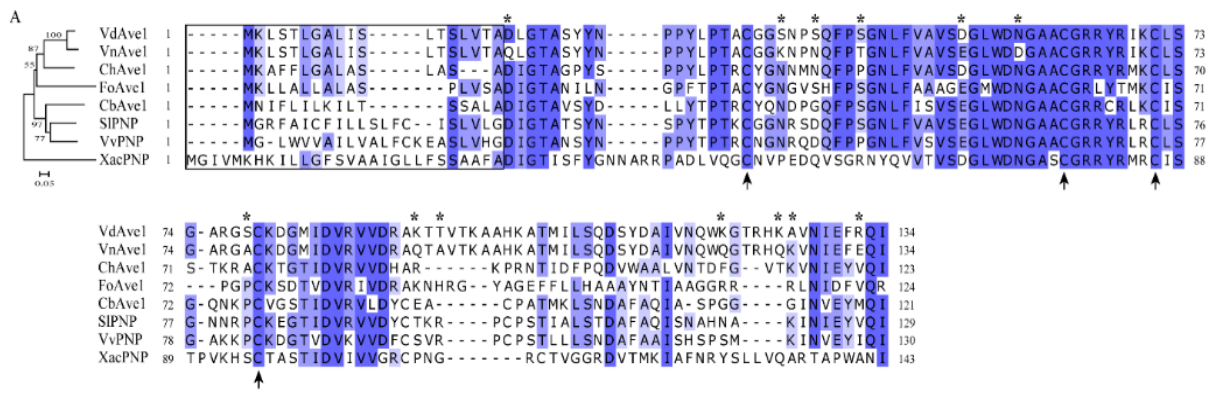

B
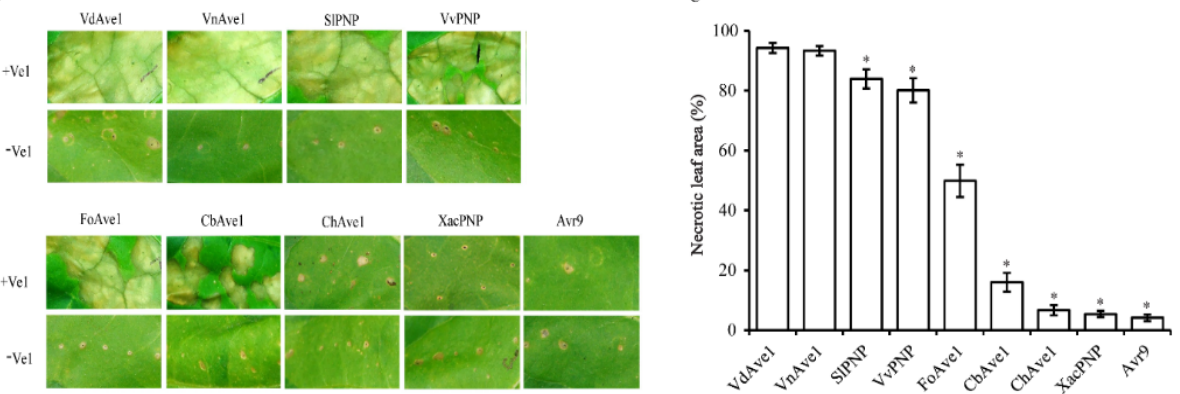

Figure 1. Distinct necrosis induced by VdAve1 homologs through co-expression with tomato Ve1 in Nicotiana tabacum. (A) Amino acid sequence alignment of VdAve1 homologs from Verticillium dahliae (VdAve1), V. nubilum (VnAve1), Solanum lycopersicum (SIPNP), Vitis vinifera (VvPNP), Fusarium oxysporum f. sp. lycopersici (FoAve1), Cercospora beticola (CbAve1), Colletotrichum higginsianum (ChAve1), and Xanthomonas axonopodis pv. citri (XacPNP). Phylogeny of VdAve1 and its homologs described above is shown. The bootstrap percentage support for each branch is indicated. The scale bar represents $5 \%$ weighted amino acid sequence divergence. Blue shade background indicates identical amino acids while the color intensity represents the frequency. Asterisks indicate the 13 amino acid polymophisms between VdAve1 and VnAve1. The positions of four conserved cysteine residues are indicated with arrows in the bottom. The N-terminal amino acids in the frame denote the predicted signal peptides (SP) of the VdAve1 homologs. (B) Coexpression of Ve1 and Ave1 homologs VdAve1, VnAve1, SIPNP, VvPNP, FoAve1, CbAve1, ChAve1, and XacPNP in N. tabacum. Expression of the sequence-unrelated effector Avr 9 from the tomato leaf mold fungus Cladosporium fulvum in combination with $V e 1$ or absence of $V e 1$ are shown as negative controls. Pictures were taken at five days post infiltration (dpi). (C) Quantification of necrosis resulting from recognition of VdAve1 homologs by Ve1 at 5 dpi ( $\mathrm{n}>20$ ). Bars represent the average percentage of necrotic leaf area of infiltration zones with standard deviations. Asterisks above the top of the bars represent statistically significant differences when compared with VdAve1-indcued necrosis $(P<0.05)$.

(van Kan et al., 1991) with Ve1 served as a negative control. Whereas expression of VnAve1 or VvPNP together with Ve1 in Nicotiana tabacum resulted in strong HR, coexpression of XacPNP or Avr9 with Ve1 triggered little to no necrosis in addition to the small wounds that were caused by the infiltration procedure (Figure 1B). To 


\section{Chapter 3}

compare the necrosis induced by the various VdAve1 homologs, they were coexpressed with Ve1 in N. tabacum and HR development was measured by quantification of the leaf area that developed necrosis at five days post infiltration (dpi). Importantly, none of the VdAve1 homologs induced necrosis in the absence of Ve1 (Figure 1B). Whereas agroinfiltration of either VdAve1 or VnAve1 with Ve1 resulted in complete necrosis of the infiltrated leaf area, agroinfiltration of FoAve1 with $V e 1$ resulted in large necrotic spots in the infiltrated leaf area, although no complete collapse of the infiltrated area was observed (Figure 1B, C). Upon agroinfiltration of CbAve1 with Ve1, spreading of smaller and larger necrotic spots was observed in all infiltrated areas, but the infiltrated leaf area did not turn completely necrotic. For ChAve1, XacPNP and Avr9, necrosis did not extend beyond the wounded infiltration sites (Figure 1B, C). Upon agroinfiltration of the tomato and grape VdAve1 homologs, SIPNP and $V v P N P$, most of the infiltrated leaf area developed necrosis (Figure 1B, C), occasionally affecting the complete infiltrated leaf sector. To confirm that variable levels of HR induced by VdAve1 homologs are not due to instability of the VdAve1 homologs in planta, GFP-tagged VdAve1 homologs were detected by immunoblotting. Similar to GFP-tagged VdAve1 protein or GFP-tagged VnAve1, all other GFP-tagged VdAve1 homologs accumulated to clearly detectable protein levels in planta (Figure S1).

\section{The C-terminus of VdAve1 is required, but not sufficient, for recognition by tomato Ve1}

In order to perform further functional analyses, a construct encoding C-terminally GFP-tagged VdAve1 (VdAve1_c GFP) was generated. However, C-terminal fusion of a GFP tag to VdAve1 resulted in loss of recognition by Ve1 (Figure 2A, C). Considering that the GFP tag $(\sim 27 \mathrm{kDa})$ is relatively large, we engineered $\mathrm{C}$ terminally tagged VdAve1 fusions with smaller protein tags $(<12 \mathrm{kDa}$; VdAve1_c 3xHA, VdAve1_c 6xHis, VdAve1_c 4xMyc, VdAve1_c 10xMyc and VdAve1_c FLAG; Figure 2A). Despite their smaller size, all additionally tested C-terminal tags abolished, or significantly reduced, HR development on Ve1-expressing tobacco leaves (Figure 2A, C). Importantly, the C-terminally GFP-tagged VdAve1 fusion was clearly detected by immunoblotting (Figure S2A), suggesting that accessibility of the VdAve1 C-terminus is important in VdAve1 recognition by Ve1. 

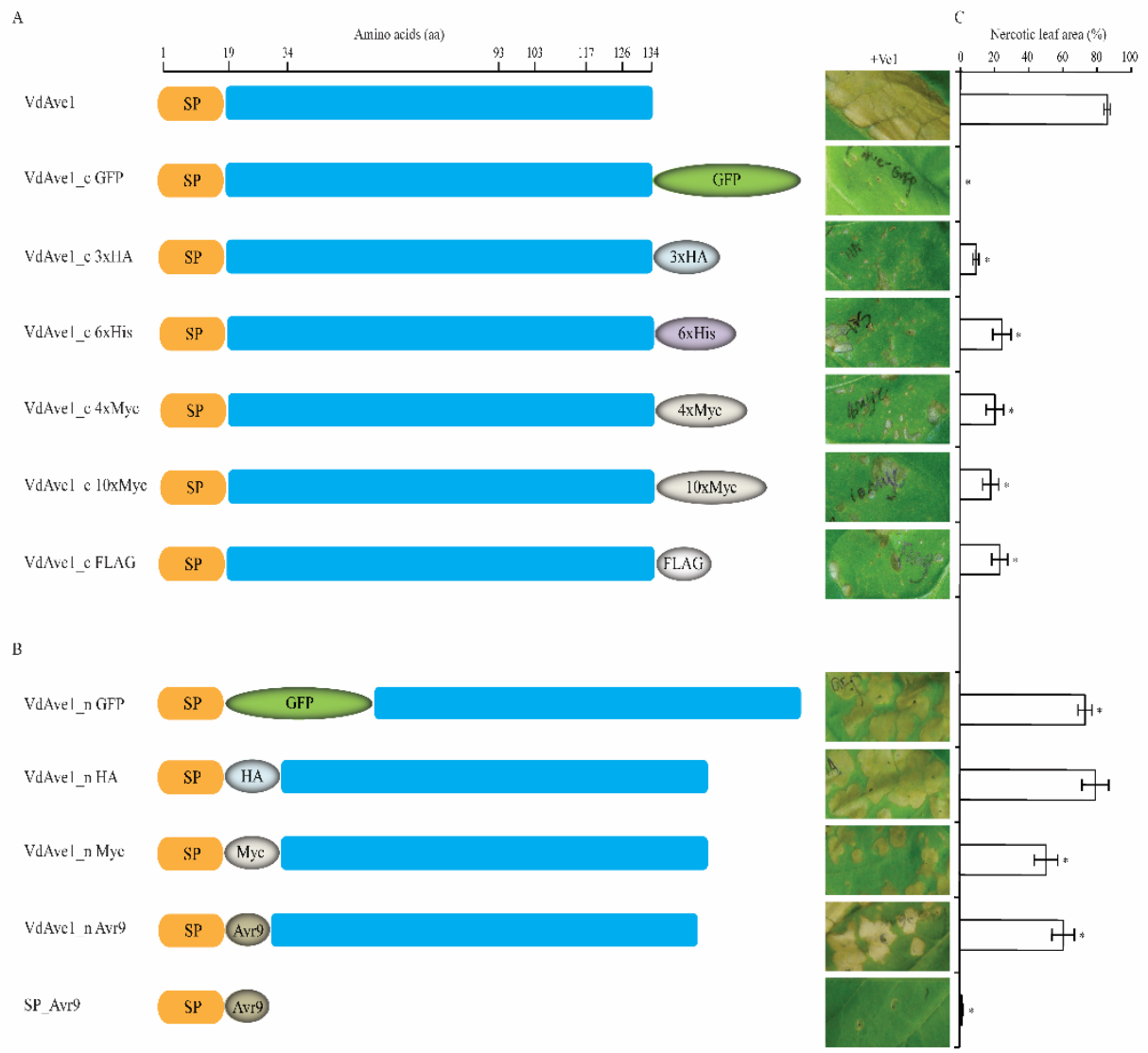

Figure 2. Effect of C- or $\mathrm{N}$ - terminally tagged VdAve1 versions on recognition of VdAve1 by Ve1 in tobacco. (A) Constructs encoding full-length VdAve1 (VdAve1) and C-terminally tagged VdAve1 versions (VdAve1_c GFP, VdAve1_c 3xHA, VdAve1_c 6xHis, VdAve1_c 4xMyc, VdAve1_c 10xMyc, VdAve1_c FLAG) were assayed for necrosis-inducing capability. The constructs were co-expressed with $V e 1$ in tobacco respectively, and the occurrence of necrosis was monitored at $5 \mathrm{dpi}$. The feature includes a predicated signal peptide (SP) involved in effector secretion. (B) Constructs encoding $\mathrm{N}$-terminally fused VdAve1 versions (VdAve1_n GFP, VdAve1_n HA, VdAve1_n Myc and VdAve1_n Avr9) were assayed for necrosis-inducing capability. The constructs were co-expressed with $V e 1$ in tobacco respectively, and the necrosis occurrence was monitored at $5 \mathrm{dpi}$. A construct encoding mature Avr9 fused with VdAve1 signal peptide (SP_Avr9) co-expressed with Ve1 is used as a negative control. (C) Quantification of necrosis resulting from recognition of tagged VdAve1 proteins by Ve1 at 5 dpi. The graph shows the average percentage of necrotic leaf area of infiltration zones at $5 \mathrm{dpi}(\mathrm{n}>5)$. Data are presented as mean with standard deviations. Asterisks indicate statistically significant differences when compared with wild-type VdAve1-indcued necrosis $(P<0.05)$.

To further investigate the role of the C-terminus in recognition of VdAve1 by Ve1, a number of C-terminal truncation mutants were generated. Deletion of 42 


\section{Chapter 3}

amino acids from the C-terminus (Lys ${ }^{93}$ to Ile ${ }^{134}$; VdAve1 ${ }^{493-134}$ ) resulted in loss of VdAve1 recognition by Ve1 (Figure 3A, B). Subsequent analysis of step-wise smaller truncations revealed that a C-terminal deletion of nine amino acids (VdAve1 ${ }^{\Delta 126-134}$ ) resulted in loss of Ve1-mediated recognition (Figure 3A, B), even though the presence of GFP-tagged VdAve1 $1^{\Delta 126-134}$ protein in planta was verified by immunoblotting (Figure S2A). We subsequently performed complementation experiments in $V$. dahliae to confirm the importance of the C-terminus for activation of Ve1-mediated immunity. To this end, we expressed VdAve14126-134 driven by the native VdAve1 promoter in a VdAve1 deletion mutant (V. dahliae JR2 2 VdAve1; de Jonge et al., 2012) and inoculated Ve1 tomato plants with the complemented strains. Plants that were inoculated with three independent $V$. dahliae strains expressing VdAve1 ${ }^{\Delta 126-134}$ (VdAve1 ${ }^{\Delta 126-134} \# 1$, VdAve14126-134 \#2 and VdAve1 ${ }^{\Delta 126-134} \# 3$ ) showed a similar disease phenotype as Ve1 plants inoculated with the $V$. dahliae JR2 $\Delta V d A v e 1$ strain, whereas plants inoculated with wild-type $V$. dahliae strain JR2 and the VdAve1 complementation strain resembled mockinoculated plants (Figure 3C, D). Collectively, these results demonstrate that the Cterminal nine amino acids of VdAve1 are required to activate Ve1-mediated immunity.

Since the C-terminal nine amino acids appear to be essential for VdAve1 recognition, and the bacterial homolog XacPNP that is significantly divergent in this region (Figure 1A) is not recognized by Ve1 (Figure 1B, C), C-terminal nine-aminoacid swaps between VdAve1 and XacPNP were performed. An expression construct encoding a chimeric VdAve1 protein was engineered in which the C-terminal nine amino acids of VdAve1 were replaced by those of XacPNP (Vd_XacPNPc 9AA; Figure 4A). As expected, similar to XacPNP, co-expression of Vd_XacPNPc9AA with Ve1 in tobacco failed to induce an $\mathrm{HR}$, as only minimal necrotic spots were observed (Figure 4A, B). Conversely, a construct encoding another chimeric VdAve1 protein was generated in which the last nine amino acids of XacPNP were replaced by those of VdAve1 (Xac_VdAve1 ${ }^{\text {9AA; }}$ Figure 4A). Co-expression of Xac_VdAve1 ${ }^{\text {9AA }}$ with Ve1 in tobacco induced a relatively strong HR when compared with XacPNP or Vd_XacPNPc ${ }^{9 A A}$-induced $\mathrm{HR}$, although full necrosis was not observed in the infiltrated leaf area (Figure 4A, B). Immunoblotting confirmed that the GFP-tagged Vd_XacPNPc 9AA and Xac_VdAve1c ${ }^{9 A A}$ proteins were present in planta (Figure S2B). 

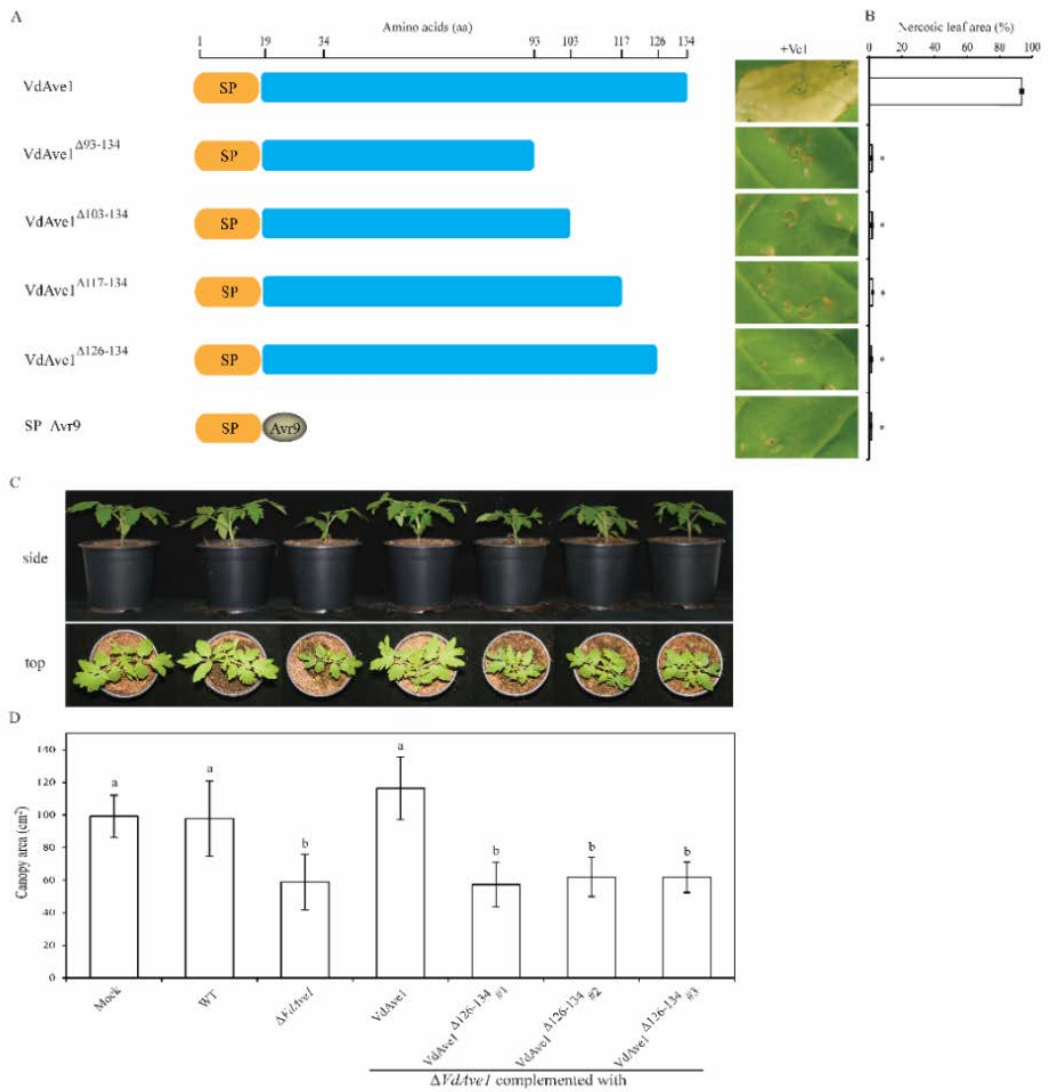

Figure 3. The C-terminal nine amino acids of VdAve1 are required to establish Ve1-mediated immunity. (A) VdAve1 C-terminal truncations result in loss of recognition by Ve1. Constructs encoding four VdAve1 truncations that lack the C-terminal 42 (VdAve1 $\left.{ }^{\Delta 93-134}\right), 32$ (VdAve1 $^{\Delta 103-134}$ ), 18 (VdAve1 ${ }^{\Delta 117-}$ ${ }^{134}$ ) and 9 (VdAve1 $\left.{ }^{\Delta 126-134}\right)$ amino acids of VdAve1 co-infiltration with Ve1 were assayed respectively, and the occurrence of necrosis was recorded at $5 \mathrm{dpi}$. Constructs VdAve1 and SP_Avr9 were used as a positive and negative control, respectively. The feature includes a predicated signal peptide (SP) involved in effector secretion. (B) Quantification of necrosis resulting from recognition of VdAve1 Cterminal truncations by Ve1 at 5 dpi. The graph shows the average percentage of necrotic leaf area of infiltration zones at $5 \mathrm{dpi}(\mathrm{n}>5)$. Data are presented as mean with standard deviations. Asterisks indicate statistically significant differences when compared with wild-type VdAve1-indcued necrosis $(P$ $<0.05$ ). (C) Complementation assays in Verticillium dahliae show that the VdAve1 C-terminal nine amino acids are required to activate Ve1-mediated immunity in tomato. Three independent V. dahliae VdAve1 deletion $(\triangle V d A v e 1)$ strains expressing a construct encoding VdAve1 lacking the C-terminal nine amino acids (VdAve1 ${ }^{\Delta 126-134} \# 1$, VdAve1 $1^{\Delta 126-134} \# 2$ and VdAve1 126-134 \#3) escape recognition by Ve1 tomato compared with $V$. dahliae wild-type (WT) and genetic complementation strains (VdAve1) evidenced by stunted Ve1 plants at 14 days post Verticillium inoculation. (D) Average canopy area of 8 Ve 1 tomato plants inoculated with different $V$. dahliae strains or mock-inoculation. Different letters indicate statistically significant differences $(P<0.05)$. The data shown are representative of three independent experiments. 


\section{Chapter 3}

\section{1}

VdAve1

XacPNP

Vd_XacP'N ${ }^{\mathrm{E}}{ }^{\mathrm{E} A \mathrm{AA}}$

Xac_VdAvel $1^{\text {c } 9 A . A}$

SP VdAvel ${ }^{\text {c.AA }}$

Synthesized Peptide

SP_GFP_VdAvel ${ }^{\text {9AAA }}$

SP_GFP

SP_Avr9_VdAvel ${ }^{\mathrm{c} 9 \mathrm{AA}}$

SP_Avr?
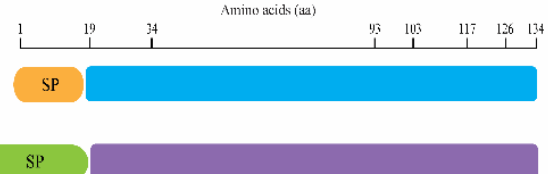

SP

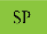

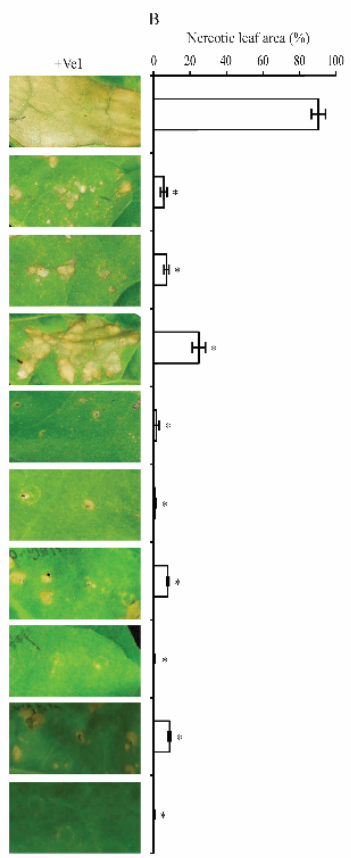

Figure 4. (A) The C-terminal nine amino acids of VdAve1 are critical but not sufficient for Ve1-mediated recognition in tobacco. Constructs encoding full-length VdAve1 and XacPNP with their own signal peptides (SP) and two chimeras in which their C-terminal nine amino acids were swapped (Vd_XacPNPc 9AA and Xac_VdAve1 ${ }^{\mathrm{C}}{ }^{9 \mathrm{AA}}$ ) were assayed. In chimera Vd_XacPNPc 9AA the C-terminal nine amino acids of VdAve1 were replaced by those of XacPNP, while in chimera Xac_VdAve1 ${ }^{\text {c } 9 A A}$ the C-terminal nine amino acids of XacPNP were replaced by those of XacPNP. A construct encodes the C-terminal nine amino acids

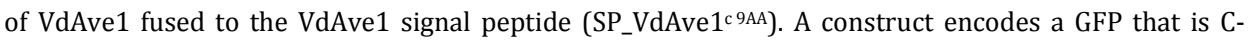
terminally fused to the C-terminal nine amino acids of VdAve1, and N-terminally fused to the VdAve1 signal peptide to establish extracellular secretion (SP_GFP_VdAve1 ${ }^{\text {9 } 9 A}$ ), while a construct encoding GFP fused with the VdAve1 signal peptide (SP_GFP) and construct SP_Avr9 were used as negative controls. Furthermore, a chemically synthetized peptide encompassing VdAve1 C-terminal nine amino acids peptide (AVNIEFRQI) was used. A construct VdAve1 was used as a positive control. The feature includes a predicated signal peptide (SP) involved in effector secretion. All the constructs were co-expressed with $V e 1$ in tobacco respectively, and the occurrence of necrosis was monitored at $5 \mathrm{dpi}$. (B) Quantification of necrosis resulting from recognition of VdAve 1 chimeras by Ve1 at $5 \mathrm{dpi}$. The graph shows the average percentage of necrotic leaf area of infiltration zones at $5 \mathrm{dpi}(\mathrm{n}>10)$. Data are presented as mean with standard deviations. Asterisks indicate statistically significant differences when compared with wildtype VdAve1-indcued necrosis $(P<0.05)$.

These results confirm that the C-terminal nine amino acids of VdAve1 are critical for recognition by $\mathrm{Ve} 1$, and furthermore suggest that these are sufficient for recognition. 
To determine whether the C-terminal nine amino acids are indeed sufficient to trigger Ve1-mediated recognition, we generated a construct encoding the Cterminal nine amino acids of VdAve1 fused to the VdAve1 signal peptide (SP_VdAve1 ${ }^{\text {9AA; }}$ Figure 4A). This construct was co-expressed with Ve1 in tobacco, but no necrosis was observed in the infiltrated leaf (Figure 4A, B). Additionally, infiltration of a chemically synthetized peptide encompassing the C-terminal nine amino acids of VdAve1 (peptide sequence: AVNIEFRQI) did not induce an HR in Ve1-expressing tobacco up to a concentration of $1 \mathrm{mg} / \mathrm{mL}$ (Figure 4A, B). However, possibly the nine amino acid peptide is not stable in the apoplast. In an attempt to overcome such complications, we generated two constructs in which the coding sequence of GFP or Avr9 was N-terminally fused to the VdAve1 signal peptide and C-terminally fused to the C-terminal nine amino acids of VdAve1 (SP_GFP_VdAve1c 9AA and SP_Avr9_VdAve1c 9AA; Figure 4A). As negative controls, two constructs in which the coding sequence of GFP or Avr9 was N-terminally fused to the VdAve1 signal peptide without the C-terminal nine amino acids of VdAve1 (SP_GFP and SP_Avr9; Figure 4A) were generated. All constructs were co-expressed with Ve1 in tobacco and HR development was monitored at 5 dpi. However, only slight necrosis was observed following infiltration of Ve1 with $S P_{-} G F P_{-} V d A v e 1^{c}$ 9AA or SP_Avr9_VdAve1 ${ }^{c 9 A A}$ in the infiltrated sector (Figure 4A, B), despite detection of SP_GFP_VdAve1 ${ }^{\text {9 } 9 A}$ protein in planta by immunoblotting (Figure S2A). These data reveal that the $\mathrm{C}$-terminal nine amino acids of VdAve1 are not sufficient to activate Ve1-mediated immunity.

To test whether longer stretches of the C-terminus of VdAve1 can be used to trigger Ve1-mediated recognition, we generated a construct encoding the $\mathrm{C}$ terminal 18 amino acids of VdAve1 fused to the VdAve1 signal peptide (SP_VdAve1 ${ }^{c}$ 18AA; Figure 5A). This construct was co-expressed with Ve1 in tobacco, but only slightly increased necrosis was observed in the infiltrated sector (Figure 5A, B). We further generated two constructs in which the coding sequence of GFP or Avr9 was $\mathrm{N}$-terminally fused to the VdAve1 signal peptide and C-terminally fused to the Cterminal 18 amino acids of VdAve1 (SP_GFP_VdAve1 ${ }^{\text {c18AA }}$ and SP_Avr9_VdAve1c18AA; Figure 5A). Co-infiltrations of Ve1 with $S P_{-} G F P$ or $S P_{-} A v r 9$ were used as negative controls. Co-expression of Ve1 with SP_GFP_VdAve1 ${ }^{\text {c18AA }}$ or SP_Avr9_VdAve1 ${ }^{c 18 A A}$ in tobacco induced similar necrosis formation as SP_VdAve1 ${ }^{\text {c } 18 \mathrm{AA}}$ protein at $5 \mathrm{dpi}$ 

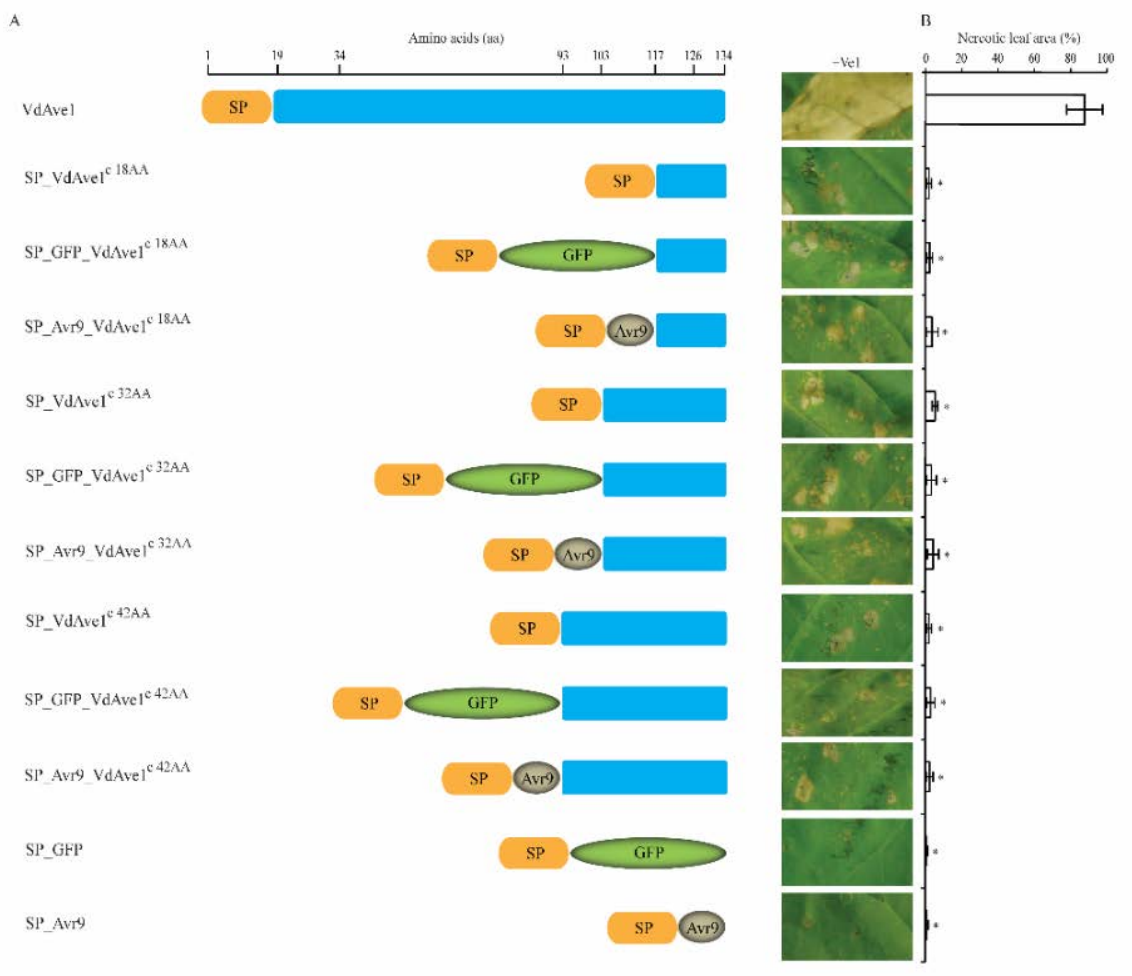

Figure 5. VdAve1 C-terminus is not sufficient for VdAve1 recognition by Ve1. (A) Occurrence of the necrosis in tobacco upon co-expression of VdAve1 C-terminal chimeras with Ve1. Three constructs encoding the VdAve1 signal peptide (SP) fused to the VdAve1 C-terminal 18-amino-acid (SP_VdAve1c ${ }^{18 \mathrm{AA}}$ ), or the GFP and VdAve1 C-terminal 18-amino-acid (SP_GFP_VdAve1 ${ }^{\mathrm{c} 18 \mathrm{AA}}$ ) , or the mature Avr9 and VdAve1 C-terminal 18-amino-acid (SP_Avr9_ VdAve1 ${ }^{\mathrm{c}}{ }^{18 \mathrm{AA}}$ ) were generated. Furthermore, constructs encoding the extended VdAve1 C-termini (SP_VdAve1 ${ }^{\text {c } 32 \mathrm{AA}}$ and SP_VdAve1 ${ }^{\mathrm{c}}{ }^{42 \mathrm{AA}}$ ), or N-terminally GFPtagged VdAve1 C-terminal extensions (SP_GFP_VdAve1 ${ }^{\text {c32AA }}$ and SP_GFP_VdAve1c ${ }^{42 A A}$ ), or N-terminally Avr9-fused VdAve1 C-terminal extensions (SP_Avr9_VdAve1c 32AA and SP_Avr9_VdAve1c 42AA) were assayed too. Constructs VdAve1, SP_GFP and SP_Avr9 were used as controls. The feature includes a predicated signal peptide (SP) involved in effector secretion. The constructs were co-expressed with Ve1 in tobacco respectively, and the necrosis occurrence was recorded at 5 dpi. (B) Quantification of necrosis resulting from recognition of VdAve1 C-terminal chimeras by Ve1 at 5 dpi. The graph shows the average percentage of necrotic leaf area of infiltration zones at 5 dpi $(n>10)$. Data are presented as mean with standard deviations. Asterisks indicate statistically significant differences when compared with wild-type VdAve1-indcued necrosis $(P<0.05)$.

(Figure 5A, B). Immunoblotting confirmed that the chimeric SP_GFP_VdAve1c18AA protein was present in planta (Figure S3). Subsequent analyses showed that also the C-terminal 32 amino acids and the C-terminal 42 amino acids of VdAve1 induce signs of a weak HR at 5 dpi (Figure 5A, B), although their presence was verified by 
immunoblotting (Figure S3). Taken together, these results reveal that the Cterminus of VdAve1 is required, but not sufficient, to activate Ve1-mediated recognition.

The N-terminus of VdAve1 is required, but not sufficient for Ve1-mediated recognition

We previously observed that affinity tags that were C-terminally fused to VdAve1 compromised recognition by $\mathrm{Ve} 1$, resulting in the finding that the C-terminus of VdAve1 is required for recognition. Accordingly, when we fused various tags (GFP, HA, Myc and Avr9) to the N-terminus of mature VdAve1, we similarly observed significantly compromised Ve1-mediated recognition upon use of the GFP (VdAve1_n GFP), or Myc (VdAve1_n Myc), or Avr9 (VdAve1_n Avr9) tag (Figure 2B, C), although N-terminal tagging of VdAve1 with HA (VdAve1_n HA) did not significantly affect recognition by Ve1 (Figure 2B, C). Immunoblotting confirmed that the N-terminally GFP-tagged VdAve1 protein was stably produced in planta (Figure S2A). These data suggest that, besides the VdAve1 C-terminus, also the Nterminus of VdAve1 is involved in recognition by Ve1.

To further investigate the importance of the N-terminus of VdAve1 in Ve1mediated recognition, recognition of VdAve1 upon deletion of the N-terminal 16 amino acids (from $\mathrm{Asp}^{19}$ to $\mathrm{Ala}^{34}$; VdAve1 ${ }^{\Delta 19-34}$ ) by Ve1 was tested in tobacco. Indeed, deletion of the $16 \mathrm{~N}$-terminal amino acids of the mature VdAve 1 abolished recognition by Ve1 (Figure 6A, B). Furthermore, we engineered two constructs in which the coding sequence of GFP or Avr9 was N-terminally fused to the VdAve1 signal peptide and C-terminally fused to the mature VdAve1 lacking the N-terminal 16-amino-acid of mature VdAve1 (SP_GFP_VdAve1 ${ }^{\Delta 19-34}$ and SP_Avr9_VdAve1 ${ }^{\text {19-34; }}$ Figure 6A). Co-expression of Ve1 with $S P_{-} G F P_{-} V d A v e 1^{\Delta 19-34}$ or SP_Avr9_VdAve1 $1^{\Delta 19-34}$ in tobacco did not result in Ve1-mediated recognition (Figure 6A, B), although production of SP_GFP_VdAve1 ${ }^{\Delta 19-34}$ was confirmed by immunoblotting (Figure S4). To further confirm the involvement of the VdAve1 N-terminus in Ve1-mediated recognition, we introduced the coding sequence VdAve $1^{\Delta 19-34}$ under the control of the native VdAve1 promoter into V. dahliae JR2 $\Delta V d A v e 1$ (de Jonge et al., 2012) and performed disease assays on $V e 1$ tomato plants. These assays showed that, similar to $V$. dahliae JR2 $\Delta V d A v e 1$, also complementation strains expressing VdAve1 $1^{\Delta 19-34}$ 
caused clear Verticillium wilt symptoms on Ve1 tomato plants, while wild-type $V$. dahliae strain JR2 and the complementation strain expressing VdAve1 caused no disease symptoms on tomato plants expressing Ve1 (Figure 6C, D). These results reveal that the $\mathrm{N}$-terminal 16 amino acids of mature VdAve 1 are required to trigger Ve1-mediated immunity.
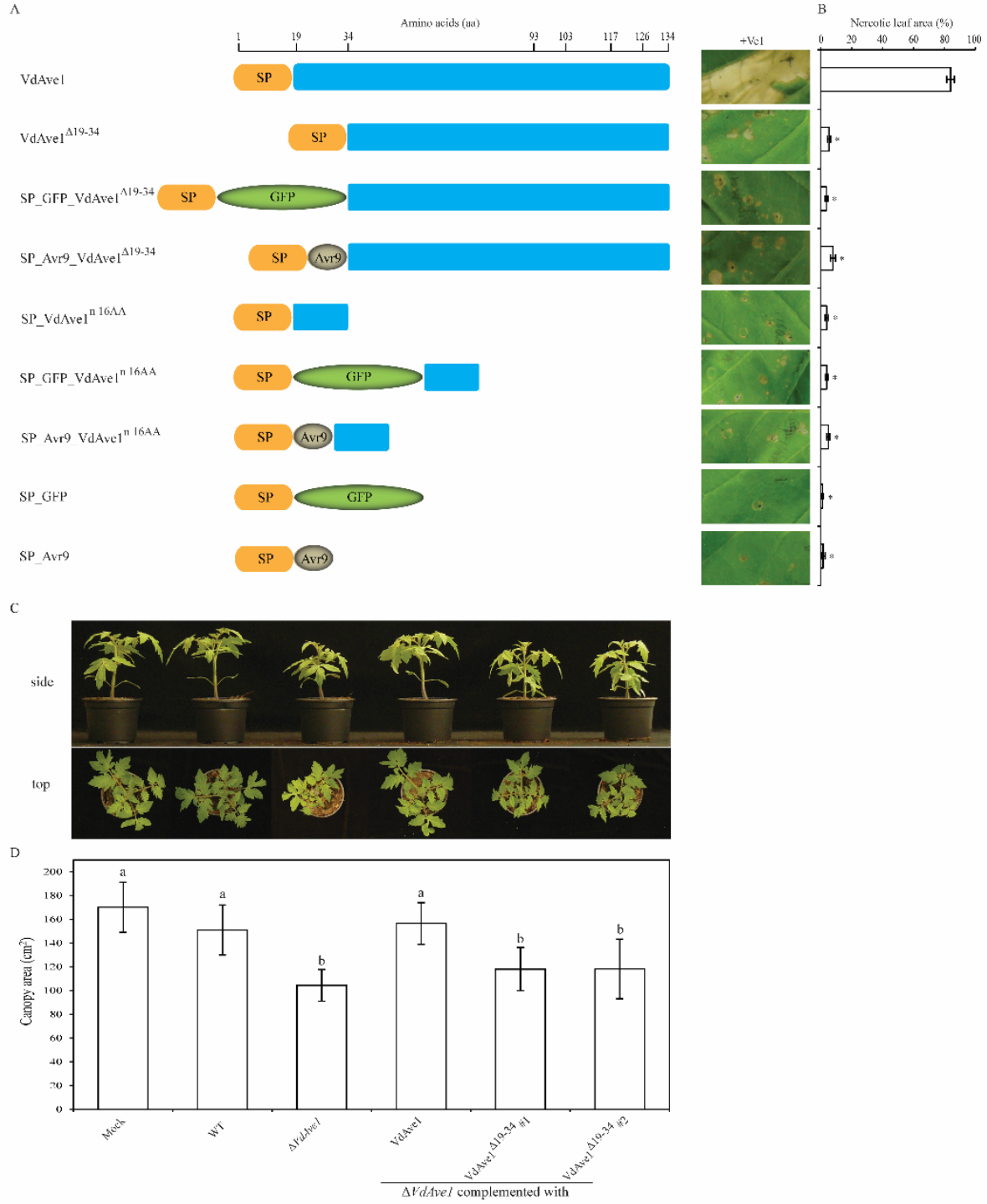
Figure 6. (A) The N-terminal sixteen amino acids of mature VdAve1 are required but not sufficient for Ve1-mediated recognition in tobacco. Occurrence of the necrosis in N. tabacum upon co-expression of Nterminal chimeras with Ve1. Constructs encoding a VdAve1 truncation that lacks the N-terminal 16amino-acid of mature VdAve1 (VdAve1 ${ }^{\Delta 19-34}$ ), and the corresponding N-terminal GFP or Avr9 fusion (SP_GFP_VdAve1 $1^{\Delta 19-34}$ and SP_Avr9_VdAve1 ${ }^{\Delta 19-34}$ ) were used. Furthermore, constructs encoding the VdAve1 signal peptide (SP) fused to the N-terminal 16-amino-acid of mature VdAve1 (SP_VdAve1 ${ }^{\text {n } 16 A A}$ ), or the GFP and the N-terminal 16-amino-acid of mature VdAve1 (SP_GFP_VdAve1 ${ }^{\mathrm{n} 16 \mathrm{AA}}$ ), or the mature Avr9 and VdAve1 C-terminal 16-amino-acid (SP_GFP_VdAve1 ${ }^{\text {n16AA }}$ ) were assayed. Constructs VdAve1, SP_GFP and SP_Avr9 were used as controls. The constructs include a predicted signal peptide (SP) to direct effector secretion. The constructs were co-agroinfiltrated with Ve1 in tobacco respectively, and the necrosis occurrence was monitored at 5 dpi. (B) Quantification of necrosis resulting from recognition of VdAve1 N-terminal chimeras by Ve1 at $5 \mathrm{dpi}$. The graph shows the average percentage of necrotic leaf area of infiltration zones at 5 dpi $(n>10)$. Data are presented as mean with standard deviations. Asterisks indicate statistically significant differences when compared with wild-type VdAve1-indcued necrosis $(P<0.05)$. (C) Complementation assays in $V$. dahliae show that the $\mathrm{N}$-terminal sixteen amino acids of mature VdAve1 protein are required to activate Ve1-mediated immunity in tomato. Two independent $V$. dahliae VdAve1 deletion ( $\triangle V$ VAve1) strains expressing a construct encoding VdAve1 lacking the N-terminal sixteen amino acids of mature VdAve1(VdAve1 ${ }^{\Delta 19-34} \# 1$ and VdAve1 ${ }^{\Delta 19-34}$ \#2) escape recognition by Ve1 tomato compared with $V$. dahliae wild-type (WT) and genetic complementation strains (VdAve1) evidenced by stunted Ve1 plants at 14 days post Verticillium inoculation. (D) Average canopy area of 8 Ve1 tomato plants inoculated with different $V$. dahliae strains or mock-inoculation Different letters indicate statistically significant differences $(P<0.05)$. The data shown are representative of three independent assays.

To examine whether that the N-terminal 16 amino acids are sufficient to trigger Ve1-mediated recognition, we engineered a construct encoding the $\mathrm{N}$ terminal 16-amino-acid of mature VdAve1 fused to the VdAve1 signal peptide

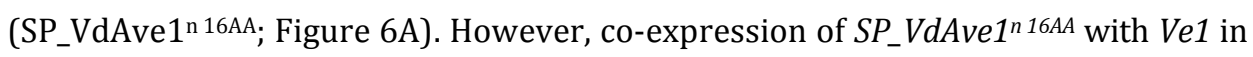
tobacco was not able to induce an HR at 5 dpi (Figure 6A, B). Furthermore, two constructs were designed in which the coding sequence of GFP or Avr9 was Nterminally fused to the VdAve1 signal peptide and C-terminally tagged to the $\mathrm{N}$ terminal 16-amino-acid of mature VdAve1 (SP_GFP_VdAve1 ${ }^{\mathrm{n}}$ 16AA and SP_Avr9_VdAve1n 16AA; Figure 6A). Co-expression of Ve1 with SP_GFP_VdAve1 ${ }^{\text {n } 16 A A}$ or SP_Avr9_VdAve1 ${ }^{n} 16 \mathrm{AA}$ in tobacco failed to induce necrosis in tobacco (Figure 6A, B), although immunoblotting showed that the SP_GFP_VdAve $1^{\text {n } 16 \mathrm{AA}}$ was present in planta (Figure S4). Collectively, these results demonstrate that the N-terminal 16 amino acids of mature VdAve1 are required, but not sufficient, to establish Ve1mediated immunity. 


\section{Structural modelling reveals co-localization of VdAve1 C- and N-termini on a surface-exposed patch}

In an attempt to gain a better insight in VdAve1 recognition by Ve1, a threedimensional structural model of VdAve1 was generated. Structural comparison with the protein databank (RCSB PDB) (Rose et al., 2013) revealed the maize protein EXPB1 (PDB ID: 2HCZ) (Yennawar et al., 2006) as a potential structural analogue (TM-Score 0.84 ) of VdAve1. The VdAve1 structural model shows that the C-terminal nine amino acids (Figure 7; shown in red) are exposed at the surface of the mature VdAve1 protein. The model also predicts that the N-terminus (Figure 7; shown in orange) congregates with the C-terminus on the exposed surface of the mature VdAve1 protein (Figure 7), which suggests that Ve1 may recognize a surface-exposed patch of the VdAve1 protein that includes both the $\mathrm{N}$ - and Ctermini. Taken together, we conclude that a surface-exposed patch of the VdAve1 protein that is composed of co-localized $\mathrm{N}$ - and $\mathrm{C}$-termini is recognized by the tomato cell-surface immune receptor Ve1.

\section{DISCUSSION}

We have previously shown that the $V$. dahliae effector VdAve1 is recognized by the tomato cell-surface immune receptor Ve1 (de Jonge et al., 2012), and we have identified several homologs of VdAve1 that are differentially recognized by Ve1 (Figure 1B, C). In this study, we reveal that a surface-exposed patch of the VdAve1 protein that is composed of co-localized $\mathrm{N}$ - and C-termini of $V$. dahliae effector VdAve1 is recognized by tomato immune receptor Ve1. Our analyses revealed that the C-terminus as well as the N-terminus individually are required, but not sufficient, to activate Ve1-mediated immunity.

Plant cell surface-localized PRRs are often activated upon recognition of short peptide sequences on the surface of their ligands, such as flg22 or flgII-28 that are derived from flagellin (Felix et al., 1999; Cai et al., 2011; Clarke et al., 2013), the pentapeptide TKLGE derived from EIX (Rotblat et al., 2002), the csp22 peptide derived from the bacterial cold shock proteins (Felix and Boller, 2003), elf18 or EFa50 derived from EF-Tu (Kunze et al., 2004; Furukawa et al., 2014), and the nlp20 peptide found in most NLPs (Böhm et al., 2014; Oome et al., 2014). In our study, we attempted to identify such motif in VdAve1 for recognition by the tomato 
A
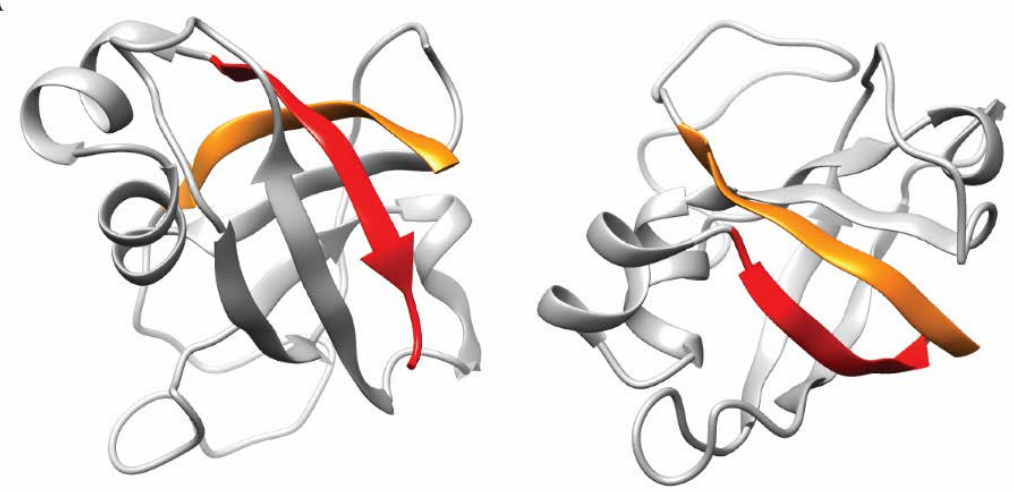

B
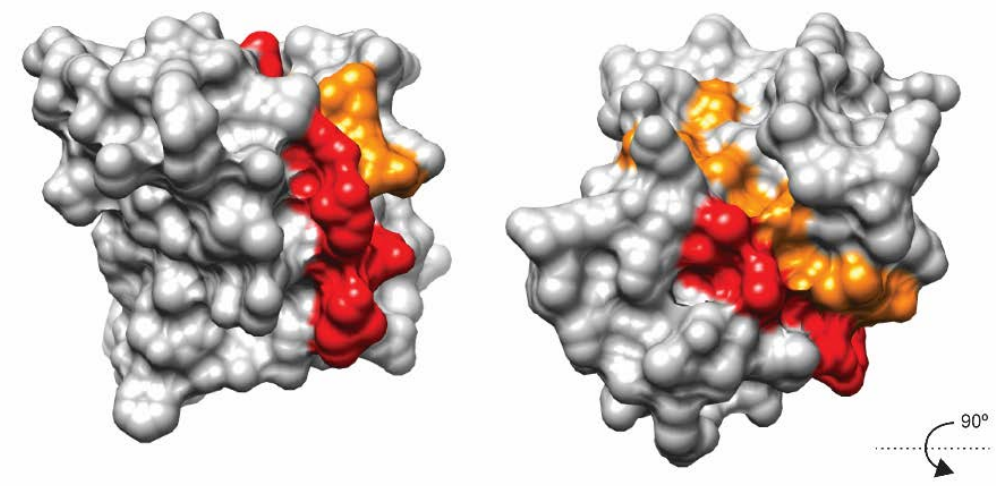

Figure 7. Three-dimensional structural model of the VdAve1 protein. The VdAve1 structure was predicted using I-TASSER (Zhang, 2008). The inferred VdAve1 structure is of high quality indicated by a confidence score (C-Score) of 1.22, and displayed as a ribbon (A), and surface (B) model from the side (left) and the top (right). The C-terminal nine amino acids sequence of VdAve1 is indicated in red, while the $\mathrm{N}$-terminal eight amino acids sequence is indicated in orange.

cell-surface immune receptor Ve1. Clearly, our results suggest that the colocalization of the two termini of the primary amino acid sequence, rather than a contiguous stretch of amino acids composes the recognition motif. Our efforts to identify an artificial minimal peptide that can be recognized by tomato Ve1 by generating chimeric peptides consisting of the $\mathrm{N}$-terminal sixteen amino acids in combination with various C-terminal peptides, and fused to various tags, were fruitless (data not shown). This suggests that also the folding of the recognized sequence patch, and the spatial orientation of the two protein termini, is important for the activation of Ve1-meidated immunity.

Although several minimal motifs have been identified in ligands of cell surface 


\section{Chapter 3}

receptors, similar examples for intracellular NLR (nucleotide-binding domain leucine-rich repeat) immune receptors have not been reported. Moreover, it has suggested that simultaneous recognition of multiple epitopes within a single effector is required for NLR activation. For example, distinct regions of the Pseudomonas syringae effector AvrRps4 are required for the activation of the intracellular NLR receptors PRS4/RRS1-mediated immunity (Sohn et al., 2009, 2012). Similarly, multiple contact points are likely required for recognition of the flax-rust effectors AvrL567 and AvrM by the corresponding NLR receptors in flax (Wang et al., 2007; Ve et al., 2013). Likewise, multiple residues at separate locations on the surface of the Hyaloperonospora arabidopsidis effector ATR1 are required for recognition by the Arabidopsis NLR receptor RPP1 (Chou et al., 2011; Goritschnig et al., 2016). In contrast to these findings, our data suggest that, although physically separated on the primary amino acid chain, recognition converges at a single surface-exposed patch of the VdAve1 protein.

Thus far, we have failed to show a direct physical interaction between VdAve1 and Ve1. Functional analysis of the tomato immune receptor Ve1 through domain swaps with its non-functional homolog Ve2, and subsequent alanine scanning mutagenesis on the solvent exposed $\beta$-strand/ $\beta$-turn residues across the eLRR domain previously identified several regions of the Ve1 protein that are required for functionality (Fradin et al., 2014; Zhang et al., 2014). In these studies, Ve1 functionality was restricted to three consecutive eLRR regions, namely eLRR1eLRR8, eLRR20-eLRR23 and eLRR32-eLRR37, of which two regions eLRR1-eLRR8 and eLRR20-eLRR23 were proposed to contribute to ligand binding, while eLRR32eLRR37 was proposed to function in immune signalling activation (Zhang et al., 2014). Realistically, final confirmation of this model cannot be obtained through domain swaps, domain deletions, gene shuffling analyses and site-directed mutagenesis within the immune receptor or the recognized ligand, but will ultimately have to follow from structural analysis of receptor-ligand interactions, for instance through crystallography.

\section{MATERIALS AND METHODS}

\section{Plant materials and plant growth conditions}

Tobacco (Nicotiana tabacum cv. Petite Havana SR1) and 35S::Ve1 tomato (Solanum 
lycopersicum cv. MoneyMaker background; Fradin et al., 2009) plants were grown in the greenhouse (Unifarm, Wageningen, the Netherlands) at $21^{\circ} \mathrm{C} / 19^{\circ} \mathrm{C}$ during 16/8 hours day/night periods, respectively, with 70\% relative humidity and 100 $\mathrm{W} / \mathrm{m}^{-2}$ supplemental light when the light intensity dropped below $150 \mathrm{~W} / \mathrm{m}^{-2}$. After agroinfiltration, tobacco plants were grown in the climate room at $22^{\circ} \mathrm{C} / 19^{\circ} \mathrm{C}$ during 16-h/8-h day/night periods, respectively, with $70 \%$ relative humidity.

\section{Sequence alignments and phylogenetic analysis}

Signal peptide prediction was performed using the SignalP 4.1 server (http://www.cbs.dtu.dk/services/SignalP/; Petersen et al., 2011). Multiple sequence alignments were conducted by using the Cluastal $\mathrm{X}$ program (http://www.clustal.org/). Phylogenetic tree was constructed by using the neighbour-joining method in MEGA7 (http://www.megasoftware.net/). Bootstrap reassembling analysis based on 1000 replicates was used to assess the confidence values indicated at the individual nodes.

\section{Generation of binary expression vectors}

Construction of all binary expression vectors is described in Methods S1.

\section{Transient expression assays}

Overnight cultures of $A$. tumefaciens strain GV3101 containing expression constructs were harvested at OD600 of 0.8 to 1 by centrifugation and resuspended to a final OD of 2 in infiltration medium as described previously (Zhang et al., 2013). A. tumefaciens cultures containing constructs to express Ve1 and VdAve1, or VdAve1 homologs, or VdAve1 chimeras were mixed in a 1:1 ratio and infiltrated into leafs of five- to six-week-old tobacco plants. At five days post infiltration (dpi), infiltrated leaves were photographed, and necrosis was quantified by using ImageJ to measure the area of necrosis as percentage of the total infiltrated leaf area (Song et al., 2017).

\section{Protein extracts and immunoblotting}

For immunological detection of GFP-tagged VdAve1 homologs and VdAve1 chimeras, A. tumefaciens carrying the corresponding expression constructs was 


\section{Chapter 3}

infiltrated into mature tobacco leaves as described previously (Zhang et al., 2013). The co-immunoprecipitations and immunoblotting were performed as described previously (Zhang et al., 2014).

\section{Generation of complementation V. dahliae strains}

To generate VdAve1, VdAve1 $1^{\Delta 126-134}$ and VdAve1419-34 complementation constructs, DNA fragments containing the $\mathrm{PacI}$ and NotI restriction sites were amplified by PCR from the corresponding plasmids VdAve1, VdAve1 ${ }^{\Delta 126-134}$ and VdAve1 ${ }^{\Delta 19-34}$ using primers listed in Table S2, and cloned into the vector pFBT005 which ToxA promoter was replaced by the native VdAve1 promoter $(\sim 1.6 \mathrm{~kb})$ and contains a nourseothricin cassette, respectively. All the constructs were confirmed by DNA sequencing (Eurofins Genomics, Ebersberg, Germany), and subsequently transformed into A. tumefaciens strain AGL1 by electroporation. A. tumefaciensmediated transformation of $V$. dahliae strain JR2 $\Delta V d A v e 1$ (de Jonge et al., 2012) was performed as previously described (Santhanam, 2012). V. dahliae transformants were selected on potato dextrose agar (PDA; Oxoid, Basingstoke, UK) plates containing $50 \mu \mathrm{g} / \mathrm{mL}$ nourseothricin sulphate (Sigma-Aldrich Chemie BV, Zwijndrecht, the Netherlands). After five to seven days at room temperature, individual transformants were transferred to fresh PDA plates and incubated for 7 to 10 days. Genomic DNA was extracted from individual transformants and PCR was performed to test presence of the inserted nourseothricin cassette and presence of the inserted chimeric VdAve1 fragment.

\section{Disease assays}

$V$. dahliae was grown on PDA plates at $22{ }^{\circ} \mathrm{C}$, and conidia were collected from 7 - to 10- day-old V. dahliae cultures on PDA plates and washed with tap water. Disease assays on tomato plants were performed as previously described (Fradin et al., 2009). Briefly, 10-day-old tomato plants were uprooted, the roots were rinsed in water, dipped for $5 \mathrm{~min}$ in a suspension of $10^{6}$ conidiospores $/ \mathrm{mL}$ water while the roots of mock plants were dipped in tap water without conidiospores, and transplanted to fresh commercial potting soil (Horticoop, Bleiswijk, the Netherlands). Disease symptoms were scored up to 14 days post Verticillium inoculation, inoculated plants were photographed. The canopy area of 8 plants was 
measured with ImageJ software and a One-Way ANOVA was performed with IBM SPSS statistics software.

\section{Generation of the structural model of VdAve1}

The V. dahliae VdAve1 structure was predicted using I-TASSER v4.3 (Zhang, 2008) and rendered using UCSF Chimera v1.10.1 (Pettersen et al., 2004). Structural predictions with C-Scores $>-1.5$ are generally considered to have a correct fold (CScores are typically in the range of $[-5,2])$; Roy et al., 2010). The structural analog in the protein data bank (RCSB PDB) (Rose et al., 2013) was identified using the TMalign program which is part of the I-TASSER package. Analogous structures with TM-Scores $>0.5$ are considered to have a similar fold (TM-Scores in the range $[0,1]$ ) (Roy et al., 2010).

\section{ACKNOWLEDGEMENTS}

Y.S. acknowledges a PhD fellowship from the China Scholarship Council (CSC). B.P.H.J.T. and M.F.S are supported by a Vici and a Veni grant, respectively, of the Research Council for Earth and Life sciences (ALW) of the Netherlands Organization for Scientific Research (NWO). We thank Bert Essenstam and Pauline Sanderson at Unifarm for excellent plant care, and Sebastjan Radišek at the Institute of Hop Research and Brewing, Slovenia, for providing Verticillium strains. 


\section{REFERENCES}

Albert I, Böhm H, Albert M, Feiler CE, Imkampe J, Wallmeroth N, Brancato C, Raaymakers TM, Oome S, Zhang H, Krol E, Grefen C, Gust AA, Chai J, Hedrich R, Van den Ackerveken G, Nürnberger T (2015) An RLP23-SOBIR1-BAK1 complex mediates NLP-triggered immunity. Nat Plants 1: 15140.

Böhm H, Albert I, Oome S, Raaymakers TM, Van den Ackerveken G, Nürnberger T (2014) A conserved peptide pattern from a qidespread microbial virulence factor triggers pattern-induced immunity in Arabidopsis. PLOS Pathog 10: e1004491.

Cai R, Lewis J, Yan S, Liu H, Clarke CR, Campanile F, Almeida NF, Studholme DJ, Lindeberg M, Schneider D (2011) The plant pathogen Pseudomonas syringae pv. tomato is genetically monomorphic and under strong selection to evade tomato immunity. PLOS Pathog 7: e1002130.

Ceroni A, Passerini A, Vullo A, Frasconi P (2006) DISULFIND: a disulfide bonding state and cysteine connectivity prediction server. Nucleic Acids Res 34: W177-181.

Chou S, Krasileva KV, Holton JM, Steinbrenner AD, Alber T, Staskawicz BJ (2011) Hyaloperonospora arabidopsidis ATR1 effector is a repeat protein with distributed recognition surfaces. Proc Natl Acad Sci USA 108: 13323-13328.

Clarke CR, Chinchilla D, Hind SR, Taguchi F, Miki R, Ichinose Y, Martin GB, Felix G, Vinatzer BA (2013) Allelic variation in two distinct Pseudomonas syringae flagellin epitopes modulates the strength of plant immune responses but not bacterial motility. New Phytol 200: 847-860.

Cook DE, Mesarich CH, Thomma BPHJ (2015) Understanding Plant Immunity as a Surveillance System to Detect Invasion. Annu Rev Phytopathol 53: 541-563.

de Jonge R, van Esse HP, Maruthachalam K, Bolton MD, Santhanam P, Saber MK, Zhang Z, Usami T, Lievens B, Subbarao KV, Thomma BPHJ (2012) Tomato immune receptor Ve1 recognizes effector of multiple fungal pathogens uncovered by genome and RNA sequencing. Proc Natl Acad Sci USA 109: 5110-5115.

Dodds PN, Rathjen JP (2010) Plant immunity: towards an integrated view of plant-pathogen interactions. Nat Rev Genet 11: 539-548.

Felix G, Boller T (2003) Molecular sensing of bacteria in plants the highly conserved rna-binding motif rnp-1 of bacterial cold shock proteins is recognized as an elicitor signal in tobacco. J Biol Chem 278: 6201-6208.

Felix G, Duran JD, Volko S, Boller T (1999) Plants have a sensitive perception system for the most conserved domain of bacterial flagellin. Plant J 18: 265-276.

Flor HH (1971) Current status of the gene-for-gene concept. Annu Rev Phytopathol 9: 275-296.

Fradin EF, Abd-El-Haliem A, Masini L, van den Berg GC, Joosten MH, Thomma BPHJ (2011) Interfamily transfer of tomato Ve1 mediates Verticillium resistance in Arabidopsis. Plant Physiol 156: 2255-2265.

Fradin EF, Thomma BPHJ (2006) Physiology and molecular aspects of Verticillium wilt diseases caused by V. dahliae and V. albo-atrum. Mol Plant Pathol 7: 71-86.

Fradin EF, Zhang Z, Ayala JCJ, Castroverde CD, Nazar RN, Robb J, Liu C-M, Thomma BPHJ (2009) Genetic dissection of Verticillium wilt resistance mediated by tomato Ve1. Plant Physiol 150: 320332.

Fradin EF, Zhang Z, Rovenich H, Song Y, Liebrand TW, Masini L, van den Berg GC, Joosten MH, Thomma BPHJ (2014) Functional analysis of the tomato immune receptor Ve1 through domain swaps with its non-functional homolog Ve2. PLOS ONE 9: e88208. 
Furukawa T, Inagaki H, Takai R, Hirai H, Che F-S (2014) Two distinct EF-Tu epitopes induce immune responses in rice and Arabidopsis. Mol Plant-Microbe Interact 27: 113-124.

Goritschnig S, Steinbrenner AD, Grunwald DJ, Staskawicz BJ (2016) Structurally distinct Arabidopsis thaliana NLR immune receptors recognize tandem WY domains of an oomycete effector. New Phytol 210: 984-996.

Inderbitzin P, Bostock RM, Davis RM, Usami T, Platt HW, Subbarao KV (2011) Phylogenetics and taxonomy of the fungal vascular wilt pathogen Verticillium, with the descriptions of five new species. PLOS ONE 6: e28341.

Isaac I (1953) A further comparative study of pathogenic isolates of Verticillium: V. nubilum Pethybr. and V. tricorpus sp. nov. Trans Br Mycol Soc 36: 180-195.

Jones JD, Dangl JL (2006) The plant immune system. Nature 444: 323-329.

Kawchuk LM, Hachey J, Lynch DR, Kulcsar F, van Rooijen G, Waterer DR, Robertson A, Kokko E, Byers R, Howard RJ (2001) Tomato Ve disease resistance genes encode cell surface-like receptors. Proc Natl Acad Sci USA 98: 6511-6515.

Kunze G, Zipfel C, Robatzek S, Niehaus K, Boller T, Felix G (2004) The N terminus of bacterial elongation factor Tu elicits innate immunity in Arabidopsis plants. Plant Cell 16: 3496-3507.

Oome S, Raaymakers TM, Cabral A, Samwel S, Böhm H, Albert I, Nürnberger T, Van den Ackerveken G (2014) Nep1-like proteins from three kingdoms of life act as a microbe-associated molecular pattern in Arabidopsis. Proc Natl Acad Sci USA 111: 16955-16960.

Petersen TN, Brunak S, von Heijne G, Nielsen H (2011) SignalP 4.0: discriminating signal peptides from transmembrane regions. Nat Methods 8: 785-786.

Pettersen EF, Goddard TD, Huang CC, Couch GS, Greenblatt DM, Meng EC, Ferrin TE (2004) UCSF Chimera - A visualization system for exploratory research and analysis. J Comput Chem 25: 16051612.

Pruitt RN, Schwessinger B, Joe A, Thomas N, Liu F, Albert M, Robinson MR, Chan LJG, Luu DD, Chen H (2015) The rice immune receptor XA21 recognizes a tyrosine-sulfated protein from a Gramnegative bacterium. Sci Adv 1: e1500245.

Ron M, Avni A (2004) The receptor for the fungal elicitor ethylene-inducing xylanase is a member of a resistance-like gene family in tomato. Plant Cell 16: 1604-1615.

Rose PW, Bi C, Bluhm WF, Christie CH, Dimitropoulos D, Dutta S, Green RK, Goodsell DS, Prlić A, Quesada M, Quinn GB, Ramos AG, Westbrook JD, Young J, Zardecki C, Berman HM, Bourne PE (2013) The RCSB Protein Data Bank: new resources for research and education. Nucleic Acids Res 41: D475-D482.

Rotblat B, Enshell-Seijffers D, Gershoni JM, Schuster S, Avni A (2002) Identification of an essential component of the elicitation active site of the EIX protein elicitor. Plant J 32: 1049-1055.

Roy A, Kucukural A, Zhang Y (2010) I-TASSER: a unified platform for automated protein structure and function prediction. Nat Protoc 5: 725-738.

Santhanam P (2012) Random insertional mutagenesis in fungal genomes to identify virulence factors. In Plant Fungal Pathogens (Melvin D. Bolton and Bart P.H.J. Thomma eds), pp. 509-517. New York: Springer.

Schaible L, Cannon OS, Waddoups V (1951) Inheritance of resistance to Verticillium wilt in a tomato cross. Phytopathology 41: 986-990

Sohn KH, Hughes RK, Piquerez SJ, Jones JD, Banfield MJ (2012) Distinct regions of the Pseudomonas syringae coiled-coil effector AvrRps4 are required for activation of immunity. Proc National Acad Sciences USA 109: 16371-16376. 


\section{Chapter 3}

Sohn KH, Zhang Y, Jones JD (2009) The Pseudomonas syringae effector protein, AvrRPS4, requires in planta processing and the KRVY domain to function. Plant J 57: 1079-1091.

Song Y, Zhang Z, Seidl MF, Majer A, Jakse J, Javornik B, Thomma BPHJ (2017) Broad taxonomic characterization of Verticillium wilt resistance genes reveals an ancient origin of the tomato Ve1 immune receptor. Mol Plant Pathol 18: 195-209.

Thomma BPHJ, Nurnberger T, Joosten MHAJ (2011) Of PAMPs and effectors: The blurred PTI-ETI dichotomy. Plant Cell 23: 4-15.

van Kan JA, van den Ackerveken G, de Wit PJGM (1991) Cloning and characterization of cDNA of avirulence gene avr9 of the fungal pathogen Cladosporium fulvum, causal agent of tomato leaf mold. Mol Plant-Microbe Interact 4: 52-59.

Ve T, Williams SJ, Catanzariti A-M, Rafiqi M, Rahman M, Ellis JG, Hardham AR, Jones DA, Anderson PA, Dodds PN (2013) Structures of the flax-rust effector AvrM reveal insights into the molecular basis of plant-cell entry and effector-triggered immunity. Proc Natl Acad Sci USA 110: 1759417599

Wang C-IA, Gunčar G, Forwood JK, Teh T, Catanzariti A-M, Lawrence GJ, Loughlin FE, Mackay JP, Schirra HJ, Anderson PA (2007) Crystal structures of flax rust avirulence proteins AvrL567-A and-D reveal details of the structural basis for flax disease resistance specificity. Plant Cell 19: 2898-2912.

Wang G, Fiers M, Ellendorff U, Wang Z, de Wit PJ, Angenent GC, Thomma BPHJ (2010) The diverse roles of extracellular leucine-rich repeat-containing receptor-like proteins in plants. Cri Rev Plant Sci 29: 285-299.

Wang L, Albert M, Einig E, Fürst U, Krust D, Felix G (2016) The pattern-recognition receptor CORE of Solanaceae detects bacterial cold-shock protein. Nat Plants 2: 16185.

Yennawar NH, Li LC, Dudzinski DM, Tabuchi A, Cosgrove DJ (2006) Crystal structure and activities of EXPB1 (Zea m 1), a beta-expansin and group-1 pollen allergen from maize. Proc Natl Acad Sci USA 103: 14664-14671.

Zhang Y (2008) I-TASSER server for protein 3D structure prediction. BMC Bioinformatics 9: 40.

Zhang Z, Fradin E, de Jonge R, van Esse HP, Smit P, Liu C-M, Thomma BPHJ (2013) Optimized agroinfiltration and virus-induced gene silencing to study Ve1-mediated Verticillium resistance in tobacco. Mol Plant-Microbe Interact 26: 182-190.

Zhang Z, Song Y, Liu C-M, Thomma BPHJ (2014) Mutational analysis of the Ve1 immune receptor that mediates Verticillium resistance in tomato. PLOS ONE 9: e99511.

Zipfel C, Kunze G, Chinchilla D, Caniard A, Jones JD, Boller T, Felix G (2006) Perception of the bacterial PAMP EF-Tu by the receptor EFR restricts Agrobacterium-mediated transformation. Cell 125: 749-760.

Zipfel C, Robatzek S, Navarro L, Oakeley EJ, Jones JD, Felix G, Boller T (2004) Bacterial disease resistance in Arabidopsis through flagellin perception. Nature 428: 764-767. 


\section{SUPPLEMENTAL INFORMATION}

Table S1. Verticillium strains analysed for presence of VdAve1 homologs.

\begin{tabular}{|c|c|c|c|c|}
\hline Species & Isolate & Original host & Origin & Ave1 \\
\hline \multirow[t]{43}{*}{ V. dahliae } & JKG2 & catalpa & Netherlands & - \\
\hline & CIG3-Vd & hop & Slovenia & - \\
\hline & JKG1 & potato & Netherlands & - \\
\hline & JKG8 & potato & Netherlands & - \\
\hline & A56 & potato & Slovenia & + \\
\hline & DJK & chrysanthemum & Netherlands & - \\
\hline & MH & chrysanthemum & Netherlands & - \\
\hline & Mint & $\operatorname{mint}$ & USA & - \\
\hline & T9 & cotton & USA & - \\
\hline & Vd1795 & sugar beet & USA & - \\
\hline & Vd850-4 & potato & USA & - \\
\hline & GAJ09 & hop & Slovenia & - \\
\hline & PDRENU & hop & Slovenia & - \\
\hline & CasD & hop & Slovenia & - \\
\hline & KresD & hop & Slovenia & - \\
\hline & MoD & hop & Slovenia & - \\
\hline & Oset & hop & Slovenia & - \\
\hline & 12099 & hop & U.K. & - \\
\hline & 12042 & hop & U.K. & - \\
\hline & PD335 & cabbage & unknown & - \\
\hline & PD584 & $\operatorname{mint}$ & unknown & - \\
\hline & V-176l & cotton & Netherlands & + \\
\hline & V-138l & cotton & Germany & - \\
\hline & PAPmb & pepper & Slovenia & - \\
\hline & PAP & pepper & Slovenia & + \\
\hline & Pap99 & pepper & Slovenia & + \\
\hline & Pap2008 & pepper & Slovenia & + \\
\hline & $2009-605$ & pepper & Ukrain & + \\
\hline & Vd52 & pepper & Austria & + \\
\hline & V4 & cotton & Spain & + \\
\hline & V117 & cotton & Spain & - \\
\hline & V991 & cotton & China & - \\
\hline & BP2 & cotton & China & - \\
\hline & CQ2 & cotton & China & - \\
\hline & $1 \mathrm{~cd} 3-2$ & cotton & China & - \\
\hline & 4TM6-15 & cotton & China & - \\
\hline & $1 \mathrm{hn}-1$ & cotton & China & - \\
\hline & CFA3 & sunflower & China & - \\
\hline & GRN1 & sunflower & China & - \\
\hline & GYA2 & sunflower & China & - \\
\hline & HnA4 & sunflower & China & - \\
\hline & SX4 & sunflower & China & - \\
\hline & WYA3 & sunflower & China & - \\
\hline
\end{tabular}


Chapter 3

\begin{tabular}{|c|c|c|c|c|}
\hline Species & Isolate & Original host & Origin & Ave1 \\
\hline \multirow[t]{10}{*}{ V. dahliae } & $\mathrm{HeA} 4$ & sunflower & China & - \\
\hline & 12 & sunflower & China & - \\
\hline & 77 & sunflower & China & - \\
\hline & 89 & sunflower & China & - \\
\hline & VPRI 42056 & tomato & Australia & - \\
\hline & VPRI 42057 & tomato & Australia & - \\
\hline & VPRI 42058 & tomato & Australia & - \\
\hline & VPRI 42079 & tomato & Australia & - \\
\hline & VPRI 42080 & tomato & Australia & - \\
\hline & VPRI 42081 & tomato & Australia & - \\
\hline \multirow[t]{36}{*}{ V. nonalfalfae } & P10 & hop & Germany & - \\
\hline & $\mathrm{P} 114 / 1$ & hop & Germany & - \\
\hline & P34/1 & hop & Germany & - \\
\hline & P15 & hop & Germany & - \\
\hline & P55 & hop & Germany & - \\
\hline & P83 & hop & Germany & - \\
\hline & $6 / 99$ & hop & Germany & - \\
\hline & $14 / 93$ & hop & Germany & - \\
\hline & $15 / 98$ & hop & Germany & - \\
\hline & $\mathrm{P} 84 / 2$ & hop & Germany & - \\
\hline & $16 / 00$ & hop & Germany & - \\
\hline & $\mathrm{T} 2$ & hop & Slovenia & - \\
\hline & TABOR6 & hop & Slovenia & - \\
\hline & Ledina09-V.aa & hop & Slovenia & + \\
\hline & BIZ & hop & Slovenia & - \\
\hline & VranBis09 & hop & Slovenia & - \\
\hline & Sent4 & hop & Slovenia & - \\
\hline & M03 & hop & Slovenia & - \\
\hline & oCer & hop & Slovenia & - \\
\hline & zup & hop & Slovenia & - \\
\hline & $\operatorname{Rec} 91$ & hop & Slovenia & - \\
\hline & KRES98 & hop & Slovenia & - \\
\hline & Gajsek & hop & Slovenia & - \\
\hline & $1985 a$ & hop & U.K. & - \\
\hline & 11041 & hop & U.K. & - \\
\hline & 11055 & hop & U.K. & - \\
\hline & 11047 & hop & U.K. & - \\
\hline & 11097 & hop & U.K. & - \\
\hline & 11100 & hop & U.K. & - \\
\hline & 1974 & hop & U.K. & - \\
\hline & 298099 & hop & U.K. & - \\
\hline & 298100 & hop & U.K. & - \\
\hline & 298101 & hop & U.K. & - \\
\hline & 298102 & hop & U.K. & - \\
\hline & 11052 & hop & U.K. & - \\
\hline & 1953 & hop & U.K. & - \\
\hline
\end{tabular}


Recognition of Verticillium dahliae effector Ave1 by tomato Ve1

\begin{tabular}{|c|c|c|c|c|}
\hline Species & Isolate & Original host & Origin & Ave1 \\
\hline \multirow[t]{23}{*}{ V. nonalfalfae } & 298092 & hop & U.K. & - \\
\hline & 298095 & hop & U.K. & - \\
\hline & Sol & hop & Poland & + \\
\hline & CBS393.91 & hop & Belgium & - \\
\hline & kum & cucumber & Slovenia & - \\
\hline & Surf & surfinias & Slovenia & - \\
\hline & 11077 & Galinsoga ciliata & U.K. & - \\
\hline & 11081 & chrysanthemum & U.K. & + \\
\hline & CBS102.464 & cynara & Italija & - \\
\hline & CBS241.82 & catalpa & Italija & + \\
\hline & CBS454.51 & potato & U.K. & - \\
\hline & CBS682.88 & potato & Netherlands & - \\
\hline & 11066 & potato & U.K. & - \\
\hline & T179 & tomato & U.K. & + \\
\hline & CBS321.91 & tomato & Netherlands & - \\
\hline & AR01/067 & tomato & U.K. & - \\
\hline & AR0/140 & tomato & U.K. & - \\
\hline & AR01/JS1 & tomato & U.K. & - \\
\hline & PD83/53a & tomato & Netherlands & - \\
\hline & PD2000/4186a & tomato & Netherlands & + \\
\hline & VnaCBS385.91 & tomato & Netherlands & + \\
\hline & Vna5431 & tomato & unknown & + \\
\hline & Vna1 & unknown & Luxembourg & - \\
\hline \multirow[t]{9}{*}{ V. alfalfae } & Luc & alfalfa & U.K. & + \\
\hline & 41 & alfalfa & Canada & - \\
\hline & CBS392.91 & alfalfa & Netherlands & - \\
\hline & Va2 & unknown & Netherlands & - \\
\hline & 107 & alfalfa & USA & - \\
\hline & PD693 & potato & Iran & - \\
\hline & 314193 & potato & Australia & - \\
\hline & 340646 & potato & Spain & + \\
\hline & 11 & alfalfa & Slovenia & - \\
\hline \multirow[t]{4}{*}{ V. nubilum } & CBS456.51 & potato & UK & + \\
\hline & CBS457.51 & soil & UK & + \\
\hline & PD621 & mushroom compost & UK & + \\
\hline & PD702 & potato & UK & + \\
\hline \multirow[t]{2}{*}{ V. tricorpus } & CBS227.84 & potato & Netherlands & - \\
\hline & JKG20 & linden & Netherlands & - \\
\hline \multirow[t]{2}{*}{ V. longisporium } & CBS110218 & Brassica napus & Sweden & - \\
\hline & PD330 & cabbage & unknown & - \\
\hline
\end{tabular}




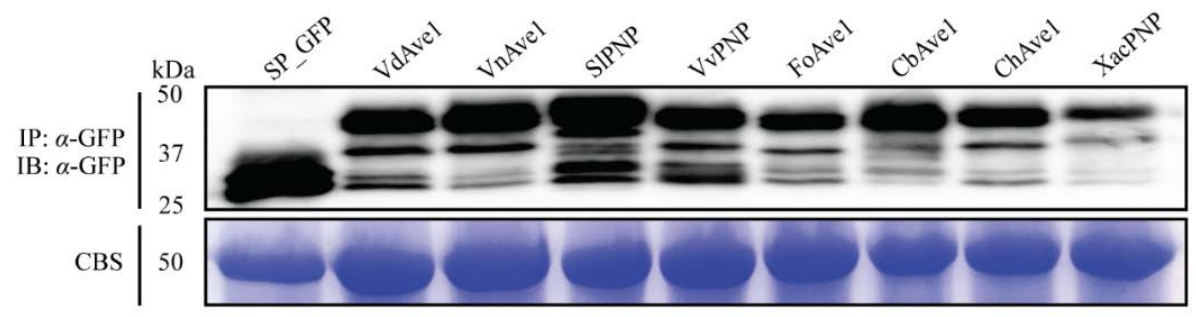

Figure S1. Stability of N-terminally GFP-tagged of mature VdAve1 homologs proteins in planta. Total protein extracts of transiently transformed leaf tissue were subjected to immunoprecipitation(IP) using $\alpha$-GFP affinity beads. Immunoprecipitation proteins were subjected to sodium dodecyl sulfatepolyacrylamide electrophoresis (SDS/PAGE) and immunoblotted (IB) using $\alpha$-GFP antibody. Coomassie blue staining (CBS) of the blot containing total protein extracts showed equal loading in each lane based on the 50-kDa RuBisCo (ribulose-1,5-bisphosphate carboxylase/oxygenase) band. GFP-tagged VdAve1 signal peptide was used as a control.

A

B
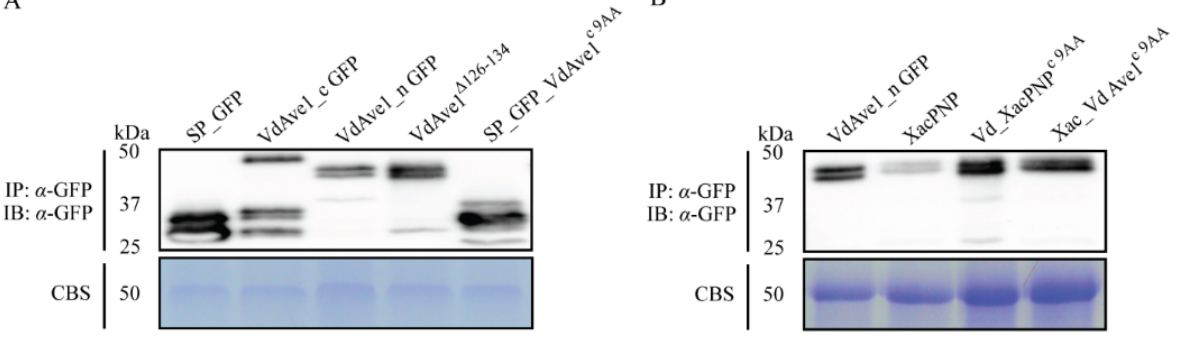

Figure S2. Presence of GFP-tagged VdAve1 chimeras proteins in planta. (A) VdAve1_c GFP, VdAve1_n GFP, SP_GFP_VdAve1 ${ }^{\text {c9AA }}$ and N-terminally GFP-tagged VdAve1 ${ }^{\Delta 126-134}$ proteins are detected in planta by immunoprecipitation (IP) using $\alpha$-GFP affinity beads, followed by immunoblotting (IB) using $\alpha$-GFP antibody. Coomassie blue staining (CBS) of the blot containing total protein extracts showed equal loading in each lane based on the 50-kDa RuBisCo (ribulose-1,5-bisphosphate carboxylase/oxygenase) band. GFP-tagged VdAve1 signal peptide was used as a control. (B) N-terminally GFP-tagged XacPNP, Vd_XacPNPc 9AA and XacP_VdAve1 ${ }^{\text {9 } 9 \mathrm{AA}}$ proteins are detected in planta by immunoprecipitation (IP) using $\alpha$-GFP affinity beads, followed by immunoblotting (IB) using $\alpha$-GFP antibody. Coomassie blue staining (CBS) of the blot containing total protein extracts showed equal loading in each lane based on the 50kDa RuBisCo (ribulose-1,5-bisphosphate carboxylase/oxygenase) band. GFP-tagged VdAve1 signal peptide and VdAve1_n GFP were used as controls. 


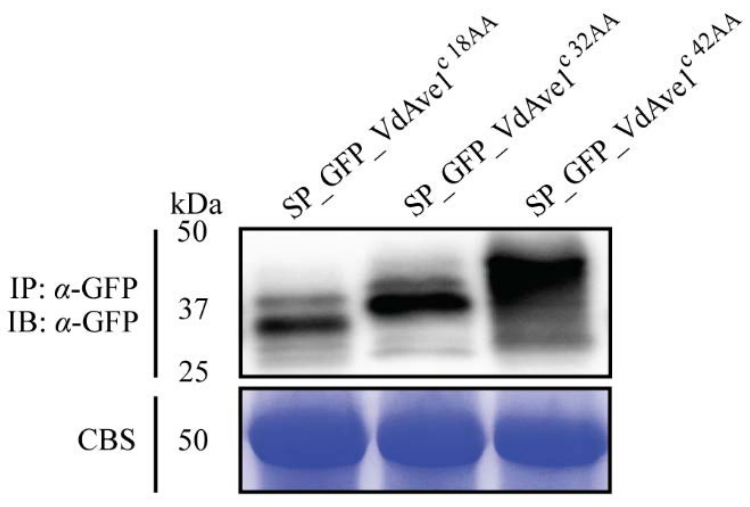

Figure S3. Stability of N-terminally GFP-tagged VdAve1 C-termini proteins in planta. SP_GFP_VdAve1 ${ }^{1}{ }^{18 A A}, S_{-}$SPFP_VdAve1 ${ }^{\text {c } 32 A A}$ and SP_GFP_VdAve1c ${ }^{42 A A}$ proteins are detected in planta by immunoprecipitation (IP) using $\alpha$-GFP affinity beads, followed by immunoblotting (IB) using $\alpha$-GFP antibody. Coomassie blue staining (CBS) of the blot containing total protein extracts showed equal loading in each lane based on the 50-kDa RuBisCo (ribulose-1,5-bisphosphate carboxylase/oxygenase) band.

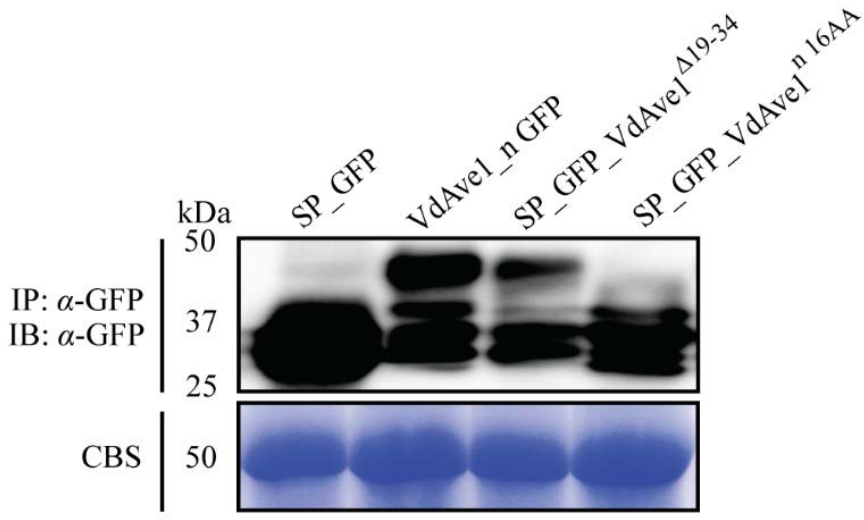

Figure S4. Presence of GFP-tagged VdAve1 N-terminal truncation proteins in planta. SP_GFP_VdAve $1^{\Delta 18-34}$ and SP_GFP_VdAve1 ${ }^{\mathrm{n}}$ 16AA proteins are detected in planta by immunoprecipitation (IP) using $\alpha$-GFP affinity beads, followed by immunoblotting (IB) using $\alpha$-GFP antibody. Coomassie blue staining (CBS) of the blot containing total protein extracts showed equal loading in each lane based on the 50-kDa RuBisCo (ribulose-1,5-bisphosphate carboxylase/oxygenase) band. GFP-tagged VdAve1 signal peptide and VdAve1_n GFP were used as controls. 
Chapter 3

Table S2. Primers used in this study.

\begin{tabular}{|c|c|c|}
\hline Primer name & Oligonucleotide sequence $\left(5^{\prime} \rightarrow 3^{\prime}\right)$ & Description $^{\mathrm{a}}$ \\
\hline VdAve1-F (attB1) & ggggacaagtttgtacaaaaaagcaggctATGAAGCTTTCTACGCTT & VdAve1 and VdAve1 chimeras \\
\hline VdAve1-R (attB2) & ggggaccactttgtacaagaaagctgggtTTATATCTGTCTAAATTC & VdAve1 and VdAve1 chimeras \\
\hline VnAve1-F (attB1) & ggggacaagtttgtacaaaaaagcaggctATGAAGCTTTCTACGCTT & VnAve1 \\
\hline VnAve1-R (attB2) & ggggaccactttgtacaagaaagctgggtTTATATCTGTTCAAACTC & VnAve1 \\
\hline SIPNP-R (attB2) & ggggaccactttgtacaagaaagctgggtTCAAATCTGGACATATTC & SIPNP \\
\hline VvPNP-R (attB2) & ggggaccactttgtacaagaaagctgggtTCAAATCTGTATGTACTC & VvPNP \\
\hline FoAve1-R (attB2) & ggggaccactttgtacaagaaagctgggtTCATCTTTGTACAAAATCG & FoAve1 \\
\hline CbAve1-R (attB2) & ggggaccactttgtacaagaaagctgggtCTATATCTGCATATACTC & CbAve1 \\
\hline ChAve1-R (attB2) & ggggaccactttgtacaagaaagctgggtTCAAATTTGTACGTACTC & ChAve1 \\
\hline XacPNP-R (attB2) & ggggaccactttgtacaagaaagctgggtTTAAATATTTGCCCAGGG & XacPNP \\
\hline VdAve1( $\Delta \mathrm{SC})-\mathrm{R}(\mathrm{attB} 2)$ & ggggaccactttgtacaagaaagctgggtcTATCTGTCTAAATTC & VdAve1 (C-terminal tag) \\
\hline VdAve1 ${ }^{\Delta 93-134-R}$ (attB2) & ggggaccactttgtacaagaaagctgggtTTACTTATGCCTCGTTCCCTT & VdAve1 $1^{\Delta 93-134}$ \\
\hline VdAve1 ${ }^{\Delta 103-134-R}$ (attB2) & ggggaccactttgtacaagaaagctgggtTTAAACAATGGCATCATATGAGT & VdAve1 $1^{\Delta 103-134}$ \\
\hline VdAve1 $1^{\Delta 117-134}-\mathrm{R}$ (attB2) & ggggaccactttgtacaagaaagctgggtTTACTTGTGTGCTGCTTTGGTAA & VdAve1 $1^{\Delta 117-134}$ \\
\hline VdAve1 ${ }^{\Delta 126-134-R}($ attB2) & ggggaccactttgtacaagaaagctgggtTTAAGCTCTGTCAACCACCCGCA & VdAve1 $1^{\Delta 126-134}$ \\
\hline GFP-VdAve1 ${ }^{\text {c } 9 A A-R}$ (attB2) & $\begin{array}{l}\text { ggggaccactttgtacaagaaagctgggtTTATATCTGTCTAAATTCGATGTT } \\
\text { GACCGCTTTGTATAGTTCATCCATGC }\end{array}$ & SP_GFP_VdAve1c9AA \\
\hline Avr9-VdAve1 ${ }^{\text {c9AA-R }}$ & $\begin{array}{l}\text { TTATATCTGTCTAAATTCGATGTTGACCGCCTTATGCCTCGTTCCCT } \\
\text { TCCACTGATTATGTACACATTGGAGCTTA }\end{array}$ & SP_Avr9_VdAve1 ${ }^{\text {c } 9 \text { AA }}$ \\
\hline GFP-VnAve1-F & GCATGGATGAACTATACAAACAATTAGGGACCGCATCC & VnAve1_n GFP \\
\hline GFP-VnAve1-R & GGATGCGGTCCCTAATTGTTTGTATAGTTCATCCATGC & VnAve1_n GFP \\
\hline GFP-SIPNP-F & GCATGGATGAACTATACAAAGATATTGGCACGGCTAC & SIPNP_n GFP \\
\hline GFP-SIPNP-R & GTAGCCGTGCCAATATCTTTGTATAGTTCATCCATGC & SIPNP_n GFP \\
\hline GFP-VvPNP-F & GCATGGATGAACTATACAAAGACATTGGCACTGCAAAC & VvPNP_n GFP \\
\hline GFP-VvPNP-R & GTTTGCAGTGCCAATGTCTTTGTATAGTTCATCCATGC & VvPNP_n GFP \\
\hline GFP-FoAve1-F & GCATGGATGAACTATACAAAGATATCGGAACTGCAAATATTC & FoAve1_n GFP \\
\hline GFP-FoAve1-R & GAATATTTGCAGTTCCGATATCTTTGTATAGTTCATCCATGC & FoAve1_n GFP \\
\hline GFP-CbAve1-F & GCATGGATGAACTATACAAAGACATCGGCACCGCCGTC & CbAve1_n GFP \\
\hline GFP-CbAve1-R & GACGGCGGTGCCGATGTCTTTGTATAGTTCATCCATGC & CbAve1_n GFP \\
\hline GFP-ChAve1-F & GCATGGATGAACTATACAAAGATATCGGAACAGCAGGC & ChAve1_n GFP \\
\hline GFP-ChAve1-R & GCCTGCTGTTCCGATATCTTTGTATAGTTCATCCATGC & ChAve1_n GFP \\
\hline GFP-XacPNP-F & GCATGGATGAACTATACAAAGACATCGGTACAATTAG & XacPNP_n GFP \\
\hline GFP-XacPNP-R & CTAATTGTACCGATGTCTTTGTATAGTTCATCCATGC & XacPNP_n GFP \\
\hline GFP-Vd_XacPNPc 9AA-R (attB2) & $\begin{array}{l}\text { ggggaccactttgtacaagaaagctgggtTTAAATATTTGCCCAGGGCGCTGT } \\
\text { TCTTGCCTTATGCCTCGTTCCCTT }\end{array}$ & Vd_XacPNPc 9AA_n GFP \\
\hline GFP-VdAve1" 18AA-R & $\begin{array}{l}\text { TTATATCTGTCTAAATTCGATGTTGACCGCCTTATGCCTCGTTCCCT } \\
\text { TCCACTGATTTTTGTATAGTTCATCCATGC }\end{array}$ & SP_GFP_VdAve1 ${ }^{c} 18 \mathrm{AA}$ \\
\hline Avr9_VdAve1 $1^{\text {c18AA }}-\mathrm{R}$ & $\begin{array}{l}\text { TTATATCTGTCTAAATTCGATGTTGACCGCCTTATGCCTCGTTCCCT } \\
\text { TCCACTGATTATGTACACATTGGAGCTTA }\end{array}$ & SP_Avr9_VdAve1 ${ }^{\text {c } 18 \mathrm{AA}}$ \\
\hline
\end{tabular}


Recognition of Verticillium dahliae effector Ave1 by tomato Ve1

Table S2 (continued)

\begin{tabular}{|c|c|c|}
\hline Primer name & Oligonucleotide sequence $\left(5^{\prime} \rightarrow 3^{\prime}\right)$ & Description $^{\mathrm{a}}$ \\
\hline VdAve1 1 32AA-F & $\begin{array}{l}\text { ATGAAGCTTTCTACGCTTGGAGCCCTCATTTCATTGACTTCACTGG } \\
\text { TCACTGCCGCGACCATGATCTTATCT }\end{array}$ & SP_VdAve1 $1^{\text {c } 32 \mathrm{AA}}$ \\
\hline GFP-VdAve1 ${ }^{\text {c 32AA-F }}$ & GCATGGATGAACTATACAAAGCGACCATGATCTTATCT & SP_GFP_VdAve1c $32 \mathrm{AA}$ \\
\hline GFP-VdAve1 $1^{\text {c32AA-R }}$ & AGATAAGATCATGGTCGCTTTGTATAGTTCATCCATGC & SP_GFP_VdAve $1^{\text {c } 32 \mathrm{AA}}$ \\
\hline Avr9-VdAve1 ${ }^{c 32 A A}-F$ & TAAGCTCCAATGTGTACATGCGACCATGATCTTATCT & SP_Avr9_VdAve1 ${ }^{\text {c32AA }}$ \\
\hline Avr9-VdAve1 ${ }^{c 32 A A}-R$ & AGATAAGATCATGGTCGCATGTACACATTGGAGCTTA & SP_Avr9_VdAve1 ${ }^{c 32 A A}$ \\
\hline VdAve1 ${ }^{c 42 A A}-F$ & $\begin{array}{l}\text { ATGAAGCTTTCTACGCTTGGAGCCCTCATTTCATTGACTTCACTGG } \\
\text { TCACTGCCAAGACAACTGTTACCAAAGC }\end{array}$ & SP_VdAve $1^{\mathrm{c} 42 \mathrm{AA}}$ \\
\hline GFP-VdAve $1^{\text {c42AA-F }}$ & GCATGGATGAACTATACAAAAAGACAACTGTTACCAAAGC & SP_GFP_VdAve $1^{\text {c } 42 \mathrm{AA}}$ \\
\hline GFP-VdAve $1^{\text {c } 42 A A}-R$ & GCTTTGGTAACAGTTGTCTTTTTGTATAGTTCATCCATGC & SP_GFP_VdAve $1^{\text {c } 42 \mathrm{AA}}$ \\
\hline Avr9-VdAve1 ${ }^{c 42 A A-F}$ & TAAGCTCCAATGTGTACATAAGACAACTGTTACCAAAGC & SP_Avr9_VdAve1 ${ }^{\text {c42AA }}$ \\
\hline Avr9-VdAve1 ${ }^{c 42 A A}-R$ & GCTTTGGTAACAGTTGTCTTATGTACACATTGGAGCTTA & SP_Avr9_VdAve1 ${ }^{\text {c42AA }}$ \\
\hline GFP-VdAve $1^{\Delta 19-34-F}$ & GCATGGATGAACTATACAAATGCGGCGGCAGCAATCCC & SP_GFP_VdAve $1^{\Delta 19-34}$ \\
\hline GFP-VdAve $1^{\Delta 19-34-R}$ & GGGATTGCTGCCGCCGCATTTGTATAGTTCATCCATGC & SP_GFP_VdAve1 ${ }^{\Delta 19-34}$ \\
\hline Avr9-VdAve1 ${ }^{\Delta 19-34-F}$ & TAAGCTCCAATGTGTACATTGCGGCGGCAGCAATCCC & SP_Avr9_VdAve1 ${ }^{\Delta 19-34}$ \\
\hline Avr9-VdAve1 ${ }^{\Delta 19-34-R}$ & GGGATTGCTGCCGCCGCAATGTACACATTGGAGCTTA & SP_Avr9_VdAve1 ${ }^{\Delta 19-34}$ \\
\hline VdAve1 $1^{\text {n } 16 A A-R}$ (attB2) & ggggaccactttgtacaagaaagctgggtTTAGGCAGTGGGAAGGTA & SP_VdAve $1^{\text {n } 16 \mathrm{AA}}$ \\
\hline GFP-VdAve $1^{\text {n } 16 A A-R}$ & GGCAGTGGGAAGGTAGGGTGGGTTATAGTAGGATGCGGTCCCTAG & SP_GFP_VdAve $1^{\text {n } 16 \mathrm{AA}}$ \\
\hline Avr9-VdAve $1^{\text {n } 16 A A-R}$ & $\begin{array}{l}\text { GGCAGTGGGAAGGTAGGGTGGGTTATAGTAGGATGCGGTCCCTAGA } \\
\text { TCATGTACACATTGGAGCTTA }\end{array}$ & SP_Avr9_VdAve $1^{\text {n16AA }}$ \\
\hline VdAve1-F (PacI) & TTAATTAAAATGAAGCTTTCTACGCTTGGA & $\begin{array}{l}\text { For VdAve1, VdAve } 1^{\Delta 19-34} \\
\text { complementation strains }\end{array}$ \\
\hline VdAve1-R (NotI) & GCGGCCGCTCATATCTGTCTAAATTCGATGTTGA & $\begin{array}{l}\text { For VdAve1, VdAve } 1^{\Delta 19-34} \\
\text { complementation strains }\end{array}$ \\
\hline VdAve1 ${ }^{\Delta 126-134}-\mathrm{R}($ NotI) & GCGGCCGCTCACTTATGCCTCGTTCCCTTCCAC & $\begin{array}{l}\text { For VdAve } 1^{\Delta 126-134} \\
\text { complementation strains }\end{array}$ \\
\hline
\end{tabular}

aThe type of experiment for which the primers were used. 


\section{Chapter 3}

\section{Methods S1. Generation of binary expression vectors}

Constructs for the constitutive expression of VdAve1, SIPNP, VvPNP, FoAve1, CbAve1, ChAve1 and XacPNP have been described previously (de Jonge et al., 2012; Song et al., 2016). VnAve1 was amplified from $V$. nubilum cDNA by using the primers VnAve1-F (attB1) and VnAve1-R (attB2) listed in Table S2, and subsequently cloned into the entry vector pDONR 207 by using the Gateway ${ }^{\circledR}$ BP Clonase ${ }^{\circledR}$ II Enzyme Mix (Invitrogen, California, USA), and recombined into the Gateway-compatible destination vector pSol2092 (Zhang et al., 2013) to generate expression construct pSol2092::VnAve1.

Constructs encoding C-terminally tagged VdAve1 versions. To generate VdAve1 fused at the Cterminus to the green fluorescent protein (GFP), the VdAve1 coding sequence without stop codon was amplified by using primers VdAve1-F(attB1) and VdAve1( $\Delta$ SC)-R(attB2) listed in Table S2. The PCRamplified fragment was cloned into pDONR207 by using the Gateway ${ }^{\circledR}$ BP Clonase ${ }^{\circledR}$ II Enzyme Mix (Invitrogen, California, USA) to generate entry vector pDONR207::VdAve1 ( $\Delta S C$ ) verified by DNA sequencing (Eurofins Genomics, Ebersberg, Germany). Subsequently, pDONR207::VdAve1 $(\Delta S C)$ was transferred into the Gateway-compatible destination vector pSol2095 (C-terminal GFP tag) (Zhang et al., 2013) by using Gateway ${ }^{\circledR}$ LR Clonase ${ }^{\circledR}$ II Enzyme Mix (Invitrogen, California, USA) to generate Cterminally GFP-tagged VdAve1 (VdAve1_c GFP; Figure 2A). Similarly, pDONR207::VdAve1 $(\Delta S C)$ was recombined into the Gateway-compatible destination vectors pGWB14, pGWB8, pGWB17, pGWB20 and pGBW11 (Nakagawa et al., 2007) to generate C-terminally affinity-tagged VdAve1 versions VdAve1_c 3xHA, VdAve1_c 6xHIS, VdAve1_4xMyc, VdAve1_c 10xMyc, VdAve1_c FLAG (Figure 2A), respectively.

Constructs encoding N-terminally fused VdAve1 versions. To fuse different tags (GFP, HA, Myc and mature Avr9) to the N-terminus of mature VdAve1, the corresponding tag coding sequence lacking the stop codon was fused at the N-terminus to the sequence encoding the signal peptide of VdAve1 to ensure extracellular targeting, and at the C-terminus to the mature VdAve1 protein. DNA fragments for constructs VdAve1_n GFP, VdAve1_n HA, VdAve1_n Myc, VdAve1_n Avr9, SP_GFP and SP_Avr9 ( Figure 2C) were obtained by gene synthesis (Eurofins MWG Operon, Ebersberg, Germany), and cloned into the entry vector pDONR 207 and subsequently transferred into the Gateway-compatible destination vector pSol2092 (Zhang et al., 2013).

Constructs encoding VdAve1 C-terminal deletions. To generate VdAve1 truncations VdAve1 ${ }^{\Delta 93-}$ 134, VdAve1 $1^{\Delta 103-134}$, VdAve1 $1^{\Delta 17-134}$, VdAve1 $1^{\Delta 126-134}$ and VdAve1 ${ }^{\mathrm{n} 16 \mathrm{AA}}$, these DNA fragments were amplified from plasmid pSol2092::VdAve1 (Zhang et al., 2013) by using the forward primer VdAve1-F (attB1) in combination with the reverse primers VdAve1 ${ }^{\Delta 93-134-R}$ (attB2), VdAve1 ${ }^{\Delta 103-134-R}$ (attB2), VdAve1 ${ }^{\Delta 117-134-R}$ (attB2), VdAve1 $1^{\Delta 126-134}-\mathrm{R}$ (attB2) and VdAve1 ${ }^{\mathrm{n}}$ 16AA-R (attB2) (Table S2), respectively. A fragment SP_GFP_VdAve1 ${ }^{c 9 A A}$ was amplified by PCR from plasmid SP_GFP using the primers VdAve1-F (attB1) and 
Recognition of Verticillium dahliae effector Ave1 by tomato Ve1

GFP-VdAve1c 9AA-R (attB2) (Table S2). Vd_XacPNPc 9AA, Xac_VdAve1 ${ }^{c 9 A A}, S P_{-} V d A v e 1^{c 9 A A}, S P_{-} V d A v e 1^{c 18 A A}$, and VdAve $1^{\Delta 19-34}$ were obtained by gene synthesis (Eurofins MWG Operon, Ebersberg, Germany), and cloned into the entry vector pDONR 207 and subsequently transferred into the vector pSol2092 (Zhang et al., 2013). The AVNIEFRQI peptides were synthesized by GenScript corporation (GenScript, New Jersey, USA).

Constructs encoding N-terminally GFP-tagged VdAve1 homologs and chimeras. To generate VnAve1, SIPNP, VvPNP, FoAve1, CbAve1, ChAve1, XacPNP and Xac_VdAve1c9AA fused GFP tag to the Nterminus, the GFP coding sequence lacking the stop codon was fused at the N-terminus to the sequence encoding the signal peptide of VdAve1, and at the C-terminus to the mature VdAve1 homologs. The SP_GFP fragment and DNA fragments for the mature VdAve1 homologs were separately amplified and fused by the overlapping PCR. Seven SP_GFP fragments were amplified from plasmid SP_GFP by using the forward primer VdAve1-F (attB1) in combination with the reverse primers GFP-VnAve1-R, GFPSIPNP-R, GFP-VvPNP-R, GFP-FoAve1-R, GFP-CbAve1-R, GFP-ChAve1-R and GFP-XacPNP-R (Table S2). Eight fragments VnAve1, SIPNP, VvPNP, FoAve1, CbAve1, ChAve1, XacPNP and Xac_VdAve1c9AA were amplified from plasmids VnAve1, SIPNP, VvPNP, FoAve1, CbAve1, ChAve1, XacPNP and Xac_VdAve1c9AA by using the forward primers GFP-VnAve1-R, GFP-SIPNP-R, GFP-VvPNP-R, GFP-FoAve1-R, GFP-CbAve1R, GFP-ChAve1-R, GFP-XacPNP-R, GFP-XacPNP-R in combination with the corresponding reverse primers VnAve1-R (attB2), SIPNP-R (attB2), VvPNP-R(attB2), FoAve1-R (attB2), CbAve1-R (attB2), ChAve1-R (attB2) and XacPNP-R (attB2), VdAve1-R (attB2) (Table S2). Subsequently, eight desired DNA fragments were obtained by overlapping PCR by using the forward primer VdAve1-F (attB1) in combination with the corresponding reverse primers VnAve1-R (attB2), SIPNP-R (attB2), VvPNP-R (attB2), FoAve1-R (attB2), CbAve1-R (attB2), ChAve1-R (attB2), XacPNP-R (attB2), and XacPNP-R (attB2) (Table S2), and cloned into the entry vector pDONR 207 and subsequently recombined into the vector pSol2092 (Zhang et al., 2013). To generate VdAve1 ${ }^{\Delta 126-134}$, Vd_XacPNPc 9AA fused GFP tag to the Nterminus, the GFP coding sequence lacking the stop codon was fused at the N-terminus to the sequence encoding the signal peptide of VdAve1, and at the C-terminus to the VdAve1 chimeras. Two desired DNA fragments were amplified from plasmid VdAve1_n GFP by using the forward primer VdAve1-F (attB1) in combination with the reverse primers VdAve1 ${ }^{\Delta 126-134}-\mathrm{R}$ (attB2) and GFP-Vd_XacPNPc 9AA-R (attB2) (Table S2), and cloned into the vector pDONR 207 and subsequently transferred into the vector pSol2092 (Zhang et al., 2013).

Constructs encoding C- or N-terminus. To generate constructs SP_VdAve1c 32AA, SP_VdAve1 ${ }^{\text {c } 42 \mathrm{AA}}$, DNA fragments were amplified by the $1^{\text {st }}$ round PCR using plasmid VdAve1 as templates and the reverse

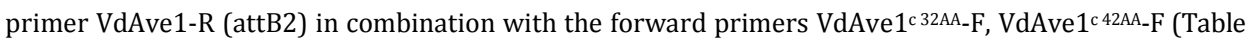




\section{Chapter 3}

S2), and followed by the $2^{\text {nd }}$ round PCR using primers VdAve1-F (attB1) and VdAve1-R (attB2) (Table

S2). The resulting PCR fragments were cloned into the entry vector pDONR 207, sequenced and subsequently recombined into the vector pSol2092 (Zhang et al., 2013).

Constructs encoding N-terminally GFP or Avr9-fused C- or N-terminus. To generate SP_GFP_VdAve1 ${ }^{1} 18 \mathrm{AA}, \mathrm{SP}_{-}$GFP_VdAve1 ${ }^{\mathrm{n} 16 \mathrm{AA}}$, DNA fragments were amplified by the $1^{\text {st }}$ round PCR using plasmid SP_GFP as templates and the forward primer VdAve1-F (attB1) in combination with the forward primers GFP-VdAve1 $1^{\text {c } 18 A \mathrm{~A}-\mathrm{R}}$ and GFP-VdAve $1^{\mathrm{n} 16 \mathrm{AA}-\mathrm{R}}$ (Table S2), respectively, and followed by the $2^{\text {nd }}$ round PCR using primers VdAve1-F (attB1) and VdAve1-R (attB2) (Table S2). To generate constructs

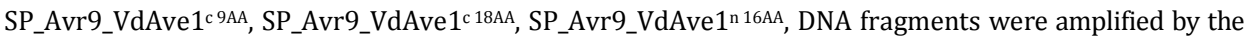
$1^{\text {st }}$ round PCR using plasmid SP_Avr9 as template and the forward primer VdAve1-F (attB1) in combination with the forward primers Avr9-VdAve1 ${ }^{\text {c }}$ AA-R, Avr9-VdAve1 ${ }^{c}$ 18AA-R and Avr9-VdAve1 ${ }^{\text {n 16AA- }}$ $\mathrm{R}$ (Table S2), respectively, and followed by the $2^{\text {nd }}$ round PCR using primers VdAve1-F (attB1) and VdAve1-R (attB2) (Table S2). The resulting PCR fragments were cloned into the vector pDONR 207, sequenced and subsequently transferred into the vector pSol2092 (Zhang et al., 2013). To construct SP_GFP_VdAve1 ${ }^{1}$ 32AA, SP_GFP_VdAve1 ${ }^{c}$ 42AA, SP_GFP_VdAve1 ${ }^{\Delta 19-34}, S_{-}$_Avr9_VdAve1 ${ }^{\text {c } 32 A A}$, SP_Avr9_VdAve1 ${ }^{c}$ ${ }^{42 A A}$ and SP_Avr9_VdAve1 $1^{\Delta 19-34}$, DNA fragments SP_VdAve1 ${ }^{c 32 A A}, S P_{-} V d A v e 1^{c 42 A A}, V d A v e 1^{419-34}, S P_{-} G F P$ and $S P_{-} A v r 9$ were separately amplified and fused by the overlapping PCR. Fragments $S P_{-} G F P$ and $S P_{-} A v r 9$ were amplified from plasmids SP_GFP and SP_Avr9 by using the forward primer VdAve1-F (attB1) in combination with the reverse primers GFP-VdAve1 ${ }^{c}$ 32AA-R, GFP-VdAve1c ${ }^{42 A A}-R$, GFP-VdAve1 ${ }^{\Delta 19-34-R,}$

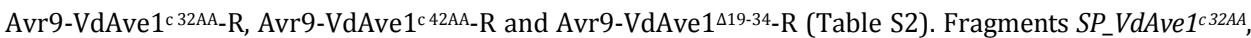
SP_VdAve1 ${ }^{c 42 A A}$ and VdAve $1^{\Delta 19-34}$ were amplified from plasmid VdAve1 using the reverse primer VdAve1-

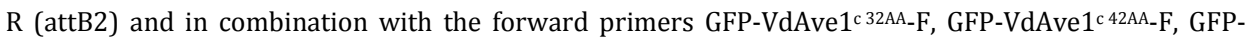
VdAve1 199-34-F, Avr9-VdAve1 ${ }^{c}$ 32AA-F, Avr9-VdAve1c ${ }^{42 A A-F}$ and Avr9-VdAve1 ${ }^{\Delta 19-34-F}$ (Table S2). Subsequently, six desired DNA fragments $S P_{-} G F P_{-} V d A v e 1^{c 32 A A}, S P_{-} G F P_{-} V d A v e 1^{c 42 A A}, S P_{-} G F P_{-} V d A v e 1^{419-34}$, SP_Avr9_VdAve1 ${ }^{c 32 A A}, P_{-}$Avr9_VdAve1 ${ }^{42 A A}$ and SP_Avr9_VdAve1 ${ }^{419-34}$ were obtained by overlapping PCR using the primers VdAve1-F (attB1) and VdAve1-R (attB2) (Table S2), and cloned into the entry vector pDONR 207 and subsequently recombined into the vector pSol2092 (Zhang et al., 2013).

All the destination vectors were transformed into Agrobacterium tumefaciens strain GV3101 (pMP90) by electroporation. 


\section{REFERENCES}

de Jonge R, van Esse HP, Maruthachalam K, Bolton MD, Santhanam P, Saber MK, Zhang Z, Usami T, Lievens B, Subbarao KV, Thomma BPHJ (2012) Tomato immune receptor Ve1 recognizes effector of multiple fungal pathogens uncovered by genome and RNA sequencing. Proc Natl Acad Sci USA 109: 5110-5115.

Nakagawa T, Kurose T, Hino T, Tanaka K, Kawamukai M, Niwa Y, Toyooka K, Matsuoka K, Jinbo T, Kimura T (2007) Development of series of gateway binary vectors, pGWBs, for realizing efficient construction of fusion genes for plant transformation. J Biosci Bioeng 104: 34-41.

Song Y, Zhang Z, Seidl MF, Majer A, Jakse J, Javornik B, Thomma BPHJ (2016) Broad taxonomic characterization of Verticillium wilt resistance genes reveals an ancient origin of the tomato Ve1 immune receptor. Mol Plant Pathol 18: 195-209.

Zhang Z, Fradin E, de Jonge R, van Esse HP, Smit P, Liu C-M, Thomma BPHJ (2013) Optimized agroinfiltration and virus-induced gene silencing to study Ve1-mediated Verticillium resistance in tobacco. Mol Plant-Microbe Interact 26: 182-190. 



\section{Chapter 4}

\section{Broad taxonomic characterization of Verticillium wilt resistance genes reveals ancient origin of the tomato Ve1 immune receptor}

Yin Song1, Zhao Zhang1, Michael F. Seidl ${ }^{1}$, Aljaz Majer ${ }^{2}$, Jernej Jakse², Branka Jovornik $^{2}$ and Bart P.H.J. Thomma ${ }^{1}$

${ }^{1}$ Laboratory of Phytopathology, Wageningen University, Droevendaalsesteeg 1, 6708 PB Wageningen, the Netherlands

2Department of Agronomy, Biotechnical Faculty, University of Ljubljana, Jamnikarjeva 101, SI-1000 Ljubljana, Slovenia

This chapter has been published as:

Song Y, Zhang Z, Seidl MF, Majer A, Jakse J, Javornik B, Thomma BPHJ (2017) Broad taxonomic characterization of Verticillium wilt resistance genes reveals ancient origin of the tomato Ve1 immune receptor. Mol Plant Pathol 18: 195-209. 


\section{Chapter 4}

\section{ABSTRACT}

Plant pathogenic microbes secrete effector molecules to establish themselves on their hosts, whereas plants use immune receptors to try and intercept such effectors in order to prevent pathogen colonization. The tomato cell surfacelocalized receptor Ve1 confers race-specific resistance against race 1 strains of the soil-borne vascular wilt fungus Verticillium dahliae that secrete the Ave1 effector. Here, we describe the cloning and characterization of Ve1 homologues from tobacco (Nicotiana glutinosa), potato (Solanum tuberosum), wild eggplant (Solanum torvum) and hop (Humulus lupulus), and demonstrate that particular Ve1 homologues govern resistance against $V$. dahliae race 1 strains through recognition of the Ave1 effector. Phylogenetic analysis shows that Ve1 homologs are widely distributed in land plants. Thus, our study suggests an ancient origin of the Ve1 immune receptor in the plant kingdom. 


\section{INTRODUCTION}

In order to activate immune responses that ward off invading microorganisms, plants employ immune receptors that detect pathogen(-induced) ligands of various nature (Boller and Felix, 2009; Thomma et al., 2011). The recognition of such ligands by immune receptors results in the activation of defense responses, which are often accompanied by a hypersensitive response (HR) in which necrosis of plant tissue surrounding the site of attempted penetration is sacrificed to restrict further pathogen invasion.

Verticillium wilts are vascular wilt diseases that are caused by soil-borne fungal pathogens that belong to the Verticillium genus. Verticillium dahliae is the most notorious species that can infect hundreds of dicotyledonous hosts (Fradin and Thomma, 2006; Inderbitzin et al., 2011). In tomato (Solanum lycopersicum), the Ve locus that confers race-specific resistance against Verticillium has been characterized (Fradin et al., 2009; Kawchuk et al., 2001). This locus contains two closely linked and inversely oriented genes, $V e 1$ and $V e 2$, which encode extracellular leucine-rich repeat receptor-like proteins (eLRR-RLPs) (Kawchuk et al., 2001; Wang et al., 2008, 2010). Of these, only Ve1 was found to provide $V$. dahliae resistance in tomato (Fradin et al., 2009). Interestingly, interfamily transfer of Ve1 from tomato to Arabidopsis resulted in Verticillium resistance in the latter species (Fradin et al., 2011, 2014; Zhang et al., 2014), implying that the underlying immune signaling pathway is conserved (Fradin et al., 2011; Thomma et al., 2011).

Comparative genomics of $V$. dahliae race 1 and race 2 strains identified the Ave1 effector that activates Ve1-mediated immunity (de Jonge et al., 2012). Interestingly, Ave1 homologs were found in the bacterial plant pathogen Xanthomonas axonopodis pv. citri (XacPNP) and in the plant pathogenic fungi Colletotrichum higginsianum (ChAve1), Cercospora beticola (CbAve1), and Fusarium oxysporum f. sp. lycopersici (FoAve1), and these homologs are differentially recognized by Ve1 (de Jonge et al., 2012). During optimization of an agroinfiltration assay in tobacco for functional analysis of Ve1 signaling, we found that expression of Ave1 in leaves of Nicotiana glutinosa triggered an HR, suggesting that this species contains an endogenous Ve1 allele (Zhang et al., 2013a). Indeed, inoculation experiments revealed that $N$. glutinosa is resistant to race $1 \mathrm{~V}$. dahliae, while an 


\section{Chapter 4}

Ave1 deletion strain was able to cause Verticillium wilt disease on these plants (Zhang et al., 2013a).

So far, several Ve1 homologs were identified within and outside the Solanaceae family, such as SIVe1 from Solanum lycopersicoides (Chai et al., 2003), StVe1 from S. tuberosum (Simko et al., 2004a), StVe and StoVe1 from S. torvum (Fei et al., 2004; Liu et al., 2012), mVe1 from Mentha longifolia (Vining and Davis, 2009), Vr1 from Lactuca sativa (Hayes et al., 2011), VvVe from Vitis vinifera (Tang et al., 2016) and GbVe, Gbve1, Gbvdr5 and Gbvdr3 from Gossypium barbadense (Chen et al., 2016; Yang et al., 2014; Zhang et al., 2011; Zhang et al., 2012). However, functionality of these homologs against Verticillium wilt often remains obscure. Here, we describe the cloning and functional characterization of Verticillium wilt resistance genes from tobacco (N. glutinosa), potato (S. tuberosum), wild eggplant (S. torvum) and hop (Humulus lupulus), and demonstrate that particular Ve1 homologs govern resistance against $V$. dahliae race 1 strain through the recognition of the Ave1 effector.

\section{RESULTS}

\section{Isolation of $\mathrm{NgVe1}$ from Nicotiana glutinosa}

In our first attempt to clone the previously identified Ve1 homolog from N. glutinosa (Zhang et al., 2013a), a single cDNA fragment of 2800 bp was obtained using primers that were designed on the tomato Ve1 sequence (Table S1). To obtain the full-length $\mathrm{N}$. glutinosa $\mathrm{Ve1}$ ( $\mathrm{NgVe1}$ ) transcript sequence, 3' rapid amplification of cDNA end (RACE) polymerase chain reaction (PCR) was performed, resulting in a single fragment of approximately $1200 \mathrm{bp}$. Likewise, a fragment of approximately 640 bp was amplified with 5' RACE (Methods S1). The sequences of the three fragments were aligned to deduce the full-length $\mathrm{NgVe1}$ cDNA sequence. Subsequently, a pair of $N g V e 1$-specific primers (NgVe1-F and NgVe1-R; Table S1) was designed and amplicons amplified from $N$. glutinosa cDNA and genomic DNA were sequenced, indicating that both amplicons are identical (GenBank accession: KT895339) and that $\mathrm{NgVe1}$ is an intronless gene.

The full-length cDNA of $\mathrm{NgVe} 1$ is $3225 \mathrm{bp}$, and contains a predicted translation initiation site (ATG) at nucleotide position 34 and a stop codon (TGA) at nucleotide position 3178, resulting in a single open reading frame of $3147 \mathrm{bp}$. The predicted 
NgVe1 protein comprises 1048 amino acids (GenBank accession: ALK26499), and shares an overall identity of $76 \%$ with tomato Ve1 (Figure S1). Immunoblotting analysis using GFP antibody displayed clear signals for NgVe1-GFP and Ve1-GFP in transiently transformed tobacco leaves (Figure S2).

\section{Co-expression of Ave1 and $\mathrm{NgVe1}$ induces an HR in $\mathrm{N}$. tabacum}

Recently, an optimized agroinfiltration assay has been developed for Ve1-mediated immune signaling in $N$. tabacum, revealing a swift HR upon co-expression of tomato Ve1 with $V$. dahliae Ave1 (Zhang et al., 2013a). To test functionality of $\mathrm{NgVe1}$, coexpression with Ave1 upon agroinfiltration in N. tabacum was performed. At 5 days post infiltration, the infiltrated leaves developed clear necrosis, and the HR induced upon co-expression of $\mathrm{NgVe1}$ and Ave1 was as strong as HR induced upon coinfiltration of tomato $V e 1$ and Ave1, for which the complete infiltrated areas became fully necrotic (Figure 1A). In contrast, agroinfiltration of $\mathrm{NgVe1}$ or Ave1 alone did not induce necrosis (Figure 1A). These data strongly suggest that $\mathrm{NgVe1}$ is a functional homolog of tomato $\mathrm{Ve} 1$.

\section{Targeting of $\mathrm{NgVe1}$ expression in $\mathrm{N}$. glutinosa compromises Ave1-induced HR, but not Verticillium resistance}

To investigate the role of $\mathrm{NgVe1}$ in $\mathrm{N}$. glutinosa Verticillium resistance, we used virus-induced gene silencing (VIGS). Tobacco rattle virus (TRV)-based VIGS is a well-established method for gene functional analysis in several Solanaceae species, also for investigation of Ve1-mediated Verticillium resistance (Fradin et al., 2009; Senthil-Kumar et al., 2007; Zhang et al., 2013a). In an attempt to establish VIGS in $N$. glutinosa, a 1:1 mixture of Agrobacterium tumefaciens cultures carrying $p T R V 1$ and pTRV2::PDS to target the phytoene desaturase (PDS) gene was infiltrated into cotyledons of $N$. glutinosa plants. Visible photobleaching symptoms were observed in all agroinfiltrated $N$. glutinosa plants by 4 weeks post infiltration (Figure S3A), albeit that a strongly varying degree of photobleaching was observed (Figure S3A). Nevertheless, a recombinant TRV vector was designed to target $\mathrm{NgVe1}$ expression (pTRV2::NgVe1). As a negative control, a construct (pTRV2::GUS) containing a fragment of the $\beta$-glucuronidase (GUS) gene was used. At 4 weeks after TRV infection, mature leaves were agroinfiltrated to express Ave1, with VdNLP1 as a 


\section{Chapter 4}

positive control (Santhanam et al., 2013) and the functionally and structurally unrelated effector Avr9 from the tomato leaf mould pathogen Cladosporium fulvum as a negative control (van Kan et al., 1991; Van der Hoorn et al., 2000). Agroinfiltration of Ave1 in N. glutinosa upon GUS targeting resulted in a clear HR within 5 days, confirming that TRV infection did not compromise Ve1-mediated HR (Figure 1B). However, targeting of $\mathrm{NgVe1}$ expression in $\mathrm{N}$. glutinosa significantly compromised HR upon expression of Ave1 (Figure 1B). As expected, VdNLP1mediated cell death was not compromised upon targeting of $\mathrm{NgVe1}$ expression whereas Avr9 expression never triggered HR (Figure 1B).

A

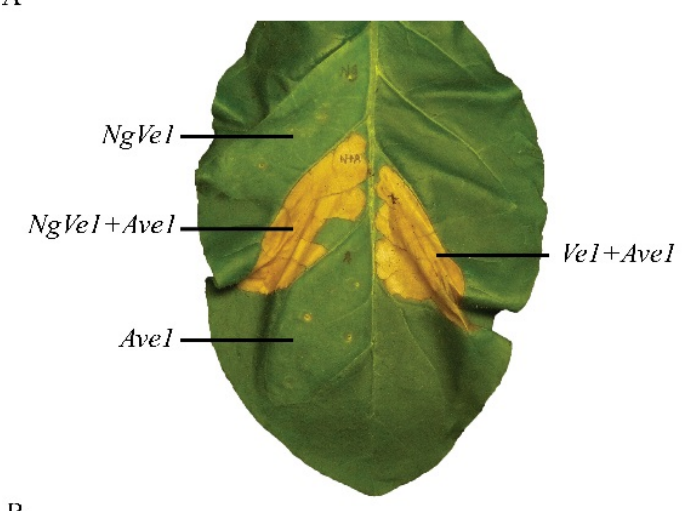

B

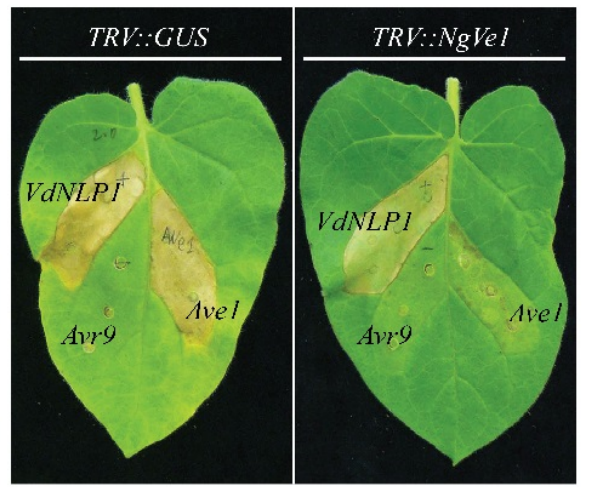

Figure 1. Expression of $\mathrm{NgVe1}$ in tobacco mediates the Ave1-triggered hypersensitive response (HR). (A) Co-expression of $\mathrm{NgVe} 1$ and Ave1 in tobacco results in an HR. Pictures were taken at 5 days post infiltration, and show representative leaves of at least three independent assays. As a positive control, HR was induced upon co-infiltration of Ve1 and Ave1. As a negative control, NgVe1 and Ave1 were expressed separately. (B) Ave1-triggered HR, but not the VdNLP1-mediated cell death is attenuated in NgVe1-silenced Nicotiana glutinosa plants, while Avr9 does not trigger cell death. 
To test the role of $\mathrm{NgVe1}$ in Verticillium resistance, 3 weeks after TRV inoculated plants were challenged either with $V$. dahliae race 1 strain JR2 (Faino et al., 2015), or a transformant from which the Ave1 gene had been deleted ( $V$. dahliae JR2 $\Delta$ Ave1; de Jonge et al., 2012), and monitored for disease development (stunting, wilting, chlorosis and necrosis) up to 14 days post inoculation (dpi). As expected, no disease symptoms were observed in N. glutinosa plants upon GUS targeting and subsequent mock-inoculation or upon inoculation with the V. dahliae JR2, whereas the Ave1 deletion strain caused clear Verticillium wilt disease (Figure. S3B). However, unexpectedly, in repeated assays also no Verticillium wilt symptoms were observed upon $\mathrm{NgVe1}$ targeting and subsequent inoculation with $\mathrm{V}$. dahliae (Figure S3B). However, in line with the extremely variable photobleaching (Figure $\mathrm{S} 3 \mathrm{~A})$, assessment of the silencing efficiency revealed only a slight reduction in $N g V e 1$ expression in $\mathrm{NgVe1}$-targeted $\mathrm{N}$. glutinosa plants when compared with GUSsilenced plants (Figure S3C), and attempts to increase the silencing efficiency were unsuccessful. These results confirm previous observations that $N$. glutinosa is not very amenable to TRV-based VIGS (Senthil-Kumar et al., 2007; Zhang et al., 2013a), and, furthermore, suggest that the moderate silencing efficiency that we obtained in our experiments is sufficiently high to compromise the NgVe1-mediated HR, but insufficient to compromise $\mathrm{NgVe1}$-mediated resistance.

\section{Expression of $\mathbf{N g V e 1}$ in Arabidopsis confers Verticillium resistance}

As TRV-based VIGS appeared not to be very suitable for gene functional analysis in $N$. glutinosa, we pursued other strategies to functionally characterize $\mathrm{NgVe1}$. We have previously shown that interfamily transfer of tomato Ve1 to Arabidopsis resulted in resistance against race 1 Verticillium strains, providing a relatively fast method to assess Ve1 functionality (Fradin et al., 2011; 2014; Zhang et al., 2014). To further confirm functionality of $\mathrm{NgVe1}$ in Verticillium resistance, heterologous expression of $\mathrm{NgVe1}$ in Arabidopsis was obtained (Figure S4). No obvious developmental alterations were observed in transgenic plants when compared with wild-type plants (Figure 2A) and, subsequently three independent NgVe1transgenic lines (NgVe1-1, NgVe1-2 and NgVe1-3) as well as transgenic plants expressing tomato Ve1 (Fradin et al., 2011) and non-transgenic controls were inoculated with $V$. dahliae JR2. Interestingly, like tomato Ve1-expressing plants, 


\section{Chapter 4}

A

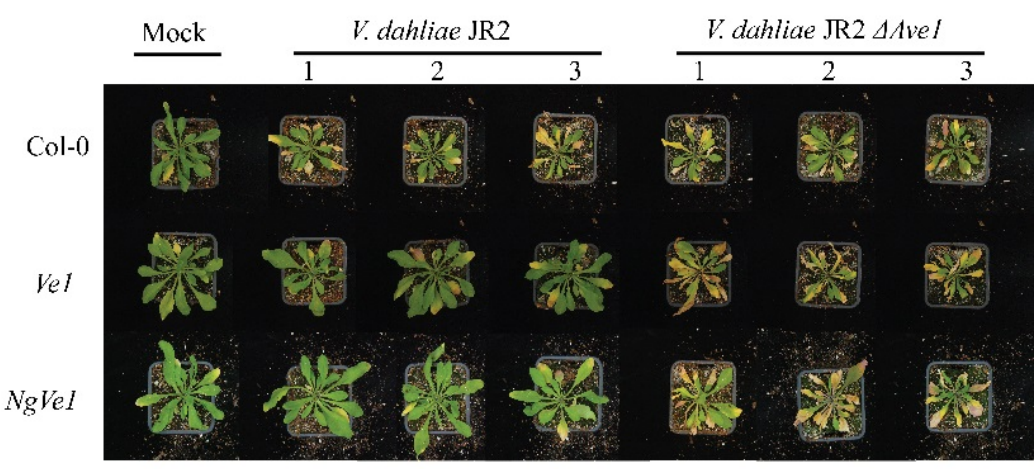

B

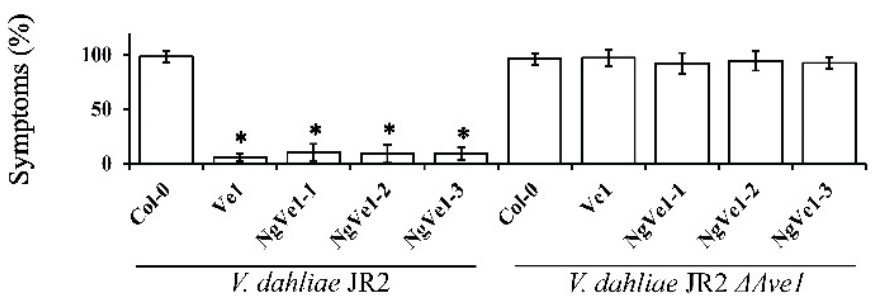

$\mathrm{C}$

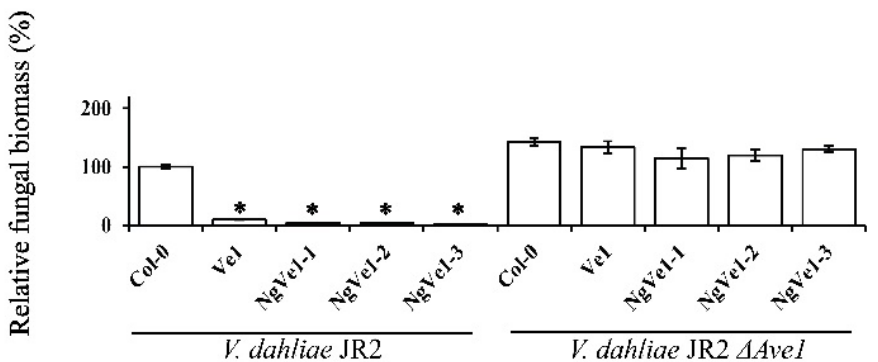

Figure 2. Expression of $\mathrm{NgVe1}$ in Arabidopsis mediates resistance against race 1 Verticillium dahliae. (A) Typical appearance of non-transgenic Arabidopsis and transgenic lines that constitutively express $\mathrm{NgVe} 1$ upon mock-inoculation or inoculation with V. dahliae strain JR2 or V. dahliae JR2 $\Delta$ Ave1 at 21 days post inoculation (dpi). (B) Quantification of Verticillium wilt symptoms in Arabidopsis Col-0 and transgenic plants at $21 \mathrm{dpi}$. Bars represent quantification symptom development as percentage of diseased rosette leaves with standard deviation, with Col-0 (control) set to $100 \%$. (C) Fungal biomass determined by quantitative PCR (qPCR) in Arabidopsis Col-0 and transgenic plants at $21 \mathrm{dpi}$. Bars represent Verticillium internal transcribed spacer (ITS) transcript levels relative to AtRuBisCo (RuBisCo, ribulose-1,5-bisphosphate carboxylase/oxygenase) transcript levels (for equilibration) with standard deviation in a sample of five pooled plants. The fungal biomass in Col-0 (control) is set to $100 \%$. Three independent lines are shown (1,2 and 3). Asterisks indicate significant differences when compared with Col-0 $(P<0.05)$. Ve1 transgenic plants were used as a positive control. The data shown are representative of at least three independent experiments. 
$\mathrm{NgVe} 1$-transgenic plants were clearly resistant to race $1 \mathrm{~V}$. dahliae as significantly less Verticillium wilt symptoms were observed when compared with nontransgenic control plants (Figure 2A, B). In contrast, $\mathrm{NgVe1}$ and $\mathrm{Ve} 1$ transgenic plants were as diseased as non-transgenic controls upon inoculation with the Ave1 deletion strain (Figure 2A, B). These data are further supported by fungal biomass quantifications that revealed significantly reduced fungal accumulation in $\mathrm{NgVe1}$ transgenic and Ve1-expressing Arabidopsis plants for $V$. dahliae carrying Ave1, but not for the Ave1 deletion mutant, when compared with wild-type Arabidopsis plants (Figure 2C). Collectively, these data confirm that NgVe1 acts as a functional homologue of tomato $\mathrm{Ve} 1$ that recognizes race $1 \mathrm{~V}$. dahliae.

\section{Cloning and functional analysis of $\mathrm{Ve} 1$ homologues from potato and wild eggplant}

$V e$ gene homologues occur in the solanaceous species S. lycopersicoides (SIVe1; Chai et al., 2013) and the wild eggplant species S. torvum (StVe and StoVe1; Fei et al., 2004; Liu et al., 2012). Moreover, in tetraploid potato (S. tuberosum), a quantitative trait locus (QTL) for Verticillium resistance was identified using the tomato Ve1 gene as a probe. This locus was found to contain at least 11 genes, all putatively encoding LRR-type receptor-like proteins (Simko et al., 2004a). The tomato and potato genomes are highly collinear and the QTL locus was mapped to a region on potato chromosome 9 that is syntenic to the short arm of the tomato chromosome 9 that carries Ve1 and Ve2 (Diwan et al., 1999; Simko et al., 2004b). Subsequently, this Verticillium resistance QTL locus was annotated and found to contain two predicted receptor-like protein 12-like genes [National Center for Biotechnology Information (NCBI): XM_006362308 and XM_006362309] in the genome sequence of $S$. tuberosum group Phureja DM 1-3 516 R44 (Xu et al., 2011). Here, the coding sequences (CDSs) of $V e$ gene homologues were amplified from cDNA of the heterozygous diploid potato breeding line $S$. tuberosum group Tuberosum RH 89039-16 (Xu et al., 2011), sequenced and submitted to NCBI as StuVe1 and StuVe2 (GenBank accessions: KT946795 and KT946797) (Methods S1). The predicted StuVe1 and StuVe2 proteins are composed of 1053 and 1138 amino acids (GenBank accessions: ALK26501 and ALK26503), respectively, and share 87\% and 84\% 


\section{Chapter 4}

identity with tomato Ve1 and $81 \%$ and $91 \%$ identity with tomato Ve2, respectively, and $82 \%$ identity with each other (Figure S1).

A
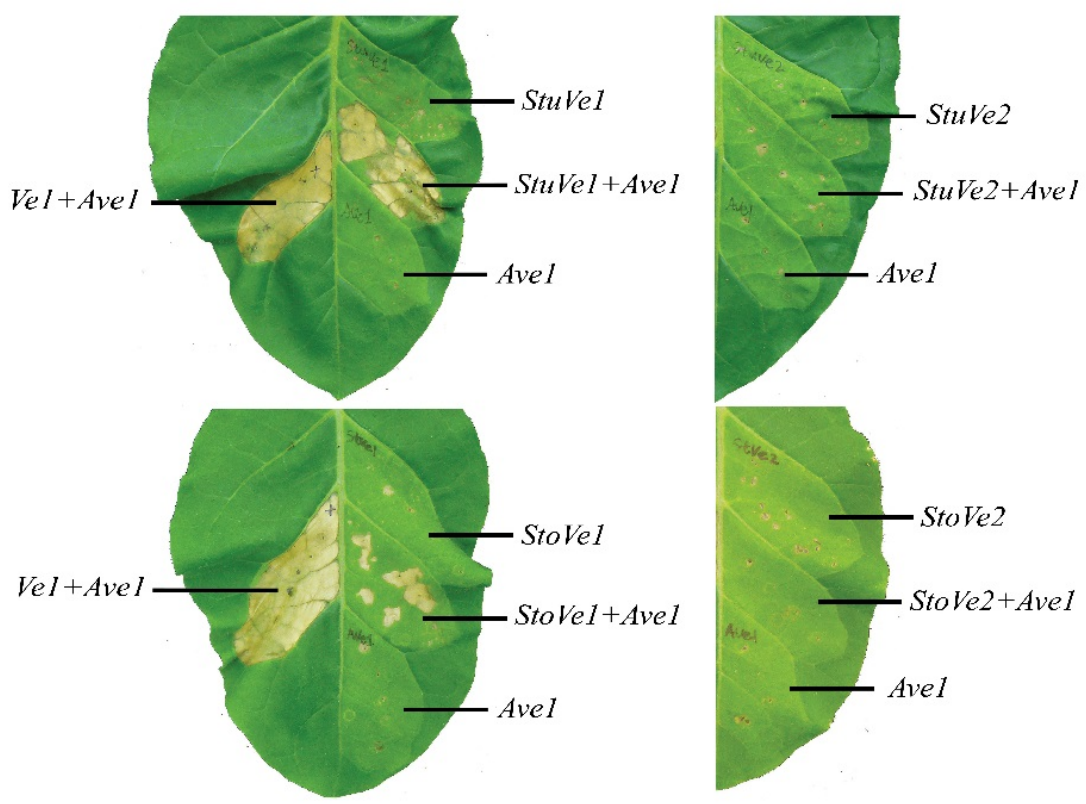

B

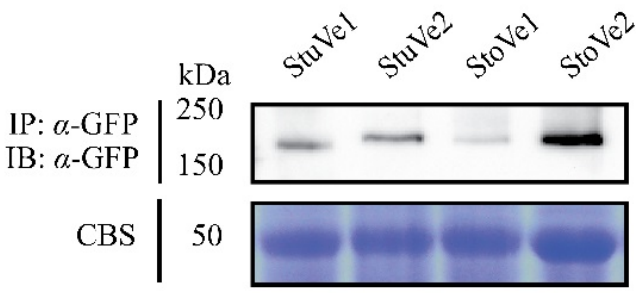

Figure 3. StuVe1 and StoVe1, but not StuVe2 and StoVe2, recognize Ave1 in Nicotiana tabacum. (A) Co-expression of StuVe1 and StoVe1, but not StuVe2 and StoVe2, with Ave1 in tobacco induces signs of a relatively weak hypersensitive response (HR). Pictures were taken at 5 days post infiltration, and show representative leaves of at least three independent assays. As a positive control, HR was induced upon co-infiltration of Ve1 and Ave1. As a negative control, StuVe1, StuVe2, StoVe1, StoVe2, and Ave1 were expressed separately. (B) Green fluorescent protein (GFP)-tagged StuVe1, StuVe2, StoVe1, and StoVe2 proteins are present in planta by immunopurification (IP) using GFP-affinity beads, followed by immunoblotting (IB) using $\alpha$-GFP antibody. Coomassie Blue Staining (CBS) of the blotting containing total protein extracts showed equal loading in each lane based on the 50-kDa RuBisCo (ribulose-1,5bisphosphate carboxylase/oxygenase) band. 
To study the composition of the Ve locus in wild eggplant, the CDSs of Ve gene homologs were cloned from the cDNA of the Verticillium-resistant S. torvum genotype Tuolubamu, sequenced and deposited at NCBI as StoVe1 and StoVe2 (GenBank accessions: KT946794 and KT946796) (Methods S1). The predicted StoVe1 and StoVe2 proteins comprise 1051 and 1135 amino acids (GenBank accessions: ALK26500 and ALK26502), respectively, and share 83\% and 80\% identity with tomato Ve1 and $81 \%$ and $85 \%$ identity with tomato Ve2, respectively, and $92 \%$ identity with each other (Figure S1).

To check functionality of StuVe1, StuVe2, StoVe1 and StoVe2, mature tobacco leaves were co-infiltrated with a 1:1 mixture of $A$. tumefaciens cultures carrying Ave 1 and the various $V e 1$ homologues. Intriguingly, agroinfiltration in at least three independent assays revealed that expression of Ave1 together with StuVe1 or StoVe1 induced signs of a weak HR at 5 days post infiltration (Figure 3A). However, when compared with the HR induced upon co-agroinfiltration of tomato Ve1 and Ave1, only a minor part of the infiltrated region developed necrosis (Figure 3A). Nevertheless, agroinfiltration of Ave1 with StuVe2 or StoVe2 induced no such responses at all (Figure 3A). Immunoblotting confirmed that the StuVe1, StuVe2, StoVe1, and StoVe2 fusion proteins were expressed (Figure 3B).

As VIGS-based gene silencing in potato genotype Tuberosum RH 89-039-16 and wild eggplant genotype Tuolubamu has not been established, we did not attempt VIGS-based assays to test the role of these Ve1 homologuess in Verticillium resistance. Rather, heterologous expression in Arabidopsis was pursued (Figure S4). No developmental alterations were observed in transgenic plants when compared with Ve1-expressing and wild-type plants (Figure 4A) and three independent transgenic lines expressing StuVe1, StuVe2, StoVe1 or StoVe2 were assayed for V. dahliae resistance. Intriguingly, despite the weak HR observed upon agroinfiltration together with Ave1 in N. tabacum, StoVe1- and StuVe1-expressing plants were clearly resistant to race $1 \mathrm{~V}$. dahliae strain JR2, similar to Ve1-transgenic plants (Figure 4A, B). In contrast, StuVe2- and StoVe2-transgenic plants were as diseased as non-transgenic controls (Figure 4A, B). Importantly, all genotypes were equally susceptible to the $V$. dahliae Ave1 deletion mutant (Figure 4A, B), suggesting that all these Ve1 alleles recognize the Ave1 effector. The phenotypes correlated with the degree of $V$. dahliae colonization as determined by real-time PCR (Figure 4). 


\section{Chapter 4}

Collectively, these data confirm that StuVe1 and StoVe1, but not StuVe2 and StoVe2, act as functional homologues of tomato Ve1 that confer resistance to race $1 \mathrm{~V}$. dahliae.

A

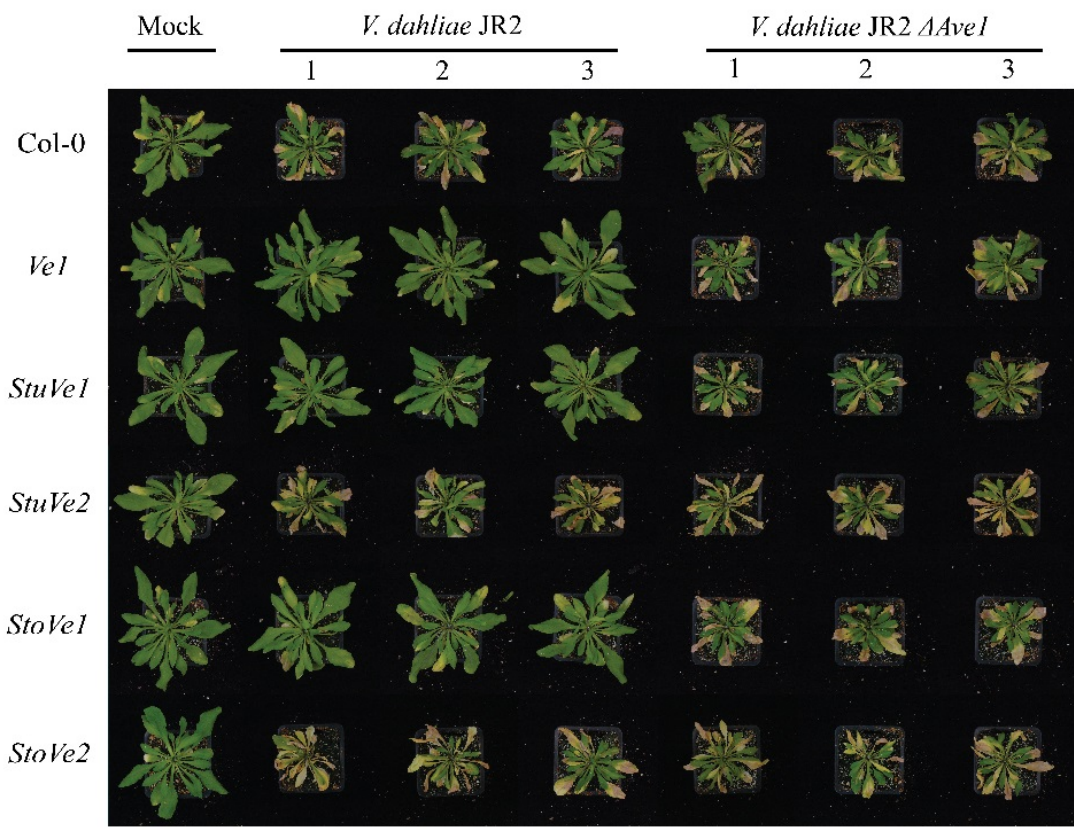

B

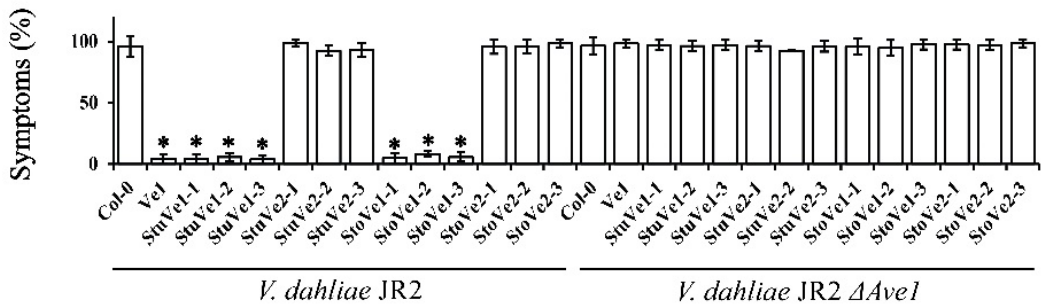

$\mathrm{C}$

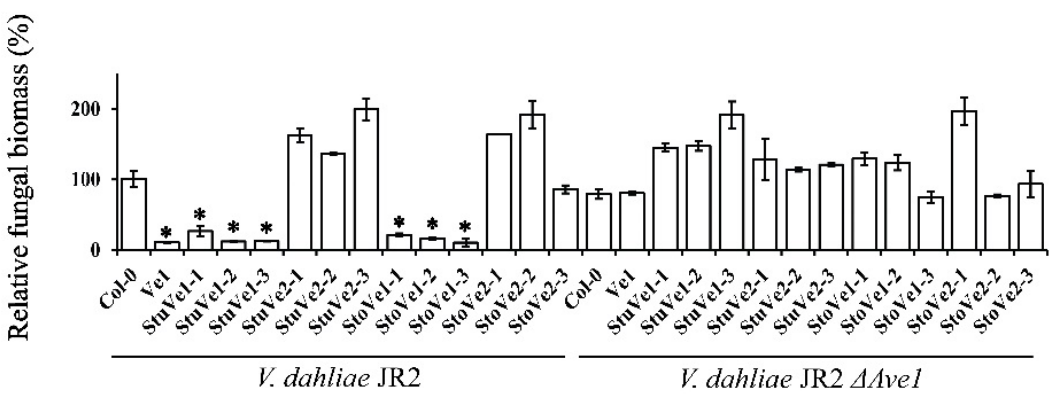


Figure 4. StuVe1 and StoVe1, but not StuVe2 and StoVe2, provide resistance against race 1 Verticillium dahliae in Arabidopsis. (A) Typical appearance of non-transgenic Arabidopsis and transgenic plants that engineered to express 35S-driven StuVe1, StuVe2, StoVe1 or StoVe2 upon mockinoculation or inoculation with $V$. dahliae JR2 or $V$. dahliae JR2 $\Delta$ Ave 1 at 21 days post inoculation (dpi). (B) Quantification of Verticillium wilt symptoms in Arabidopsis Col-0 and transgenic plants at 21 dpi. Bars represent quantification symptom development as percentage of diseased rosette leaves with standard deviation, with Col-0 (control) set to 100\%. (C) Fungal biomass determined by quantitative PCR (qPCR) in Arabidopsis Col-0 and transgenic plants at 21 dpi. Bars represent Verticillium internal transcribed spacer (ITS) transcript levels relative to AtRuBisCo (RuBisCo, ribulose-1,5-bisphosphate carboxylase/oxygenase) transcript levels (for equilibration) with standard deviation in a sample of five pooled plants. The fungal biomass in Col-0 (control) is set to $100 \%$. Three independent lines are shown (1, 2 and 3). Asterisks indicate significant differences when compared with Col-0 $(P<0.05)$. Ve1 transgenic plants were used as a positive control. The data shown are representative of at least three independent experiments.

\section{HLVe1-2A, but not HLVe1-2B, recognizes Verticillium effector Ave1}

Polygenic resistance to Verticillium spp. has also been described in several nonsolanaceous species, including hop, alfalfa, cotton and strawberry (Antanaviciute et al., 2015; Bolek et al., 2005; Jakse et al., 2013; Wang et al., 2008; Yang et al., 2008). Genetic resistance against Verticillium wilt in hop (Humulus lupulus) was introduced into breeding programs from American wild hop (H. lupulus var. neomexicanus) and is still used today as the main resistance source (Darby, 2001). Genetic analysis identified a single significant QTL for this resistance, suggesting that Verticillium wilt resistance in hop is conferred by more than a single gene (Jakse et al., 2013; Majer et al., 2014). To investigate the presence of Ve-like sequences in hop, Southern blotting with the tomato Ve1 gene as probe was performed, revealing low copy numbers of $V e$-like sequences in hop cultivars (Figure S5 and Methods S1). With thermal asymmetric interlaced (TAIL)-PCR (Terauchi and Kahl, 2000), several Ve-like sequences were identified (Methods S1). Further analysis revealed two Ve1 alleles in the Verticillium-resistant hop cultivar 'Wye Target', designated HLVe1-2A (GenBank accession: KJ647426) and HLVe1-2B (GenBank accession: KJ647427), which both encode 1039-amino-acid proteins (GenBank accessions: AIE39594 and AIE39595) sharing 52\% and 51\% identity with tomato Ve1 and Ve2, respectively, and 98\% identity with each other (Figure S1). To investigate the functionality of HLVe1-2A and HLVe1-2B, co-agroinfiltration with Ave1 in $N$. tabacum was performed. When mature tobacco leaves were coinfiltrated with a 1:1 mixture of A. tumefaciens cultures carrying Ave1 and HLVe1-2A, 


\section{Chapter 4}

signs of a weak HR were observed at 5 days post infiltration with a minor part of the infiltrated region developing necrosis (Figure 5A). However, in contrast, coexpression of $A v e 1$ and $H L V e 1-2 B$ in tobacco induced no such response, similar to co-agroinfiltration of Ve1, HLVe1-2A, and HLVe1-2B with Avr9 (Figure 5A). To test whether failure of HLVe1-2B to induce an HR is the result of the instability of the protein, the coding regions of $H L V e 1-2 A$ and $H L V e 1-2 B$ were cloned to generate Cterminally GFP-tagged expression constructs, and the stability of both proteins was verified by immunoblotting (Figure 5B).

A

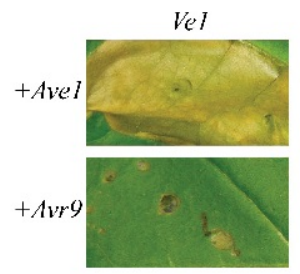

HLVel-2A

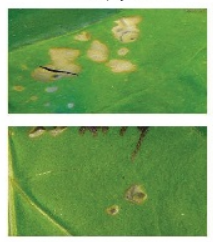

HLVe I-2B

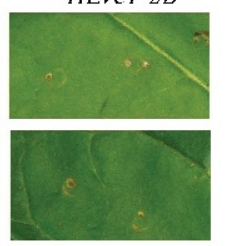

B

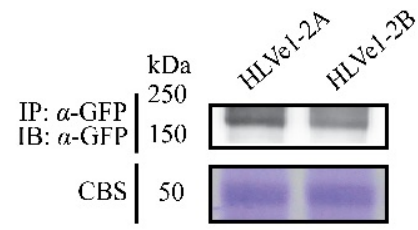

Figure 5. Co-expression of HLVe1-2A, but not HLVe1-2B, with Ave1 in Nicotiana tabacum activates a hypersensitive response (HR). (A) HLVe1-2A or HLVe1-2B was transiently co-expressed with Ave1 in N. tabacum, respectively. As a negative control, Avr 9 was co-expressed with $V e 1$ homologues. As a positive control, HR was induced upon co-expression of Ve1 and Ave1. (B) Green fluorescent protein (GFP)tagged HLVe1-2A and HLVe1-2B proteins are detected in planta by immunopurification (IP) using GFPbeads, followed by immunoblotting (IB) using $\alpha$-GFP antibody. Coomassie Blue Staining (CBS) of the blotting containing total protein extracts showed equal loading in each lane based on the $50-\mathrm{kDa}$ RuBisCo (ribulose-1,5-bisphosphate carboxylase/oxygenase) band.

To further assess the role of $H L V e 1-2 A$ and $H L V e 1-2 B$ in resistance to $V$. dahliae, heterologous expression in Arabidopsis was pursued (Figure S4). No phenotypic alterations were observed in plants that expressed HLVe1-2A or HLVe1-2B when compared with Ve1-transgenic or non-transgenic plants (Figure 6A), and three independent transgenic lines for $H L V e 1-2 A$ and $H L V e 1-2 B$ were assayed for $V$. dahliae resistance. Interestingly, despite the weak $\mathrm{HR}$ observed upon agroinfiltration together with Ave1 in N. tabacum, HLVe1-2A-expressing plants were clearly resistant to $V$. dahliae race 1 strain JR2 as few, if any, symptoms were observed (Figure 6A, B). In contrast, HLVe1-2B transgenic plants were as susceptible as non-transgenic controls (Figure 6A, B), and all genotypes were 
A

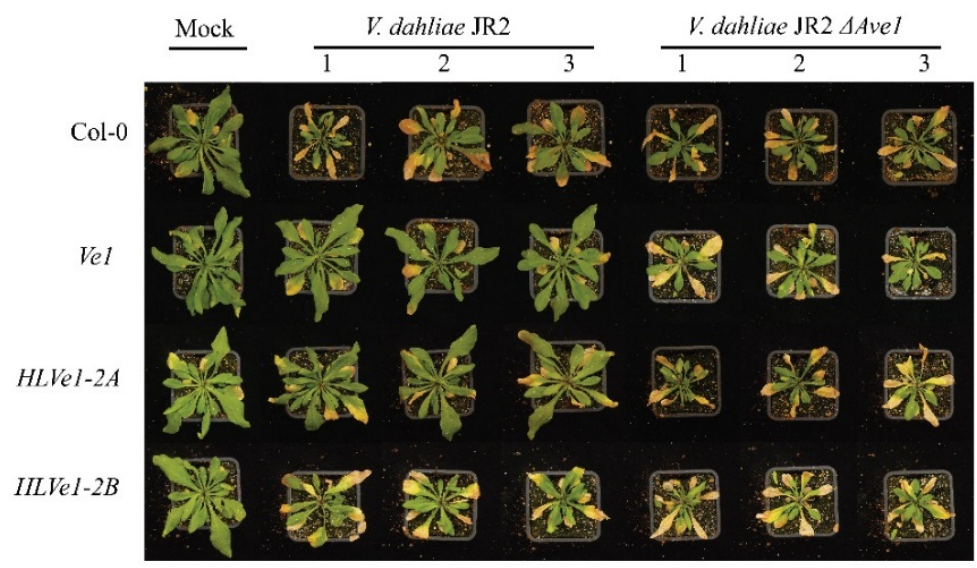

B

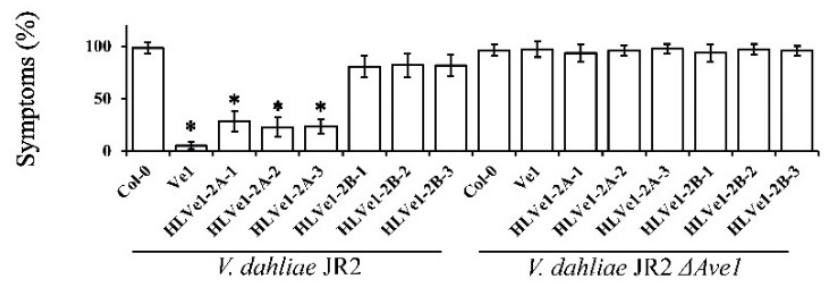

C

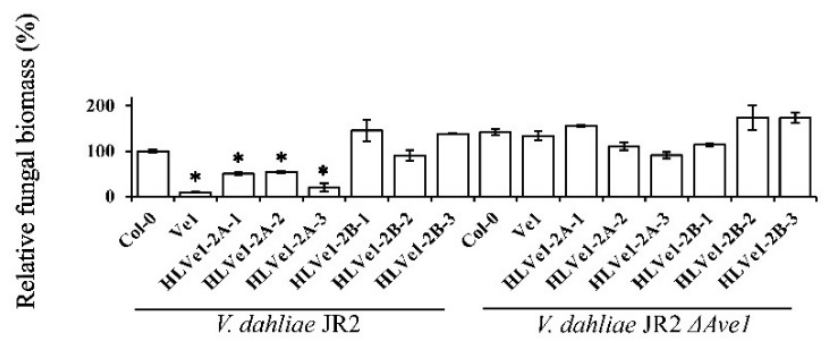

Figure 6. HLVe1-2A, but not HLVe1-2B, confers resistance to race 1 Verticillium dahliae in Arabidopsis. (A) Typical appearance of non-transgenic Arabidopsis and transgenic lines that constitutively express HLVe1-2A or HLVe1-2B upon mock-inoculation or inoculation with V. dahliae strain JR2 or $V$. dahliae JR2 $\triangle A v e 1$ at 21 days post inoculation (dpi). (B) Quantification of Verticillium wilt symptoms in Arabidopsis Col-0 and transgenic lines at $21 \mathrm{dpi}$. Bars represent quantification symptom development as percentage of diseased rosette leaves with standard deviation, with Col-0 (control) set to 100\%. (C) Fungal biomass determined by quantitative PCR (qPCR) in Arabidopsis Col-0 and transgenic plants at 21 dpi. Bars represent Verticillium internal transcribed spacer (ITS) transcript levels relative to AtRuBisCo (RuBisCo, ribulose-1,5-bisphosphate carboxylase/oxygenase) transcript levels (for equilibration) with standard deviation in a sample of five pooled plants. The fungal biomass in Col-0 (control) is set to $100 \%$. Three independent lines are shown (1, 2 and 3). Asterisks indicate significant differences when compared with Col-0 $(P<0.05)$. Ve1 transgenic plants were used as a positive control. The data shown are representative of at least three independent experiments. 


\section{Chapter 4}

equally susceptible to the $V$. dahliae Ave1 deletion mutant (Figure 6A, B). The phenotypes correlated with the level of $V$. dahliae biomass as determined by realtime PCR (Figure 6). Collectively, these data verify that HLVe1-2A, but not HLVe1$2 \mathrm{~B}$, acts a functional Ve1 homologue that provides resistance to race $1 \mathrm{~V}$. dahliae.

\section{Functional Ve1 homologues mediate recognition of Ave1 homologues from multiple plant pathogens}

We have previously shown that tomato Ve1 recognizes not only $V$. dahliae and $V$. albo-atrum Ave1, but also homologues derived from F. oxysporum f. sp. lycopersici (FoAve1) and C. beticola (CbAve1) (de Jonge et al., 2012). To investigate whether the newly identified functional Ve1 homologs similarly recognize these Ave1 homologues, co-expression of the five functional Ve1 homologs with a series of Ave1 homologs [FoAve1, CbAve1, ChAve1, CoAve1 (Colletotrichum orbiculare; Gan et al, 2013) and XacPNP (Gottig et al., 2008)] was performed (Figure 7A). Co-expression of the effector Avr9 (van Kan et al., 1991; Van der Hoorn et al., 2000) in combination with the Ve1 homologues was used as a negative control. To compare the HR induced upon co-expression of Ave1 homologues and functional Ve1 homologues in tobacco, HR development was measured by quantification of the leaf area that developed necrosis at 5 days post infiltration (Figure 7B). Co-expression of Ve1 with FoAve1 and CbAve1, but not with ChAve1, CoAve1 and XacPNP, in N. tabacum resulted in HR (Figure 7). StuVe1 seems to recognize a wider panel of Ave1 homologues, as co-expression with Ave1, FoAve1, CbAve1, ChAve1, CoAve1 and XacPNP induced HR (Figure 7). For NgVe1 and StoVe1, co-infiltration with Ave1 and FoAve1 induced HR, whereas infiltration with CbAve1, ChAve1, CoAve1 and XacPNP failed to induce HR (Figure 7). Finally, the most narrow recognition spectrum is observed for HLVe1-2A that recognizes none of the Ave1 homologues apart from that from $V$. dahliae (Figure 7). These data demonstrate that the newly identified functional Ve1 homologues, similar to tomato Ve1, differentially recognize Ave1 homologues from different plant pathogens. 
A
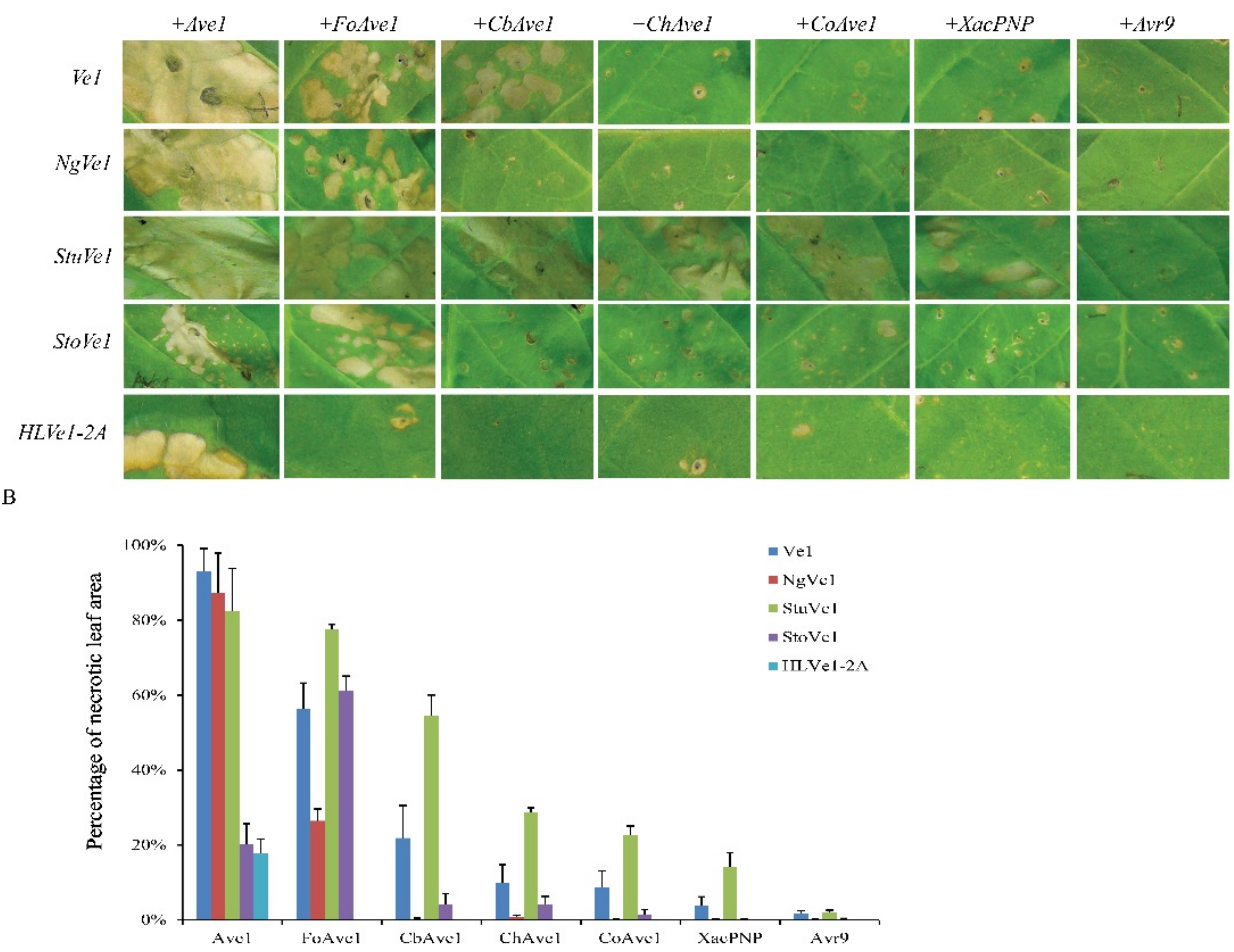

Figure 7. Distinct necrosis induced by Ave1 homologues through co-expression with functional Ve1 orthologues in Nicotiana tabacum. (A) Co-expression of functional Ve1 orthologues with Ave1 homologues (Ave1, FoAve1, CbAve1, ChAve1, CoAve1 and XacPNP) in N. tabacum. Expression of Avr9 in combination with $V e 1$ homologues is shown as negative controls. Leaves were photographed at 5 days post infiltration to visualize necrosis resulting from recognition by functional Ve1 homologues. (B) Quantification of necrosis resulting from recognition of Ave1 homologues by functional Ve1 orthologues at 5 days post infiltration. Bars represent the average percentage of necrotic leaf area of infiltration zones with standard deviation.

\section{Comparison of the protein sequences of Ve1 homologs}

Tomato Ve1 is predicted to contain a signal peptide, an eLRR domain composed by two eLRR regions, separated by a non-LRR island domain (also referred as C1, C3 and C2, respectively; Figure S6 and S7), a transmembrane domain, and a short cytoplasmic tail that lacks obvious motifs for intracellular signaling (Kawchuk et al., 2001; Wang et al., 2010; Zhang and Thomma, 2013). Alignment of the functional Ve1 protein sequences identified in this study clearly shows the typical eLRR-RLP domain architecture (Figure S6). All Ve1 homologues contain 37 eLRR repeats in two eLRR regions that are interrupted by a non-LRR island domain (Figure S6). 


\section{Chapter 4}

Previously, we have determined that three eLRR regions are crucial for Ve1 functionality; eLRR_1 to eLRR_8, eLRR_20 to eLRR_23 and eLRR_32 to eLRR_37 (Zhang et al., 2014). A comparison of the functional Ve1 homologues studied here shows that the eLRR_1 to eLRR_8 (44.2\% identity) and eLRR_20 to eLRR_23 (46.5\% identity) regions of the $\mathrm{C} 1$ domain are only slightly more conserved than the eLRR_9 to eLRR_19 (40.2\% identity) and eLRR_24 to eLRR_31 (45.0\% identity) regions of the $\mathrm{C} 1$ domain, respectively (Figure S6 and S7). A similar comparison of the C1 domains among the non-functional Ve1 homologues studied here shows that the eLRR_1 to eLRR_8 (50.2\% identity) region of the C1 domain is slightly more conserved that the eLRR_9 to eLRR_19 (45.5\% identity) region of the C1 domain, whereas the eLRR_20 to eLRR_23 (50.0\% identity) region of the C1 domain is conserved to a similar extent to the eLRR_24 to eLRR_31 (50.3\% identity) region of the C1 domain (Figure S7). Further comparison among the functional Ve1 homologues shows that the C3 domain (eLRR_32 to eLRR_37, 48.4\% identity) is more conserved than the $\mathrm{C} 1$ domain (43.3\% identity), C2 domain (8.0\% identity) and C-terminal eLRR-flanking domain (9.2\% identity) (Figure S6 and S7). This result is consistent with a previous comparison of tomato eLRR-RLPs, which showed that the C3 domain is more conserved than the C1 domain (Fradin et al., 2014). Finally, a comparison of the non-functional Ve1 homologues studied here shows that the C1 domain (48.7\% identity) and C3 domain (eLRR_32 to eLRR_37, $53.3 \%$ identity) are more conserved than the C2 domain (8.0\% identity) and Cterminal eLRR-flanking domain (7.2\% identity) (Figure S7). Collectively, these findings do not point towards a particular conservation of the three LRR regions that were previously implicated in Ve1 functionality.

\section{Phylogenetic analysis of Ve1 homologues in the plant kingdom}

To determine the phylogenetic breadth among Ve1 homologues in plants, we systematically queried the available genomes of 41 plant species for the occurrence of Ve1 homologues. In these genomes, we identified 1361 bona fide Ve1 homologues, all of which occur in land plants (embryophytes) and none in green algae (Figure 8A). To further analyze the phylogenetic relationship of tomato Ve1 and its close homologues, we used a neighbor-joining phylogeny to guide the extraction of the tomato Ve1 clade and the relevant sister clades (Figure 8B and S8). These 
sequences were used to infer a refined maximum likelihood phylogeny containing 608 Ve1 homologues that encompasses monocots and dicots. This phylogeny revealed a $V e 1$ orthologous group, defined at the last common ancestor of monocots and dicots, which contains all functional Ve1 homologues that have been described so far (Figure 8B and S8). The broad phylogenetic distribution, with homologues present in all land plants, establishes that $V e 1$ is an ancient immune receptor (Figure 8 and S8), and that the last common ancestor contained at least a single, but more likely several, Ve1-like genes. Moreover, we inferred a Ve1 orthologous group that comprises both monocots and dicots and includes all functional Ve1 genes, suggesting the conservation of function within this group of genes.

\section{DISCUSSION}

In this article, we describe the cloning and characterization of $V e 1$ homologues within and outside the Solanaceae family, and demonstrate that Ve1 homologues of tobacco (NgVe1), potato (StuVe1), wild eggplant (StoVe1) and hop (HLVe1-2A) act as functional homologues of tomato $\mathrm{Ve} 1$ by providing resistance to race $1 \mathrm{~V}$. dahliae strain, mediated through the recognition of the Ave1 effector, implying that functional Ve1 homologues are conserved across plant species within and outside the Solanaceae. We further show that all functional Ve1 proteins contain a conserved domain architecture with 37 eLRR repeats (Figure S6). It has been previously determined that the two regions of the C1 domain, namely eLRR_1 to eLRR_8 and eLRR_20 to eLRR-23, are required for Ve1 functionality, probably because they contribute to ligand binding (Zhang et al., 2014). Here, these regions appear to be only slightly more conserved than other regions within the functional Ve1 homologues (Figure S6 and S7). In addition, the C3 domain (eLRR_32 to eLRR_37) was shown to be critical for Ve1 functionality (Zhang et al., 2014; Figure S6), potentially through interaction with common factors required for downstream signalling (Fradin et al., 2014; Fritz-Laylin et al., 2005; Zhang and Thomma, 2013)). As expected, this region is most conserved among the functional Ve1 homologues (Figure S6 and S7). Previously, Ve1 homologues from other plant species have also been associated with Verticillium wilt resistance, although conclusive evidence for a causal role in resistance has mostly been lacking. For example, Vining and Davis (2009) showed that the mint Ve1 homologue $m V e 1$ associates with Verticillium wilt 
Chapter 4

A

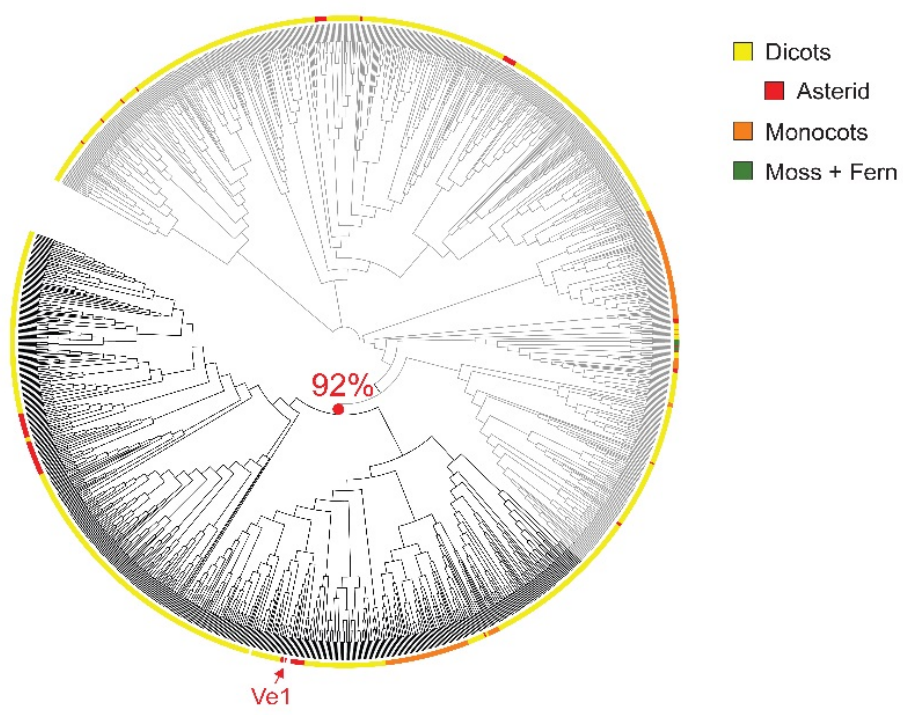

B

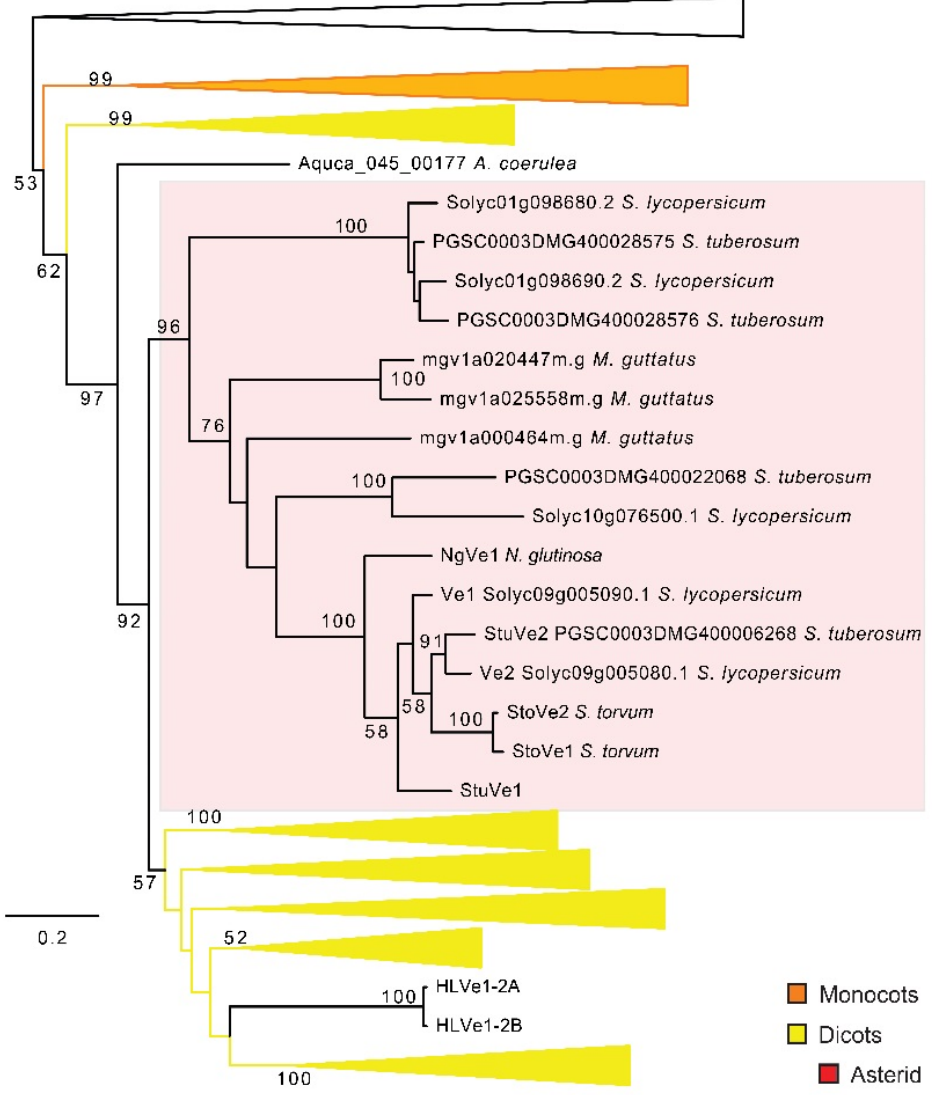


Figure 8. Phylogenetic analysis of Ve1 homologs indicates that Ve1 homologues are widely distributed in land plants. (A) Phylogeny of Ve1 proteins from 41 plant species from the Phytozome database. Tomato Ve1 is indicated with red arrow. (B) Maximum likelihood phylogenetic tree of selected Ve1 homologues (dark grey clades in A) displaying the tomato Ve1 clade and the relevant sister clades. Tomato Ve1 clade is indicated by highlighted background. Official gene identifiers and species name are indicated. Bootstrap values are shown in the tree. The scale represents branch length expressed as the relative number of amino acid substitutions.

resistance. Genome-wide analysis of disease resistance genes in lettuce, in combination with QTL mapping, showed that three Ve homologues, including Vr1, are located within a 100-kb region on chromosome 9 that co-segregates with resistance to race $1 \mathrm{~V}$. dahliae ( Christopoulou et al., 2015; Hayes et al., 2011). The grapevine $V e 1$ homologue $V v V e$ has been shown to enhance defense against $V$. dahliae in $N$. benthamiana (Tang et al., 2016). Remarkably, cotton Ve1 homologues GbVe, Gbve1, Gbvdr5 and Gbvdr3 have been shown to confer Verticillium resistance upon ectopic expression in Arabidopsis or cotton (Chen et al., 2016; Yang et al., 2014; Zhang et al., 2011; Zhang et al., 2012), although this concerns V. dahliae isolates that do not carry the Ave1 effector gene, and thus Verticillium wilt resistance cannot be mediated by Ave1 recognition in these cases (Song et al., unpublished data).

Previously, we have noted the absence of correlation between Ave1-induced HR and resistance through Ve1, as treatment with Ave1 leads to HR in tomato and tobacco plants that express Ve1, but not in N. benthamiana or Arabidopsis, whereas Ve1-transgenic Arabidopsis shows Ave1-triggered resistance (de Jonge et al., 2012; Zhang et al., 2013a, b). Similarly, in the present study, we observed robust resistance mediated by the S. torvum and hop Ve1 homologues StoVe1 and HLVe1$2 A$, whereas only a weak HR is observed on co-expression of Ave1. These findings suggest that the HR may occur as a consequence of Ve1/Ave1-induced immune signaling under particular conditions, but is not required for $V$. dahliae resistance (Zhang et al., 2013b). This finding may also explain that we were unable to compromise $\mathrm{NgVe1-mediated} \mathrm{V}$. dahliae resistance on VIGS in N. glutinosa, but were able to compromise Ave1-mediated HR.

Phylogenetic analysis revealed that Ve1 homologues are widely distributed in phylogenetically distant plant species, implying an ancient origin of the Ve1 immune receptor. Nevertheless, this origin does not imply functionality in $V$. 


\section{Chapter 4}

dahliae resistance. The most obvious example is the close tomato homologue Ve2 which, despite its homology, does not act as a functional $V$. dahliae resistance gene. Similarly, in this study, we identified the non-functional Ve1 homologues StuVe2, StoVe2 and HLVe1-2B. The Ve locus as it occurs in tomato, with two homologues Ve1 and $V e 2$, appears to originate before speciation, as clustered Ve1 family members also appear in potato and wild eggplant. Furthermore, a functional study addressing Ave1 recognition in the genus Nicotiana only identified Ave1 recognition, and thus presence of a potentially functional Ve1 homologue, in the species N. glutinosa (Zhang et al., 2013a). Considering the extremely wide host range of $V$. dahliae, and the general occurrence of strains that carry Ave1, the question arises whether the ancient progenitor of the currently functional Ve1 orthologues already functioned as a $V$. dahliae resistance gene, and thus that several species/homologues lost the capacity to recognize Ave1 as a result of adverse effects associated with Ve1 functionality, or whether several species/homologues evolved the capacity to recognize Ave1 after the occurrence of speciation events. The latter hypothesis would imply that Ave1 recognition evolved multiple times in the plant kingdom through parallel evolution. Our present data do not allow verification or disqualification of either hypothesis.

Plants and animals employ germline-encoded pattern recognition receptors (PRRs) to detect broadly distributed microbe-associated molecular patterns (MAMPs) and to activate antimicrobial defense (Macho and Zipfel, 2014). We have previously noted that tomato Ve1 is an ancient pathogen receptor with traits of typical PRRs. This finding was based on the transferability of Ve1 across plant species and the observation that Ve1 resistance affected three fungal species; $V$. dahliae, V. albo-atrum and F. oxysporum (Fradin et al., 2011; de Jonge et al., 2012; Thomma et al., 2011). We have now demonstrated that members of the Ve1 gene family in N. glutinosa, S. tuberosum, S. torvum and H. lupulus encode receptors that recognize Ave1 and are able to mediate $V$. dahliae resistance in Arabidopsis. As our findings are based on stable expression in a heterologous host, we realize that definitive evidence for the role of the Ve1 homologues in disease resistance in the endogenous hosts from which the Ve1 homologues are derived needs to be provided through targeted gene deletion in these hosts, or stable expression in susceptible genotypes of these species. We also discovered that the functional Ve1 
homologues have divergent recognition specificities, suggesting that some of them recognize an even wider spectrum of plant pathogens than tomato Ve1 (Figure 7). Collectively, these findings mean that tomato Ve1 has traits of a typical race-specific resistance protein as well as of a typical PRR. Similarly, Arabidopsis RLP23 recognizes an epitope of Nep-like effector proteins (NLPs) that are widely distributed among bacteria, fungi and oomycetes (Gijzen and Nürnberger, 2006) to induce immune responses (Albert et al., 2015). Thus, it is becoming apparent that MAMP receptor systems are more dynamic than generally appreciated and are conditioned similar to prototypical resistance genes (Albert et al., 2015; Cook et al., 2015; Shibuya and Desaki, 2015). Findings like these have inspired the proposal of the "Invasion Model", which describes plant immunity as a surveillance system to detect invasion, in which host receptors, termed invasion pattern receptors (IPRs), detect either externally encoded or modified-self ligands that indicate invasion, termed invasion patterns (IPs) (Cook et al., 2015).

\section{MATERIALS AND METHODS}

\section{Plant growth conditions and manipulations}

Plants were grown at $21^{\circ} \mathrm{C} / 19^{\circ} \mathrm{C}$ during $16 \mathrm{~h} / 8 \mathrm{~h}$ light/dark photoperiods, respectively, in the climate chamber or the greenhouse with a relative humidity of $\sim 75 \%$, and $100 \mathrm{~W} \cdot \mathrm{m}^{-2}$ supplemental light when light intensity dropped below 150 $\mathrm{W} \cdot \mathrm{m}^{-2}$. Arabidopsis transformations were performed as described previously (Clough and Bent, 1998).

\section{Isolation of Ve1 homologues}

The isolation of Ve1 homologues from N. glutinosa, S tuberosum, S. torvum and $H$. lupulus is described in Methods S1 and Tables S2-S4.

\section{Binary over-expression constructs and transient expression in planta}

For NgVe1, StuVe1, StuVe2, StoVe1, StoVe2 HLVe1-2A and HLVe1-2B constructs, CDS regions were amplified from $N$. glutinosa, S. tuberosum and $S$. torvum cDNA, respectively, while the CDS regions of $H L V e 1-2 A$ and $H L V e 1-2 B$ were amplified from the corresponding plasmids (Table S1). The CDS fragments were cloned into pDONR207 by using Gateway ${ }^{\circledR}$ BP Clonase ${ }^{\circledR}$ II Enzyme Mix (Invitrogen, Carlsbad, 
USA). All pDONR207 clones were sequenced, and fragments were subsequently transferred to pEarleyGate 100, pSol2095 (C-terminal GFP tag) (Zhang et al., 2013a), or to pFAST_R02 as described (Shimada et al., 2010) by using Gateway ${ }^{\circledR}$ LR Clonase $^{\circledR}$ II Enzyme Mix (Invitrogen, Carlsbad, USA). Constructs for constitutive expression of Ave1, FoAve1, CbAve1, ChAve1 were described previously (de Jonge et al., 2012). CoAve1 (Gan et al., 2013) and XacPNP (Gottig et al., 2008) were obtained by gene synthesis (Eurofins MWG Operon, Ebersberg, Germany), and subsequently recombined into the destination vector pSol2092 (Zhang et al., 2013a) to generate expression constructs pSol2092_CoAve1 and pSol2092_XacPNP. All constructs were transformed to Agrobacterium tumefaciens strain GV3101 (pMP90) by electroporation.

A. tumefaciens carrying expression constructs were infiltrated into tobacco plants as described previously (Zhang et al., 2013a). A. tumefaciens cultures containing constructs to express Ave1 and Ve1 homologues were mixed in a 1:1 ratio and infiltrated into leaves of 5- to 6-week-old tobacco plants. At 5 days post infiltration, pictures were taken, and necrosis was quantified by using ImageJ to measure the area of necrosis as percentage of the total infiltrated leaf area.

\section{Protein extracts and immunoblotting}

For detection of GFP-tagged Ve1 homologs, A. tumefaciens carrying the corresponding expression constructs was infiltrated into mature tobacco leaves as described previously (Zhang et al., 2013a). The co-immunopurifications and immunoblotting were performed as described previously (Zhang et al., 2014).

\section{Reverse transcription-PCR (RT-PCR) and reverse transcription-quantitative PCR (RT-qPCR)}

Target specificity of the constructs TRV::GUS and TRV::NgVe1 was determined in the TRV-infected N. glutinosa plants. Four weeks after TRV inoculation, whole tobacco plants were collected, and frozen in liquid nitrogen, and stored at $-80^{\circ} \mathrm{C}$ for total RNA isolation.

For the expression of $V e 1$ homologues in the corresponding transgenic plants, 2-week-old Arabidopsis seedlings were harvested and ground into a powder in liquid nitrogen. Total RNA extraction, cDNA synthesis and RT-PCR were performed 
as described earlier (Zhang et al., 2013b; Table S1). To analyse expression of $\mathrm{NgVe1}$ expression in TRV-targeted $N$. glutinosa plants, RT-qPCR was conducted using primers NgVe1-F(qRT) and NgVe1-R(qRT) with tobacco Actin as an endogenous gene (Table S1) using the qPCR Core Kit for SYBR Green I (Eurogentec Nederland BV, Maastricht, NL) as described earlier (Fradin et al., 2009).

\section{VIGS}

Constructs $p$ TRV2::PDS and pTRV2::GUS were used as controls. To silence NgVe1 in N. glutinosa, the construct $p T R V 2:: N g V e 1$ was generated. TRV vectors were agroinfiltrated as described before (Liu et al., 2002; Zhang et al., 2013a). Briefly, cotyledons of 10 - to 15 -day-old $N$. glutinosa seedlings were infiltrated with 1:1 mixtures of $p T R V 1$ and $p T R V 2$ constructs. Photobleaching was observed at 4 weeks after agroinfiltration of $p T R V 2:: P D S$. For HR assays, 4 weeks after virus inoculations, mature leaves were agroinfiltrated to individually express Ave1, VdNLP1 (Santhanam et al., 2013) and Avr9 (van Kan et al., 1991; Van der Hoorn et al., 2000). VdNLP1 and Avr9 were used as positive and negative controls, respectively. Five days after agroinfiltration, leaves were examined for the development of HR. For Verticillium wilt disease assays, 3 weeks after TRV infection, the TRV-infected plants were inoculated with race $1 \mathrm{~V}$. dahliae strain JR2 (Faino et al., 2015), and the corresponding Ave1 deletion mutant ( $V$. dahliae JR2 $\Delta$ Ave1; de Jonge et al., 2012) and tap water as control. The inoculated plants were evaluated by the observation of disease symptoms at $14 \mathrm{dpi}$.

\section{Disease assays}

Verticillium dahliae was grown on potato dextrose agar (PDA; Oxoid, Basingstoke, UK) at $22^{\circ} \mathrm{C}$, and conidia were collected from 7 - to 10 -day-old plates and washed with tap water. Verticillium disease assays on N. glutinosa plants were performed as described previously (Zhang et al., 2013a). Briefly, 5-week-old plants were uprooted, the roots were rinsed in water, dipped for $3 \mathrm{~min}$ in a suspension of $10^{6}$ conidiospores/mL water, and transferred to commercial potting soil (Horticoop, Bleiswijk, the Netherlands). Verticillium wilt disease assays on Arabidopsis, as well as Verticillium biomass quantification in infected Arabidopsis plants were performed as described before (Ellendorff et al., 2009). 


\section{Chapter 4}

\section{Phylogenetic identification}

To obtain the phylogenetic relationship of tomato Ve1 and its homologues, we identified similar sequences in 41 plant species acquired from Phytozome (v9.1) (Goodstein et al., 2012) and manually added Ve1 homologues of tobacco, potato, wild eggplant and hop. Sequence similarity was established using BLAST (version $322.28+$ ), applying a conservative e-value cutoff of $1 \mathrm{e}-50$. To prevent spurious hits, we removed sequences where the matching area was $<75 \%$ or the 'actualmatching' was $<50 \%$ of either Ve1 or the subject. The matching area is defined as the area from the start position of the first segment to the end position of the last segment, and the 'actual-matching' area is defined as the sum of the covered area by each individual segment. Moreover, sequences that deviate in length $(<80 \%$ or $>$ $120 \%$ of the length of Ve1) or contain protein domains other than leucine-rich repeats, as predicted by HMMER3 (version 3.0)(Finn et al., 2011) on a local PFAM database (version 27), were discarded. Subsequently, all protein sequences were aligned using MAFFT (version 7.047b) (Katoh et al., 2002) and the most consistent alignment (LINSI) was chosen using trimAl (version 1.2) (Capella-Gutiérrez et al., 2009), after which the heuristic method of trimAl was applied to trim the alignment. This cleaned alignment was used to construct an initial phylogenetic tree using quick tree (version 1.1; 1000 bootstraps). The clade of interest (with tomato Ve1) and surrounding sequences were manually gathered and realigned. The final phylogenetic tree was inferred using RAxML (version 7.6.3) (Stamatakis, 2006), with the gamma model of rate heterogeneity and the Whelan and Goldman amino acid substitution matrix.

\section{ACKNOWLEDGEMENTS}

Y.S. acknowledges a PhD fellowship from the China Scholarship Council. B.P.H.J.T. and M.F.S. are supported by a Vici and a Veni grant, respectively, of the Research Council for Earth and Life sciences (ALW) of the Netherlands Organization for Scientific Research (NWO). J.B. and J.J. acknowledge financial support from the Slovenian Research Agency, Grant Number P4-0077, and the support of A.M. by PhD grant (No.1000-09-310205). Bert Essenstam is acknowledged for excellent plant care at Unifarm. 


\section{REFERENCES}

Albert I, Böhm H, Albert M, Feiler CE, Imkampe J, Wallmeroth N, Brancato C, Raaymakers TM, Oome S, Zhang H, Krol E, Grefen C, Gust AA, Chai J, Hedrich R, Van den Ackerveken G, Nürnberger T (2015) An RLP23-SOBIR1-BAK1 complex mediates NLP-triggered immunity. Nat Plants 1: 15140.

Antanaviciute L, Šurbanovski N, Harrison N, McLeary K, Simpson D, Wilson F, Sargent D, Harrison R (2015) Mapping QTL associated with Verticillium dahliae resistance in the cultivated strawberry (Fragaria $\times$ ananassa). Hortic Res 2: 15009.

Bolek Y, El-Zik KM, Pepper AE, Bell AA, Magill CW, Thaxton PM, Reddy OUK (2005) Mapping of verticillium wilt resistance genes in cotton. Plant Sci 168: 1581-1590.

Boller T, Felix G (2009) A renaissance of elicitors: perception of microbe-associated molecular patterns and danger signals by pattern-recognition receptors. Annu Rev Plant Biol 60: 379-406.

Capella-Gutiérrez S, Silla-Martínez JM, Gabaldón T (2009) trimAl: a tool for automated alignment trimming in large-scale phylogenetic analyses. Bioinformatics 25: 1972-1973.

Chai Y, Zhao L, Liao Z, Sun X, Zuo K, Zhang L, Wang S, Tang K (2003) Molecular cloning of a potential Verticillium dahliae resistance gene SlVe1 with multi-site polyadenylation from Solanum licopersicoides. DNA Seq 14: 375-384.

Chen T, Kan J, Yang Y, Ling X, Chang Y, Zhang B (2016) A Ve homologous gene from Gossypium barbadense, Gbvdr3, enhances the defense response against Verticillium dahliae. Plant Physiol Biochem 98: 101-111.

Christopoulou M, Wo SR-C, Kozik A, McHale LK, Truco M-J, Wroblewski T, Michelmore R (2015) Genome-wide architecture of disease resistance genes in gettuce. G3 5: 2655-2699.

Clough SJ, Bent AF (1998) Floral dip: a simplified method for Agrobacterium-mediated transformation of Arabidopsis thaliana. Plant J 16: 735-743.

Cook DE, Mesarich CH, Thomma BPHJ (2015) Understanding plant immunity as a surveillance system to detect invasion. Annu Rev Phytopathol 53: 541-563.

Darby P (2001) "Single gene traits in hop breeding". Proceedings of the Scientific Commission of the International Hop Growers Convention, Canterbury, UK, (Seigner E, ed.) 76-80.

de Jonge R, van Esse HP, Maruthachalam K, Bolton MD, Santhanam P, Saber MK, Zhang Z, Usami T, Lievens B, Subbarao KV, Thomma BPHJ (2012) Tomato immune receptor Ve1 recognizes effector of multiple fungal pathogens uncovered by genome and RNA sequencing. Proc Natl Acad Sci USA 109: $5110-5115$.

Diwan N, Fluhr R, Eshed Y, Zamir D, Tanksley S (1999) Mapping of Ve in tomato: a gene conferring resistance to the broad-spectrum pathogen, Verticillium dahliae race 1. Theor Appl Genet 98: 315319.

Ellendorff U, Fradin EF, De Jonge R, Thomma BPHJ (2009) RNA silencing is required for Arabidopsis defence against Verticillium wilt disease. J Exp Bot 60: 591-602.

Faino L, Seidl MF, Datema E, van den Berg GC, Janssen A, Wittenberg AH, Thomma BPHJ (2015) Single-molecule real-time sequencing combined with optical mapping yields completely finished fungal genome. mBio 6: e00936-15.

Fei J, Chai Y, Wang J, Lin J, Sun X, Sun C, Zuo K, Tang K (2004) cDNA cloning and characterization of the Ve homologue gene StVe from Solanum torvum Swartz. DNA Seq 15: 88-95.

Fradin EF, Abd-El-Haliem A, Masini L, van den Berg GC, Joosten MH, Thomma BPHJ (2011) Interfamily transfer of tomato Ve1 mediates Verticillium resistance in Arabidopsis. Plant Physiol 156: $2255-2265$. 
Fradin EF, Thomma BPHJ (2006) Physiology and molecular aspects of Verticillium wilt diseases caused by V. dahliae and V. albo-atrum. Mol Plant Pathol 7: 71-86.

Finn RD, Clements J, Eddy SR (2011) HMMER web server: interactive sequence similarity searching. Nucleic Acids Res 39: W29-W37.

Fradin EF, Abd-El-Haliem A, Masini L, van den Berg GC, Joosten MH, Thomma BPHJ (2011) Interfamily transfer of tomato Ve1 mediates Verticillium resistance in Arabidopsis. Plant Physiol 156: $2255-2265$.

Fradin EF, Zhang Z, Ayala JCJ, Castroverde CD, Nazar RN, Robb J, Liu C-M, Thomma BPHJ (2009) Genetic dissection of Verticillium wilt resistance mediated by tomato Ve1. Plant Physiol 150: 320332.

Fradin EF, Zhang Z, Rovenich H, Song Y, Liebrand TW, Masini L, van den Berg GC, Joosten MH, Thomma BPHJ (2014) Functional analysis of the tomato immune receptor Ve1 through domain swaps with its non-functional homolog Ve2. PLOS ONE 9: e88208.

Fritz-Laylin LK, Krishnamurthy N, Tör M, Sjölander KV, Jones JD (2005) Phylogenomic analysis of the receptor-like proteins of rice and Arabidopsis. Plant Physiol 138: 611-623.

Gan P, Ikeda K, Irieda H, Narusaka M, O'Connell RJ, Narusaka Y, Takano Y, Kubo Y, Shirasu K (2013) Comparative genomic and transcriptomic analyses reveal the hemibiotrophic stage shift of Colletotrichum fungi. New Phytol 197: 1236-1249.

Gijzen M, Nürnberger T (2006) Nep1-like proteins from plant pathogens: recruitment and diversification of the NPP1 domain across taxa. Phytochemistry 67: 1800-1807.

Goodstein DM, Shu S, Howson R, Neupane R, Hayes RD, Fazo J, Mitros T, Dirks W, Hellsten U, Putnam N (2012) Phytozome: a comparative platform for green plant genomics. Nucleic Acids Res 40: D1178-D1186.

Gottig N, Garavaglia BS, Daurelio LD, Valentine A, Gehring C, Orellano EG, Ottado J (2008) Xanthomonas axonopodis pv. citri uses a plant natriuretic peptide-like protein to modify host homeostasis. Proc Natl Acad Sci USA 105: 18631-18636.

Hayes RJ, McHale LK, Vallad GE, Truco MJ, Michelmore RW, Klosterman SJ, Maruthachalam K, Subbarao KV (2011) The inheritance of resistance to Verticillium wilt caused by race 1 isolates of Verticillium dahliae in the lettuce cultivar La Brillante. Theor Appl Genet 123: 509-517.

Inderbitzin P, Davis RM, Bostock RM, Subbarao KV (2011) The ascomycete Verticillium longisporum is a hybrid and a plant pathogen with an expanded host range. PLOS ONE 6: e18260.

Jakse J, Cerenak A, Radisek S, Satovic Z, Luthar Z, Javornik B (2013) Identification of quantitative trait loci for resistance to Verticillium wilt and yield parameters in hop (Humulus lupulus L.). Theoretical and applied genetics 126: 1431-1443

Katoh K, Misawa K, Kuma Ki, Miyata T (2002) MAFFT: a novel method for rapid multiple sequence alignment based on fast Fourier transform. Nucleic Acids Res 30: 3059-3066.

Kawchuk LM, Hachey J, Lynch DR, Kulcsar F, van Rooijen G, Waterer DR, Robertson A, Kokko E, Byers R, Howard RJ (2001) Tomato Ve disease resistance genes encode cell surface-like receptors. Proc Natl Acad Sci USA 98: 6511-6515.

Liu SP, Zhu YP, Xie C, Jue DW, Hong YB, Chen M, Hubdar AK, Yang Q (2012) Transgenic potato plants expressing StoVe1 exhibit enhanced resistance to Verticillium dahliae. Plant Mol Biol Rep 30: 10321039.

Liu Y, Schiff M, Dinesh-Kumar S (2002) Virus-induced gene silencing in tomato. Plant J 31: 777-786.

Macho AP, Zipfel C (2014) Plant PRRs and the activation of innate immune signaling. Mol Cell 54: 263272.

Majer A, Javornik B, Cerenak A, Jakse J (2014) Development of novel EST-derived resistance gene 
markers in hop (Humulus lupulus L.). Mol Breed 33: 61-74.

Santhanam P, van Esse HP, Albert I, Faino L, Nürnberger T, Thomma BPHJ (2013) Evidence for functional diversification within a fungal NEP1-like protein family. Mol Plant-Microbe Interact 26: 278-286.

Senthil-Kumar M, Hema R, Anand A, Kang L, Udayakumar M, Mysore KS (2007) A systematic study to determine the extent of gene silencing in Nicotiana benthamiana and other Solanaceae species when heterologous gene sequences are used for virus-induced gene silencing. New Phytol 176: 782-791.

Shibuya N, Desaki Y (2015) Immunity: One receptor, many pathogens. Nat Plants 1: 15149.

Shimada TL, Shimada T, Hara-Nishimura I (2010) A rapid and non-destructive screenable marker, FAST, for identifying transformed seeds of Arabidopsis thaliana. Plant J 61: 519-528.

Simko I, Costanzo S, Haynes K, Christ B, Jones R (2004a) Linkage disequilibrium mapping of a Verticillium dahliae resistance quantitative trait locus in tetraploid potato (Solanum tuberosum) through a candidate gene approach. Theor Appl Genet 108: 217-224.

Simko I, Haynes K, Ewing E, Costanzo S, Christ B, Jones R (2004b) Mapping genes for resistance to Verticillium albo-atrum in tetraploid and diploid potato populations using haplotype association tests and genetic linkage analysis. Mol Genet Genomics 271: 522-531.

Stamatakis A (2006) RAxML-VI-HPC: maximum likelihood-based phylogenetic analyses with thousands of taxa and mixed models. Bioinformatics 22: 2688-2690.

Tang J, Lin J, Yang Y, Chen T, Ling X, Zhang B, Chang Y (2016) Ectopic expression of a Ve homolog VvVe gene from Vitis vinifera enhances defense response to Verticillium dahliae infection in tobacco. Gene 576: 492-498.

Terauchi R, Kahl G (2000) Rapid isolation of promoter sequences by TAIL-PCR: the 5'-flanking regions of Pal and Pgi genes from yams (Dioscorea). Mol Gen Genetics 263: 554-560.

Thomma BPHJ, Nürnberger T, Joosten MH (2011) Of PAMPs and effectors: the blurred PTI-ETI dichotomy. Plant Cell 23: 4-15.

Van der Hoorn RA, Laurent F, Roth R, De Wit PJ (2000) Agroinfiltration is a versatile tool that facilitates comparative analyses of Avr 9/Cf-9-induced and Avr 4/Cf-4-induced necrosis. Mol PlantMicrobe Interact 13: 439-446.

van Kan JA, Van den Ackerveken G, De Wit PJ (1991) Cloning and characterization of cDNA of avirulence gene avr9 of the fungal pathogen Cladosporium fulvum, causal agent of tomato leaf mold. Mol. Plant-Microbe Interact 4: 52-59.

Vining K, Davis T (2009) Isolation of a Ve homolog, $m V e 1$, and its relationship to verticillium wilt resistance in Mentha longifolia (L.) Huds. Mol Genet Genomics 282: 173-184.

Wang G, Ellendorff U, Kemp B, Mansfield JW, Forsyth A, Mitchell K, Bastas K, Liu C-M, Woods-Tör A, Zipfel C, De Wit PJ, Jones JDG, Thomma BPHJ (2008) A genome-wide functional investigation into the roles of receptor-like proteins in Arabidopsis. Plant Physiol 147: 503-517.

Wang G, Fiers M, Ellendorff U, Wang Z, de Wit PJ, Angenent GC, Thomma BP (2010) The diverse roles of extracellular leucine-rich repeat-containing receptor-like proteins in plants. Crit Rev Plant Sci 29: 285-299.

Wang HM, Lin ZX, Zhang XL, Chen W, Guo XP, Nie YC, Li YH (2008) Mapping and quantitative trait loci analysis of Verticillium wilt resistance genes in cotton. J Integr Plant Biol 50: 174-182.

Xu X, Pan S, Cheng S, Zhang B, Mu D, Ni P, Zhang G, Yang S, Li R, Wang J, et al. (2011) Genome sequence and analysis of the tuber crop potato. Nature 475: 189-195.

Yang C, Guo W, Li G, Gao F, Lin S, Zhang T (2008) QTLs mapping for Verticillium wilt resistance at seedling and maturity stages in Gossypium barbadense L. Plant Sci 174: 290-298 


\section{Chapter 4}

Yang Y, Ling X, Chen T, Cai L, Liu T, Wang J, Fan X, Ren Y, Yuan H, Zhu W, Zhang B, Ma DP (2014) A cotton Gbvdr5 gene encoding a leucine-rich repeat receptor-like protein confers resistance to Verticillium dahliae in transgenic Arabidopsis and upland cotton. Plant Mol Biol Rep 33: 1-15.

Zhang B, Yang Y, Chen T, Yu W, Liu T, Li H, Fan X, Ren Y, Shen D, Liu L, Liu L, Dou D, Chang Y (2012) Island cotton Gbve1 gene encoding a receptor-like protein confers resistance to both defoliating and non-defoliating isolates of Verticillium dahliae. PLOS ONE 7: e51091.

Zhang Y, Wang X, Yang S, Chi J, Zhang G, Ma Z (2011) Cloning and characterization of a Verticillium wilt resistance gene from Gossypium barbadense and functional analysis in Arabidopsis thaliana. Plant Cell Rep 30: 2085-2096.

Zhang Z, Fradin E, de Jonge R, van Esse HP, Smit P, Liu CM, Thomma BPHJ (2013a) Optimized agroinfiltration and virus-induced gene silencing to study Ve1-mediated Verticillium resistance in tobacco. Mol Plant Microbe Interact 26: 182-190.

Zhang Z, Esse HP, Damme M, Fradin EF, Liu CM, Thomma BPHJ (2013b) Ve1-mediated resistance against Verticillium does not involve a hypersensitive response in Arabidopsis. Mol Plant Pathol 14: 719-727.

Zhang Z, Song Y, Liu CM, Thomma BPHJ (2014) Mutational analysis of the Ve1 immune receptor that mediates Verticillium resistance in tomato. PLOS ONE 9: e99511.

Zhang Z, Thomma BPHJ (2013) Structure-function aspects of extracellular leucine-rich repeatcontaining cell surface receptors in plants. J Integr Plant Biol 55: 1212-1223. 


\section{SUPPLEMENTAL INFORMATION}

\begin{tabular}{l|c|c|c|c|c|c|c|c}
\hline & Vel & NgVel & StuVel & StuVe2 & StoVe1 & StoVe2 & HLVe1-2ム & HLVe1-2B \\
\hline Ve1 & - & $76 \%$ & $87 \%$ & $84 \%$ & $83 \%$ & $80 \%$ & $52 \%$ & $51 \%$ \\
Ve2 & $84 \%$ & $74 \%$ & $81 \%$ & $91 \%$ & $81 \%$ & $85 \%$ & $52 \%$ & $51 \%$ \\
NgVe1 & $76 \%$ & - & $78 \%$ & $74 \%$ & $74 \%$ & $73 \%$ & $50 \%$ & $50 \%$ \\
StuVe1 & $87 \%$ & $78 \%$ & - & $82 \%$ & $82 \%$ & $81 \%$ & $51 \%$ & $51 \%$ \\
StuVe2 & $84 \%$ & $74 \%$ & $82 \%$ & - & $81 \%$ & $85 \%$ & $51 \%$ & $51 \%$ \\
StoVe1 & $83 \%$ & $74 \%$ & $82 \%$ & $81 \%$ & - & $92 \%$ & $51 \%$ & $51 \%$ \\
StoVe2 & $80 \%$ & $73 \%$ & $81 \%$ & $85 \%$ & $92 \%$ & - & $51 \%$ & $51 \%$ \\
HIVe1-2A & $52 \%$ & $50 \%$ & $51 \%$ & $51 \%$ & $51 \%$ & $51 \%$ & - & $98 \%$ \\
IlLVel-2B & $51 \%$ & $50 \%$ & $51 \%$ & $51 \%$ & $51 \%$ & $51 \%$ & $98 \%$ & - \\
\hline
\end{tabular}

Figure S1. Percentage of amino acid identity shared between nine Ve1 homologues. The highest percentage of homology between two Ve1 homologues is indicated in red. Dashes (-) represent identical sequences.
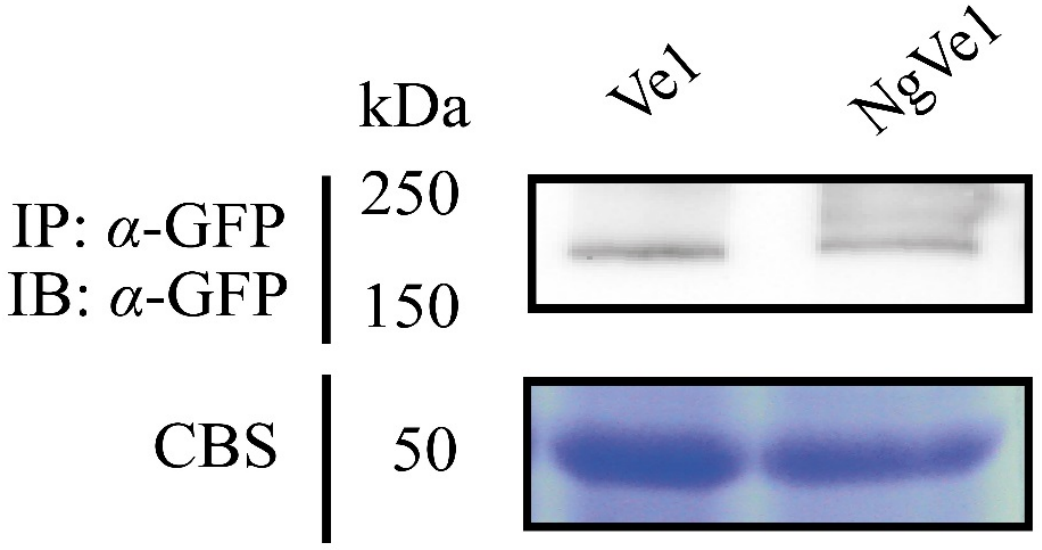

Figure S2. Stability of green fluorescent protein (GFP)-fused NgVe1 protein in planta. Total protein extracts of transiently transformed leaf tissue were subjected to immunopurification (IP) by using GFPaffinity beads. Immunopurified proteins were subjected to sodium dodecylsulfate-polyacrylamide gel electrophoresis (SDS/PAGE) and immunoblotted (IB) using $\alpha$-GFP antibody. Coomassie Blue Staining (CBS) of the blot containing total protein extracts showed equal loading in each lane based on the $50 \mathrm{kDa}$ RuBisCo (ribulose-1,5-bisphospate carboxylase/oxygenase) band. GFP-tagged Ve1 protein was used as a control. 
$\Lambda$

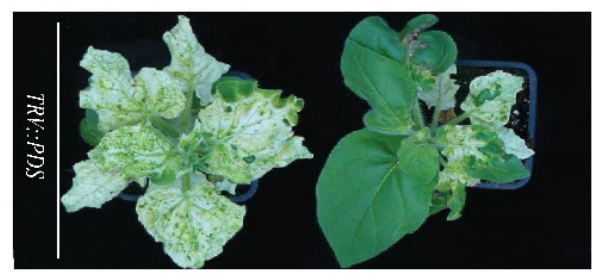

B

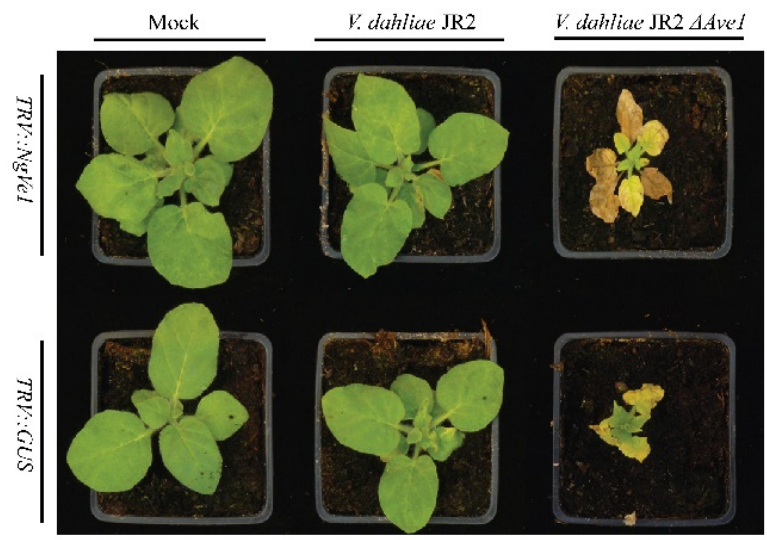

$\mathrm{C}$

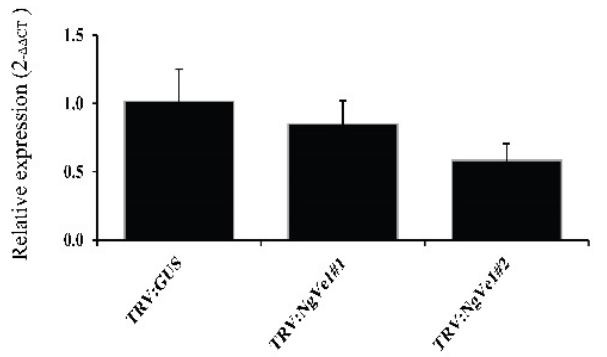

Figure S3. Tobacco rattle virus (TRV)-mediated gene silencing in Nicotiana glutinosa. (A) Virusinduced gene silencing of the phytoene desaturase $(P D S)$ gene results in patchy photobleaching in leaves of $N$. glutinosa. Pictures were taken at 4 weeks post TRV::PDS infiltration, and show representative infected plants of at least three independent assays. (B) TRV::NgVe1-inoculated plants show resistance against Verticillium dahliae strain JR2, but not V. dahliae JR2 $\Delta$ Ave1. N. glutinosa plants were inoculated with a recombinant TRV targeting the $\beta$-glucuronidase (GUS) gene as a control (TRV::GUS) or recombinant TRV targeting the $\mathrm{NgVe1}$ gene (TRV::NgVe1). At 3 weeks after TRV infiltration, TRVinoculated plants were inoculated either with $V$. dahliae strain JR2, or with $V$. dahliae JR2 $\Delta A v e 1$ mutant. Pictures were taken at 2 weeks after $V$. dahliae inoculation, and show representative inoculated plants of at least three independent assays. (C) Silencing efficiency was determined by using reverse transcription-quantitative PCR (RT-qPCR) at 28 days post infiltration in TRV::NgVe1- and TRV::GUSinoculated N. glutinosa plants. Bars represent levels of $\mathrm{NgVe1}$ transcripts relative to the transcript levels of $N$. glutinosa actin (for normalization) with standard deviation of a sample of three pooled plants. NgVe1 expression in the TRV::GUS-infected plants is set to 1. 


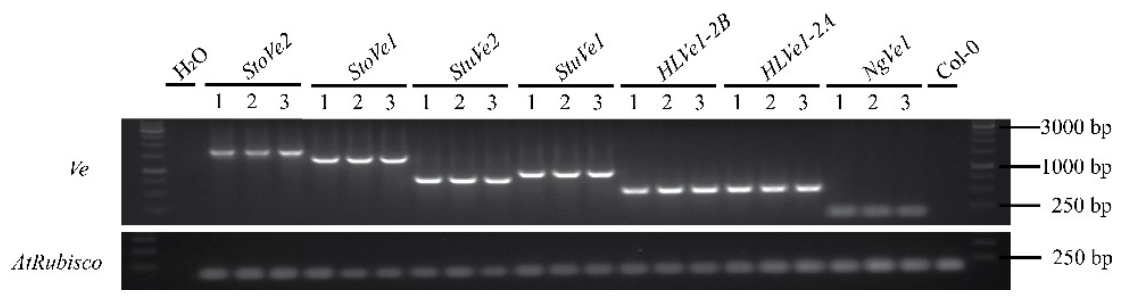

Figure S4. Expression of $\mathrm{NgVe1}, \mathrm{HLVe1-2A}, \mathrm{HLVe1-2B}$, StuVe1, StuVe2, StoVe1 and StoVe2 in transgenic plants was detected by reverse transcription-PCR (RT-PCR). As an endogenous control, a fragment of the AtRuBisCo (RuBisCo, ribulose-1,5-bisphospate carboxylase/oxygenase) gene was amplified from cDNA. For each construct, three independent transgenic lines are shown (1, 2 and 3). Water is used as a control.
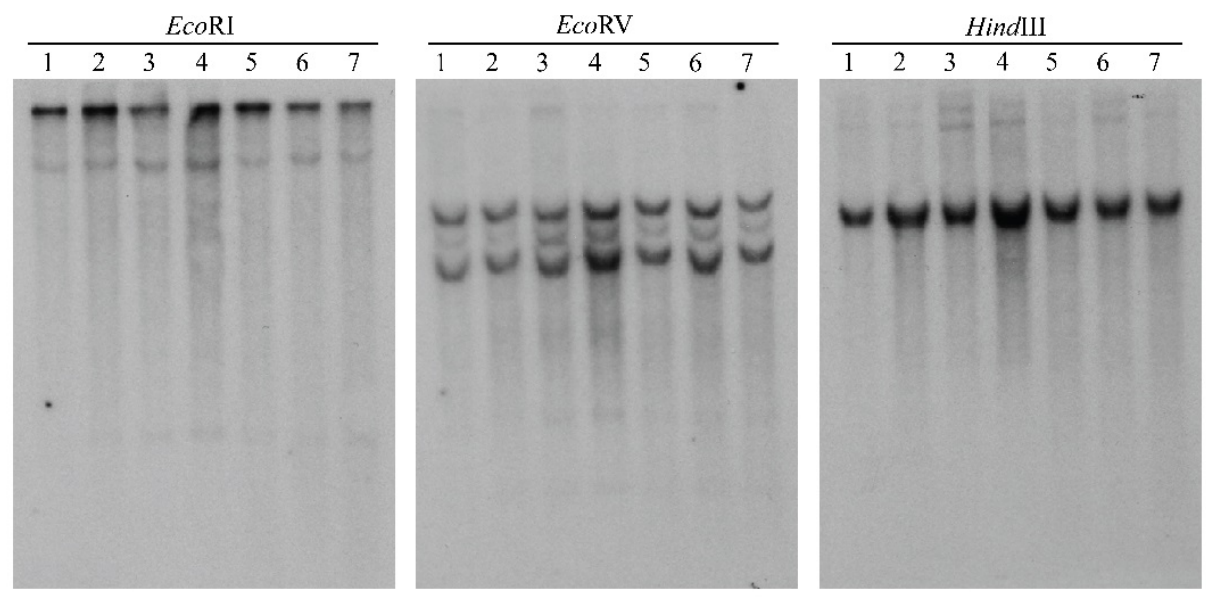

Figure S5. Sothern blotting of seven hop cultivars with the tomato Ve1 gene as a probe reveals the presence of $V e$-like sequences in the hop genome. Seven hop cultivars are indicated as: 1, 'Wye target'; 2, 'Fuggle'; 3, 'Wye Challanger'; 4, 'Savinjski Golding'; 5, 'Aurora'; 6, 'Celeia'; 7, 'Yeoman'. Hop genomic DNA was digested with the restriction enzymes EcoRI, EcoRV and HindIII, separated on 0.8\% agarose gel and blotted on nylon membranes with the $\mathrm{P}^{32}$-labelled tomato Ve1 gene sequence as a probe. 


\section{Chapter 4}
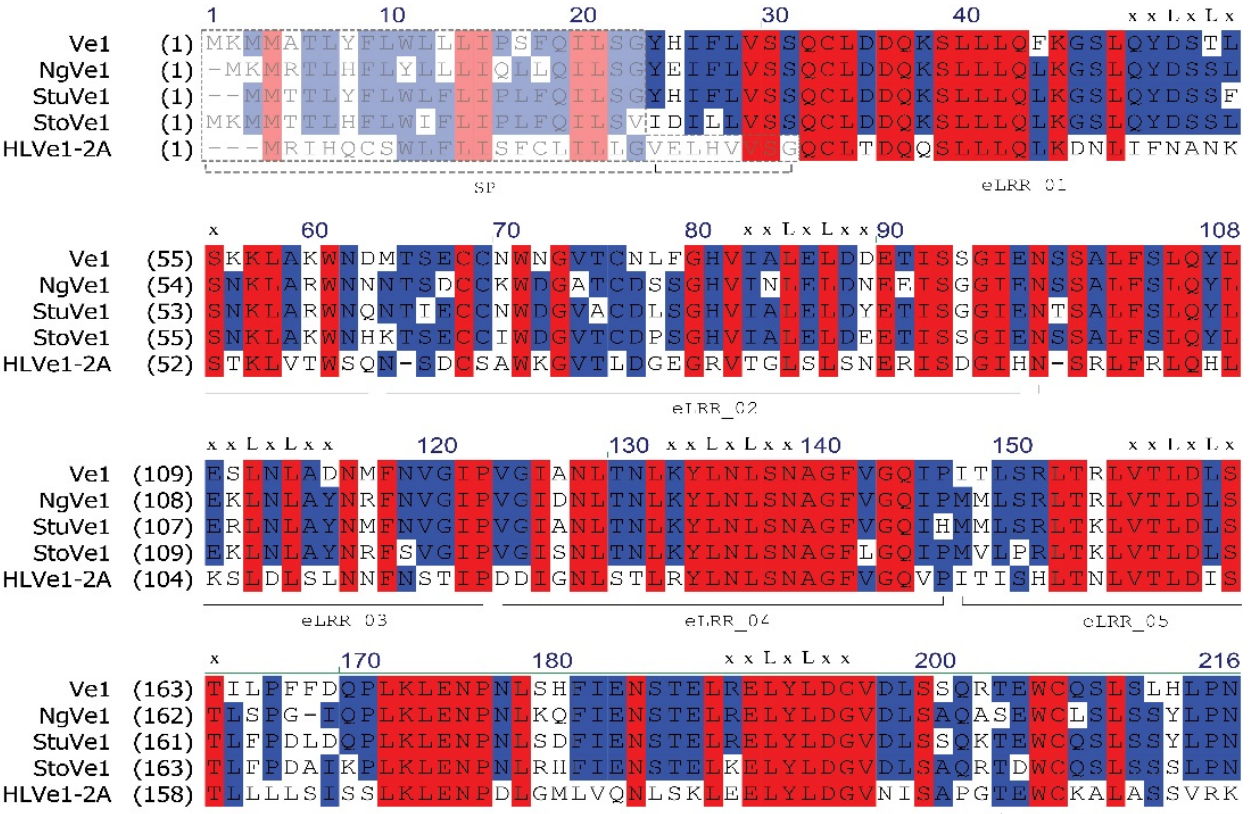

eLkR 06
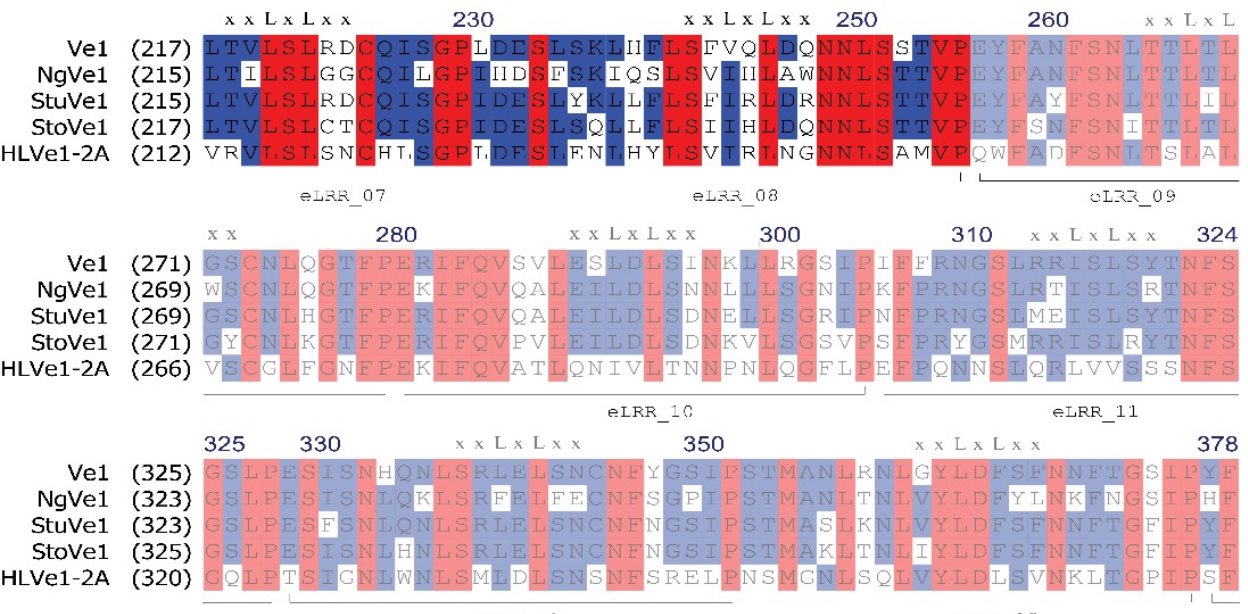

$379 \times \times 1 . \times 1 \times \times \frac{\text { OLRR_12 }}{400}$

eLRR 13

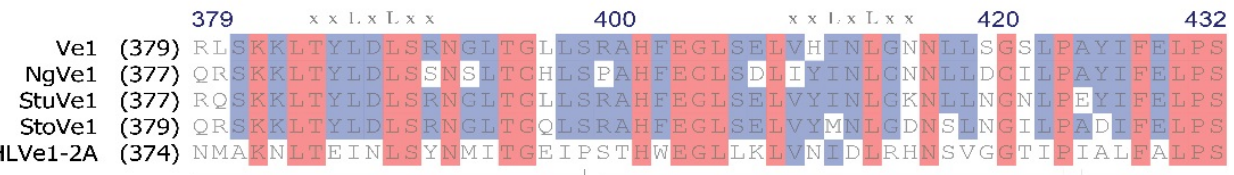

HLVe1-2A (374) NMAN LIEINISYNMITGEIPSTHWEGLLRLVNIDLRHNSVGGTIEIALEALPS

$$
\text { CLRR_14 ELRR } \div 5
$$

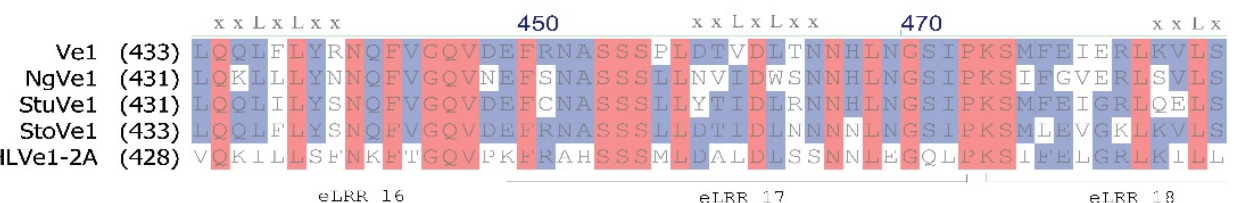



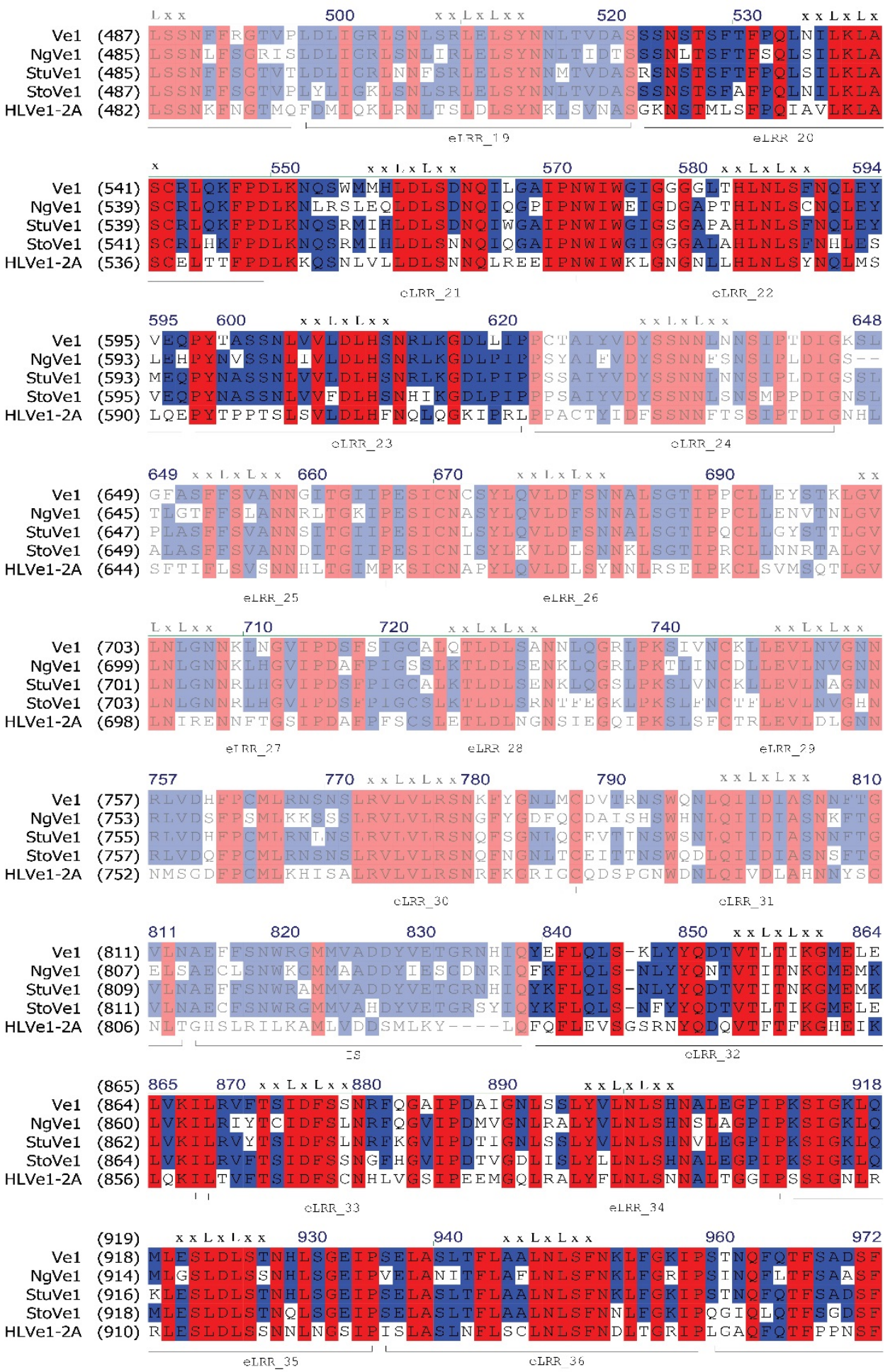


\section{Chapter 4}

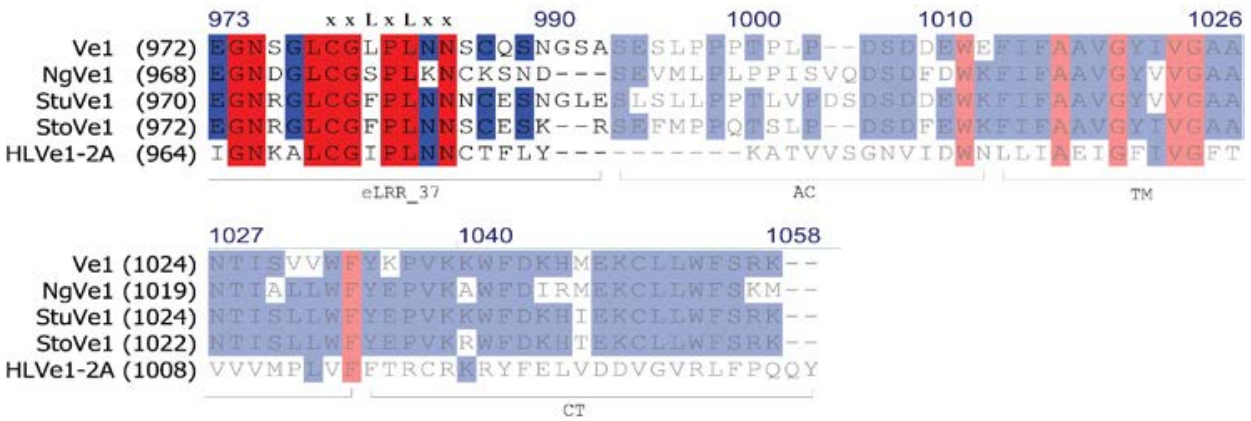

Figure S6. Primary structure and protein sequence alignment of functional Ve1 proteins from tomato, tobacco, potato, wild eggplant and hop. The $\mathrm{N}$-terminal amino acids in the dashed frame denote the predicted signal peptides (SPs) of the functional Ve1 homologues. eLRR, extracelluar leucinerich repeat (C1 domain and C3 domain); IS, non-LRR island domain (C2 domain); AC, acidic domain; TM, transmembrane domain; CT, cytoplasmic domain. The locations of the predicted solvent-exposed $\beta$ sheet (xxLxLxx) on the concave surface of the receptor are indicated above the eLRR domains. Identical amino acid residues are highlighted in red, whereas conserved amino acid residues are highlighted in blue. Three consecutive eLRR regions required for the functionality of Ve1 are indicated by bold colors, whereas other regions that could not be implicated in Ve1 functionality are indicated in light colors. Ve1 homologue sequences can be found in the GenBank database using the following GenBank accessions: ACR33105 (Ve1); ALK26499 (NgVe1); ALK26501 (StuVe1); ALK26500 (StoVe1); AIE39594 (HLVe1-2A). (A high resolution image of the Figure S6 can be downloaded from the link: http://onlinelibrary.wiley.com/doi/10.1111/mpp.12390/full).

\begin{tabular}{|c|c|c|c|c|}
\hline \multirow{8}{*}{$\begin{array}{l}\text { Functional Vel } \\
\text { homologs }\end{array}$} & \multicolumn{2}{|c|}{ Protein sequence region } & \multicolumn{2}{|c|}{ Protein sequence identity } \\
\hline & \multirow{4}{*}{$\begin{array}{l}\text { C1 domain } \\
\text { (eLRR_1 to eLRR_31) }\end{array}$} & eLRR_1 to eLRR_8 & \multirow{4}{*}{$43.3 \%$} & $44.2 \%$ \\
\hline & & eLRR_9 to eLRR_19 & & $40.2 \%$ \\
\hline & & eLRR 20 to eLRR 23 & & $46.5 \%$ \\
\hline & & eLRR_24 to eLRR_31 & & $45.0 \%$ \\
\hline & \multicolumn{2}{|l|}{$\mathrm{C} 2$ domain } & \multicolumn{2}{|c|}{$8.0 \%$} \\
\hline & \multicolumn{2}{|c|}{ C3 domain (eLRR_32 to eLRR_37) } & \multicolumn{2}{|c|}{$48.4 \%$} \\
\hline & \multicolumn{2}{|c|}{ C-terminal eLRR-flanking domain } & \multicolumn{2}{|c|}{$9.2 \%$} \\
\hline \multirow{7}{*}{$\begin{array}{l}\text { Non-functional } \\
\text { Vel homologs }\end{array}$} & \multirow{4}{*}{$\begin{array}{l}\text { Cl domain } \\
\text { (eLRR_1 to eLRR_31) }\end{array}$} & eLRR_1 to eLRR_8 & \multirow{4}{*}{$48.7 \%$} & $50.2 \%$ \\
\hline & & eLRR_9 to eLRR_19 & & $45.5 \%$ \\
\hline & & eLRR_20 to eLRR_23 & & $50.0 \%$ \\
\hline & & eLRR_24 to eLRR_31 & & $50.3 \%$ \\
\hline & \multicolumn{2}{|l|}{$\mathrm{C} 2$ domain } & \multicolumn{2}{|c|}{$8.0 \%$} \\
\hline & \multicolumn{2}{|c|}{ C3 domain (eLRR_32 to eLRR_37) } & \multicolumn{2}{|c|}{$53.3 \%$} \\
\hline & \multicolumn{2}{|c|}{ C-terminal eLRR-flanking domain } & \multicolumn{2}{|c|}{$7.2 \%$} \\
\hline
\end{tabular}

Figure S7. Percentage of amino acid identity shared between the C1 domain, C2 domain, C3 domain and C-terminal extracelluar leucine-rich repeat (eLRR)-flanking domain of the functional and non-functional Ve1 homologues from tomato, tobacco, potato, wild eggplant and hop. 


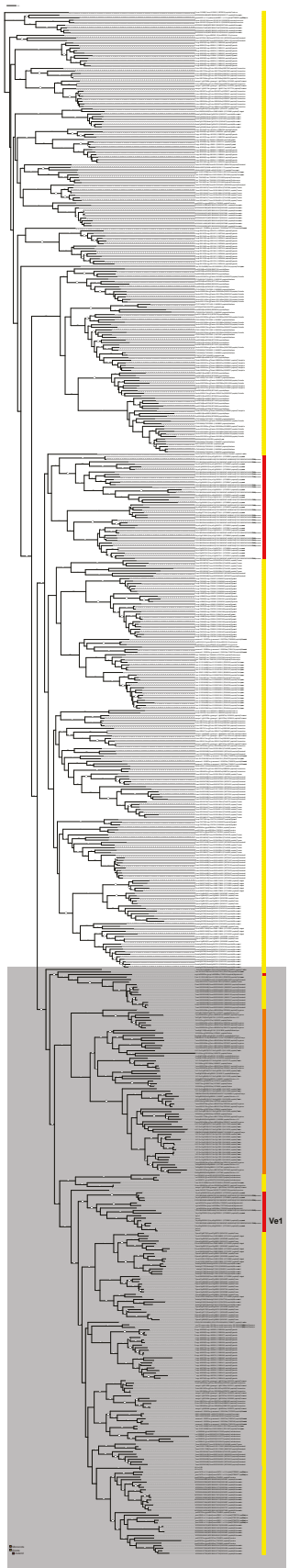

Figure S8. An unrooted phylogenetic tree based on protein sequences of Ve1 homologues from the tomato clade and the relevant sister clades. The Tomato Ve1 clade is indicated by the highlighted background. Official gene identifiers and species name are indicated. Bootstrap values are shown in the tree. The scale represents branch length expressed as the relative number of amino acid substitutions.

(A high resolution image of the Figure S8 can be downloaded from the link: http://onlinelibrary.wiley.com/doi/10.1111/mpp.12390/full). 


\section{Chapter 4}

\section{Methods S1. Isolation of Ve1 homologues}

A 1300 bp fragment was amplified from Nicotiana glutinosa cDNA using primers combination (Ve1SeqF1 and NgVe1-GSPR). To obtain the full-length $\mathrm{NgVe1}$ sequence, 5' and 3' rapid amplification of cDNA ends (RACE) reactions were performed by using the GeneRace ${ }^{\circledR}$ Core Kit (Invitrogen, Carlsbad, USA) following the manufacturer's instructions with minor modifications. Forward gene-specific primers NgVe1-GSPF1 and NgVe1-GSPF2 were used for 3' RACE, while reverse gene-specific primers NgVe1-GSPR1 and NgVe1-GSPR2 were used for 5' RACE (Table S1). The first round of PCR was followed by a nested PCR. The RACE products were purified and cloned into the Zero Blunt ${ }^{\circledR}{ }^{\text {TOPO }}{ }^{\circledR}$ PCR Cloning Kit (Invitrogen, Carlsbad, USA) and sequenced. The full-length $\mathrm{NgVe1}$ was determined by aligning 5' and 3' RACE product sequences with the partial cDNA fragment cloned earlier (Zhang et al., 2013). Primers NgVe1-F and NgVe1-R (Table S1) were designed based on sequence information of inserts from multiple clones derived from 5' RACE and 3' RACE products, and were utilized to amplify the full-length cDNA and genomic DNA (gDNA) from N. glutinosa. PCR reactions were performed using the Thermo Scientific Phusion High-Fidelity PCR Kit (Thermo Scientific, Massachusetts, USA). The amplicons of full-length NgVe1 from N. glutinosa cDNA and gDNA were sequenced using pre-mixed sequencing primers (Table S1). The sequence of full-length $\mathrm{NgVe1}$ is deposited at NCBI under accession number: KT895339.

To amplify the coding sequences (CDSs) of Ve gene homologues in heterozygous diploid potato, the primer pairs StuVe1-attB1/StuVe1-attB2 and StuVe2-attB1/StuVe2-attB2, respectively (Table S1), were designed based on the sequences of the Ve locus (NCBI reference sequence: XM_006362308 and XM_006362309) from a doubled monoploid potato genotype Phureja DM 1-3 516 R44 (Xu et al., 2011). To obtain the CDSs of StoVe1 and StoVe2, the primer pairs StoVe1-attB1/StoVe1-attB2 and StoVe2attB1/StoVe2-attB2, respectively (Table S1), were designed based on the sequence of StoVe1 (GenBank accession: DQ020574; Liu et al., 2012) and StVe (GenBank accessions: AY311527; Fei et al., 2004) from wild eggplant. All PCR products were cloned into pDONR207 by using Gateway ${ }^{\circledR}$ BP Clonase ${ }^{\circledR}$ II Enzyme Mix (Invitrogen, Carlsbad, USA), and subsequently sequenced using pre-mixed sequencing primers (Table S1), and named StuVe1 (GenBank accession: KT946795) and StuVe2 (GenBank accession: KT946797), StoVe1 (GenBank accession: KT946794) and StoVe2 (GenBank accession: KT946796).

Southern blotting was performed to query for Ve homologues in hop. $5 \mu \mathrm{g}$ of hop genomic DNA (seven hop cultivars: 'Wye target', 'Fuggle', 'Wye Challanger', 'Savinjski Golding', 'Aurora', 'Celeia' and 'Yeoman') was digested with EcoRI, EcoRV and HindIII, separated on 0.8\% agarose gel and transferred to nylon membranes. Southern blotting was performed with the $\mathrm{P}^{32}$-labelled tomato Ve1 gene sequence as a probe and washing was performed with 0.5X SSC (Maniatis et al., 1982). A pair of specific and degenerative primers HLVe(deg)-F and HLVe(deg)-R (Table S1) was constructed based on the protein 
ClustalW alignment of tomato Ve1 (GenBank accession: AAK58682), mint Verticillium wilt resistance-like protein (GenBank accession: ACB99689), three vine grape proteins (GenBank accessions: CA063881, CAN76702 and CA063885) and a hop EST sequence (GenBank accessions: GD247683), which showed similarity to Ve1. A Ve-like sequence from hop cultivar 'Wye Target' with a length of $\sim 1300$ bp was amplified. Its alignment showed similarity to Ve1 from amino acid 142 forward. This part of the sequence was the basis for isolation of the flanking sequences of HLVe using TAIL-PCR combined six degenerate primers (Table S2) with designed hop sequence-specific primers (Table S3) from the hop cultivar 'Wye target'. A primary TAIL-PCR reaction was performed in $20 \mu \mathrm{l}$ containing $20 \mathrm{ng}$ of hop genomic DNA, 1X Dream Taq Buffer, 0.8 mM of dNTPs, 0.8 U of Dream Taq enzyme (Thermo Scientific, Massachusetts, USA), $0.2 \mathrm{mM}$ of primary reaction hop specific primer and one of the following arbitrary primers: AD1, AD6 $(2 \mu \mathrm{M}), \operatorname{AD} 2(3 \mu \mathrm{M}), \operatorname{AD} 3, \operatorname{AD} 4$ or AD5 (4 $\mu \mathrm{M})$ (Table S2; S3). The reaction was diluted 50 times and $1 \mu \mathrm{l}$ was used in the secondary TAIL-PCR reaction $(20 \mu \mathrm{l})$ with a changed amount of Dream Taq polymerase $(0.6 \mathrm{U})$, secondary reaction hop specific primer $(0.2 \mathrm{mM})$ and a different amount of $\mathrm{AD}$ primers (AD1 and AD6 1,5 $\mu \mathrm{M}, \mathrm{AD} 2, \mathrm{AD} 3, \mathrm{AD} 4$ or AD5 $2 \mu \mathrm{M}$ ) (Table S2; S3). The reaction mixture of the tertiary TAIL-PCR was identical to the secondary one, with the exception that $1 \mu$ l of diluted secondary reaction diluted 1:50 was added as template and tertiary reaction hop specific primer was used (Table S2; S3). The amplification protocols are listed as Table S4. Amplicons of tertiary TAIL-PCR reactions were cleaned and sequenced and the obtained sequences were assembled in CodonCode Aligner (version 3.7.1); contigs were further checked for similarity with Ve1 using blast $\mathrm{X}$ algorithm. Based on the assembled sequences, two full-length Ve1-like sequences were amplified from hop cultivar 'Wye Target' genomic DNA using primers HLVe-F and HLVe-R (Table S1). Two sequences were deposited at NCBI as HLVe1-2A (GenBank accession: KJ647426) and HLVe1-2B (GenBank accession: KJ647427). 


\section{Chapter 4}

Table S1. Primers used for amplification, sequencing and expression of Ve1 homologs.

\begin{tabular}{|c|c|c|}
\hline Primer name & Oligonucleotide sequence $\left(5^{\prime} \rightarrow 3^{\prime}\right)$ & Description $^{a}$ \\
\hline Ve1SeqF1 & TTCAATGTTGGCATACCAGTTGG & Fradin et al., 2011 \\
\hline NgVe1-GSPR (Nested) & ACTTGCATTCCAACAGGCTTAAGGGT & 5' RACE \\
\hline GeneRacer $^{\mathrm{TM}} 3^{\prime}$ Primer & GCTGTCAACGATACGCTACGTAACG & $\begin{array}{l}\text { 3'RACE } \\
\text { (provided in RACE Kit) }\end{array}$ \\
\hline GeneRacer $^{\mathrm{TM}} 3^{\prime}$ Nested Primer & CGCTACGTAACGGCATGACAGTG & $\begin{array}{l}\text { 3'RACE } \\
\text { (provided in RACE Kit) }\end{array}$ \\
\hline GeneRacer $^{\mathrm{TM}} 5^{\prime}$ Primer & CGACTGGAGCACGAGGACACTGA & $\begin{array}{l}\text { 5'RACE } \\
\text { (provided RACE Kit) }\end{array}$ \\
\hline GeneRacer $^{\mathrm{TM}} 5^{\prime}$ Nested Primer & GGACACTGACATGGACTGAAGGAGTA & $\begin{array}{l}\text { 5'RACE } \\
\text { (provided in RACE Kit) }\end{array}$ \\
\hline NgVe1-GSPF1 & CAATGCCTTGAGTGGCACAATACCA & 3'RACE \\
\hline NgVe1-GSPF2 (Nested) & TCTTGGAGTGCTGAATCTTGGGAAC & 3'RACE \\
\hline NgVe1-GSPR1 & CCAAGCAAGATGGATGACAGAAAGAG & 5'RACE \\
\hline NgVe1-GSPR2 (Nested) & TCGCTCGCCTGAGCTGAAAGATCAA & 5'RACE \\
\hline NgVe1-F & GTCATATACCTATACAAGTTTGCATG & $\mathrm{NgVe1}$ transcription sequence \\
\hline NgVe1-R & GTACTCCTCATTTATTGGTTTAAGC & $\mathrm{NgVe} 1$ transcription sequence \\
\hline NgVe1-attB1 & ggggacaagtttgtacaaaaaagcaggctATGAAAATGAGAACTCTACA & $\mathrm{NgVe} 1$ coding sequence \\
\hline NgVe1-attB2 & ggggaccactttgtacaagaaagctgggtTCACATCTTTGAAAACCA & $\mathrm{NgVe} 1$ coding sequence \\
\hline $\mathrm{NgVe} 1-\operatorname{attB} 2(\Delta \mathrm{SC})$ & ggggaccactttgtacaagaaagctgggtcCATCTTTGAAAACCAAAG & GFP-tagged NgVe1 \\
\hline NgVe1-F(TRV) & ggggacaagtttgtacaaaaaagcaggctGACTCTCAGACCTCATCTACAT & NgVe1 (VIGS) \\
\hline NgVe1-R(TRV) & ggggaccactttgtacaagaaagctgggtCTGGGAAAAAGTGAATGAC & NgVe1 (VIGS) \\
\hline NgVe1-F(qRT) & TCTTTCTGTCATCCATCTTGC & NgVe1 (RT-qPCR \& RT-PCR) \\
\hline NgVe1-R(qRT) & TGTCTCCTGACTCAATGTAATC & NgVe1 (RT-qPCR \& RT-PCR) \\
\hline HLVe(deg)-F & ACGCCAGTAAATCGACGAAG & $\begin{array}{l}\text { Degenerative primer for hop } \\
V e \text {-like sequence }\end{array}$ \\
\hline HLVe(deg)-R & CKRTTISWRTGIARRTCIAHAVE & $\begin{array}{l}\text { Degenerative primer for hop } \\
V e \text {-like sequence }\end{array}$ \\
\hline HLVe-F & TTCGTTTGCCCATTTTGTTT & $\begin{array}{l}\text { Full length hop Ve1-like } \\
\text { sequence }\end{array}$ \\
\hline HLVe-R & TGAACGCCTTCTTGGTCT & $\begin{array}{l}\text { Full length hop Ve1-like } \\
\text { sequence }\end{array}$ \\
\hline HLVe1-attB1 & ggggacaagtttgtacaaaaaagcaggctATGAGAATTCATCAGTGT & $\begin{array}{l}H L V e 1-2 A \& H L V e 1-2 B \text { coding } \\
\text { sequence }\end{array}$ \\
\hline HLVe1-attB2 & ggggaccactttgtacaagaaagctgggtTTAATACTGTTGAGGAAA & $\begin{array}{l}\text { HLVe1-2A \& HLVe1-2B coding } \\
\text { sequence }\end{array}$ \\
\hline HLVe1-attB2 $(\Delta \mathrm{SC})$ & ggggaccactttgtacaagaaagctgggtcATACTGTTGAGGAAAGAG & $\begin{array}{l}\text { GFP-tagged HLVe1-2A \& } \\
\text { HLVe1-2B }\end{array}$ \\
\hline HLVe1-F & TTGCCTCGTGTGAACTAACTAC & $\begin{array}{l}\text { HLVe1-2A \& HLVe1-2B (Seq \& } \\
\text { RT-PCR) }\end{array}$ \\
\hline HLVe1-R & CCTGTGAAGTTGTTTTCTCGTA & $\begin{array}{l}\text { HLVe1-2A \& HLVe1-2B (Seq \& } \\
\text { RT-PCR) }\end{array}$ \\
\hline StuVe1-attB1 & ggggacaagtttgtacaaaaaagcaggctATGATGACAACTCTGTACTTTCT & StuVe1 coding sequence \\
\hline StuVe1-attB2 & ggggaccactttgtacaagaaagctgggtTCACTTTCTTGAAAACCAAA & StuVe1 coding sequence \\
\hline StuVe1-attB2 $(\Delta \mathrm{SC})$ & ggggaccactttgtacaagaaagctgggtaCTTTCTTGAAAACCAAAG & GFP-tagged StuVe1 \\
\hline
\end{tabular}




\section{Broad taxonomic characterization of Verticillium wilt resistance genes}

Table S1 (continued)

\begin{tabular}{|c|c|c|}
\hline Primer name & Oligonucleotide sequence $\left(5^{\prime} \rightarrow 3^{\prime}\right)$ & Description $^{\mathrm{a}}$ \\
\hline StuVe2-attB1 & ggggacaagtttgtacaaaaaagcaggctATGAGATTTTTACACTTTCTATGG & StuVe2 coding sequence \\
\hline StuVe2-attB2 & ggggaccactttgtacaagaaagctgggtTCAAAACTTTTTGTGATATATGAC & StuVe2 coding sequence \\
\hline StuVe2-attB2( $\Delta \mathrm{SC})$ & ggggaccactttgtacaagaaagctgggtaAAACTTTTTGTGATATATGACT & GFP-tagged StuVe2 \\
\hline StuVe1-F1(Seq) & CTTGTCAGATAACGAGTTGCTT & Potato StuVe1 (Seq) \\
\hline StuVe1-R1(Seq) & GAAATGAATCTGGGATAACACC & Potato StuVe1 (Seq) \\
\hline StuVe1-F2(Seq) & CTTTCACATTTCCCCAGTTG & Potato StuVe1 (seq) \\
\hline StuVe1-R2(Seq) & CGTAATCATCAGCAACCATC & Potato StuVe1 (Seq) \\
\hline StuVe2-F1(Seq) & TTCCACAATGGCAAACCTTA & Potato StuVe2 (Seq) \\
\hline StuVe2-R1(Seq) & CCTTCAGGCTGGTTGAGTTC & Potato StuVe2 (Seq) \\
\hline StuVe2-F2(Seq) & АCTTTCTCTCTTCCATCCGT & Potato StuVe2 (Seq) \\
\hline StuVe2-R2(Seq) & GAAGTTGGAAGAAAGTGAGAG & Potato StuVe2 (Seq) \\
\hline StoVe1-attB1 & ggggacaagtttgtacaaaaaagcaggctATGAAAATGATGACAACTCTCC & StoVe1 coding sequence \\
\hline StoVe1-attB2 & ggggaccactttgtacaagaaagctgggtTCACTTCCTTGAAAACCAAA & StoVe1 coding sequence \\
\hline StoVe1-attB2 $(\Delta \mathrm{SC})$ & ggggaccactttgtacaagaaagctgggtcCTTCCTTGAAAACCAAAGC & GFP-tagged StoVe1 \\
\hline StoVe2-attB1 & ggggacaagtttgtacaaaaaagcaggctATGAGATTTTTACACTTTCTATGG & StoVe2 coding sequence \\
\hline StoVe2-attB2 & ggggaccactttgtacaagaaagctgggtTCAAAACTTTTTGTGACATATGAC & StoVe2 coding sequence \\
\hline StoVe2-attB2 $(\Delta \mathrm{SC})$ & ggggaccactttgtacaagaaagctgggtcAAACTTTTTGTGACATATGACC & GFP-tagged StoVe2 \\
\hline StoVe1-F(Seq) & AGTGGTAGTGTTCCGAGTTT & Wild eggplant StoVe1 (Seq) \\
\hline StoVe1-R(Seq) & ACATTCAGGACCTCCAAAAA & Wild eggplant StoVe1 (Seq) \\
\hline StoVe2-F(Seq) & AAGTTGCTTAGTGGTAGTATTC & Wild eggplant StoVe2 (Seq) \\
\hline StoVe2-R(Seq) & TGGAGGAACTTATACTGGAT & Wild eggplant StoVe2 (Seq) \\
\hline M13-F & CGCCAGGGTTTTCCCAGTCACGAC & pCR blunt II TOPO (Seq) \\
\hline M13-R & TCACACAGGAAACAGCTATGAC & pCR blunt II TOPO (Seq) \\
\hline pENTRattL1 & TCGCGTTAACGCTAGCATGGATCTC & pDONR207 (Seq) \\
\hline pENTRattL2 & ACATCAGAGATTTTGAGACACGGGC & pDONR207 (Seq) \\
\hline NgActin-F & TATGGAAACATTGTGCTCAGTGG & Tobacco actin (RT-qPCR) \\
\hline NgActin-R & CCAGATTCGTCATACTCTGCC & Tobacco actin (RT-qPCR) \\
\hline AtRubisco-F & GCAAGTGTTGGGTTCAAAGCTGGTG & Arabidopsis Rubisco \\
\hline AtRubisco-R & CCAGGTTGAGGAGTTACTCGGAATGCTG & Arabidopsis Rubisco \\
\hline ITS1-F & AAAGTTTTAATGGTTCGCTAAGA & V. dahliae \\
\hline STVe1-R & CTTGGTCATTTAGAGGAAGTAA & V. dahliae \\
\hline
\end{tabular}

aThe type of experiment for which the primers were used is indicated in brackets (RACE: rapid amplification of cDNA ends, VIGS: Virusinduced gene silencing, Seq: sequencing, RT-PCR: Reverse transcription-PCR, RT-qPCR: reverse transcription-quantitative PCR). 


\section{Chapter 4}

Table S2. Degenerative primers used for TAIL-PCR amplification of the flanking regions of putative hop Ve1 homologues.

\begin{tabular}{|c|c|c|c|}
\hline Primer name & Oligonucleotide sequence $\left(5^{\prime} \rightarrow 3^{\prime}\right)$ & Level of degeneracy & Reference \\
\hline AD1 & NTCGASTWTSGWGTT & $64 X$ & Liu et al., 1995 \\
\hline AD2 & NGTCGASWGANAWGAA & $128 \mathrm{X}$ & Liu et al., 1995 \\
\hline AD3 & WGTGNAGWANCANAGA & $256 X$ & Liu et al., 1995 \\
\hline $\mathrm{AD} 4$ & STTGNTASTNCTNTGC & $256 \mathrm{X}$ & $\begin{array}{l}\text { Qiu et al., } 2010 \\
\text { (AD8) }\end{array}$ \\
\hline AD5 & WCAGNTGWTNGTNCTG & $256 \mathrm{X}$ & $\begin{array}{l}\text { Qiu et al., } 2010 \\
\text { (AD9) }\end{array}$ \\
\hline AD6 & TTGIAGNACIANAGG* & $16 \mathrm{X}$ & $\begin{array}{l}\text { Qiu et al., } 2010 \\
\text { (AD11) }\end{array}$ \\
\hline
\end{tabular}

*I-inosine

Table S3. Specific primers used for the TAIL-PCR based isolation of hop Ve1 sequences.

\begin{tabular}{|c|c|c|c|c|c|}
\hline & $1^{\circ}$ TAIL PCR & & $2^{\circ}$ TAIL PCR & & $3^{\circ}$ TAIL PCR \\
\hline Name & Oligonucleotide sequence $\left(5^{\prime} \rightarrow 3^{\prime}\right)$ & Name & Oligonucleotide sequence $\left(5^{\prime} \rightarrow 3^{\prime}\right)$ & Name & Oligonucleotide sequence $\left(5^{\prime} \rightarrow 3^{\prime}\right)$ \\
\hline VeT1F1 & TTTGGGAACTTGGCAATGGGAATCTTATC & VeT1F2 & GATGAGCCTGCAGGAACCATATTCTC & VeT1F3 & GCCTGCAGGAACCATATTCTCCTC \\
\hline VeT1R1 & GTTTTGAACCAGCATCCCCAAATCC & VeT1R2 & ATCCCCAAATCCGGGTTCTCAAGTTTC & VeT1R3 & GTGGAGATATCAAGAGTGACCAAGTTTGTC \\
\hline Ve1T2F1 & CACATGGAGCTCAAATTCAGATTG & Ve1T2F2 & AGTTTAGATGGCGAGGGACGT & Ve1T2F3 & GCTTGAGCAGTGAGTGGATCTCT \\
\hline Ve2T2F1 & TCACATGGAGTCAAAATTCAGATTG & $\mathrm{Ve} 2 \mathrm{~T} 2 \mathrm{~F} 2$ & TAACTTTGGATGGTGAGGGACG & Ve2T2F3 & TTGAGCAATGAGCGGATCTCT \\
\hline K1T2F1 & ATTCCCGATGCCTTTCCG & K1T2F2 & TTGGGAACAATGATATGAGTGGTG & K1T2F3 & TGGTGATTTTCCATGCTTGTTG \\
\hline K1T2R1 & AGCACTTGAAGATAGGGTGCATTA & K1T2R2 & TTACATATGGATTTTGGCACAATACC & K1T2R3 & GTCTGCGGGAATTGAGGAAGT \\
\hline К2Т2F1 & GCTCAATTCATGATGCCTTTCC & К2Т2F2 & CTTGGGAACAATCATATGAGTGGTAC & К2Т2F3 & GTGGTACTTTTCCTTGCTTGTTGA \\
\hline K2T2R1 & TCAAGCACTTGAAGGTAGGTTGC & K2T2R2 & CATATGGATTTTGG CATAGTTCCTATAA & К2T2R3 & ATGTCTGTGGGAATTGAGCAAGA \\
\hline Ve1T3F1 & САACTTCATGGGGAAATACCTTATTG & Ve1T3F2 & ССАТСТАAАТСТTТСССАСАAТСАТ & Ve1T3F3 & CTAACCAGCTCCAAGGGAAGATT \\
\hline $\mathrm{Ve} 1 \mathrm{~T} 4 \mathrm{~F} 1$ & TCACAGGCAGAATCCCATCAT & $\mathrm{Ve} 1 \mathrm{~T} 4 \mathrm{~F} 2$ & ACACCTGGAGTCCTTAGACCTCTC & Ve1T4F3 & AАTCCСATCAACСCTTGCAA \\
\hline Ve2PR1 & СТGСAATCTGAATTTTGACTCCAT & Ve2PR2 & TGTCCTTCAGTTGAAGCAACAAAG & Ve2PR3 & GTAAGGCATTGACCAGAGACGA \\
\hline
\end{tabular}

Primers are listed according to the TAIL-PCR procedure, consisting of three steps $\left(1^{\circ}-\right.$ primary, $2^{\circ}-$ secondary, $3^{\circ}-$ tertiary $)$ in which each was utilized.

Table S4. Amplification conditions used for TAIL-PCR (Liu et al., 1995).

\begin{tabular}{|c|c|c|}
\hline Level of TAIL-PCR & No. of cycles & Step in amplification \\
\hline \multirow[t]{7}{*}{$1^{\circ}$} & 1 & $1 \min 93^{\circ} \mathrm{C}, 1 \min 95^{\circ} \mathrm{C}$ \\
\hline & 5 & $1 \min 94^{\circ} \mathrm{C}, 1 \min 62^{\circ} \mathrm{C}, 2.5 \min 72^{\circ} \mathrm{C}$ \\
\hline & 1 & $1 \mathrm{~min} 94^{\circ} \mathrm{C}, 2 \mathrm{~min}$ cooling to $72^{\circ} \mathrm{C}, 2.5 \min 72^{\circ} \mathrm{C}$ \\
\hline & 15 & $30 \mathrm{~s} 94^{\circ} \mathrm{C}, 1 \min 68^{\circ} \mathrm{C}, 2.5 \min 72^{\circ} \mathrm{C}$ \\
\hline & & $30 \mathrm{~s} 94^{\circ} \mathrm{C}, 1 \min 68^{\circ} \mathrm{C}, 2.5 \min 72^{\circ} \mathrm{C}$ \\
\hline & & $30 \mathrm{~s} 94^{\circ} \mathrm{C}, 1 \min 44^{\circ} \mathrm{C}, 2.5 \min 72^{\circ} \mathrm{C}$ \\
\hline & 1 & $5 \min 72^{\circ} \mathrm{C}$ \\
\hline \multirow[t]{4}{*}{$2^{\circ}$} & 12 & $30 \mathrm{~s} 94^{\circ} \mathrm{C}, 1 \min 68^{\circ} \mathrm{C}, 2.5 \min 72^{\circ} \mathrm{C}$ \\
\hline & & $30 \mathrm{~s} 94^{\circ} \mathrm{C}, 1 \min 68^{\circ} \mathrm{C}, 2,5 \min 72^{\circ} \mathrm{C}$ \\
\hline & & $30 \mathrm{~s} 94^{\circ} \mathrm{C}, 1 \min 44^{\circ} \mathrm{C}, 2,5 \min 72^{\circ} \mathrm{C}$ \\
\hline & 1 & $5 \min 72^{\circ} \mathrm{C}$ \\
\hline \multirow[t]{2}{*}{$3^{\circ}$} & 20 & $1 \min 94^{\circ} \mathrm{C}, 1 \min 44^{\circ} \mathrm{C}, 2.5 \min 72^{\circ} \mathrm{C}$ \\
\hline & 1 & $5 \min 72^{\circ} \mathrm{C}$ \\
\hline
\end{tabular}




\section{REFERENCES}

Fei J, Chai Y, Wang J, Lin J, Sun X, Sun C, Tang K (2004) cDNA cloning and characterization of the Ve homologue gene StVe from Solanum torvum Swartz. DNA Seq 15: 88-95.

Fradin EF, Abd-El-Haliem A, Masini L, van den Berg GC, Joosten MH, Thomma BPHJ (2011) Interfamily transfer of tomato Ve1 mediates Verticillium resistance in Arabidopsis. Plant Physiol 156: 2255-2265.

Liu YG, Mitsukawa N, Oosumi T, Whittier RF (1995) Efficient isolation and mapping of Arabidopsis thaliana T-DNA insert junctions by thermal asymmetric interlaced PCR. Plant J 8: 457-463.

Liu S, Zhu Y, Xie C, Jue D, Hong Y, Chen M, Hubdar AK, Yang Q. (2012) Transgenic potato plants expressing StoVe1 exhibit enhanced resistance to Verticillium dahliae. Plant Mol. Biol. Rep 30: 1032-1039.

Maniatis T, Fritsch EF, Sambrook J (1982) Molecular cloning: a laboratory manual. Cold Spring Harbor Laboratory Cold Spring Harbor, NY.

Qiu, Z., Shi, P., Luo, H., Bai, Y., Yuan, T., Yang, P., Liu, S., Yao, B. (2010) A xylanase with broad pH and temperature adaptability from Streptomyces megasporus DSM 41476, and its potential application in brewing industry. Enzyme Microb Tech 46: 506-512.

Xu X, Pan S, Cheng S, Zhang B, Mu D, Ni P, Zhang G, Yang S, Li R, Wang J, et al. (2011) Genome sequence and analysis of the tuber crop potato. Nature 475: 189-195.

Zhang Z, Fradin E, de Jonge R, van Esse HP, Smit P, Liu CM, Thomma BPHJ (2013) Optimized agroinfiltration and virus-induced gene silencing to study Ve1-mediated Verticillium resistance in tobacco. Mol Plant Microbe Interact 26: 182-190. 



\section{Chapter 5}

\section{Transfer of tomato immune receptor Ve1 confers Ave1- dependent Verticillium resistance in tobacco and cotton}

Yin Song ${ }^{1}$, Linlin Liu' ${ }^{2}$, Yidong Wang ${ }^{1}$, Dirk-Jan Valkenburg ${ }^{1}$, Xianlong Zhang' ${ }^{2}$, Longfu Zhu $^{2}$ and Bart P.H.J. Thomma ${ }^{1}$

${ }^{1}$ Laboratory of Phytopathology, Wageningen University, Droevendaalsesteeg 1, 6708 PB Wageningen, the Netherlands

${ }^{2}$ National Key Laboratory of Crop Genetic Improvement, Huazhong Agricultural University, Wuhan, Hubei 430070, China

This chapter has been published as:

Song Y, Liu L, Wang Y, Valkenburg DJ, Zhang X, Zhu L, Thomma BPHJ (2017) Transfer of tomato immune receptor Ve1 confers Ave1-dependent Verticillium resistance in tobacco and cotton. Plant Biotechnol J DOI:10.1111/pbi.12804. 


\title{
Chapter 5
}

\begin{abstract}
Verticillium wilts caused by soil-borne fungal species of the Verticillium genus are economically important plant diseases that affect a wide range of host plants, and are notoriously difficult to combat. Perception of pathogen(-induced) ligands by plant immune receptors is a key component of plant innate immunity. In tomato, race-specific resistance to Verticillium wilt is governed by the cell surface-localized immune receptor Ve1 through recognition of the effector protein Ave1 that is secreted by race 1 strains of Verticillium spp. It was previously demonstrated that transgenic expression of tomato Ve1 in the model plant Arabidopsis thaliana leads to Verticillium wilt resistance. Here, we investigated whether tomato Ve1 can confer Verticillium resistance when expressed in the crop species tobacco (Nicotiana tabcum) and cotton (Gossypium hirsutum). We show that transgenic tobacco and cotton plants constitutively expressing tomato Ve1 exhibit enhanced resistance against Verticillium wilt in an Ave1-dependent manner. Thus, we demonstrate that the functionality of tomato Ve1 in Verticillum wilt resistance through recognition of the Verticillium effector Ave1 is retained after transfer to tobacco and cotton, implying that the Ve1-mediated immune signalling pathway is evolutionary conserved across these plant species. Moreover, our results suggest that transfer of tomato Ve1 across sexually incompatible plant species can be exploited in breeding programmes to engineer Verticillium wilt resistance.
\end{abstract}




\section{INTRODUCTION}

In order to activate immune responses to ward off invading microorganisms, plants deploy immune receptors that detect pathogen invasion through sensing pathogen(-induced) ligands (Dodds and Rathjen, 2010; Thomma et al., 2011; Cook et al., 2015). The recognition of such ligands results in the activation of defense responses, which are sometimes accompanied by a hypersensitive response (HR) in which plant tissue surrounding the site of attempted penetration is sacrificed to restrict further pathogen invasion. Based on structure and subcellular location, immune receptors fall into two major classes; cell surface localized receptors that comprise receptor kinases (RKs) and receptor-like proteins (RLPs) that monitor the extracellular space, and cytoplasm-localized nucleotide-binding domain leucine-rich repeat receptors (NLRs) that survey the intracellular environment (Rodriguez-Moreno et al., 2017).

Verticillium wilts are vascular wilt diseases caused by soil-borne fungal pathogens that belong to the Verticillium genus (Fradin and Thomma, 2006; Klimes et al., 2015). Although Verticillium wilt symptoms may vary considerably between plant hosts, the most frequently observed symptoms of Verticillium wilt include stunting, wilting, chlorosis, necrosis, vascular discoloration and early senescence (Fradin and Thomma, 2006). Within the Verticillium genus, V. dahliae is the most notorious pathogenic species that can infect hundreds of dicotyledonous hosts, including ecologically important plants and many high-value crops worldwide (Fradin and Thomma, 2006; Klosterman et al., 2009). V. albo-atrum, V. alfalfae, V. nonalfalfae and $V$. longisporum are also economically important vascular pathogens, albeit with narrower host ranges (Pegg and Brady, 2002; Agrios, 2005; Fradin and Thomma, 2006; Klosterman et al., 2009; Inderbitzin et al., 2011; Depotter et al., 2016). Verticillium wilt diseases are difficult to control due to the long viability of the resting structures, the wide host range of the pathogens, and the inability of fungicides to affect the pathogen once in the plant vascular system. Thus, the most sustainable manner to control Verticillium wilt diseases is the use of resistant cultivars (Fradin and Thomma, 2006; Klosterman et al., 2009).

In tomato (Solanum lycopersicum), a single dominant locus that confers Verticillium resistance has been identified as the Ve locus, which controls Verticillium isolates that are assigned to race 1 whereas race 2 strains of 


\section{Chapter 5}

Verticillium escape recognition (Schaible et al., 1951; Pegg, 1974). The Ve locus contains two closely linked and inversely oriented genes, $V e 1$ and $V e 2$, both of which encode extracellular leucine-rich repeat (eLRR) RLPs (Kawchuk et al., 2001; Wang et al., 2010). Of these, only Ve1 was found to confer resistance against race 1 isolates of Verticillium in tomato (Fradin et al., 2009). Interestingly, interfamily transfer of Ve1 from tomato to Arabidopsis thaliana leads to race-specific Verticillium resistance in the latter species (Fradin et al., 2011, 2014; Zhang et al., 2014), implying that the underlying immune signalling cascade is evolutionary conserved (Fradin et al., 2011; Thomma et al., 2011). Moreover, homologs of tomato Ve1 that have the potential to recognize race 1 strains of $V$. dahliae have been characterized in other plant species, suggesting an ancient origin of the tomato immune receptor Ve1 (Song et al., 2017a).

Through comparative population genomics of race 1 and race 2 strains of $V$. dahliae, the effector protein that activates Ve1-mediated immunity was identified

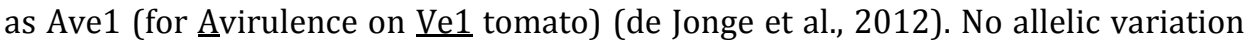
was found among the identified Ave1 alleles from $V$. dahliae as well as from $V$. alfalfae and V. nonalfalfae (de Jonge et al., 2012; Song et al., 2017b). Interestingly, homologues of Ave1 were found in the bacterial plant pathogen Xanthomonas axonopodis pv. citri (XacPNP) and in the plant-pathogenic fungi Colletotrichum higginsianum (ChAve1), Cercospora beticola (CbAve1), Fusarium oxysporum f. sp. lycopersici (FoAve1), as well as in plants (de Jonge et al., 2012). A few of these homologues are differentially recognized by tomato Ve1 in Nicotiana tabacum (de Jonge et al., 2012; Song et al., 2017b). Although the intrinsic function of Ave1 remains unknown, it is clear that Ave1 contributes to fungal virulence on susceptible plant genotypes (de Jonge et al., 2012).

Plant immune receptors are pivotal elements of the plant immune system that act as sentinels against pathogens. Engineering plants via transfer of immune components, such as plant immune receptors, has the potential to improve disease resistance in crops (Rodriguez-Moreno et al., 2017). Previous reports showed that the transfer of individual cell surface immune receptors into crops confers enhanced disease resistance against diverse pathogens, including bacteria, fungi and oomycetes. For example, transfer of the Arabidopsis cell surface immune receptor EFR results in responsiveness to bacterial elongation factor $\mathrm{Tu}$ and 
bacterial resistance in tomato (Lacombe et al., 2010), rice ( $\mathrm{Lu}$ et al., 2015; Schwessinger et al., 2015) and wheat (Schoonbeek et al., 2015). Similarly, introduction of the rice Xa21 confers bacterial resistance in sweet orange (Mendes et al., 2010), tomato (Afroz et al., 2011) and banana (Tripathi et al., 2014). Heterologous expression of the Nicotiana benthamiana FLS2 in citrus leads to increased disease resistance to citrus canker (Hao et al., 2016). Moreover, the Arabidopsis DORN1/LecRK-I.9 enhances resistance to Phytophthora infestans in potato (Bouwmeester et al., 2014). Finally, ectopic expression of the Arabidopsis RLP23 in potato plants enhances immunity to the oomycete and fungal plant pathogens P. infestans and Sclerotinia sclerotiorum (Albert et al., 2015). In this study, we investigated whether the immune receptor gene $V e 1$ can confer Verticillium wilt resistance when transferred from tomato to the closely related crop species tobacco ( $N$. tabacum cv. Samsun) and the distantly related crop species cotton (Gossypium hirsutum).

\section{RESULTS}

\section{Generation of Ve1-expressing Nicotiana tabacum plants}

Previously, it was shown that co-expression of Ve1 and Ave1 by agroinfiltration induces an HR in N. tabacum (Zhang et al., 2013a), suggesting that required signalling components acting downstream of tomato Ve1 are functionally conserved in tobacco. To further test whether tomato Ve1 can confer resistance to race 1 Verticillium spp., transgenic tobacco lines expressing tomato Ve1 were generated. The binary plasmid pSol2095_Ve1 encoding C-terminally eGFP-tagged Ve1 (Zhang et al., 2013a; Figure 1A) was transferred to N. tabacum cv. Samsun via Agrobacterium-mediated transformation. Primary transformants were selected in tissue culture by their ability to regenerate in the presence of kanamycin, and eight independent T0 transformation events expressing Ve1 were obtained (Figure S1A).

Intriguingly, 45 out of 56 progeny (T1 plants) derived from the eight T0 transformation events were significantly smaller in size when compared to the parental line, while 11 plants displayed a normal stature (Figure S1B and C). We assumed these 45 are Ve1-transgenic plants while the 11 correspond to segregating wild-type plants. To assay whether Ve1 protein accumulated in these plants, we isolated proteins from a line (Ve1 \#0) that exhibited normal growth and three lines 


\section{Chapter 5}

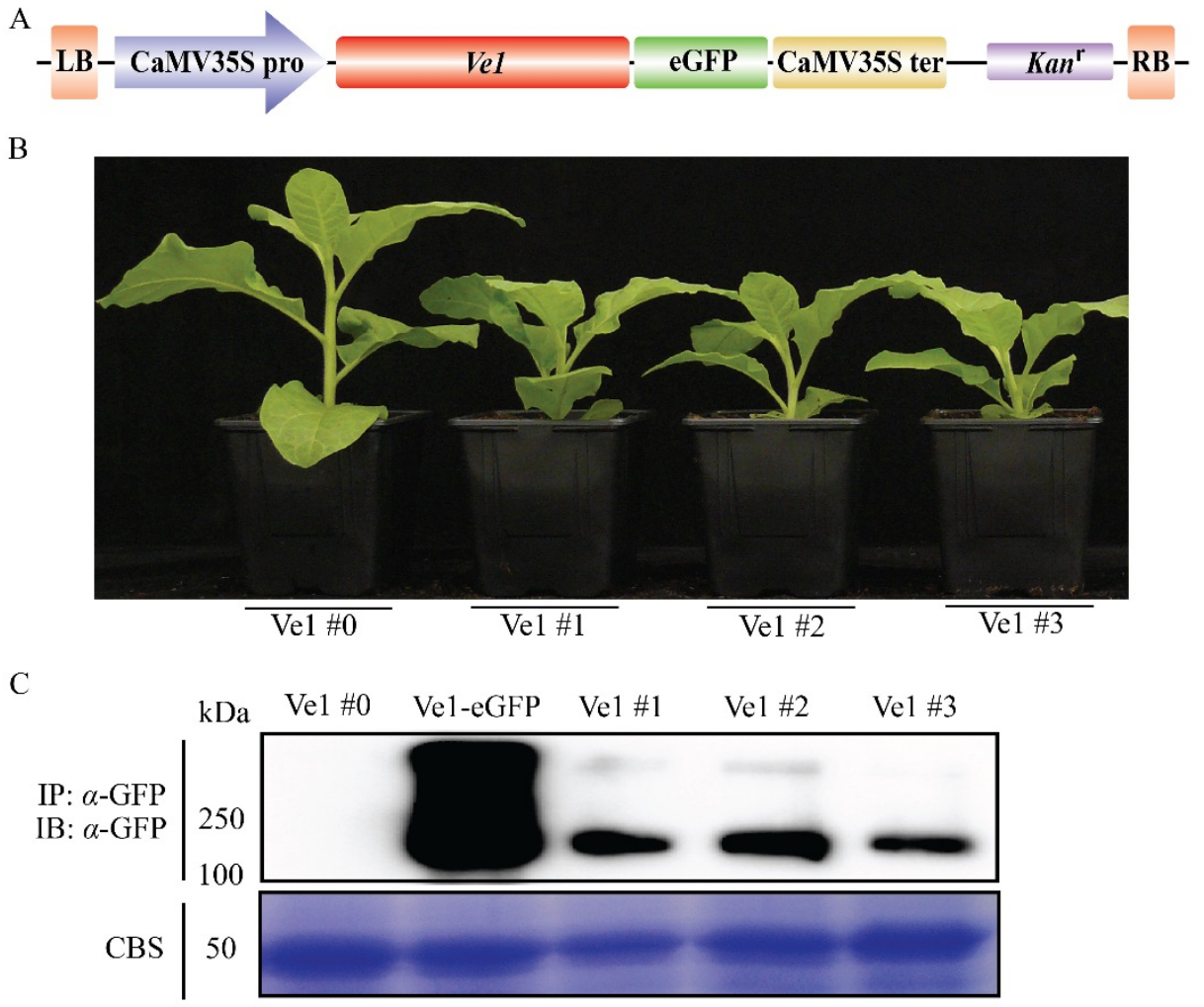

Figure 1. Generation and characterisation of Ve1-transgenic Nicotiana tabacum lines. (A) Schematic representation of the T-DNA region of the binary vector pSol2095_Ve1 used for tobacco transformation. CaMV35Spro: CaMV35S promoter, eGFP: enhanced green fluorescent protein, CaMV35Ster: CaMV35S terminator; Kanr: kanamycin resistance gene, LB and RB: left and right T-DNA borders, respectively. (B) Typical appearance of 6-week-old plants of four independent tobacco lines (Ve1 \#0, \#1, \#2 and \#3). (C) Accumulation of eGFP-tagged Ve1 protein ( 144 kDa) in leaves of the four independent $V e 1$ transgenic tobacco lines and wild-type tobacco cv. Samsun transiently expressing the eGFP-tagged Ve1 fusion protein (Ve1-eGFP). Total protein extracts of transformed leaf tissue were subjected to immunoprecipitation (IP) using $\alpha$-GFP affinity beads. Proteins were subjected to sodium dodecyl sulfate-polyacrylamide electrophoresis (SDS/PAGE) and immunoblotted (IB) using $\alpha$-GFP antibody. Coomassie blue staining (CBS) of the blot containing total protein extracts is shown as a loading control based on the 50-kDa RuBisCo (ribulose-1,5-bisphosphate carboxylase/oxygenase) band.

(Ve1 \#1, \#2 and \#3) that showed a dwarfed phenotype (Figure 1B), and performed immunoblotting analysis using anti-GFP-HRP antibody. This analysis showed that eGFP-tagged Ve1 protein was clearly detected in the three dwarfed lines, but not in the line that displays a normal stature (Figure 1C), suggesting that dwarfing of these tobacco lines is due to Ve1 expression. Nevertheless, the progeny of these 
three dwarfed lines was used for further assays to assess the contribution of Ve1 expression to Verticillium wilt resistance.

\section{Generation of isogenic Verticillium genotypes lacking or expressing Ave1}

To identify Verticillium strains that can be used for inoculation assays on tobacco, six strains, comprising V. dahliae strains JR2 and St14.01, V. nonalfalfae strains Vna5431, CBS385.91 and Vna1, and V. alfalfae strain Va2 (Table S1) were inoculated onto wild-type tobacco cv. Samsun plants, and Verticillium wilt symptoms were scored up to 14 days post inoculation (dpi). As anticipated, the various Verticillium strains caused different degrees of Verticillium wilt symptoms on these plants (Figure S2A). Among these six Verticillium genotypes, V. alfalfae strain Va2 and $V$. nonalfalfae strain Vna5431 caused the most severe disease symptoms (Figure S2A and B) and were selected for Verticillium wilt disease assays on tobacco. As $V$. nonalfalfae Vna5431 carries the Ave1 gene, it belongs to race 1, while V. alfalfae Va2 belongs to race 2 as it lacks Ave1 (Figure S2C).

In order to thoroughly investigate Ve1-mediated Verticillium wilt resistance in tobacco, isogenic Verticillium genotypes lacking or expressing Ave1 are required. To this end, we deleted Ave1 from the genome of $V$. nonalfalfae Vna5431, and simultaneously introduced Ave1 into V. alfalfae Va2 genome. Subsequently, the wild-type $V$. nonalfalfae strain Vna5431 and two independent Ave1 deletion mutants were used to inoculate Ve1 tomato plants and tomato plants that lack Ve1 (Figure S3A). As expected, targeted deletion of Ave1 resulted in gain of virulence on Ve1 tomato plants (Figure S3B and C). Moreover, these Ave1 deletion mutants displayed reduced virulence on tomato plants lacking Ve1 when compared to the corresponding wild-type $V$. nonalfalfae strain Vna5431 (Figure S3B and D). These results show that, also for $V$. nonalfalfae strain Vna5431 Ave1 acts as a virulence factor on tomato, and confirm that deletion of Ave1 leads to escape of Ve1-mediated resistance.

Simultaneously, the wild-type V. alfalfae strain Va2 and two independent Ave1 expression strains were inoculated onto tomato plants that express or lack Ve1 (Figure S4A). However, the wild-type V. alfalfae strain Va2, as well as the two Ave1expressing $V$. alfalfae strains, failed to cause visible disease symptoms on tomato plants (Figure S4B, D and E), suggesting that the $V$. alfalfae strain Va2 does not have 


\section{Chapter 5}

the capacity to infect tomato. Subsequently, we inoculated these strains on $N$. glutinosa, which is resistant to race 1 strains of Verticillium due to the occurrence of an endogenous Ve1 allele (Zhang et al., 2013a; Song et al., 2017a). As expected, V. alfalfae strain Va2 was able to infect $N$. glutinosa plants, while the Ave1 expression strains failed to cause infection (Figure S4C and F). These results reveal that ectopic expression of Ave1 in $V$. alfalfae strain Va2 can activate Ve1-mediated resistance against Verticillium wilt.

\section{Ave1 acts as a virulence factor on tobacco}

It was previously determined that Ave 1 acts as a virulence factor of $V$. dahliae on tomato and A. thaliana (de Jonge et al., 2012). To investigate the contribution of Ave1 to Verticillium virulence on tobacco, isogenic Ave1 mutants and the corresponding wild-type Verticillium strain were inoculated onto tobacco cv. Samsun plants. Interestingly, Ave1 deletion strains of V. nonalfalfae Vna5431 displayed significantly reduced virulence on tobacco plants when compared with the corresponding wild-type $V$. nonalfalfae strain Vna5431 at 21 dpi (Figure S5A), as inoculation with the Ave1 deletion strains resulted in reduced stunting (Figure S5B) and compromised fungal colonization (Figure S5C). Conversely, the two Ave1 expression strains in $V$. alfalfae Va2 showed clearly increased aggressiveness on tobacco plants when compared with the corresponding wild-type strain (Figure S5D-F). These experiments show that Ave1 acts as a virulence factor on tobacco plants.

\section{Tomato Ve1 confers Ave1-dependent Verticillium wilt resistance in tobacco}

To test if constitutive Ve1 expression in tobacco confers resistance against Verticillium wilt in an Ave1-dependent manner, three independent Ve1-transgenic lines (Ve1 \#1, \#2 and \#3) as well as non-transgenic control plants were challenged with either the wild-type race $1 \mathrm{~V}$. nonalfalfae strain Vna5431, or an Ave1 deletion mutant ( $V$. nonalfalfae Vna5431 $A$ Ave1) and inspected for Verticillium wilt symptoms up to $21 \mathrm{dpi}$. Interestingly, Ve1-transgenic tobacco plants were clearly more resistant to the race $1 \mathrm{~V}$. nonalfalfae strain Vna5431, as significantly fewer Verticillium wilt symptoms developed when compared with non-transgenic controls (Figure 2A and B). Importantly, despite the fact that the Ave1 deletion 

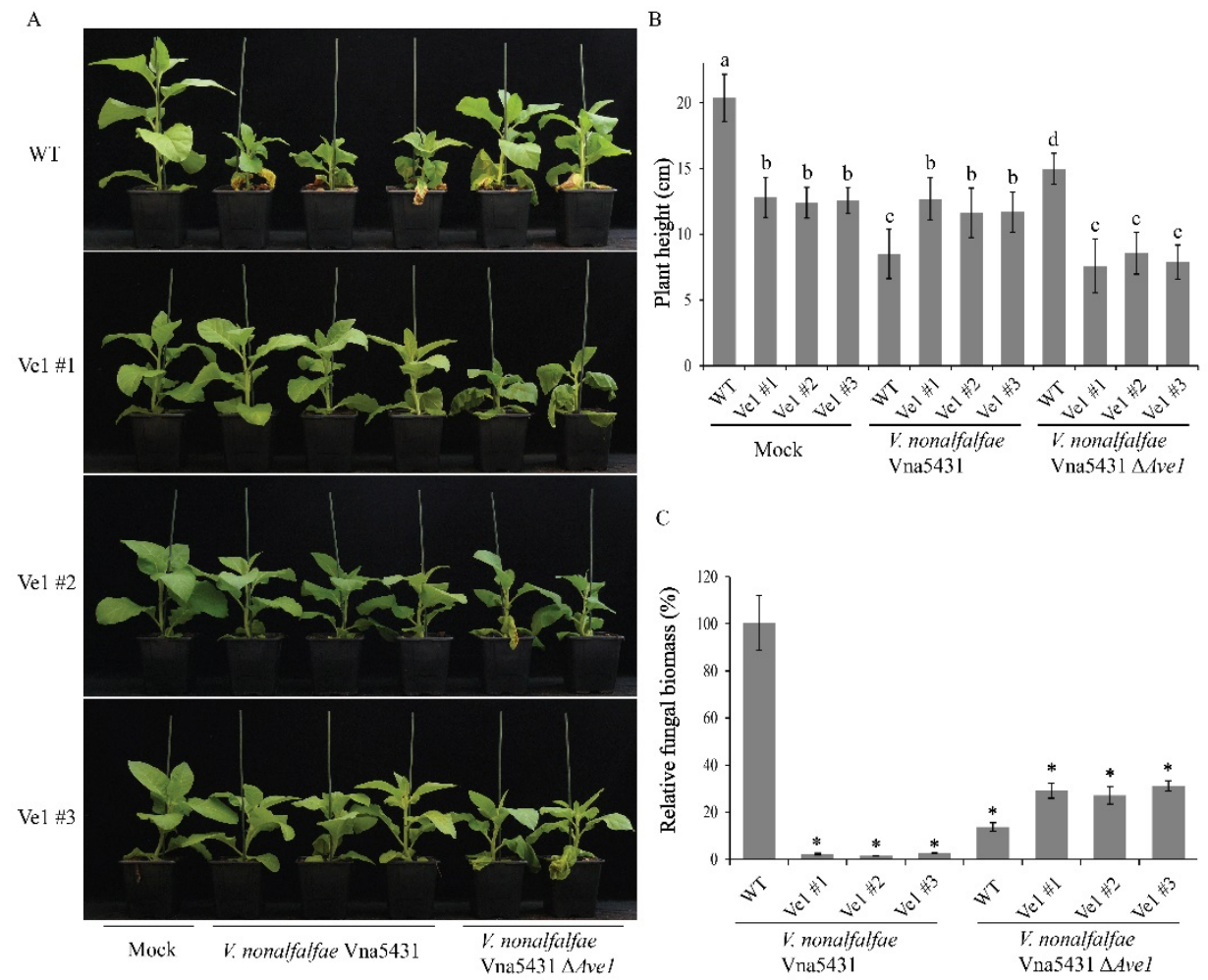

Figure 2. Tobacco plants expressing Ve1 show Ave1-triggered resistance against Verticillium nonalfalfae. (A) Typical appearance of wild-type tobacco cultivar Samsun plants (WT) and three independent Ve1 transgenic tobacco plants (\#1, \#2 and \#3) that were engineered to express tomato Ve1 upon mock-inoculation, inoculation with Ave1-carrying V. nonalfalfae Vna5431, or an Ave1 deletion strain of $V$. nonalfalfae Vna5431 ( $\triangle A v e 1$ ) at 21 days post inoculation (dpi). Inoculation experiments were performed with at least 16 plants for each fungal strain and independently repeated three times. (B) Quantification of Verticillium-induced plant stunting at 21 dpi. Bars represent averages with standard deviation. Different letter labels indicate statistically significant differences (Student's $t$-test; $P<0.05$ ). (C) Fungal biomass as determined with real-time PCR at 21 dpi. Bars represent Verticillium ITS levels relative to tobacco actin levels (for equilibration) with standard deviation in a sample of three pooled plants. The fungal biomass in tobacco cv. Samsun plants upon inoculation with the wild-type $V$. nonalfalfae strain Vna5431 is set to $100 \%$. Asterisks indicate statistically significant differences when compared with tobacco cv. Samsun plants upon inoculation with the wild-type $V$. nonalfalfae strain Vna5431 (Student's $t$-test; $P<0.05$ ). The data shown are representative of three independent experiments. 

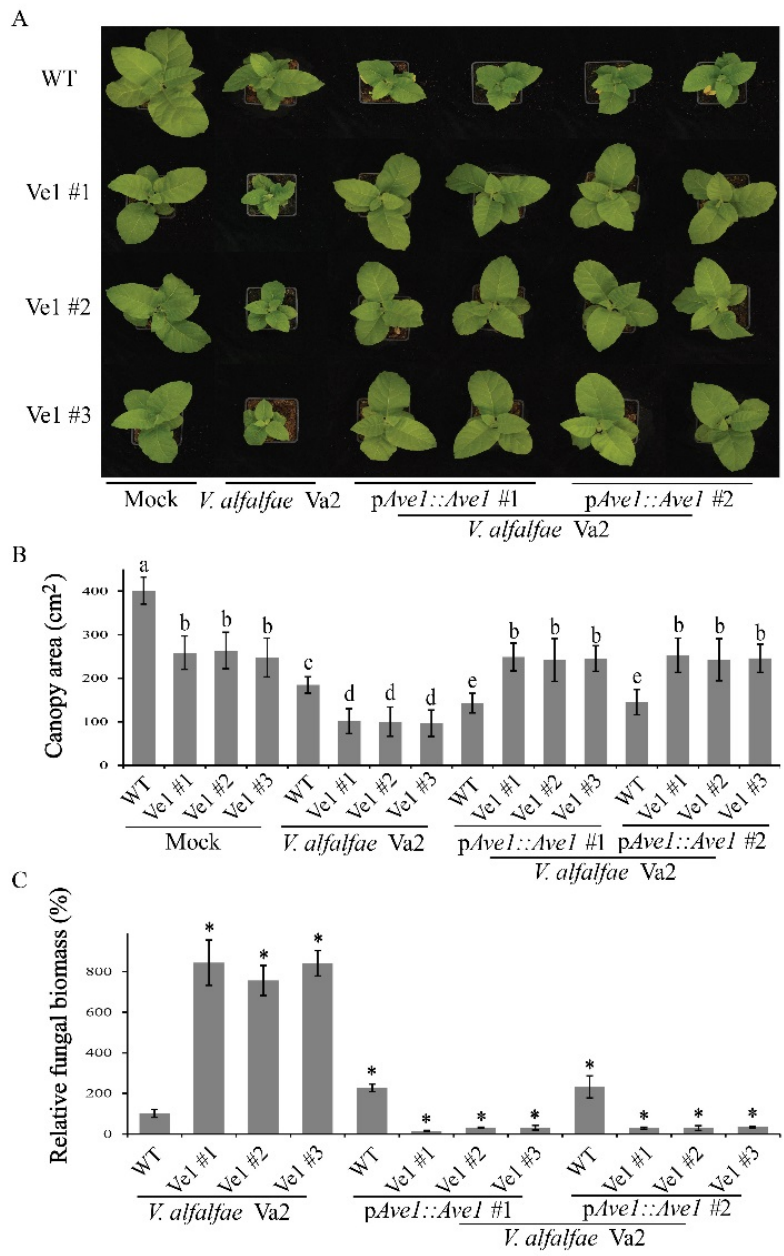

Figure 3. Tobacco plants expressing Ve1 exhibit Ave1-triggered resistance against Verticillium alfalfae. (A) Typical appearance of wild-type tobacco cultivar Samsun plants (WT) and three independent Ve1 transgenic tobacco plants (\#1, \#2 and \#3) that were engineered to express tomato Ve1 upon mock-inoculation, inoculation with V. alfalfae Va2, or two Ave1-experssing strains of V. alfalfae Va2 (pAve1::Ave1 \#1 and \#2) at 14 dpi. Inoculation experiments were performed with at least 16 plants for each fungal strain and independently repeated three times. (B) Quantification of the canopy area of tobacco plants at $14 \mathrm{dpi}$. Bars represent averages with standard deviation. Different letter labels indicate statistically significant differences (Student's $t$-test; $P<0.05$ ). (C) Fungal biomass as determined with real-time PCR at 14 dpi. Bars represent Verticillium ITS levels relative to tobacco actin levels (for equilibration) with standard deviation in a sample of three pooled plants. The fungal biomass in tobacco cv. Samsun plants upon inoculation with the wild-type V. alfalfae strain Va2 is set to $100 \%$. Asterisks indicate statistically significant differences when compared with tobacco cv. Samsun plants upon inoculation with the wild-type $V$. alfalfae strain Va2 (Student's $t$-test; $P<0.05$ ). The data shown are representative of three independent experiments. 
mutant of $V$. nonalfalfae Vna5431 displays compromised virulence on wild-type tobacco plants, Ve1-transgenic tobacco plants were clearly susceptible to this Ave1 deletion mutant (Figure 2A and B). The phenotypes correlated with the degree of fungal colonization as determined by real-time PCR (Figure 2C). Additionally, the three independent Ve1-transgenic tobacco lines and non-transgenic controls were inoculated with either the wild-type $V$. alfalfae strain Va2, or the two independent Ave1 expression strains, and monitored for the development of Verticillium wilt symptoms at $14 \mathrm{dpi}$. Intriguingly, upon inoculation with the Ave1 expression strains, no symptoms of Verticillium wilt were observed on the Ve1-expressing tobacco plants, whereas the non-transgenic controls displayed clear symptoms of Verticillium wilt (Figure 3). Importantly, all Ve1-transgenic lines were susceptible to the wild-type $V$. alfalfae strain Va2. Collectively, these data show that tobacco plants expressing Ve1 display enhanced Verticillium wilt resistance in an Ave1dependent manner.

\section{Generation of isogenic V. dahliae strains lacking Ave1}

Verticillium wilt of cotton is mostly caused by V. dahliae, and thus far effective tools to control Verticillium wilt in cotton are lacking (Cai et al., 2009). In a previous attempt to investigate whether tomato $V e 1$ can confer resistance against Verticillium wilt in cotton, transgenic cotton (G. hirsutum cv. YZ-1) lines that express tomato $V e 1$ were generated, but no increased Verticillium wilt resistance was observed (Liu et al., 2014). However, it was realized later on that the $V$. dahliae strains used in this study did not contain Ave1 (Liu et al., 2014).

To re-address the potential value of $V e 1$ to engineer Verticillium wilt resistance in cotton, we pursued Ave1-carrying $V$. dahliae that can cause clear Verticillium wilt symptoms on $G$. hirsutum cv. YZ-1 plants. To this end, we tested $V$. dahiae strains JR2, V4, V991 and V117 (Table S1) on cotton cultivar YZ-1, and the development of Verticillium wilt symptoms was monitored at $21 \mathrm{dpi}$. As expected, differential degrees of Verticillum wilt symptoms were observed on these cotton plants (Figure S6A). Whereas $V$. dahliae strain JR2 that carries Ave1 only induced mild symptoms on cotton, $V$. dahliae strain V4 that similarly carries Ave1, and $V$. dahliae strains V991 and V117 that both lack Ave1 induced considerably stronger 
Verticillium wilt symptoms (Figure S6). Thus, race $1 \mathrm{~V}$. dahliae strain V4 was selected for further assays on cotton.

In order to obtain an isogenic line that lacks Ave1, targeted replacement of Ave1 in $V$. dahliae strain V4 through homologous recombination was pursed (Figure S7A). To test whether the Ave1 deletion strains of $V$. dahliae V4 indeed overcome recognition by Ve1, two independent Ave1 deletion strains were inoculated onto tomato plants that express or lack Ve1 (Figure S7A). As expected, Ve1 tomato plants that were inoculated with two independent Ave1 deletion strains of V. dahliae V4 showed a similar disease phenotype as $V e 1$ tomato plants inoculated with the $V$. dahliae JR2 $\Delta$ Ave1 strain (Figure S7B and C; de Jonge et al., 2012), whereas Ve1 tomato plants inoculated with wild-type $V$. dahliae strains V4 and JR2 resembled mock-inoculated Ve1 tomato plants (Figure S7B and C). Moreover, the Ave1 deletion strains of $V$. dahliae strain V4 displayed significantly reduced virulence on susceptible tomato plants when compared with the corresponding wild-type race 1 V. dahliae strain V4 (Figure S7B and D). These results are in line with previous results show that Ave1 acts as a virulence factor on tomato, and confirm that deletion of Ave1 leads to escape of Ve1-mediated Verticillium wilt resistance (de Jonge et al., 2012).

\section{Ave1 acts as a virulence factor on cotton}

To investigate whether Ave1 acts as a virulence factor on cotton, two independent Ave1 deletion strains and the corresponding wild-type strain V4 were used to inoculate cotton cv. YZ-1 plants. Interestingly, the Ave1 deletion strains of $V$. dahliae V4 displayed clearly reduced virulence on wild-type cotton plants when compared with the corresponding wild-type strain up to 28 dpi (Figure S8A), as inoculation with Ave1 deletion mutants resulted in significantly reduced stunting (Figure S8B) and compromised fungal colonization (Figure S8C). This assay demonstrates that Ave1 acts as a virulence factor also on cotton.

\section{Cotton plants expressing Ve1 exhibit enhanced Verticillium wilt resistance in an Ave1-dependent manner}

To investigate if cotton plants constitutively expressing tomato Ve1 display enhanced resistance against race $1 \mathrm{~V}$. dahliae, two Ve1-trangenic lines (Ve1-4 and 

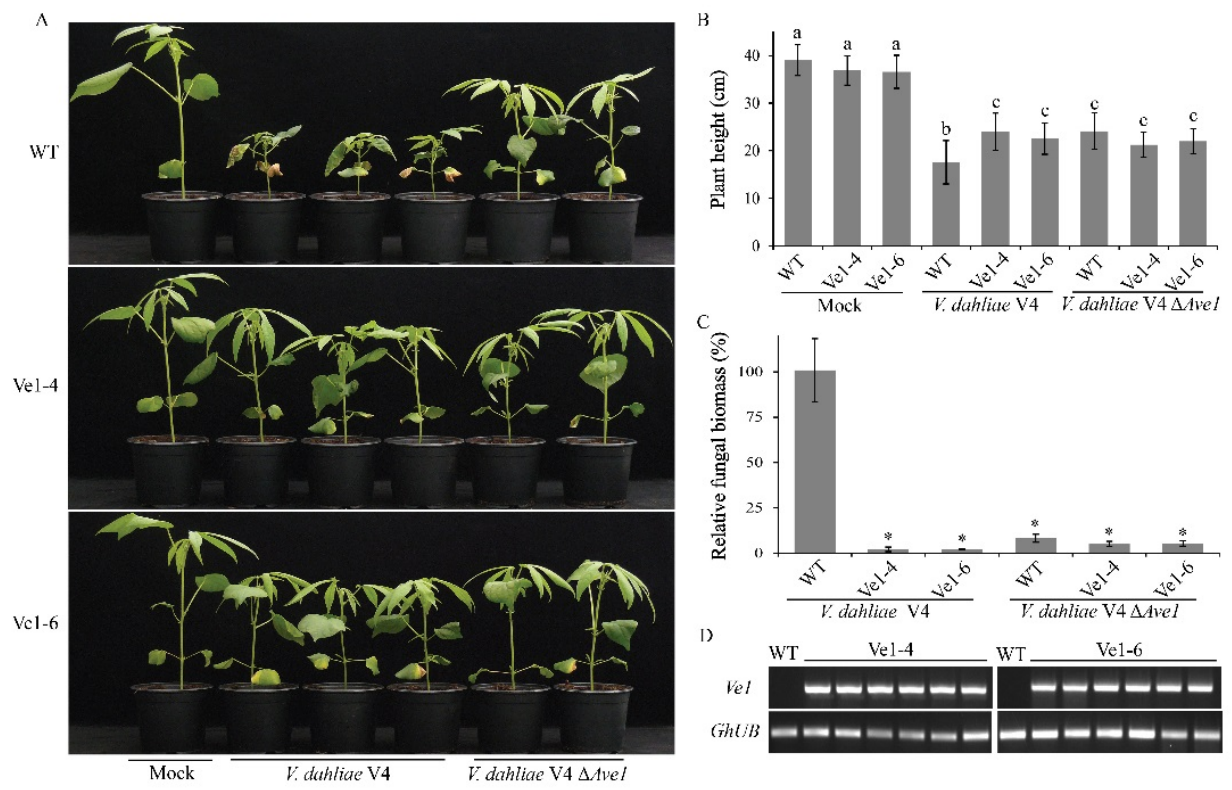

Figure 4. Cotton plants expressing Ve1 display Ave1-triggerd resistance against Verticillium dahliae. (A) Typical appearance of wild-type cotton cultivar YZ-1 plants (WT) and two independent Ve1 transgenic cotton plants (Ve1-4 and Ve1-6) upon mock inoculation, inoculation with Ave1-carrying $V$. dahliae V4, or an Ave1 deletion strain of $V$. dahliae V4 ( $\triangle$ Ave1) at 28 dpi. Inoculation experiments were performed with at least 20 plants for each fungal strain and independently repeated three times. (B) Quantification of Verticillium-induced plant stunting at 28 dpi. Bars represent averages with standard deviation. Different letter labels indicate statistically significant differences (Student's $t$-test; $P<0.05$ ). (C) Fungal biomass as determined with real-time PCR at 28 dpi. Bars represent Verticillium ITS levels relative to cotton ubiquitin levels (for equilibration) with standard deviation in a sample of three pooled plants. The fungal biomass in cotton cv. YZ-1 plants upon inoculation with the wild-type V. dahliae strain V4 is set to $100 \%$. Asterisks indicate statistically significant differences when compared with cotton cv. YZ-1 plants upon inoculation with the wild-type $V$. dahliae strain V4 (Student's $t$-test; $P<0.05$ ). (D) Expression of tomato Ve1 in individual transgenic cotton plants and non-transgenic controls of cotton cv. YZ-1 (WT) as detected with reverse transcription-PCR (RT-PCR). As an endogenous control, a fragment of the cotton ubiquitin gene (GhUB) was amplified. The data shown are representative of three independent experiments.

Ve1-6) as well as non-transgenic control plants were inoculated with either the race $1 \mathrm{~V}$. dahliae strain $\mathrm{V} 4$ or an Ave1 deletion mutant (V. dahliae V4LAve1), and monitored for Verticillium wilt symptoms up to $28 \mathrm{dpi}$. As expected, clear Verticillium wilt symptoms were observed on non-transgenic plants upon inoculation with $V$. dahliae strain V4 and with the corresponding Ave1 deletion mutant (Figure 4A-C), despite the observation that Ave1 deletion compromises 


\section{Chapter 5}

virulence on cotton. Interestingly, Ve1-expressing cotton plants exhibited significantly enhanced resistance against $V$. dahliae strain V4, as less Verticillium wilt symptoms were observed when compared with non-transgenic controls (Figure 4). When the two Ve1-transgenic lines and non-transgenic controls were challenged with $V$. dahliae strain V991 that does not carry Ave1, Ve1-expressing cotton lines were as susceptible as non-transgenic controls (Figure 5), confirming that the enhanced Verticillium wilt resistance upon Ve1 expression is Ave1dependent. Taken together, these data demonstrate that transfer of tomato immune receptor Ve1 into cotton confers Ave1-dependent Verticillium wilt resistance.

\section{DISCUSSION}

Major management strategies for Verticillium wilt diseases in crops include chemical and biological control, cultivation practices, and for the use of diseaseresistant cultivars (Fradin and Thomma, 2006; Klosterman et al., 2009). Although chemical control has been proven to be successful for many diseases and pests, no truly effective fungicides are commercially available to control Verticillium wilt diseases once plants have been infected (Fradin and Thomma, 2006; Klosterman et al., 2009). Biocontrol measures and cultivation practices for controlling Verticillium wilts are time-consuming and laborious, and control effectiveness largely depends on the field conditions. Therefore, breeding for disease-resistant cultivars has been considered as the most sustainable approach to control Verticillium wilt diseases in crops (Fradin and Thomma, 2006; Klosterman et al., 2009). Presently, genetic resistance against Verticillium spp. has been described in several plant species, including tomato, potato, hop, alfalfa, cotton, strawberry, sunflower and lettuce (Schaible et al., 1951; Putt, 1964; Barrow, 1970; Lynch et al., 1997; Simko et al., 2004; Bolek et al., 2005; Mert et al., 2005; Wang et al., 2008; Yang et al., 2008; Hayes et al., 2011; Jakse et al., 2013; Antanaviciute et al., 2015; Christopoulou et al., 2015). However, only tomato Ve1 has been cloned and characterized as a dominant gene responsible for race 1 Verticillium wilt resistance (Kawchuk et al., 2001; Fradin et al., 2009). We previously reported that homologues of tomato Ve1 occur widespread in phylogenetically distant plant species (Song et al., 2017a). However, despite being widespread, Ve1 homologues occur in a scattered fashion throughout plant phylogeny. For instance, a functional Ve1 allele was identified in N. glutinosa, 


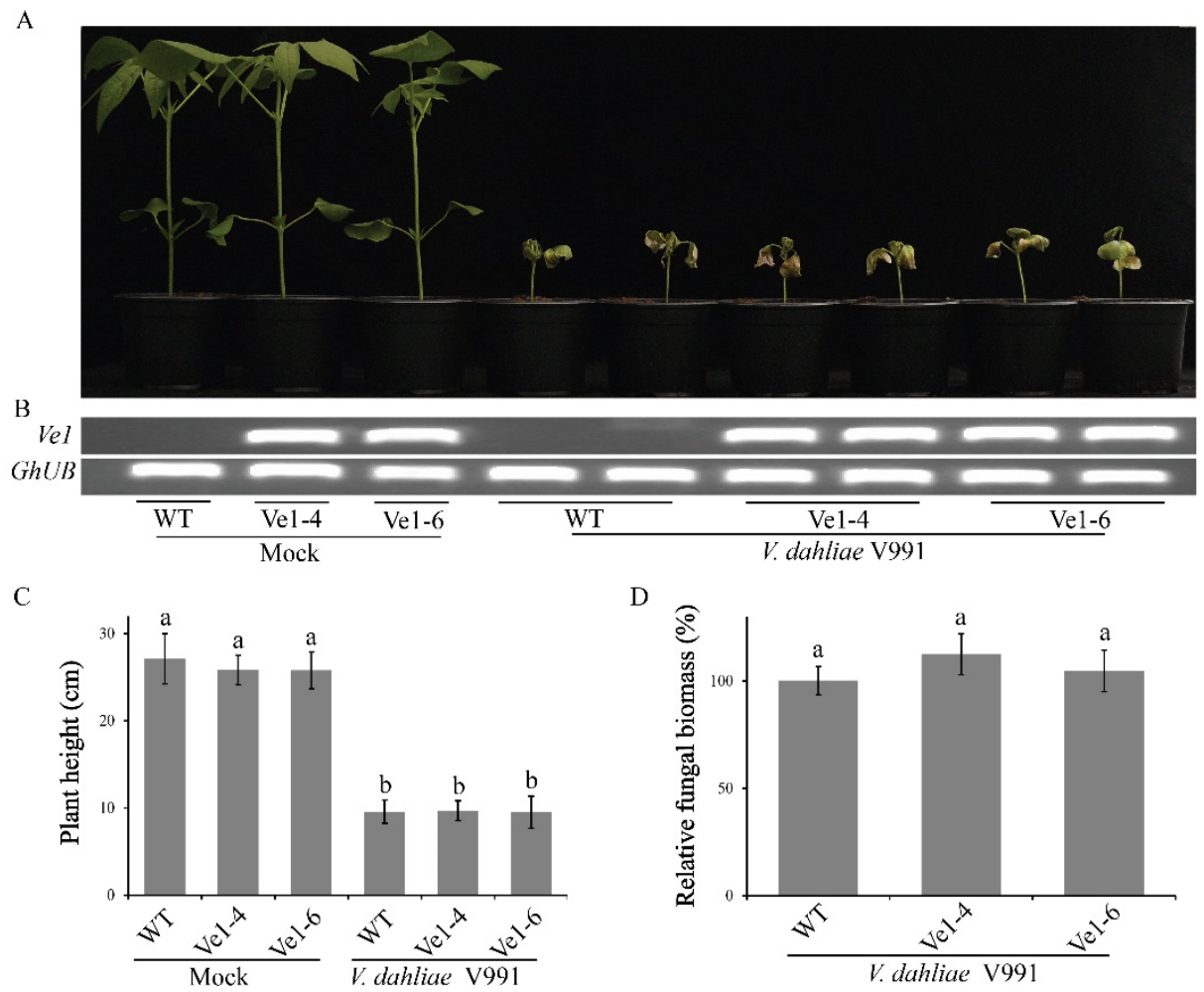

Figure 5. Ve1-transgenic and non-transgenic cotton plants are equally susceptible to Verticillium dahliae lacking Ave1. (A) Typical appearance of wild-type cotton cultivar YZ-1 plants (WT) and transgenic cotton plants expressing tomato Ve1 upon mock inoculation or inoculation with $V$. dahliae strain V991 at 28 dpi. Inoculation experiments were performed with at least 20 plants for $V$. dahliae strain V991 and independently repeated three times. (B) Expression of tomato Ve1 in individual cotton plants from wild-type controls and transgenic lines as detected with reverse transcription-PCR (RT-PCR). As an endogenous control, a fragment of the cotton ubiquitin gene (GhUB) was amplified. (C) Quantification of Verticillium-induced plant stunting at 28 dpi. Bars represent averages with standard deviation. Different letter labels indicate statistically significant differences (Student's $t$-test; $P<0.05$ ). (D) Fungal biomass as determined with real-time PCR at 28 dpi. Bars represent Verticillium ITS levels relative to cotton ubiquitin levels (for equilibration) with standard deviation in a sample of three pooled plants. The fungal biomass in cotton cv. YZ-1 plants upon inoculation with the V. dahliae strain V991 is set to $100 \%$. Same letter labels indicate no statistically significant differences (Student's $t$-test; $P>0.05$ ). The data shown are representative of three independent experiments.

but not in other species within the genus Nicotiana (Zhang et al., 2013a; Song et al., 2017a). This finding implies that, most likely, many plant species lost their functional Ve1 homologues, but underlying immune signalling cascade may have been retained. Indeed, we have previously shown that heterologous expression of 


\section{Chapter 5}

tomato $V e 1$ in the model plant $A$. thaliana that does not normally respond to Ave1, results in resistance against race 1 Verticillium spp. (Fradin et al., 2011; de Jonge et al., 2012; Zhang et al., 2013b). In this study, we investigated whether tomato Ve1 can confer Verticillium wilt resistance when expressed in the crop species tobacco and cotton. We show that transgenic tobacco and cotton plants constitutively expressing tomato Ve1 display enhanced resistance against Verticillium wilt in an Ave1-dependent manner. Thus, our results reveal that the functionality of tomato Ve1 in resistance against Verticillum wilt through recognition of the Verticillium effector Ave1 is retained after transfer to these plant species, and further support the view that the underlying immune signalling cascade mediated by Ve1 is retained in these plant species.

To date, several examples of transgenic expression of cell surface immune receptor genes resulting in enhanced disease resistance have been reported (Rodriguez-Moreno et al., 2017). Although transgenic expression of such receptors enhanced disease resistance, in some cases it also has adverse effects on plant fitness, such as growth retardation or leaf necrosis (Bouwmeester et al., 2011, 2014; Wang et al., 2016). In this study, we observed that expression of tomato Ve1 in tobacco caused stunted growth (Figure 1 and S1). A similar growth defect has previously been observed in N. benthamiana plants that constitutively express Ve1 (Fradin, 2011). In contrast, potato (Kawchuk et al., 2001), tomato (Fradin et al., 2009), A. thaliana (Fradin et al., 2011) and cotton (Figure 4 and 5) plants expressing Ve1 do not suffer from such growth defects. Based on these findings, we speculate that expression of tomato $V e 1$ in the genus Nicotiana may lead to a constitutive activation of downstream signalling cascade of tomato Ve1 that causes growth retardation. Alternatively, a ligand that is endogenous to these tobacco genotypes is recognized, leading to immune signalling activation.

Previously, we have shown that Ave1 acts as a virulence factor on tomato as well as on A. thaliana (de Jonge et al., 2012). In the present study we observed that targeted Ave1 deletion results in significantly compromised virulence of Verticillium spp. on tobacco (Figure S5) and cotton (Figure S8), demonstrating that Ave1 acts as a virulence factor also on these plants. Previously, the bacterial homolog XacPNP from $X$. axonopodis pv. citri was characterized as a virulence factor on citrus trees (Nembaware et al., 2004; Gottig et al., 2008). More recently, Ave1 homologs from 
the fungal tomato wilt pathogen F. oxysporum f. sp. lycopersici (FoAve1), and the fungal sugar beet leaf spot pathogen $C$. beticola (CbAve1) were characterized as virulence factors too. Although the intrinsic function of the fungal Ave1 homologs remains enigmatic, XacPNP is thought to manipulate the physiology of the host through plant natriuretic peptide activity that affects water homeostasis, stomatal opening, and photosynthesis to promote bacterial proliferation (Gottig et al., 2008; Garavaglia et al., 2010). It is tempting to speculate that the fungal homologs promote virulence through a similar activity, but this remains to be demonstrated. In any case, the finding that Ave1 promotes virulence on tomato, tobacco, cotton as well as A. thaliana suggests that the molecular target of the effector is widely conserved in the plant kingdom.

In summary, our data demonstrate that transfer of tomato Ve1 into the closely related crop species tobacco and the distantly related crop species leads to enhanced resistance against Verticillium wilt in an Ave1-dependent manner. Given that Ave1 homologues were found in a number of pathogenic microbes (Nembaware et al., 2004; de Jonge et al., 2012; Gan et al., 2013), and these homologs were differentially recognized by tomato Ve1 (de Jonge et al., 2012; Song et al., 2017b), our findings may further broaden biotechnological avenues to exploit tomato Ve1 for engineering disease resistance in an Ave1(homolog)-dependent manner, for instance through transfer or artificial evolution of tomato Ve1.

\section{MATERIALS AND METHODS}

\section{Plant materials and growth conditions}

Nicotiana tabacum cv. Samsun, N. glutinosa, cotton (Gossypium hirsutum cv. YZ-1), and tomato (Solanum lycopersicum cv. Moneymaker (ve1) or 35S::Ve1 tomato (Ve1); Fradin et al., 2009) plants were used in this study and grown in commercial potting soil (Horticoop, Bleiswijk, the Netherlands) under controlled greenhouse conditions (Unifarm, Wageningen, the Netherlands).

N. tabacum cv. Samsun seeds were surface-sterilized by $70 \%$ ethanol and $1 \%$ commercial bleach, and grown on Murashige-Skoog (MS) medium (4.4 g MS salt, 20 g sucrose and $8 \mathrm{~g}$ agar in $1 \mathrm{~L}$ ) or MS medium supplemented with antibiotics in a conditioned growth chamber at $21{ }^{\circ} \mathrm{C} / 19{ }^{\circ} \mathrm{C}$ during $16 \mathrm{~h} / 8 \mathrm{~h}$ light/dark photoperiods, respectively, and a relative humidity of $\sim 75 \%$. 


\section{Chapter 5}

\section{Generation of Ve1-transgenic plants}

Agrobacterium tumefaciens GV3101 (pMP90) carrying the binary vector pSol2095_Ve1 to encode C-terminally eGFP-tagged Ve1 (Figure 1A; Zhang et al., 2013a) was used for transformation of tobacco N. tabacum cv. Samsun. Transformation was performed by the leaf disk method as previously described (Wang et al., 2016). The generated plantlets were transferred to half-strength MS medium containing $200 \mathrm{mg} / \mathrm{L}$ kanamycin to allow root development. Upon root generation, plantlets were transferred into soil and grown in the greenhouse for seed production. Independent tobacco transformation lines were confirmed by PCR and reverse transcription-PCR (RT-PCR).

Two independent T0 generation cotton lines expressing tomato Ve1 gene driven by the cauliflower mosaic virus 35S promoter (Ve1-4 and Ve1-6; Liu et al., 2014) were self-pollinated to generate seeds. After two generations of selfing, T2 seeds were used for further experiments.

\section{Protein extraction, immunoprecipitation and immunoblotting}

To test whether eGFP-tagged Ve1 protein accumulated in transgenic tobacco lines, leaves of six-week-old transgenic tobacco lines were harvested and ground into a fine powder in liquid nitrogen. As a positive control, $A$. tumefaciens carrying the binary vector pSol2095_Ve1 was infiltrated into mature N. tabacum cv. Samsun leaves as described previously (Zhang et al., 2013a). Total proteins were extracted by using extraction buffer $\left(150 \mathrm{mM} \mathrm{NaCl}, 50 \mathrm{mM}\right.$ Tris-HCl pH 8.0, 1.0\% IGEPAL ${ }^{\circledR}$ CA-630 [NP-40] (Sigma-Aldrich Chemie BV, Zwijndrecht, the Netherlands) and one protease inhibitor cocktail tablet (Roche, Basel, Switzerland) per $50 \mathrm{~mL}$ extract buffer). Samples were centrifuged at $14,000 \mathrm{rpm}$ for $20 \mathrm{~min}$ at $4^{\circ} \mathrm{C}$, and then $2 \mathrm{~mL}$ of supernatant was incubated with $10 \mu \mathrm{L}$ (50\% slurry) of GFP-trap ${ }^{\circledR} \_$A beads (ChromoTek, Munich, Germany) at $4^{\circ} \mathrm{C}$ for $1 \mathrm{~h}$. After incubation, GFP-trap ${ }^{\circledR}$ AA beads with proteins were spun down by 1,000 rpm centrifugation and subsequently washed for six times in $1 \mathrm{~mL}$ extraction buffer. After each wash step the GFPtrap ${ }^{\circledR} \_$A beads were collected by 1,000 rpm centrifugation. Proteins were released from GFP-trap ${ }^{\circledR}$ A beads by boiling for 5 min, separated on a 10\% SDS-PAGE gel and wet-electroblotted onto PVDF membrane (Bio-Rad, Hercules, USA). Accumulation of eGFP-tagged Ve1 was detected by immunoblotting using anti-GFP- 
HRP antibody (Miltenyi Biotec, Bergisch Gladbach, Germany). SuperSignal ${ }^{\mathrm{TM}}$ West Femto Maximum Sensitivity Substrate (Thermo Scientfic, Waltham, USA) was used for signal development. Coomassie blue staining was used as loading control.

\section{Generation of Ave1 mutant strains}

Verticillium strains (Table S1) were grown on potato dextrose agar (PDA; Oxoid, Basingstoke, UK) at $22^{\circ} \mathrm{C}$. The Ave1 knockout construct pRF-HU2_Ave1 that was described previously (de Jonge et al., 2012) was used to generate Ave1 deletion mutants in V. nonalfalfae strain Vna 5431 and V. dahliae strain V4 (Table S1). The Ave1 complementation construct pFBT 005_pAve1::Ave1 that was described earlier (Song et al., 2017b) was used to generate Ave1 expression strains in V. alfalfae strain Va2 (Table S1).

Agrobacterium tumefaciens-mediated Verticillium transformation was performed as described previously (Santhanam, 2012), and Verticillium deletion transformants were selected on PDA (Oxoid, Basingstoke, UK) containing 200 $\mu \mathrm{g} / \mathrm{mL}$ cefotaxime and $50 \mu \mathrm{g} / \mathrm{mL}$ hygromycin (Duchefa, Haarlem, the Netherlands). Ave1 expression transformants were selected on PDA supplemented with 200 $\mu \mathrm{g} / \mathrm{mL}$ cefotaxime (Duchefa, Haarlem, the Netherlands) and $50 \mu \mathrm{g} / \mathrm{mL}$ nourseothricin sulphate (Sigma-Aldrich Chemie BV, Zwijndrecht, the Netherlands).

Putative Verticillium transformants were tested by PCR, and subsequent inoculation on Ve1 tomato plants (Ve1) and tomato cultivar Moneymaker plants (ve1) (Fradin et al., 2009), or N. glutionsa plants carrying a functional Ve1 homolog (Song et al., 2017a).

\section{Disease assays}

Verticillium conidiospores were collected from 7- to 10-day-old cultures on PDA plates and washed with tap water. Disease assays were performed on tomato, tobacco and cotton plants using the root-dipping inoculation method as previously described (Fradin et al., 2009). Briefly, 10-day-old Ve1 or ve1 tomato seedlings (for inoculation with Verticillium Ave1 deletion strains), or four-week-old tobacco ( $N$. tabacum cv. Samsun or N. glutinosa) plants or 10-day-old cotton seedlings were uprooted. Next, the roots were rinsed in water, dipped for $5 \mathrm{~min}$ in a suspension of $10^{6}$ conidiospores/mL water while the roots of mock plants (control) were dipped 


\section{Chapter 5}

in tap water without conidiospores, and subsequently transplanted to fresh commercial potting soil (Horticoop, Bleiswijk, the Netherlands). Disease symptoms were scored up to 14 days post inoculation (dpi) (tomato, N. glutinosa and $N$. tabacum cv. Samsun), or 21 dpi (N. tabacum cv. Samsun), or 28 dpi (cotton). To this end, plants were photographed, and Image J was used to determine the canopy area (for quantification of stunting) while the rectilinear scale was used to measure the plant height (for quantification of growth). For fungal biomass quantification in planta, stems of three inoculated plants were harvested at $14 \mathrm{dpi}$ (for $N$. tabacum cv. Samsun upon $V$. alfalfae inoculation), $21 \mathrm{dpi}$ (for $N$. tabacum cv. Samsun upon $V$. nonalfalfae inoculation), or $28 \mathrm{dpi}$ (cotton upon $V$. dahliae inoculation). The samples were ground into a fine powder in liquid nitrogen and genomic DNA was isolated. Real-time PCR was conducted by using the fungus-specific primers ITS-F and ITS-R (Table S2) with primers for tobacco actin (GenBank accession number: X69885; for Verticillium-infected tobacco) or cotton ubiquitin (GenBank accession number: DQ116441; for Verticillium-infected cotton) (Table S2) as an endogenous plant control, employing an ABI 7300 PCR system (Applied Biosystems, Foster City, CA, USA) with the qPCR Core kit for SYBR Green I (Eurogentec Nederland BV, Maastricht, the Netherlands).

\section{Gene expression analysis}

For the expression of $V e 1$ in transgenic tobacco plants, leaves of six-week-old tobacco plants were harvested and ground into a fine powder in liquid nitrogen. Tobacco total RNA isolation and cDNA synthesis were performed as previously described (Song et al., 2017a). RT-PCR was conducted by using the primers Ve1F(PCR) and Ve1-R(PCR) (Table S2), and N. tabacum actin gene (NtACT) (Table S2) was used as the endogenous control.

To check whether the Ve1 gene is expressed in individual T2 cotton plants, leaves of five-week-old cotton plants were collected separately, flash frozen in liquid nitrogen and stored at $-80^{\circ} \mathrm{C}$ for total RNA isolation. Cotton total RNA was isolated by using Spectrum ${ }^{\mathrm{TM}}$ Plant Total RNA Kit (Sigma-Aldrich Chemie BV, Zwijndrecht, the Netherlands) following the manufacturer's instructions. Firststrand cDNA synthesis was performed by using M-MLV reverse transcriptase system (Promega, Wisconsin, USA). RT-PCR was conducted with primers Ve1-F(RT) 
and Ve1-R(RT) (Table S2) in a total volume of $25 \mu$ l with 17.9 sterilized-water, $5 \mu \mathrm{l}$ 5x PCR buffer, $0.5 \mu \mathrm{l}$ dNTPs, $0.5 \mu \mathrm{l}$ of each primer, $0.1 \mu \mathrm{l}$ GoTag DNA polymerase (Promega, Wisconsin, USA) and $1.0 \mu$ of first-strand cDNA. Primers GhUb-F and GhUb-R (Table S2) were used to amplify the cotton ubiquitin gene as endogenous loading control. PCR amplification consisted of an initial denaturation step of $95^{\circ} \mathrm{C}$ for $5 \mathrm{~min}$, followed by denaturation at $95^{\circ} \mathrm{C}$ for $30 \mathrm{~s}$, annealing at $55^{\circ} \mathrm{C}$ for $30 \mathrm{~s}$, and extension at $72^{\circ} \mathrm{C}$ for $40 \mathrm{~s}$ with 35 cycles. The resulting PCR products were subjected to agarose gel electrophoresis.

\section{ACKNOWLEDGEMENTS}

Y.S. acknowledges a PhD fellowship from the China Scholarship Council (CSC). B.P.H.J.T. is supported by a Vici grant of the Research Council for Earth and Life sciences (ALW) of the Netherlands Organization for Scientific Research (NWO). L.L.L., X.L.Z. and L.F.Z. acknowledge grant 2015DFA30860 from the International Science \& Technology Cooperation Program of China. Bert Essenstam is acknowledged for excellent plant care at Unifarm. 


\section{REFERENCES}

Afroz A, Chaudhry Z, Rashid U, Ali GM, Nazir F, Iqbal J, Khan MR (2011) Enhanced resistance against bacterial wilt in transgenic tomato (Lycopersicon esculentum) lines expressing the Xa21 gene. Plant Cell Tissue Organ Cult 104: 227-237.

Agrios G (2005) Plant Pathology, $5^{\text {th }}$ edn. Elsevier Academic Press, New York.

Albert I, Böhm H, Albert M, Feiler CE, Imkampe J, Wallmeroth N, Brancato C, Raaymakers TM, Oome S, Zhang H, Krol E, Grefen C, Gust AA, Chai J, Hedrich R, Van den Ackerveken G, Nürnberger T (2015) An RLP23-SOBIR1-BAK1 complex mediates NLP-triggered immunity. Nat Plants 1: 15140.

Antanaviciute L, Šurbanovski N, Harrison N, McLeary K, Simpson D, Wilson F, Sargent D, Harrison $\mathbf{R}$ (2015) Mapping QTL associated with Verticillium dahliae resistance in the cultivated strawberry (Fragariax ananassa). Hortic Res 2: 15009.

Barrow JR (1970) Heterozygosity in inheritance of Verticillium wilt tolerance in cotton. Phytopathology 60: 301-303.

Bolek Y, El-Zik KM, Pepper AE, Bell AA, Magill CW, Thaxton PM, Reddy OUK (2005) Mapping of verticillium wilt resistance genes in cotton. Plant Sci 168: 1581-1590.

Bouwmeester K, De Sain M, Weide R, Gouget A, Klamer S, Canut H, Govers F (2011) The lectin receptor kinase LecRK-I. 9 is a novel Phytophthora resistance component and a potential host target for a RXLR effector. PLOS Pathog 7: e1001327.

Bouwmeester K, Han M, Blanco-Portales R, Song W, Weide R, Guo LY, van der Vossen EA, Govers F (2014) The Arabidopsis lectin receptor kinase LecRK-I. 9 enhances resistance to Phytophthora infestans in Solanaceous plants. Plant Biotechnol J 12: 10-16.

Cai Y, Xiaohong H, Mo J, Sun Q, Yang J, Liu J (2009) Molecular research and genetic engineering of resistance to Verticillium wilt in cotton: a review. Afr J Biotechnol 8: 87363-7372.

Christopoulou M, Wo SR-C, Kozik A, McHale LK, Truco M-J, Wroblewski T, Michelmore R (2015) Genome-wide architecture of disease resistance genes in lettuce. G3 5: 2655-2699.

Cook DE, Mesarich CH, Thomma BPHJ (2015) Understanding plant immunity as a surveillance system to detect invasion. Annu Rev Phytopathol 53: 541-563.

de Jonge R, van Esse HP, Maruthachalam K, Bolton MD, Santhanam P, Saber MK, Zhang Z, Usami T, Lievens B, Subbarao KV, Thomma BPHJ (2012) Tomato immune receptor Ve1 recognizes effector of multiple fungal pathogens uncovered by genome and RNA sequencing. Proc Natl Acad Sci USA 109: 5110-5115.

Depotter JR, Deketelaere S, Inderbitzin P, Tiedemann AV, Höfte M, Subbarao KV, Wood TA, Thomma BPHJ (2016) Verticillium longisporum, the invisible threat to oilseed rape and other brassicaceous plant hosts. Mol Plant Pathol 17: 1004-1016.

Dodds PN, Rathjen JP (2010) Plant immunity: towards an integrated view of plant-pathogen interactions. Nat Rev Genet 11: 539-548.

Fradin EF (2011) Functional analysis of the tomato Ve resistance locus against Verticillium wilt. Wageningen University \& Research PhD thesis.

Fradin EF, Abd-El-Haliem A, Masini L, van den Berg GC, Joosten MH, Thomma BPHJ (2011) Interfamily transfer of tomato Ve1 mediates Verticillium resistance in Arabidopsis. Plant Physiol 156: 2255-2265.

Fradin EF, Thomma BPHJ (2006) Physiology and molecular aspects of Verticillium wilt diseases caused by V. dahliae and V. albo-atrum. Mol Plant Pathol 7: 71-86. 
Fradin EF, Zhang Z, Ayala JCJ, Castroverde CD, Nazar RN, Robb J, Liu C-M, Thomma BPHJ (2009) Genetic dissection of Verticillium wilt resistance mediated by tomato Ve1. Plant Physiol 150: 320332.

Fradin EF, Zhang Z, Rovenich H, Song Y, Liebrand TW, Masini L, van den Berg GC, Joosten MH, Thomma BPHJ (2014) Functional analysis of the tomato immune receptor Ve1 through domain swaps with its non-functional homolog Ve2. PLOS ONE 9: e88208.

Gan P, Ikeda K, Irieda H, Narusaka M, O'Connell RJ, Narusaka Y, Takano Y, Kubo Y, Shirasu K (2013) Comparative genomic and transcriptomic analyses reveal the hemibiotrophic stage shift of Colletotrichum fungi. New Phytol 197: 1236-1249.

Garavaglia BS, Thomas L, Zimaro T, Gottig N, Daurelio LD, Ndimba B, Orellano EG, Ottado J, Gehring C (2010) A plant natriuretic peptide-like molecule of the pathogen Xanthomonas axonopodis pv. citri causes rapid changes in the proteome of its citrus host. BMC Plant Biol 10: 51.

Gottig N, Garavaglia BS, Daurelio LD, Valentine A, Gehring C, Orellano EG, Ottado J (2008) Xanthomonas axonopodis pv. citri uses a plant natriuretic peptide-like protein to modify host homeostasis. Proc Natl Acad Sci USA 105: 18631-18636.

Hao G, Pitino M, Duan Y, Stover E (2016) Reduced susceptibility to Xanthomonas citri in transgenic citrus expressing the FLS2 receptor from Nicotiana benthamiana. Mol Plant-Microbe Interact 29: 132-142.

Hayes RJ, McHale LK, Vallad GE, Truco MJ, Michelmore RW, Klosterman SJ, Maruthachalam K, Subbarao KV (2011) The inheritance of resistance to Verticillium wilt caused by race 1 isolates of Verticillium dahliae in the lettuce cultivar La Brillante. Theor Appl Genet 123: 509-517.

Inderbitzin P, Davis RM, Bostock RM, Subbarao KV (2011) The ascomycete Verticillium longisporum is a hybrid and a plant pathogen with an expanded host range. PLOS ONE 6: e18260.

Jakse J, Cerenak A, Radisek S, Satovic Z, Luthar Z, Javornik B (2013) Identification of quantitative trait loci for resistance to Verticillium wilt and yield parameters in hop (Humulus lupulus L.). Theor Appl Genet 126: 1431-1443.

Kawchuk LM, Hachey J, Lynch DR, Kulcsar F, van Rooijen G, Waterer DR, Robertson A, Kokko E, Byers R, Howard RJ (2001) Tomato Ve disease resistance genes encode cell surface-like receptors. Proc Natl Acad Sci 98: 6511-6515.

Klimes A, Dobinson KF, Klosterman SJ, Thomma BPHJ (2015) Genomics spurs rapid advances in our understanding of the basic biology of vascular wilt pathogens in the genus Verticillium. Annu Rev Phytopathol 53: 181-198.

Klosterman SJ, Atallah ZK, Vallad GE, Subbarao KV (2009) Diversity, pathogenicity, and management of Verticillium species. Annu Rev Phytopathol 47: 39-62.

Lacombe S, Rougon-Cardoso A, Sherwood E, Peeters N, Dahlbeck D, Van Esse HP, Smoker M, Rallapalli G, Thomma BPHJ, Staskawicz B (2010) Interfamily transfer of a plant patternrecognition receptor confers broad-spectrum bacterial resistance. Nat Biotechnol 28: 365-369.

Liu L, Zhang W, Zhou Y, Miao Y, Xu L, Liu M, Zhang K, Zhang X, Zhu L (2014) Resistance of cotton and tomato to Verticillium dahliae from cotton is independent on Ve1. Scia Sin Vitae 44: 803-814.

Lu F, Wang H, Wang S, Jiang W, Shan C, Li B, Yang J, Zhang S, Sun W (2015) Enhancement of innate immune system in monocot rice by transferring the dicotyledonous elongation factor Tu receptor EFR. J Integr Plant Biol 57: 641-652.

Lynch D, Kawchuk L, Hachey J, Bains P, Howard R (1997) Identification of a gene conferring high levels of resistance to Verticillium wilt in Solanum chacoense. Plant Dis 81: 1011-1014. 
Mendes B, Cardoso S, Boscariol-Camargo R, Cruz R, Mourão Filho F, Bergamin Filho A (2010) Reduction in susceptibility to Xanthomonas axonopodis pv. citri in transgenic Citrus sinensis expressing the rice Xa21 gene. Plant Pathol 59: 68-75.

Mert M, Kurt S, Gencer O, Akiscan Y, Boyaci K, Tok F (2005) Inheritance of resistance to Verticillium wilt (Verticillium dahliae) in cotton (Gossypium hirsutum L.). Plant Breed 124: 102-104.

Nembaware V, Seoighe C, Sayed M, Gehring C (2004) A plant natriuretic peptide-like gene in the bacterial pathogen Xanthomonas axonopodis may induce hyper-hydration in the plant host: a hypothesis of molecular mimicry. BMC Evol Biol 4: 10.

Pegg GF (1974) Verticillium diseases. Rev Plant Pathol 53: 157-182.

Pegg GF, Brady BL (2002) Verticillium wilts. Wallingford, Oxfordshire: CABI publishing.

Putt ED (1964) Breeding behaviour of resistance to leaf mottle or Verticillium in sunflowers. Crop Sci 4: 177-179.

Rodriguez-Moreno L, Song Y, Thomma BPHJ (2017) Transfer and engineering of immune receptors to improve recognition capacities in crops. Curr Opin Plant Biol 38: 42-49.

Santhanam P (2012) Random insertional mutagenesis in fungal genomes to identify virulence factors. In Plant Fungal Pathogens (Melvin D. Bolton and Bart P.H.J. Thomma eds), pp. 509-517. New York: Springer.

Schaible L, Cannon OS, Waddoups V (1951) Inheritance of resistance to Verticillium wilt in a tomato cross. Phytopathology 41: 986-990.

Schoonbeek Hj, Wang HH, Stefanato FL, Craze M, Bowden S, Wallington E, Zipfel C, Ridout CJ (2015) Arabidopsis EF-Tu receptor enhances bacterial disease resistance in transgenic wheat. New Phytol 206: 606-613.

Schwessinger B, Bahar O, Thomas N, Holton N, Nekrasov V, Ruan D, Canlas PE, Daudi A, Petzold CJ, Singan VR (2015) Transgenic expression of the dicotyledonous pattern recognition receptor EFR in rice leads to ligand-dependent activation of defense responses. PLOS Pathog 11: e1004809.

Simko I, Costanzo S, Haynes K, Christ B, Jones R (2004) Linkage disequilibrium mapping of a Verticillium dahliae resistance quantitative trait locus in tetraploid potato (Solanum tuberosum) through a candidate gene approach. Theor Appl Genet 108: 217-224.

Song Y, Zhang Z, Seidl MF, Majer A, Jakse J, Javornik B, Thomma BPHJ (2017a) Broad taxonomic characterization of Verticillium wilt resistance genes reveals an ancient origin of the tomato Ve1 immune receptor. Mol Plant Pathol 18: 195-209

Song Y, Zhang Z, Boshoven J, Rovenich H, Seidl M, Jakse J, Maruthachalam K, Liu C-M, Subbarao K, Javornik B, Thomma BPHJ (2017b) Tomato immune receptor Ve1 recognizes surface-exposed co-localized $\mathrm{N}$ - and C-termini of Verticillium dahliae effector Ave1. bioRxiv https://doi.org/10.1101/103473.

Thomma BPHJ, Nürnberger T, Joosten MH (2011) Of PAMPs and effectors: the blurred PTI-ETI dichotomy. Plant Cell 23: 4-15.

Tripathi JN, Lorenzen J, Bahar O, Ronald P, Tripathi L (2014) Transgenic expression of the rice Xa21 pattern-recognition receptor in banana (Musa sp.) confers resistance to Xanthomonas campestris pv. musacearum. Plant Biotechnol J 12: 663-673.

Wang G, Fiers M, Ellendorff U, Wang Z, de Wit PJ, Angenent GC, Thomma BPHJ (2010) The diverse roles of extracellular leucine-rich repeat-containing receptor-like proteins in plants. Crit Rev Plant Sci 29: 285-299.

Wang HM, Lin ZX, Zhang XL, Chen W, Guo XP, Nie YC, Li YH (2008) Mapping and quantitative trait loci analysis of verticillium wilt resistance genes in cotton. J Integr Plant Biol 50: 174-182. 
Wang Y, Nsibo DL, Juhar HM, Govers F, Bouwmeester K (2016) Ectopic expression of Arabidopsis Ltype lectin receptor kinase genes LecRK-I.9 and LecRK-IX.1 in Nicotiana benthamiana confers Phytophthora resistance. Plant Cell Rep 35: 845-855.

Yang C, Guo W, Li G, Gao F, Lin S, Zhang T (2008) QTLs mapping for Verticillium wilt resistance at seedling and maturity stages in Gossypium barbadense L. Plant Sci 174: 290-298.

Zhang Z, Fradin E, de Jonge R, van Esse HP, Smit P, Liu C-M, Thomma BPHJ (2013a) Optimized agroinfiltration and virus-induced gene silencing to study Ve1-mediated Verticillium resistance in tobacco. Mol Plant-Microbe Interact 26: 182-190.

Zhang Z, Esse HP, Damme M, Fradin EF, Liu C-M, Thomma BPHJ (2013b) Ve1-mediated resistance against Verticillium does not involve a hypersensitive response in Arabidopsis. Mol Plant Pathol 14: 719-727.

Zhang Z, Song Y, Liu C-M, Thomma BPHJ (2014) Mutational analysis of the Ve1 immune receptor that mediates Verticillium resistance in tomato. PLOS ONE 9: e99511. 


\section{Chapter 5}

\section{SUPPLEMENTAL INFORMATION}

A

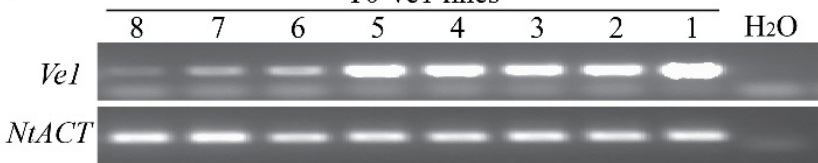

B

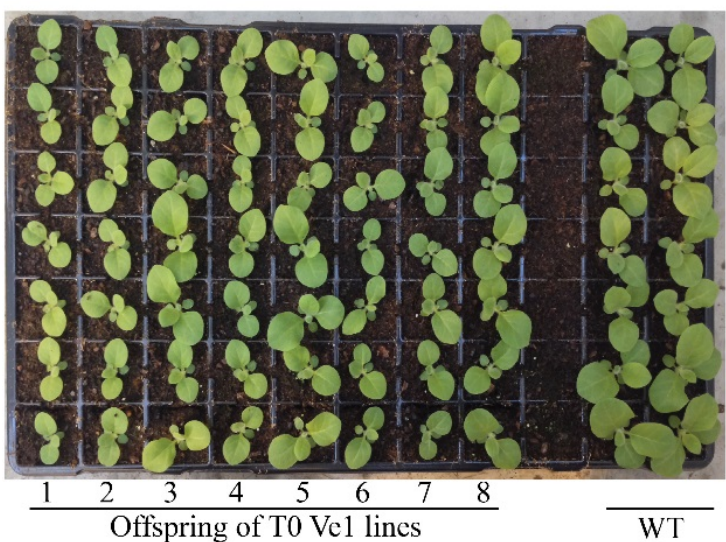

B

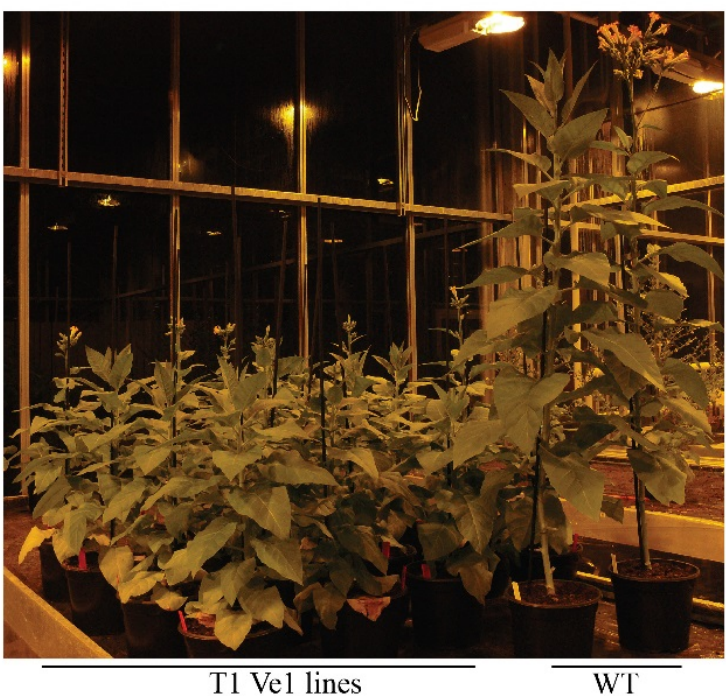

Figure S1. Characterisation of Ve1-transgenic Nicotiana tabacum cv. Samsun plants. (A) Transcripts of tomato Ve1 in eight independent T0 transformation events were detected by reverse transcription-PCR (RT-PCR). As an endogenous control, a fragment of the $N$. tabacum actin gene (NtACT) was amplified. Water was used as a PCR control. (B) Morphology of four-week-old offspring from the eight T0 transformation events and the parental line. (C) Morphology of six-month-old T1 Ve1transgenic tobacco lines and the recipient line tobacco cv. Samsun. 


\section{Ve1 confers Ave1-dependent Verticillium resistance in tobacco and cotton}
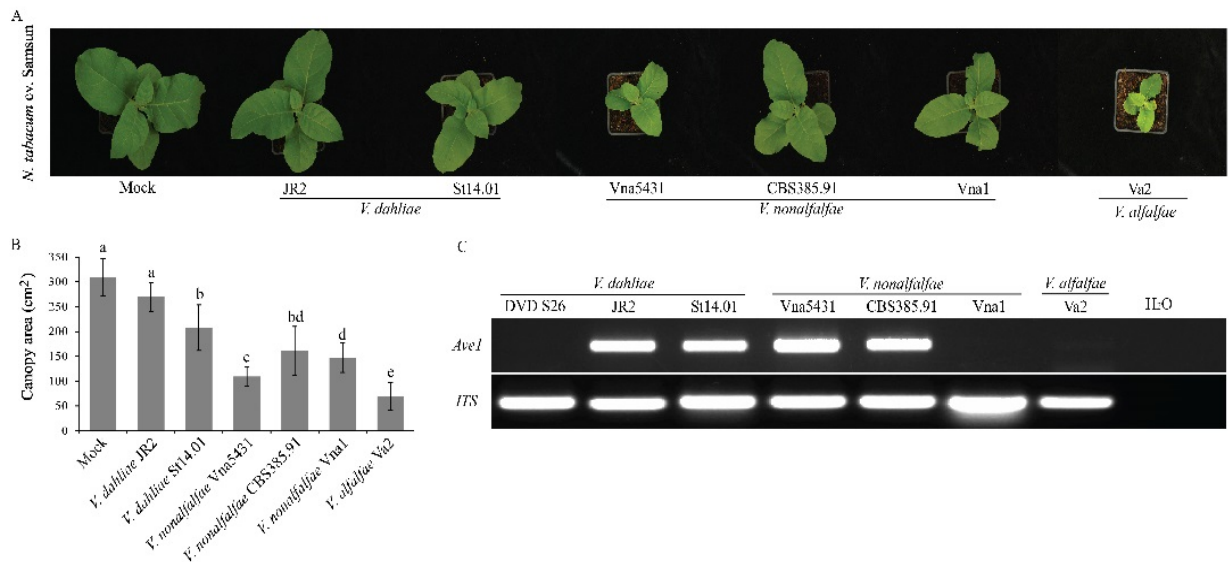

c:

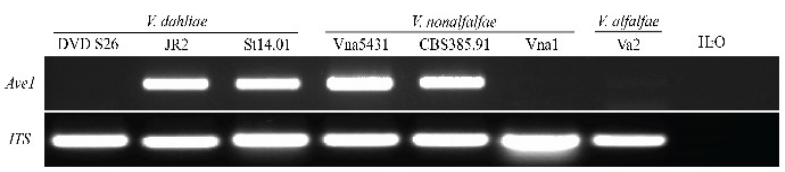

Figure S2. Verticillium strains induce differential degrees of Verticillium wilt symptoms on $\boldsymbol{N}$. tabacum cv. Samsun plants. (A) Typical appearance of tobacco cv. Samsun plants upon mockinoculation, inoculation with $V$. dahliae strains JR2 and St14.01, V. nonalfalfae strains Vna5431, CBS385.91 and Vna1, or V. alfalfae strain Va2 at 14 dpi. Inoculation experiments were performed with eight plants for each fungal strain and independently repeated twice. (B) Quantification of the canopy area of tobacco cv. Samsun plants at $14 \mathrm{dpi}$. Bars represent averages with standard deviation. Different letters indicate statistically significant differences (Student's $t$-test; $P<0.05$ ). (C) Presence of the fulllength Ave1 coding DNA sequence in different Verticillium strains was determined by PCR amplification on genomic DNA. As an endogenous control, a fragment of the Verticillium ITS region was amplified. The race 2 V. dahliae strain DVD S26 that lacks Ave1 and water were used as PCR controls. The data shown are representative of two independent experiments. 


\section{Chapter 5}

$\Lambda$

V. nonalfalfae

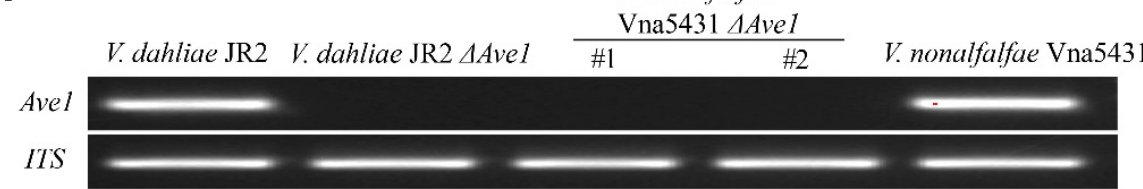

B

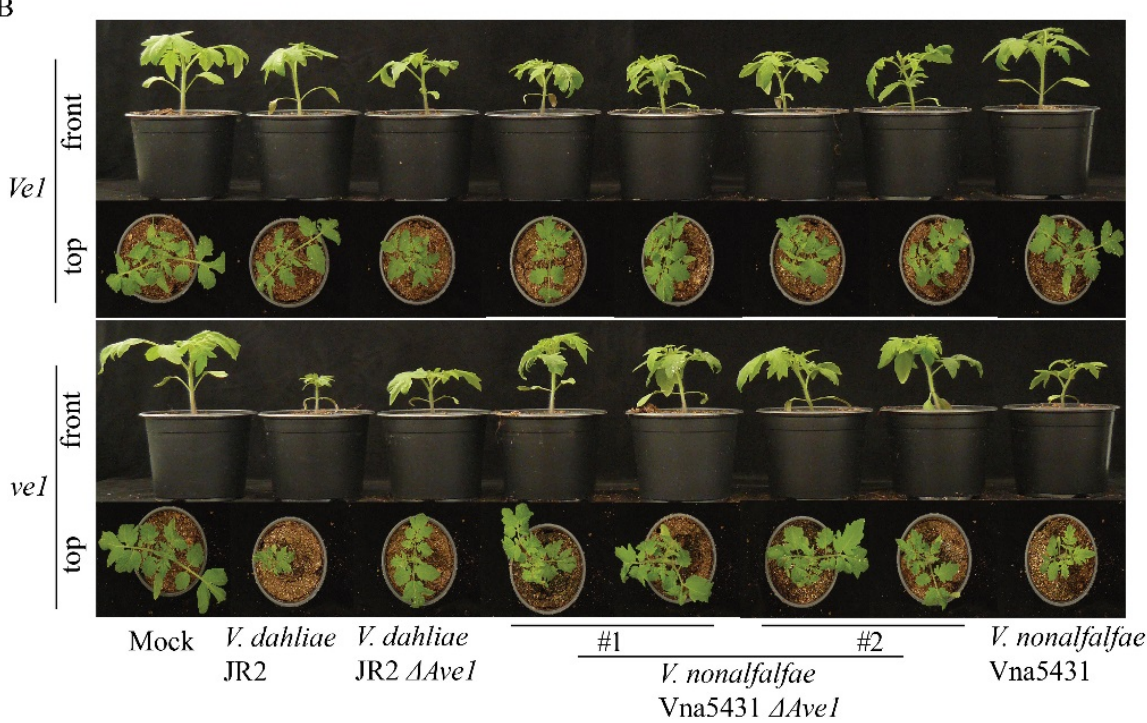

C

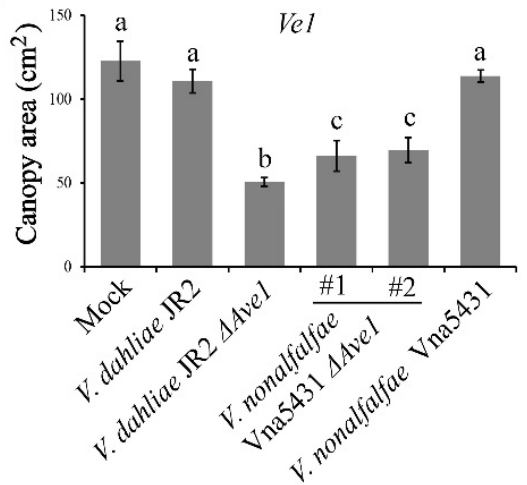

D

Figure S3. Analysis of Ave1 deletion strains of $V$. nonalfalfae Vna5431. (A) Amplification of Ave1 from genomic DNA in race $1 \mathrm{~V}$. dahliae strain JR2, an Ave1 deletion mutant of $V$. dahliae strain JR2 $(\triangle A v e 1)$, race $1 \mathrm{~V}$. nonalfalfae strain Vna5431 and two independent Ave1 deletion strains ( $\triangle$ Ave1 \#1 and \#2). As an endogenous control, a fragment of the Verticillium ITS region was amplified. (B) Typical appearance of Ve1 tomato plants (Ve1) and tomato plants lacking $V e 1$ (ve1) upon mock-inoculation or inoculation with the various Verticillium strains at 14 days post inoculation (dpi). Average canopy area of eight Ve1 (C) or ve1 (D) tomato plants inoculated with the various Verticillium strains or mockinoculation. Different letter labels indicate statistically significant differences (Student's $t$-test; $P<0.05$ ). The data shown are representative of two independent assays. 


\section{Ve1 confers Ave1-dependent Verticillium resistance in tobacco and cotton}
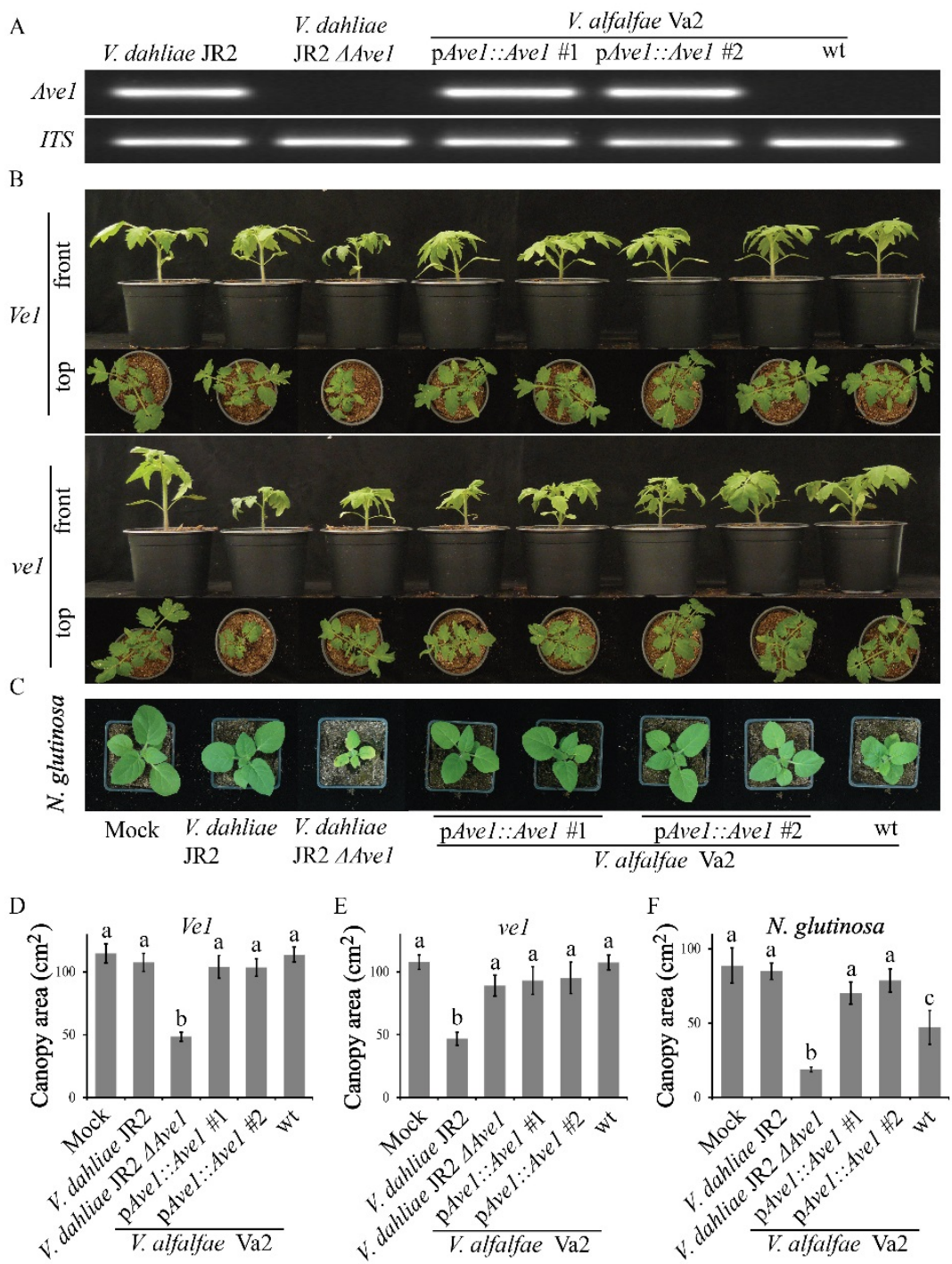

Figure S4. Analysis of ectopic expression Ave1 strains in V. alfalfae Va2. (A) Amplification of Ave1 from genomic DNA in race $1 \mathrm{~V}$. dahliae strain JR2, an Ave1 deletion mutant of $\mathrm{V}$. dahliae strain JR2 ( $\triangle$ Ave1), wild-type V. alfalfae strain Va2 (wt), and two independent Ave1 expression strains (pAve1::Ave1 $\# 1$ and \#2). As an endogenous control, a fragment of the Verticillium ITS region was amplified. (B) Typical appearance of Ve1 tomato plants (Ve1) and tomato plants lacking Ve1 (ve1) upon mockinoculation or inoculation with the various Verticillium strains at 14 dpi. (C) Typical appearance of Nicotiana glutinosa plants upon mock-inoculation or inoculation with the various Verticillium strains at 14 dpi. Average canopy area of eight Ve1 (D), ve1 (E) tomato plants, or N. glutinosa plants (F) inoculated with the various Verticillium strainsor mock-inoculation. Different letter labels indicate statistically significant differences (Student's $t$-test; $P<0.05$ ). The data shown are representative of two independent assays. 


\section{Chapter 5}

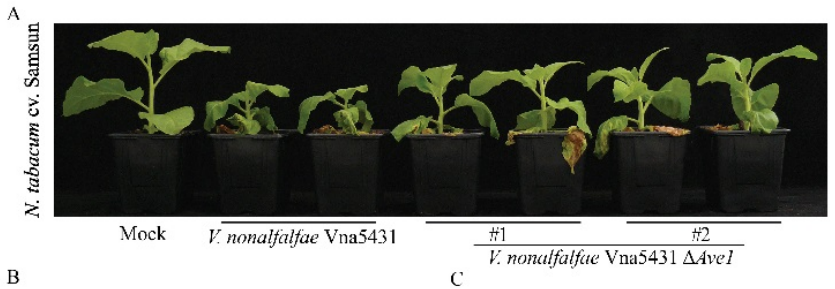

B

c
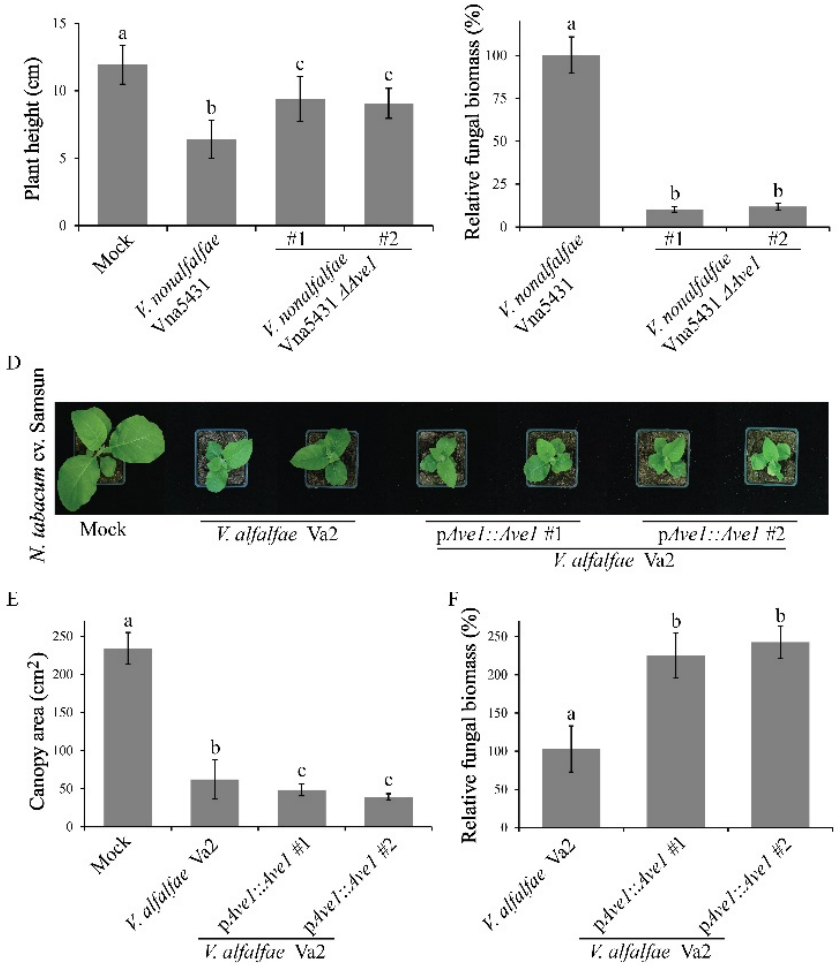

Figure S5. Ave1 acts as a virulence factor on tobacco cv. Samsun plants. (A) Typical appearance of tobacco cv. Samsun plants upon mock-inoculation, inoculation with V. nonalfalfae Vna5431, or two independent Ave1 deletion strains ( $\triangle A v e 1 \# 1$ and \#2) at 21 dpi. (B) Quantification of Verticilliuminduced plant stunting at $21 \mathrm{dpi}$. Bars represent averages with standard deviation. (C) Fungal biomass as determined with real-time PCR at $21 \mathrm{dpi}$. Bars represent Verticillium ITS levels relative to tobacco actin levels (for equilibration) with standard deviation in a sample of three pooled plants. The fungal biomass in tobacco plants upon inoculation with the wild-type $V$. nonalfalfae strain Vna5431 is set to 100\%. (D) Typical appearance of tobacco cv. Samsun plants upon mock inoculation, inoculation with $V$. alfalfae Va2, or two Ave1-experssing strains of V. alfalfae Va2 (pAve1::Ave1\#1 and \#2) at 14 dpi. (E) Quantification of in the canopy area of tobacco cv. Samsun plants at 14 dpi. Bars represent averages with standard deviation. (F) Fungal biomass as determined with real-time PCR at 14 dpi. Bars represent Verticillium ITS levels relative to tobacco actin levels (for equilibration) with standard deviation in a sample of three pooled plants. The fungal biomass in tobacco cv. Samsun plants upon inoculation with the wild-type $V$. alfalfae strain Va2 is set to $100 \%$. Different letter labels indicate statistically significant differences (Student's $t$-test; $P<0.05$ ). The data shown are representative of three independent assays. 


\section{Ve1 confers Ave1-dependent Verticillium resistance in tobacco and cotton}

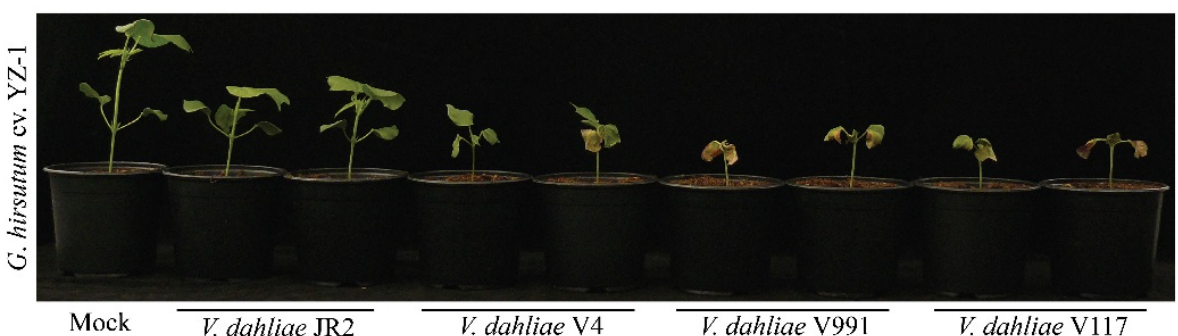

B

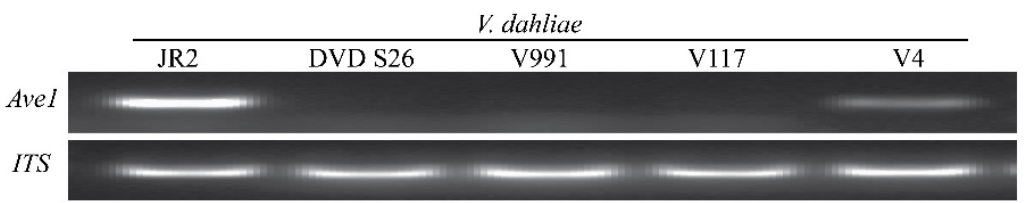

Figure S6. V. dahliae strains induce differential degrees of Verticillium wilt on cotton (Gossypium hirsutum) cv. YZ-1 plants. (A) Typical appearance of cotton cultivar YZ-1 plants upon mock-inoculation or inoculation with V. dahliae strains JR2, V4, V991 and V117 at $21 \mathrm{dpi}$. Inoculation experiments were performed with 10 plants for each $V$. dahliae strain and independently repeated twice. (B) Presence of the full-length Ave1 coding DNA sequence was determined by PCR amplification on genomic DNA. As an endogenous control, a fragment of the Verticillium ITS region was amplified. The race $2 \mathrm{~V}$. dahliae strain DVD S26 that lacks Ave1 was used as a PCR control. The data shown are representative of two independent assays. 


\section{Chapter 5}

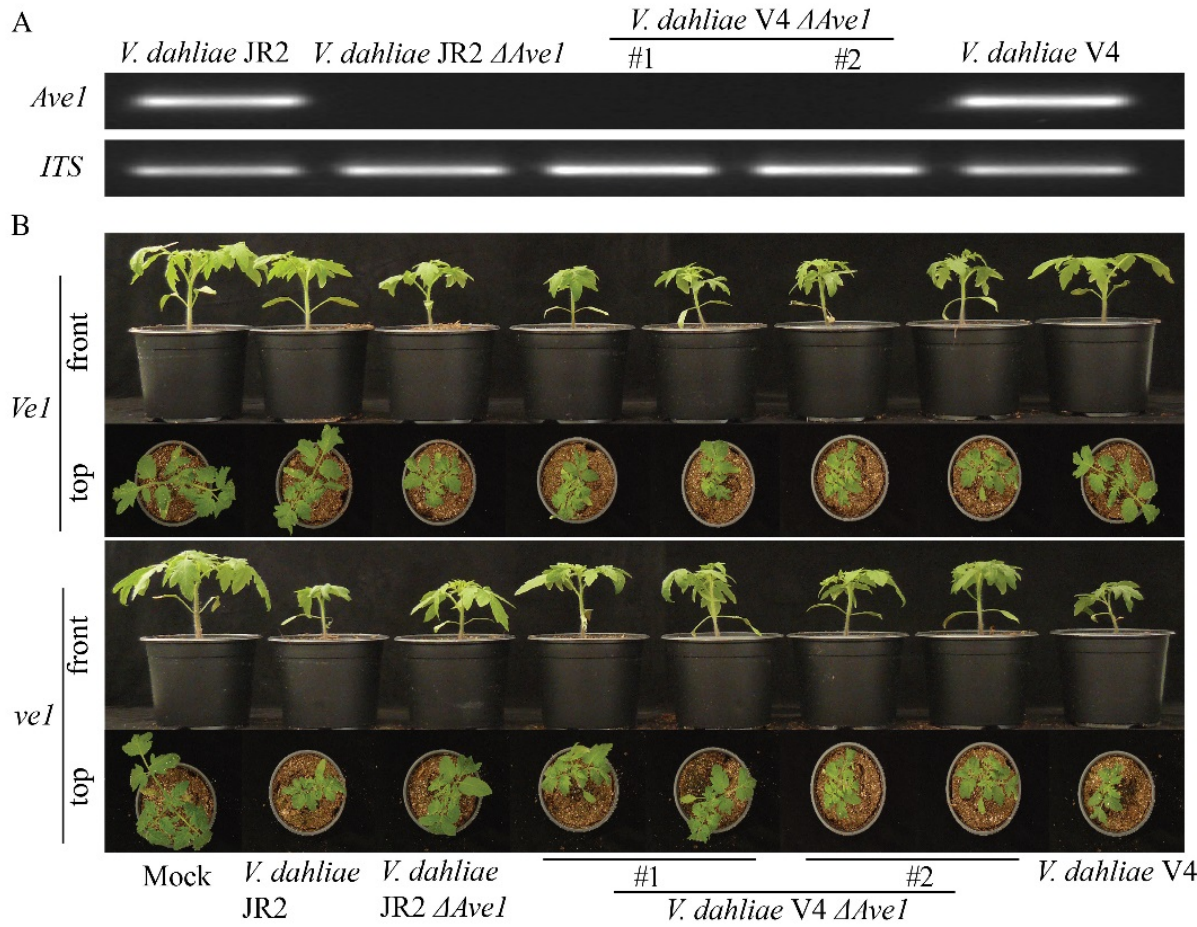

C

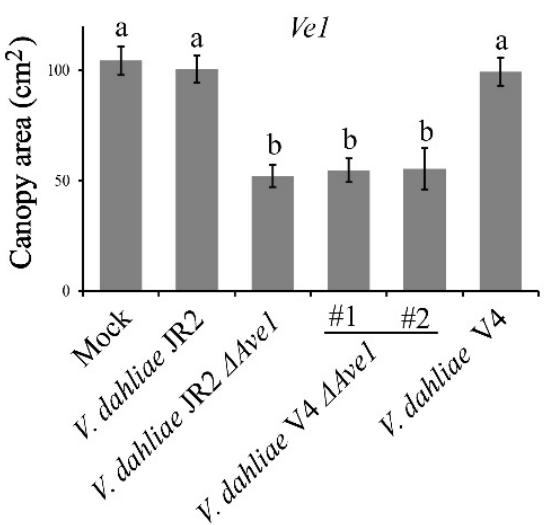

D

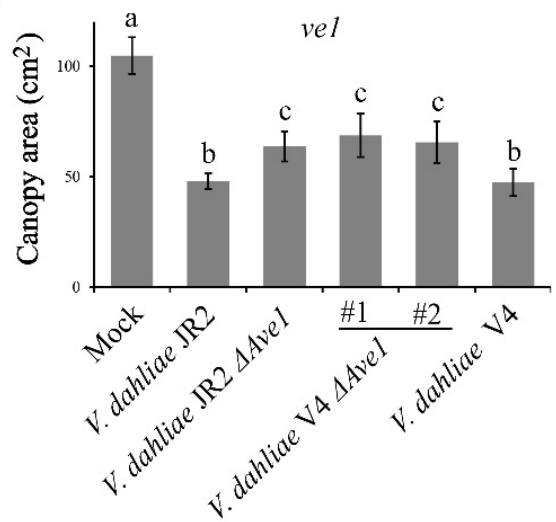

Figure S7. Analysis of Ave1 deletion strains of V. dahliae V4. (A) Amplification of Ave1 from genomic DNA in race $1 \mathrm{~V}$. dahliae strain JR2, an Ave1 deletion mutant of $V$. dahliae strain JR2 ( $\Delta$ Ave1), race $1 \mathrm{~V}$. dahliae strain V4 and two independent Ave1 deletion strains ( $\triangle A v e 1 \# 1$ and \#2). As an endogenous control, a fragment of the Verticillium ITS region was amplified. (B) Typical appearance of Ve1 tomato plants (Ve1) and tomato plants lacking Ve1 (ve1) upon mock-inoculation or inoculation with the various V. dahliae strains at $14 \mathrm{dpi}$. Average canopy area of eight Ve1 (C) ve1 (D) tomato plants inoculated with the various $V$. dahliae strains or mock-inoculation. Different letter labels indicate statistically significant differences (Student's $t$-test; $P<0.05$ ). The data shown are representative of two independent assays. 
Ve1 confers Ave1-dependent Verticillium resistance in tobacco and cotton

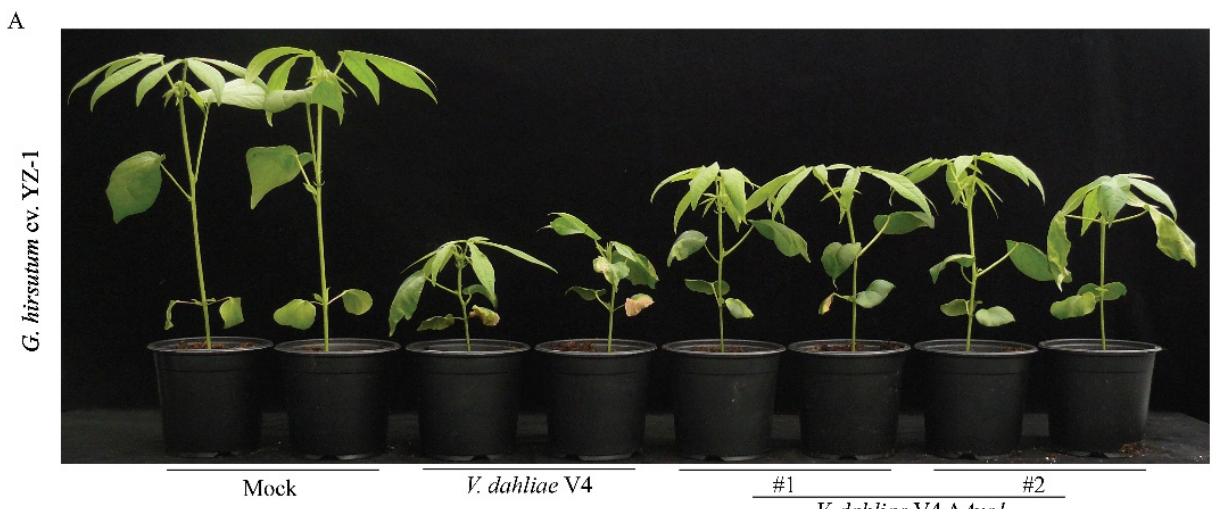

B
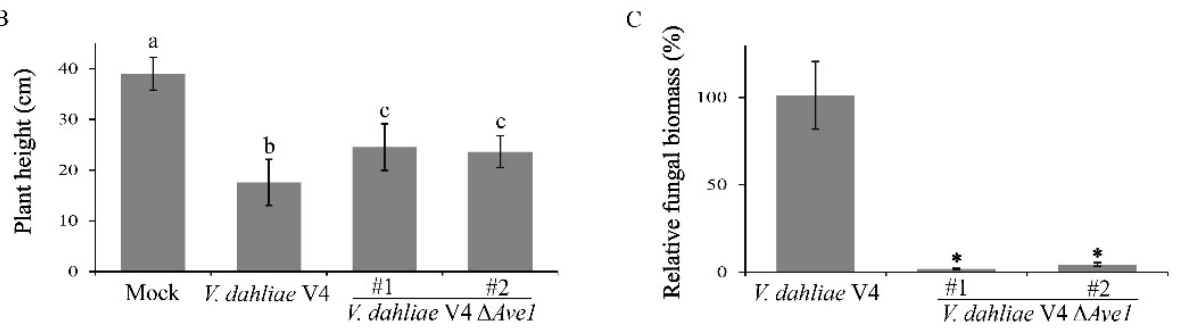

Figure S8. Ave1 acts as a virulence factor on cotton plants. (A) Typical appearance of cotton cultivar YZ-1 plants upon mock-inoculation or inoculation with $V$. dahliae V4 and two independent Ave1 deletion strains ( $\triangle$ Ave1 \#1 and \#2) at 28 dpi. (B) Quantification of Verticillium-induced plant stunting at $28 \mathrm{dpi}$. Bars represent averages with standard deviation. Different letter labels indicate statistically significant differences (Student's $t$-test; $P<0.05$ ). (C) Fungal biomass as determined with real-time PCR at 28 dpi. Bars represent Verticillium ITS levels relative to cotton ubiquitin levels (for equilibration) with standard deviation in a sample of three pooled plants. The fungal biomass in cotton plants upon inoculation with the wild-type V. dahliae strain V4 is set to $100 \%$. Asterisks indicate statistically significant differences when compared with cotton plants upon inoculation with the wild-type $V$. dahliae strain V4 (Student's $t$ test; $P<0.05$ ). The data shown are representative of three independent assays. 


\section{Chapter 5}

Table S1. Verticillium strains used in this study.

\begin{tabular}{|c|c|c|}
\hline Strain & Description & Reference \\
\hline V. dahliae JR2 & Haploid, infecting tomato; Ave1 presence & (de Jonge et al., 2012; Faino et al., 2015) \\
\hline V. dahliae St14.01 & Haploid, infecting tomato; Ave1 presence & (Fradin et al., 2009; de Jonge et al., 2012) \\
\hline V. dahliae DVD S26 & Haploid, infecting tomato; Ave1 absence & (de Jonge et al., 2012) \\
\hline V. nonalfalfae Vna5431 & $\begin{array}{l}\text { Haploid, isolated from tomato; Ave } 1 \text { presence; } \\
\text { Previously V. albo-atrum }\end{array}$ & (Fradin et al., 2009; Song et al., 2017); \\
\hline V. nonalfalfae CBS385.91 & $\begin{array}{l}\text { Haploid, isolated from tomato; Ave1 presence; } \\
\text { Previously V. albo-atrum }\end{array}$ & (Fradin et al., 2009; Song et al., 2017) \\
\hline V. nonalfalfae Vna1 & $\begin{array}{l}\text { Haploid, original host is unkown; Ave1 absence; } \\
\text { Previously V. albo-atrum }\end{array}$ & (Fradin et al., 2009; Song et al., 2017); \\
\hline V. alfalfae Va2 & $\begin{array}{l}\text { Haploid, original host is unkown; Ave1 absence; } \\
\text { Previously V. albo-atrum }\end{array}$ & (Song et al., 2017); \\
\hline V. dahliae V4 & Haploid, infecting cotton; Ave1 presence & (López-Escudero et al., 2004; Song et al., 2017) \\
\hline V. dahliae V117 & Haploid, infecting cotton; Ave1 absence & (López-Escudero et al., 2004; Song et al., 2017) \\
\hline V. dahliae V991 & Haploid, infecting cotton; Ave1 absence & (Xu et al., 2014) \\
\hline V. dahliae JR2 $\triangle$ Ave1 & Ave1 deletion mutant in $V$. dahliae JR2 & (de Jonge et al., 2012) \\
\hline $\begin{array}{l}\text { V. nonalfalfae } \\
\text { Vna5431 } 4 \text { Ave1 \#1 }\end{array}$ & $\begin{array}{l}\text { Ave1 deletion mutant in } V . \text { nonalfalfae Vna5431, } \\
\text { colony } 1\end{array}$ & This study \\
\hline $\begin{array}{l}\text { V. nonalfalfae } \\
\text { Vna5431 } \triangle \text { Ave1 \#2 }\end{array}$ & $\begin{array}{l}\text { Ave1 deletion mutant in V. nonalfalfae Vna5431, } \\
\text { colony } 2\end{array}$ & This study \\
\hline $\begin{array}{l}\text { V. alfalfae } \mathrm{Va} 2 \\
\text { pAve1::Ave1 } \# 1\end{array}$ & V. alfalfae strain Va2 expressing Ave1, colony 1 & This study \\
\hline $\begin{array}{l}\text { V. alfalfae } \mathrm{Va} 2 \\
\text { pAve1::Ave1 \#2 }\end{array}$ & V. alfalfae strain Va2 expressing Ave1, colony 2 & This study \\
\hline V. dahliae V4 $\Delta$ Ave1 \#1 & Ave 1 deletion mutant in $V$. dahliae $\mathrm{V} 4$, colony 1 & This study \\
\hline V. dahliae V4 $\Delta$ Ave1 \#2 & Ave1 deletion mutant in $V$. dahliae $\mathrm{V} 4$, colony 2 & This study \\
\hline
\end{tabular}


Ve1 confers Ave1-dependent Verticillium resistance in tobacco and cotton

Table S2. Primers used in this study.

\begin{tabular}{|c|c|c|}
\hline Primer name & Oligonucleotide sequence $\left(5^{\prime} \rightarrow 3^{\prime}\right)$ & Description $^{a}$ \\
\hline Ve1-F(PCR) & CATATTGAAATTAGCGTCTTGTCGG & RT-PCR; Ve1 expression in tobacco \\
\hline Ve1-R(PCR) & ACCGAGAAAAAGGAGGCAAAAC & RT-PCR; Ve1 expression in tobacco \\
\hline Ave1-F & CACCATGAAGCTTTCTACGCTTGGAG & Ave1; PCR \\
\hline Ave1-R & TTATATCTGTCTAAATTCGATGTTGAC & Ave1; PCR \\
\hline ITS-F & AAAGTTTTAATGGTTCGCTAAGA & Verticillium ribosomal internal transcribed spacer region (ITS) \\
\hline ITS-R & CTTGGTCATTTAGAGGAAGTAA & Verticillium ribosomal internal transcribed spacer region (ITS) \\
\hline NtACT-F & CTATTCTCCGCTTTGGACTTGGCA & Tobacco actin \\
\hline NtACT-R & AGGACCTCAGGACAACGGAAACG & Tobacco actin \\
\hline GhUb-F & GAAGGCATTCCACCTGACCAAC & Cotton ubiquitin \\
\hline GhUb-R & CAAAACTCСAAAATCATACCСAAAG & Cotton ubiquitin \\
\hline Ve1-F(RT) & ATGGTTGCTGATGATTATGTGG & RT-PCR; Ve1 expression in cotton \\
\hline Ve1-R(RT) & AATCAGGCAATGGTGTAGGTG & RT-PCR; Ve1 expression in cotton \\
\hline
\end{tabular}

aThe type of experiment for which the primers were used is indicated in brackets (RT-PCR: Reverse Transcription-PCR).

\section{REFERENCES}

de Jonge R, van Esse HP, Maruthachalam K, Bolton MD, Santhanam P, Saber MK, Zhang Z, Usami T, Lievens B, Subbarao KV, Thomma BPHJ (2012) Tomato immune receptor Ve1 recognizes effector of multiple fungal pathogens uncovered by genome and RNA sequencing. Proc Natl Acad Sci USA 109: 5110-5115.

Faino L, Seidl MF, Datema E, van den Berg GC, Janssen A, Wittenberg AH, Thomma BPHJ (2015) Single-molecule real-time sequencing combined with optical mapping yields completely finished fungal genome. mBio 6: e00936-00915.

Fradin EF, Zhang Z, Ayala JCJ, Castroverde CD, Nazar RN, Robb J, Liu C-M, Thomma BPHJ (2009) Genetic dissection of Verticillium wilt resistance mediated by tomato Ve1. Plant Physiol 150: 320332.

López-Escudero FJ, del Río C, Caballero JM, Blanco-López MA (2004) Evaluation of olive cultivars for resistance to Verticillium dahliae. Eur J Plant Pathol 110: 79-85.

Song Y, Zhang Z, Boshoven J, Rovenich H, Seidl M, Jakse J, Maruthachalam K, Liu C-M, Subbarao K, Javornik B, Thomma BPHJ (2017) Tomato immune receptor Ve1 recognizes surface-exposed colocalized N- and C-termini of Verticillium dahliae effector Ave1. bioRxiv https://doi.org/10.1101/103473.

Xu L, Zhang W, He X, Liu M, Zhang K, Shaban M, Sun L, Zhu J, Luo Y, Yuan D, Zhang X, Zhu L (2014) Functional characterization of cotton genes responsive to Verticillium dahliae through bioinformatics and reverse genetics strategies. J Exp Bot 65: 6679-6692. 



\section{Chapter 6}

\section{Host-induced gene silencing compromises Verticillium wilt in tomato and Arabidopsis}

Yin Song and Bart P.H.J. Thomma

Laboratory of Phytopathology, Wageningen University, Droevendaalsesteeg 1, 6708 PB Wageningen, the Netherlands

This chapter has been published as:

Song Y, Thomma BPHJ (2016) Host-induced gene silencing compromises Verticillium wilt in tomato and Arabidopsis. Mol Plant Pathol DOI: 10.1111/mpp.12500. 


\section{Chapter 6}

\section{ABSTRACT}

Verticillium wilt, caused by soil-borne fungi of the genus Verticillium, is an economically important disease that affects a wide range of host plants. Unfortunately, host resistance against Verticillium wilts is not available for many plant species, and the disease is notoriously difficult to combat. Host-induced gene silencing (HIGS) is an RNA interference (RNAi) based process in which small RNAs are produced by the host plant to target parasite transcripts. HIGS has emerged as a promising strategy for improving plant resistance against pathogens by silencing genes that are essential for these pathogens. Here, we assessed whether HIGS can be utilized to suppress Verticillium wilt disease by silencing three previously identified virulence genes of V. dahliae (encoding Ave1, Sge1 and NLP1) through the host plants tomato and Arabidopsis. In transient assays, tomato plants were agroinfiltrated with Tobacco rattle virus (TRV) constructs to target $V$. dahliae transcripts. Subsequent V. dahliae inoculation revealed suppression of Verticillium wilt disease upon treatment only with one of the three TRV constructs. Next, expression of RNAi constructs targeting transcripts of the same three $V$. dahliae virulence genes was pursued in stable transgenic Arabidopsis thaliana plants. In this host, $V$. dahliae inoculation revealed reduced Verticillium wilt disease in for two out of three targets. Thus, our study suggests that, depending on the target gene chosen, HIGS against $V$. dahliae is operational in tomato and $A$. thaliana plants and may be exploited to engineer resistance in Verticillium wilt-susceptible crops. 


\section{INTRODUCTION}

Verticillium wilts are vascular wilt diseases that are caused by soil-borne fungi of the genus Verticillium (Fradin and Thomma, 2006; Klimes et al., 2015). This genus comprises ten species of soil-borne fungi that differ in their morphological features, such as resting structures, as well as in their ability to cause plant diseases (Inderbitzin et al., 2011). Within the Verticillium genus, V. dahliae is the most notorious pathogenic species that can infect hundreds of dicotyledonous hosts, including ecologically important plants and many high-value crops worldwide (Fradin and Thomma, 2006; Klosterman et al., 2009). Verticillium wilt diseases are difficult to control due to the long viability of the resting structures, the wide host range of the pathogens, and the inability of fungicides to affect the pathogen once in the plant vascular system. Thus, the most sustainable way to control Verticillium wilt diseases is the use of resistant cultivars. Polygenic resistance to Verticillium spp. has been described for several plant species, including potato, hop, alfalfa, cotton and strawberry ( Antanaviciute et al., 2015; Bolek et al., 2005; Jakse et al., 2013; Simko et al., 2004; Wang et al., 2008; Yang et al., 2008;), whereas single dominant resistance genes have been identified only in tomato, sunflower, cotton, potato and lettuce (Barrow, 1970; Christopoulou et al., 2015; Hayes et al., 2011; Lynch et al., 1997; Mert et al., 2005; Putt, 1964; Schaible et al., 1951). In tomato (Solanum lycopersicum), a single dominant locus that confers Verticillium resistance has been identified as the Ve locus, which controls Verticillium isolates that are assigned to race 1, whereas race 2 strains escape recognition (Pegg, 1974; Schaible et al., 1951). The $V e$ locus contains two closely linked and inversely oriented genes, $V e 1$ and Ve2, both of which encode extracellular leucine rich repeat (eLRR) receptor-like proteins (RLPs) (Kawchuk et al., 2001; Wang et al., 2010). Of these, only Ve1 was found to confer resistance against race 1 isolates of Verticillium in tomato (Fradin et al., 2009). Interestingly, interfamily transfer of Ve1 from tomato to Arabidopsis thaliana has resulted in race-specific Verticillium resistance in the latter species (Fradin et al., 2011, 2014; Zhang et al., 2014), implying that the underlying immune signaling pathway is conserved (Fradin et al., 2011; Thomma et al., 2011). Tomato Ve1 serves as an immune receptor for recognition of the effector protein Ave 1 that is secreted by race 1 strains of $V$. dahliae (de Jonge et al., 2012). More recently, homologs of tomato $\mathrm{Ve} 1$ acting as immune receptors that govern 


\section{Chapter 6}

resistance against $V$. dahliae race 1 strains through recognition of the Ave1 effector have been characterized in other plant species including tobacco, potato, wild eggplant and hop, suggesting an ancient origin of the immune receptor Ve1 (Song et al., 2016).

Although the tomato Ve1 gene is still currently deployed in tomato cultivars, isolates of Verticillium that escape Ve1-mediated recognition appeared within a few years after the introduction of the tomato Ve1 (Pegg and Brady, 2002). These race 2 isolates of Verticillium steadily supplanted race 1 strains in various regions because of the extensive use of Verticillium race 1-resistant cultivars (Dobinson et al., 1996). Currently, no source of commercially employed resistance to Verticillium race 2 strains has been described.

RNA interference (RNAi) is a conserved regulatory mechanism that affects gene expression in eukaryotic organisms (Baulcombe, 2005). RNA silencing is triggered by the processing of double stranded RNA (dsRNA) precursors into short interfering RNA (siRNAs) duplexes of 21-28 nucleotides in length, and followed by the guided cleavage or translational repression of sequence-complementary singlestranded RNAs by the generated siRNAs duplexes, which are incorporated into a silencing complex called RISC (RNA-induced silencing complex) (Ruiz-Ferrer and Voinnet, 2009). Plants and other eukaryotes have evolved RNAi machineries that not only regulate developmental programs, but also provide protection from invaders, such as viruses. In plants, RNAi has been exploited extensively and has become a powerful functional genomics tool to silence the expression of genes of interest as well as to engineer viral resistance (Duan et al., 2012). Interestingly, organisms that live within, or develop intimate contact with, a host, such as bacteria (Escobar et al., 2001; 2002), nematodes (Huang et al., 2006), insects (Baum et al., 2007; Mao et al., 2007) and parasitic plants (Tomilov et al., 2008), are sensitive to small RNAs generated by the host and that are targeted to parasite transcripts. This so-called host-induced gene silencing (HIGS) has also emerged as a promising strategy against plant pathogens, including fungi and oomycetes. Initial reports of HIGS against filamentous pathogens were described for the maize kernel and ear rot pathogen Fusarium verticillioides (Tinoco et al., 2010) and the barley powdery mildew fungus Blumeria graminis (Nowara et al., 2010). Subsequent reports demonstrated the functionality of HIGS in suppressing diseases caused by the 
fungal pathogens Puccinia spp. (Panwar et al., 2013; Yin et al., 2011; 2015; Zhang et al., 2012), Fusarium spp. (Koch et al., 2013; Ghag et al., 2014; Cheng et al., 2015; Hu et al., 2015; Chen et al., 2016), Sclerotinia sclerotiorum (Andrade et al., 2015) and Rhizoctonia solani (Zhou et al., 2016), as well as by the oomycete pathogens Phytophthora infestans (Jahan et al., 2015; Sanju et al., 2015) and Bremia lactucae (Govindarajulu et al., 2015). Many of these pathogens make very intimate contact with host cells, potentially facilitating the occurrence of HIGS. In this study, we assessed whether HIGS can be used to suppress Verticillium wilt disease in tomato and $A$. thaliana by targeting previously identified virulence factors of $V$. dahliae.

\section{RESULTS}

Tobacco rattle virus-based silencing in tomato compromises V. dahliae Ave1 expression

Tobacco rattle virus (TRV)-based virus-induced gene silencing (VIGS) has extensively been used in various plant species, including tomato (Liu et al., 2002; Senthil-Kumar et al., 2007), and TRV-based VIGS has successfully been used to investigate candidate genes for their involvement in Verticillium wilt resistance in tomato (Fradin et al., 2009). In order to investigate whether HIGS can be established against the xylem-colonizing fungus $V$. dahliae, we attempted to exploit TRV-based VIGS to produce dsRNAs that are targeted towards V. dahliae Ave1 transcripts. The experiment was performed in $V e 1$ tomato plants that are normally immune to infection by Ave1-carrying $V$. dahliae strains, such that successful HIGS would immediately result in vascular wilt disease that does not occur if Ave1 expression is not compromised (Fradin et al., 2009; de Jonge et al., 2012). To this end, a 1:1 mixture of Agrobacterium tumefaciens cultures carrying TRV1 and TRV2::Ave1 (Figure 1A) was infiltrated into cotyledons of Ve1 tomato plants. A recombinant construct containing a fragment of the GUS gene (TRV2::GUS) was used as a negative control (Figure $1 \mathrm{~A}$ ). At ten days after TRV treatment, plants were challenged with either the V. dahliae race 1 strain JR2 (Faino et al., 2015), or an Ave1 deletion mutant ( $V$. dahliae JR2 $\triangle A v e 1$; de Jonge et al., 2012), and inspected for Verticillium wilt symptoms (stunting and wilting) up to 14 days post inoculation (dpi). As expected, no significant disease symptoms were observed on TRV::GUStreated plants that were inoculated with the wild-type race $1 \mathrm{~V}$. dahliae strain 


\section{Chapter 6}

A

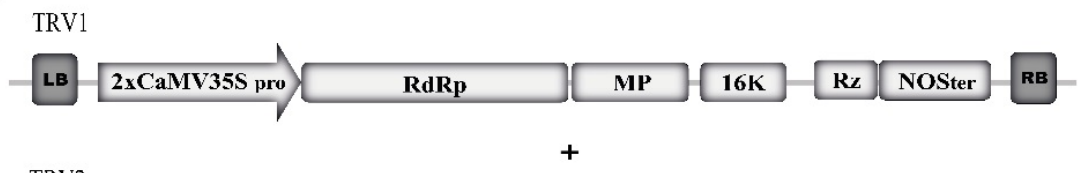

TRV2

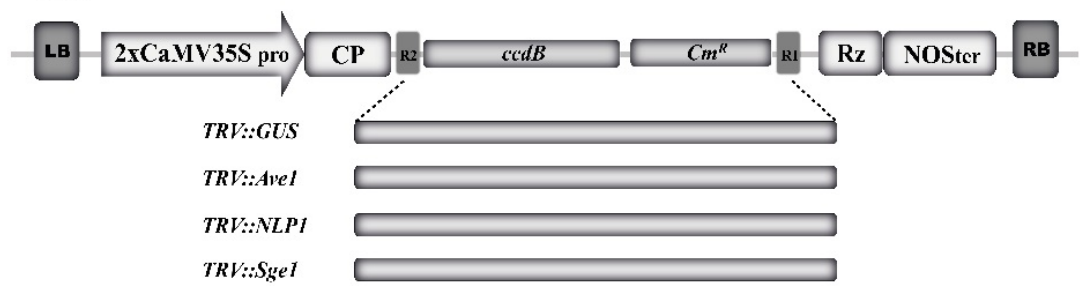

B

pFAST R03

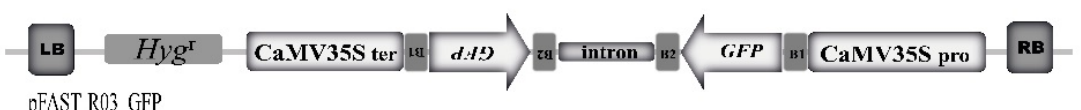

pFAST R03 GFP

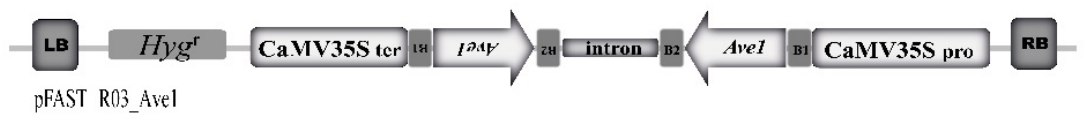

pHellsgate 12

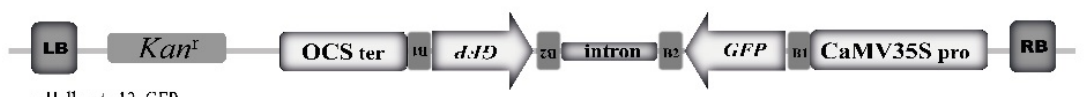

pHellsgate 12_GFP

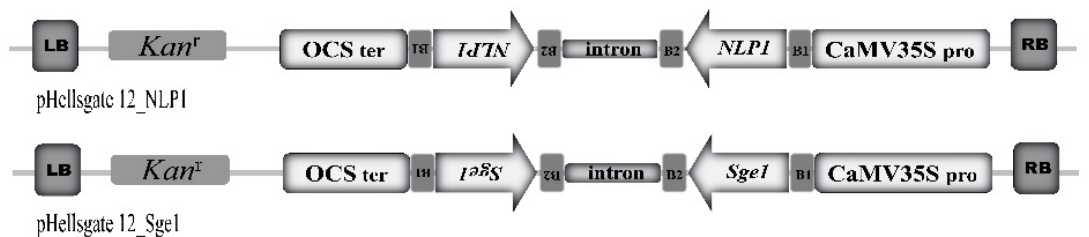

Figure 1. Schematic organization of the T-DNA region of the binary vectors used for gene silencing. (A) Schematic representation of the T-DNA region of the Tobacco rattle virus (TRV)-based virus-induced gene silencing (VIGS) vectors. Verticillium dahliae Ave1, Sge1 and NLP1 DNA fragments were inserted between the double CaMV35S promoter (2X35 CaMV35Spro) and the nopaline synthase gene terminator (NOSter) in the TRV2 vector to generate the TRV-based fungal gene silencing vectors TRV::Ave1, TRV::Sge1 and TRV::NLP1, respectively. Control construct TRV::GUS was described earlier (Song et al., 2016). RdRp, RNA-dependent RNA polymerase; 16K, 16 kDa cysteine-rich protein; MP, movement protein; $\mathrm{CP}$, coat protein; Rz, self-cleaving ribozyme; $c c d B$, negative selection marker used in bacteria; $\mathrm{Cm}^{R}$, chloramphenicol resistance marker; R1 and R2, attR1 and attR2 sites. (B) Schematic diagrams of the T-DNA region of the binary vectors generated for producing a hairpin RNA of Verticillium genes Ave1 (pFAST R03_Ave1), NLP1 (pHellsgate 12_NLP1) and Sge1 (pHellsgate 12_Sge1), as well as the green fluorescent protein gene (pFAST R03_GFP and pHellsgate 12_GFP) in transgenic A. thaliana plants. CaMV35Spro, CaMV35S promoter; CaMV35Ster, CaMV35S terminator; OCSter, octopine synthase gene terminator; $\mathrm{Hyg}^{r}$, hygromycin resistance gene; $\mathrm{Kan}^{r}$, kanamycin resistance gene; B1 and B2, attB1 and attB2 sites. LB and RB, left and right borders of T-DNA. 
(Figure 2A), indicating that TRV treatment by itself does not compromise Ave1triggered immunity in Ve1 tomato plants. Furthermore, the Ave1 deletion mutant caused clear Verticillium wilt disease, as Verticillium wilt disease developed on Ve1 plants treated with TRV2::Ave1 and subsequent inoculation with the Ave1 deletion strain (Figure 2A). However, intriguingly, Verticillium wilt disease also developed on Ve1 plants upon TRV2::Ave1 treatment and subsequent inoculation with the wildtype race $1 \mathrm{~V}$. dahliae strain (Figure 2A). This finding suggests that Ave1 expression in $V$. dahliae is indeed compromised due to TRV-induced HIGS in tomato. The compromised immunity was confirmed by fungal recovery assays by plating stem sections on potato dextrose agar (PDA) plates, and by fungal biomass quantification in stem sections of the inoculated plants (Figure 2).
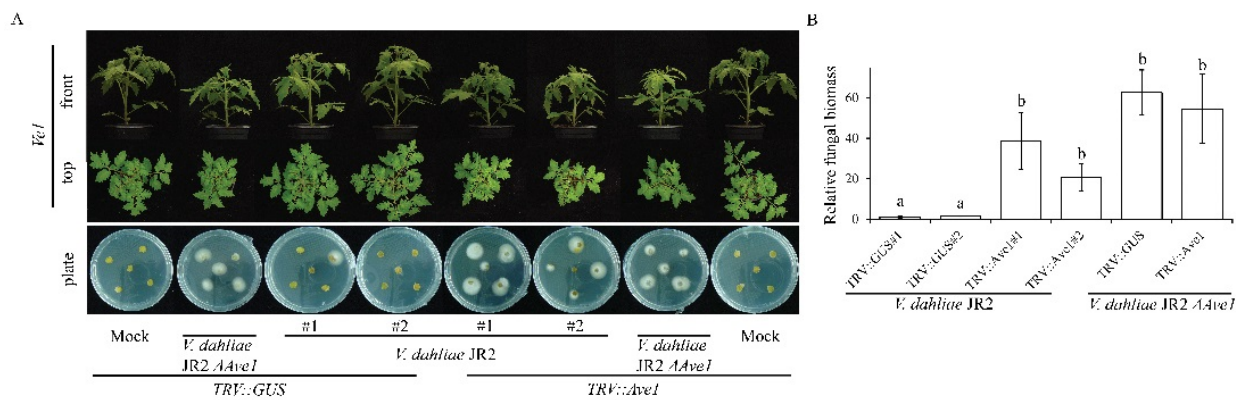

Figure 2. TRV-mediated fungal gene silencing in tomato plants compromises Verticillium dahliae Ave1 expression. (A) Upon inoculation with the wild-type race $1 \mathrm{~V}$. dahliae strain JR2, the impairment of Ave1-triggerred immunity in Ve1 tomato plants treated with TRV::Ave1 when compared with the TRV::GUS-treated plants evidenced by stunted Ve1 plants at 14 days post inoculation (14 dpi) and fungal outgrowth upon plating stem sections on potato dextrose agar (PDA). The Ave1 deletion mutant (V. dahliae JR2 $\triangle A v e 1$ ) was used as Verticillium inoculation control. Plants were photographed at 14 dpi. (B) Fungal biomass was determined by qPCR in Verticillium-inoculated Ve1 plants at 14 dpi. Bars represent Verticillium ITS levels relative to tomato actin levels (for equilibration) with standard deviation in a sample of three pooled plants. The fungal biomass in Ve1 tomato plants upon TRV::GUS treatment and subsequent inoculation with the wild-type race $1 \mathrm{~V}$. dahliae strain is set to 1 . Different letter labels indicate significant differences $(P<0.05)$. The data shown are representative of three independent experiments.

It was recently demonstrated that Tobacco mosaic virus (TMV) may infect fungi in addition to plants, remaining for up to six subcultures, and also persisted in plants infected by the virus-infected fungus (Mascia et al., 2014). This finding raises 


\section{Chapter 6}

the theoretical possibility that also TRV may infect $V$. dahliae and cause VIGS (directly) rather than HIGS from inside the tomato cells (indirectly). To exclude that the impairment of Ave1-triggered immunity in Ve1 tomato plants is due to TRVinfection of $V$. dahliae itself, stem sections from Verticillium-inoculated TRV::GUSand TRV::Ave1-treated tomato plants were placed on PDA plates. A single colony that grew from wild-type $V$. dahliae-inoculated TRV::GUS-treated plants (V. dahliae JR2 ${ }^{\text {TRV:GUS }}$ ) and Ave1 deletion mutant-inoculated TRV::Ave1-treated plants (V. dahliae JR2 $\Delta$ Ave1 ${ }^{T R V: A v e 1}$ ), and three independent colonies that grew from wild-type $V$. dahliae-inoculated TRV::Ave1-treated plants (V. dahliae JR2 ${ }^{\text {TRV::Ave1 }}$ ) were subjected to PCR to detect a viral coat protein gene fragment of TRV (TRV2_CP), showing that TRV2_CP was not detected in all fungal isolates (Figure S1A). Furthermore, the fungal isolates were used to infect $V e 1$ tomato plants and tomato plants that lack Ve1. This analysis showed that, similar to $V$. dahliae JR2TRV:GUS, also V. dahliae JR2 ${ }^{\text {TRV:Ave1 }}$ induced no disease symptoms on tomato plants expressing Ve1, while tomato plants lacking Ve1 showed clear Verticillium wilt disease (Figure S1B-D). These data support the hypothesis that the impairment of Ave1-triggered immunity in Ve1 plants is not caused by TRV-infection of $V$. dahliae, but genuinely by HIGS through TRV-treatment of tomato.

\section{TRV-based fungal gene silencing in tomato inhibits Verticillium wilt disease}

To further investigate the potential of TRV-mediated HIGS against $V$. dahliae in tomato, two previously identified virulence genes of $V$. dahliae were targeted. The first target gene is NLP1, encoding a member of the necrosis- and ethyleneinducing-like protein (NLP) family in V. dahliae, and targeted deletion of NLP1 in $V$. dahliae significantly compromises virulence on tomato as well as on A. thaliana plants (Santhanam et al., 2013). The second candidate gene is V. dahliae Sge1,

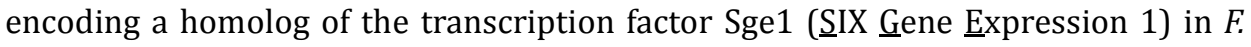
oxsporum, and $V$. dahliae mutants of the Sge1 are non-pathogenic on tomato (Santhanam and Thomma, 2013).

To produce dsRNAs of the gene fragments in planta, cotyledons of ten-day-old Moneymaker tomato plants were treated with the silencing constructs TRV::GUS, TRV2::NLP1 and TRV2::Sge1 in combination with TRV1 (Figure 1A), respectively. At ten days after TRV treatment, plants were challenged with either the $V$. dahliae 
strain JR2 (Faino et al., 2015), a NLP1 deletion mutant (V. dahliae JR2 $\triangle N L P 1$; Santhanam et al., 2013), or a Sge1 deletion mutant (V. dahliae JR2 $\Delta$ Sge1; Santhanam and Thomma, 2013), and monitored for Verticillium wilt symptoms on tomato plants at $14 \mathrm{dpi}$. As expected, significantly compromised Verticillium wilt symptoms were observed on Moneymaker tomato plants upon TRV2::GUS or TRV2::NLP1

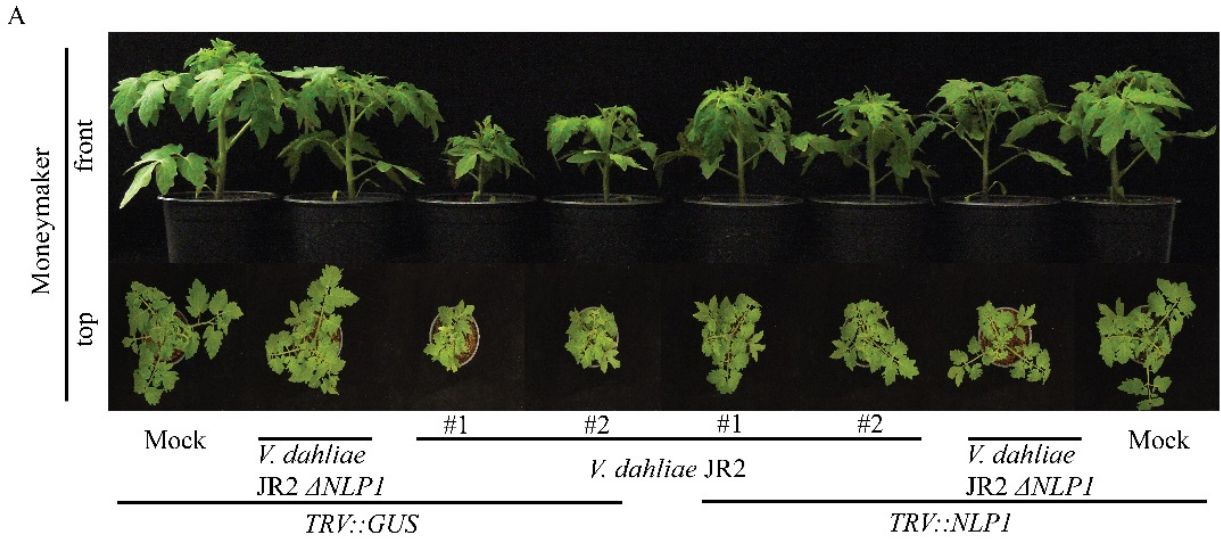

B

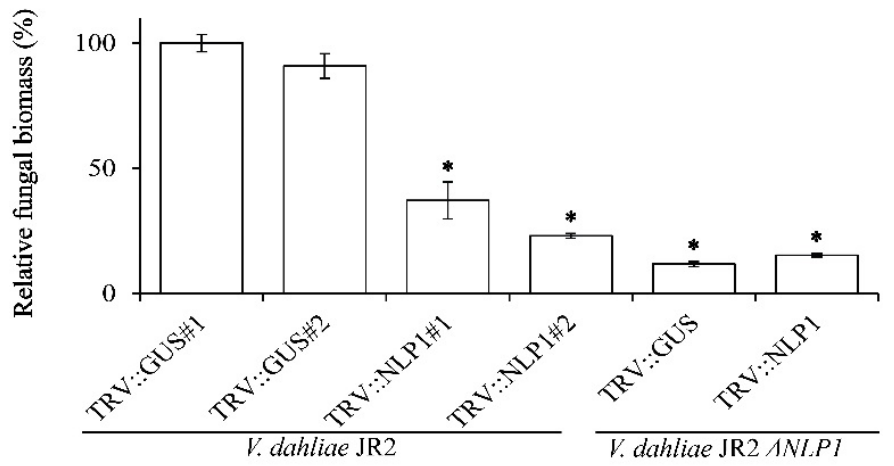

Figure 3. Effect of TRV-mediated NLP1 silencing in Moneymaker tomato plants on Verticillium dahliae inoculation. (A) Agroinfiltration with the $T R V:: N L P 1$ construct resulted in the suppression of Verticillium wilt symptoms on tomato plants, whereas no effect on disease development was observed on plants treated with the TRV::GUS. The NLP1 deletion mutant (V. dahliae JR2 $N$ NLP1) was used as Verticillium inoculation control. Plants were photographed at $14 \mathrm{dpi}$. (B) Fungal biomass was determined by qPCR in Verticillium-inoculated Moneymaker tomato plants at 14 dpi. Bars represent Verticillium ITS levels relative to tomato actin levels (for equilibration) with standard deviation in a sample of three pooled plants. The fungal biomass in tomato plants upon TRV::GUS treatment and subsequent inoculation with the wild-type $V$. dahliae strain JR2 is set to $100 \%$ (control). Asterisks indicate significant differences when compared with the TRV::GUS-treated plants upon inoculation with the $V$. dahliae strain JR2 $(P<0.05)$. The data shown are representative of three independent experiments. 


\section{Chapter 6}

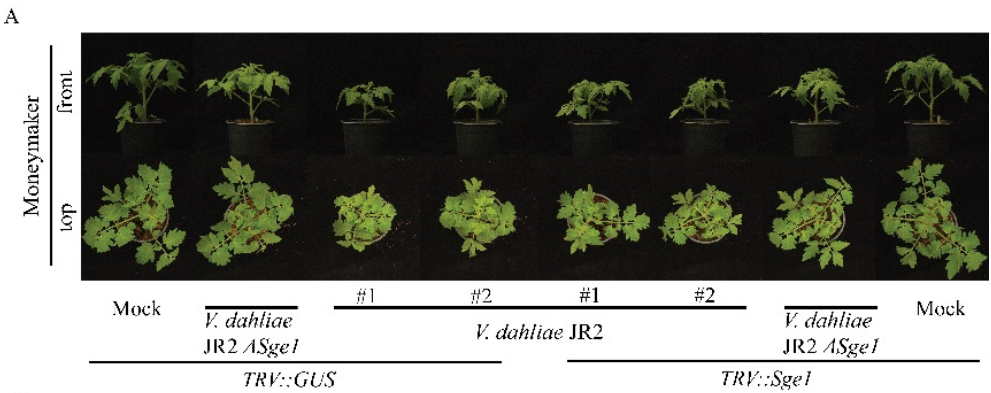

B
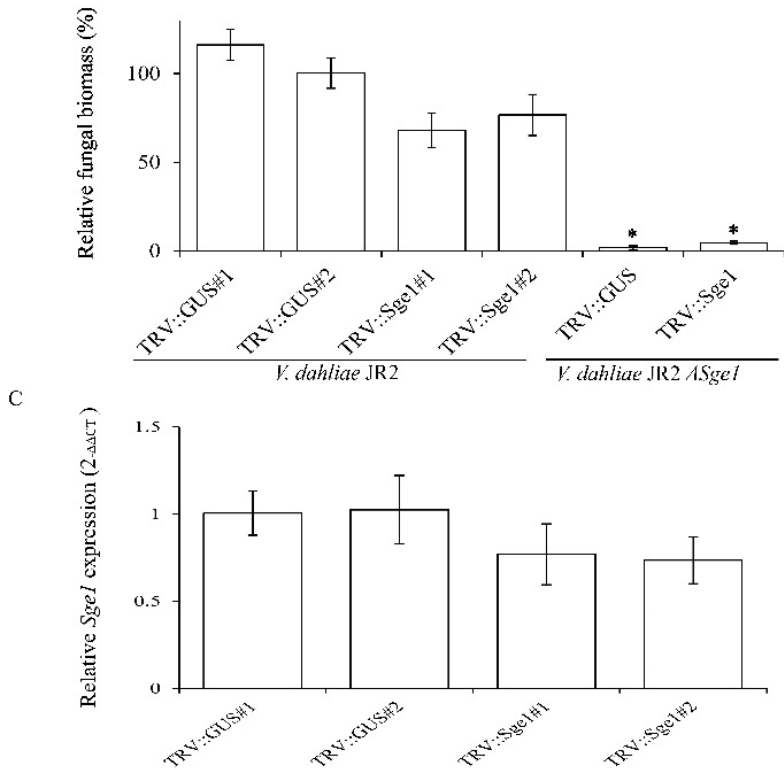

Figure 4. Effect of TRV-mediated Sge1 silencing in tomato plants on Verticillium dahliae inoculation. (A) Upon inoculation with the V. dahliae strain JR2, no effect on disease development was observed on TRV::Sge1-treated plants compared to TRV::GUS-treated plants. The Sge1 deletion mutant (V. dahliae JR2 4 Sge1) was used as Verticillium inoculation control. Plants were photographed at $14 \mathrm{dpi}$. (B) Fungal biomass was determined by qPCR in Verticillium-inoculated Moneymaker tomato plants at 14 dpi. Bars represent Verticillium ITS levels relative to tomato actin levels (for equilibration) with standard deviation in a sample of three pooled plants. The fungal biomass in tomato plants upon TRV::GUS treatment and subsequent inoculation with the wild-type $V$. dahliae strain JR2 is set to $100 \%$ (control). Asterisks indicate significant differences when compared with the TRV::GUS-treated plants upon inoculation with the $V$. dahliae strain JR2 $(P<0.05)$. The data shown are representative of three independent assays. (C) Relative expression level for the Sge1 gene was determined by using RT-qPCR at 14 days post inoculation with the wild-type V. dahliae strain on TRV::Sge1- and TRV::GUS-treated plants. Bars represent levels of Sge1 transcripts relative to the transcript levels of $V$. dahliae GAPDH (GAPDH, glyceraldehyde-3-phosphate dehydrogenase; for normalization) with standard deviation of a sample of three pooled plants. Sge1 expression in V. dahliae in the TRV::GUS-treated plants upon inoculation the wild-type strain $V$. dahliae is set to 1 . The data shown are representative of three independent experiments. 
treatment and subsequent inoculation with the NLP1 deletion mutant of $V$. dahliae strain JR2 (Figure 3A). Interestingly, upon inoculation with the wild-type V. dahliae strain JR2, a moderate reduction of Verticillium wilt symptoms was observed on Moneymaker tomato plants treated with TRV2::NLP1 when compared to TRV::GUStreated plants (Figure 3A). The plants that were treated with TRV::NLP1 and subsequent inoculation with the wild-type $V$. dahliae strain JR2 showed reduced Verticillium wilt symptoms but were not as diseased as plants upon inoculation with the NLP1 deletion mutant or water (Figure 3A). These data are further supported by fungal biomass quantifications in stem sections of the inoculated plants (Figure 3B). In contrast, no significant Verticillium wilt disease reduction was observed in Moneymaker tomato plants upon the TRV::Sge1 treatment and subsequent inoculation with the wild-type $V$. dahliae strain JR2, although fungal biomass quantifications revealed that less fungal biomass accumulated in planta in the TRV::Sge1-treated plants than the TRV::GUS-treated plants followed by inoculation with the wild-type $V$. dahliae strain JR2 (Figure 4A, B). To determine whether TRV-mediated targeting transcripts of V. dahliae Sge1 in tomato results in complete silencing of the $\mathrm{V}$. dahliae Sge1 gene, we performed reverse transcriptionquantitative polymerase chain reaction (RT-qPCR) to measure relative expression level for the Sge1 gene in V. dahliae JR2 inoculating with the TRV::Sge1-treated plants compared to the TRV::GUS-treated plants. However, only a slight reduction in Sge1 expression in TRV::Sge1-targeted V. dahliae was monitored when compared with TRV::GUS-targeted V. dahliae (Figure 4C). In conclusion, although not all TRVbased RNAi constructs targeting $V$. dahliae transcripts in tomato suppressed Verticillium wilt disease, TRV-mediated transient HIGS against V. dahliae in tomato can be achieved.

\section{HIGS in Ve1-transgenic $A$. thaliana does not impair Ave1-triggerred immunity}

To assess whether HIGS against $V$. dahliae can be made operational in stable transgenic plants by expressing dsRNAs, we exploited hairpin RNA-based RNAi to produce dsRNAs to target $V$. dahliae Ave1 transcripts in Ve1-expressing A. thaliana plants. To this end, a fragment of the $V$. dahliae Ave1 gene was cloned into the 


\section{Chapter 6}

Gateway vector pFAST R03 (Shimada et al., 2010) to obtain the RNAi construct pFAST R03_Ave1 (Figure 1B) that leads to hairpin RNA formation after transcription. A recombinant RNAi construct containing a fragment of the green fluorescent protein (GFP) gene (pFAST R03_GFP) was used as a negative control (Figure 1B). Subsequently, the Ave1 and GFP RNAi constructs were transformed into recipient Ve1-expressing $A$. thaliana plants (Fradin et al., 2011; Figure S2A). No obvious developmental alterations were observed in the transgenic plants when

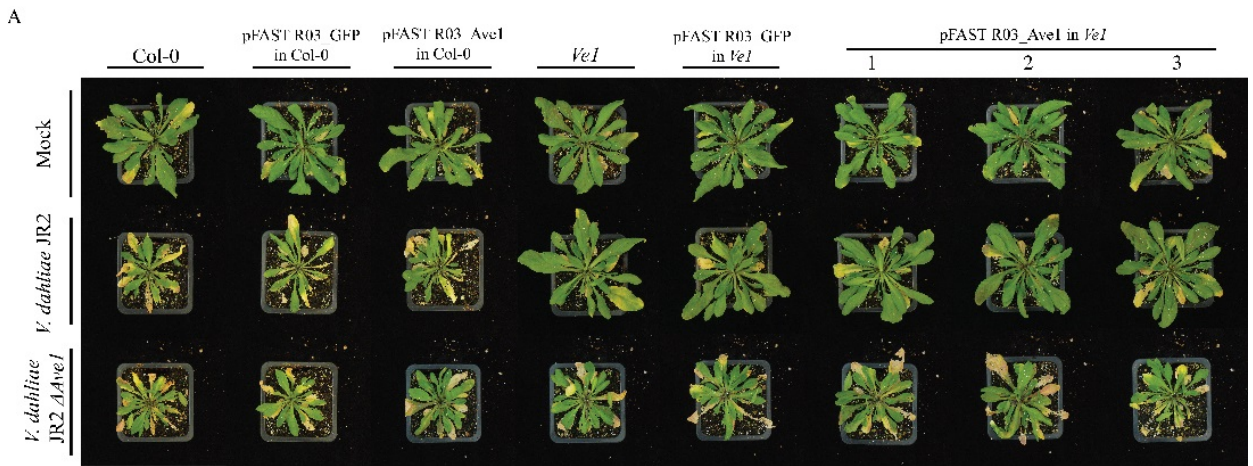

B

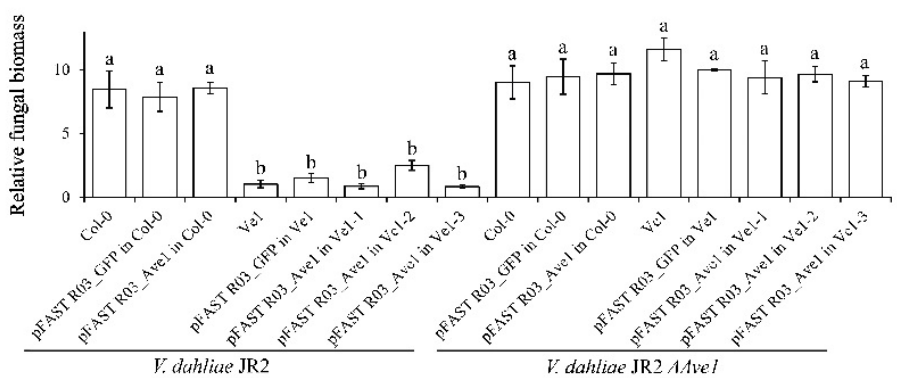

Figure 5. Analysis of Ve1 Arabidopsis thaliana plants expressing RNAi Ave1 construct. (A) Upon inoculation with the race $1 \mathrm{~V}$. dahliae strain JR2, Ve1 plants expressing RNAi Ave1 or GFP construct do not show Verticillium wilt symptoms, whereas typical Verticillium wilt symptoms are recorded on plants with or without tomato Ve1 upon inoculation with either the V. dahliae JR2 or V. dahliae JR2AAve1 at 21 dpi. Col- 0 plants with or without tomato Ve1 were used as a controls. The V. dahliae JR2 $\triangle A v e 1$ strain was used as Verticillium inoculation control. (B) Fungal biomass was determined with qPCR in Verticilliuminoculated Arabidopsis plants at 21 dpi. Bars represent Verticillium ITS levels relative to AtRuBisCo (RuBisCo, ribulose-1, 5-bisphoshate-carboxylase/oxygenase) levels (for equilibration) with standard deviation in a sample of five pooled plants. The fungal biomass in Ve1 plants upon inoculation with the wild-type race $1 \mathrm{~V}$. dahliae strain JR2 is set to 1 (control). Three independent lines carrying the pFAST R03_Ave1 construct are shown (1,2 and 3). Different letter labels indicate significant differences $(P<$ 0.05). The data shown are representative of at least three independent experiments. 
compared with the recipient Col-0 and Ve1 plants (Figure 5A), and three independent Ave1 RNAi lines expressing Ve1 (pFAST R03_Ave1 in Ve1-1, pFAST R03_Ave1 in Ve1-2 and pFAST R03_Ave1 in Ve1-3) as well as transgenic and nontransgenic control lines were inoculated with either the $V$. dahliae race 1 strain JR2 or an Ave1 deletion mutant, and monitored for Verticillium wilt symptoms up to 21 dpi. As expected, upon mock-inoculation or inoculation with the V. dahliae JR2, no disease symptoms were observed in Ve1 plants and GFP-RNAi Ve1 plants (Figure 5A). In contrast, GFP- or Ave1-RNAi Col-0 plants lacking Ve1 were as diseased as non-transformed control lines (Figure 5A). However, despite transcripts for hairpin Ave1 formation in Ave1-RNAi Ve1 plants were detected (Figure S2A), Verticillium wilt symptoms were not observed in Ave1-RNAi Ve1 plants upon inoculation with the wild-type race $1 \mathrm{~V}$. dahliae strain JR2 in repeated assays, while the Ave1 deletion strain caused clear Verticillium wilt symptoms on Ve1 plants (Figure 5A). The phenotypes correlated with the degree of $V$. dahliae colonization as determined with qPCR (Figure 5). These data show that expression of an RNAi construct targeting Ave1 transcripts in A. thaliana plants expressing Ve1 does not compromise Ave1-triggered immunity.

\section{HIGS in A. thaliana can reduce Verticillium wilt}

To further investigate whether HIGS against $V$. dahliae can be established in stable transgenic A. thaliana plants by expressing dsRNAs, V. dahliae NLP1 and Sge1 were targeted. To this end, RNAi constructs pHellsgate 12_NLP1 and pHellsgate 12_Sge1 were generated (Figure 1B). The recombinant RNAi construct carrying a fragment of the GFP gene (pHellsgate 12 _GFP) was used as a negative control (Figure 1B). Subsequently, RNAi constructs targeting NLP1, Sge1, or GFP were transformed into $A$. thaliana ecotype Col-0, and independent NLP1-, Sge1, or GFP-RNAi lines were selected (Figure S2B, C). No phenotypic alterations were observed in NLP1- or Sge1-RNAi plants when compared with the recipient $A$. thaliana Col-0 plants or GFP-RNAi plants (Figure 6A, 7A). Three independent NLP1-RNAi lines (pHellsgate 12_NLP1-1, pHellsgate 12_NLP1-2 and pHellsgate 12_NLP1-3) as well as GFP-RNAi and non-transgenic control lines were assayed for the development of Verticillium wilt symptoms. As expected, markedly compromised Verticillium wilt symptoms were observed on $A$. thaliana plants upon inoculation with the NLP1 deletion 
A

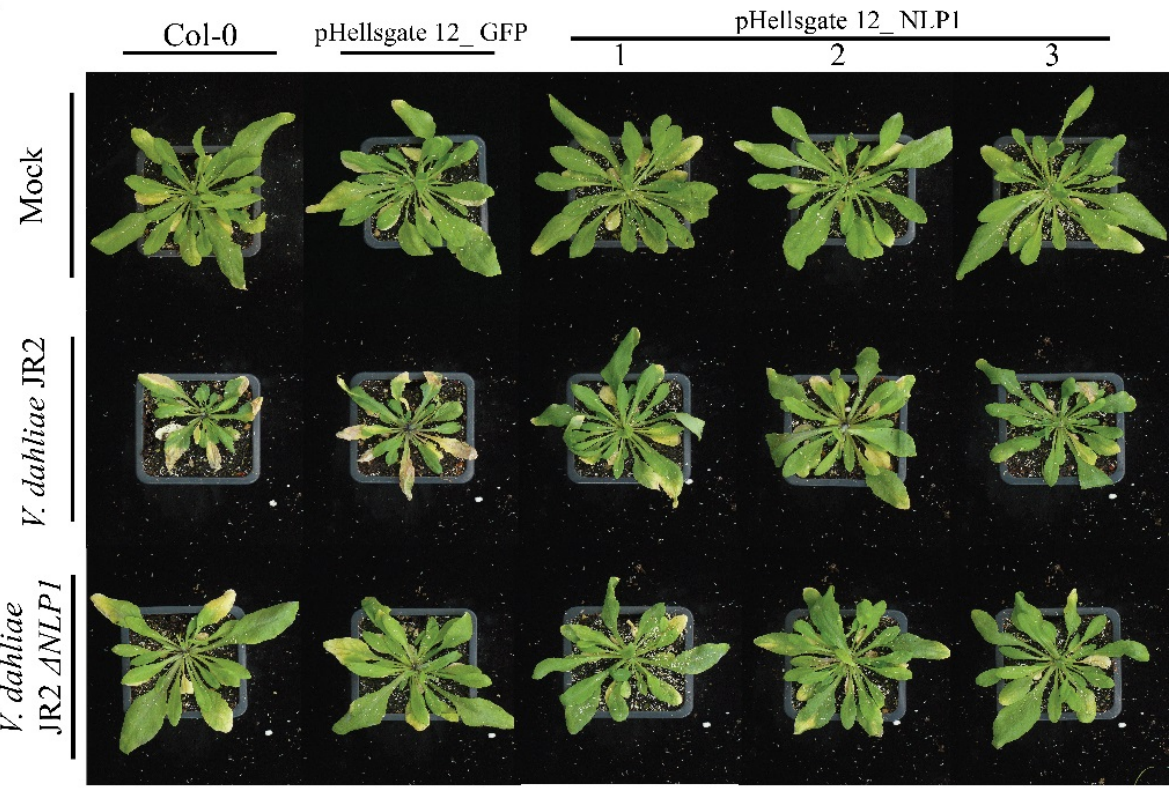

B

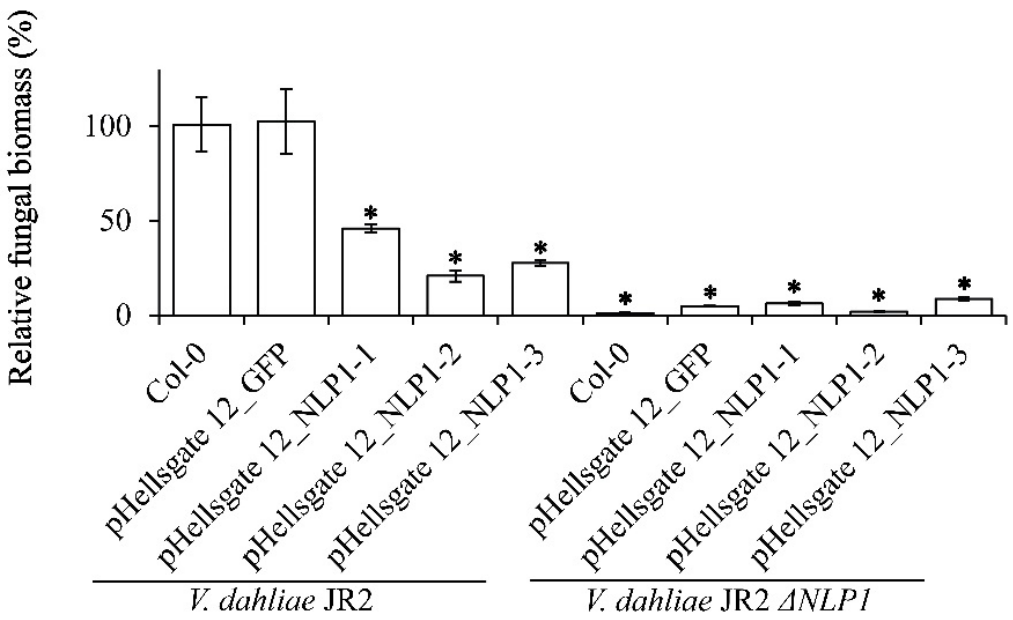

Figure 6. Arabidopsis thaliana Col-0 plants expressing NLP1 RNAi construct show enhanced resistance against Verticillium dahliae. (A) Typical appearance of non-transgenic A. thaliana and transgenic lines carrying the pHellsgate 12_NLP1 construct to target NLP1 transcripts upon mockinoculation or inoculation with V. dahliae strain JR2 or V. dahliae JR2 $\triangle N L P 1$ at 21 dpi. (B) Fungal biomass was determined with qPCR in Verticillium-inoculated Arabidopsis plants at $21 \mathrm{dpi}$. Bars represent Verticillium ITS levels relative to AtRuBisCo (RuBisCo, ribulose-1, 5-bisphoshate-carboxylase/oxygenase) levels (for equilibration) with standard deviation in a sample of five pooled plants. The fungal biomass in Col-0 is set to $100 \%$ (control). Three independent lines carrying the pHellsgate 12_NLP1 construct are shown (1, 2 and 3). Asterisks indicate significant differences when compared with Col-0 $(P<0.05)$. The data shown are representative of three independent experiments. 
mutant (Figure 6A). Interestingly, upon inoculation with the $V$. dahliae JR2, a significant reduction of Verticillium wilt symptoms was observed in NLP1-RNAi plants when compared with GFP-RNAi and non-transgenic controls (Figure 6A). These data are further supported by fungal biomass quantifications in stem sections of the inoculated plants (Figure 6B). Additionally, three independent Sge1RNAi lines (pHellsgate 12_Sge1-1, pHellsgate 12_Sge1-2 and pHellsgate 12_Sge1-3) as well as GFP-RNAi and non-transformed control lines were assayed for the development of Verticillium wilt disease. Intriguingly, we observed a marked reduction of Verticillium wilt symptoms in Sge1-RNAi A. thaliana lines inoculated with $V$. dahliae JR2 (Figure 7A). In contrast, GFP-RNAi $A$. thaliana lines were as susceptible as non-transgenic control lines (Figure 7A). The phenotypes correlated with the level of $V$. dahliae biomass as determined with qPCR (Figure 7). Collectively, these results suggest that, although not all RNAi constructs targeting $V$. dahliae transcripts in $A$. thaliana induced HIGS against $V$. dahliae, hairpin RNAmediated HIGS in $A$. thaliana can reduce Verticillium wilt disease.

\section{DISCUSSION}

In this manuscript, we show that HIGS against $V$. dahliae can be achieved through TRV-based fungal gene silencing in tomato, and through hairpin RNA-mediated fungal gene silencing in stable transgenic $A$. thaliana lines. We established the TRVmediated HIGS assay through targeting $V$. dahliae Ave1 transcripts in Ve1 tomato plants, and further used this approach to assess whether HIGS against $V$. dahliae in tomato can be established through TRV constructs targeting previously identified $V$. dahliae virulence factors. We also investigated whether HIGS against $V$. dahliae can be established in transgenic $A$. thaliana plants through hairpin RNA-based RNAi constructs targeting transcripts of the same previously identified $V$. dahliae virulence genes. Our results clearly show that plants transiently (in tomato) or stably (in A. thaliana) expressing RNAi constructs targeting transcripts of genes that are essential for $V$. dahliae pathogenicity can become protected from Verticillium wilt disease. Our results are in line with, and extend beyond, recent reports on protection of cotton plants stably expressing an RNAi construct against $V$. dahliae (Zhang et al., 2016), and on bidirectional cross-kingdom RNAi and fungal uptake of external RNAs to confer plant protection (Wang et al., 2016). 


\section{Chapter 6}

A

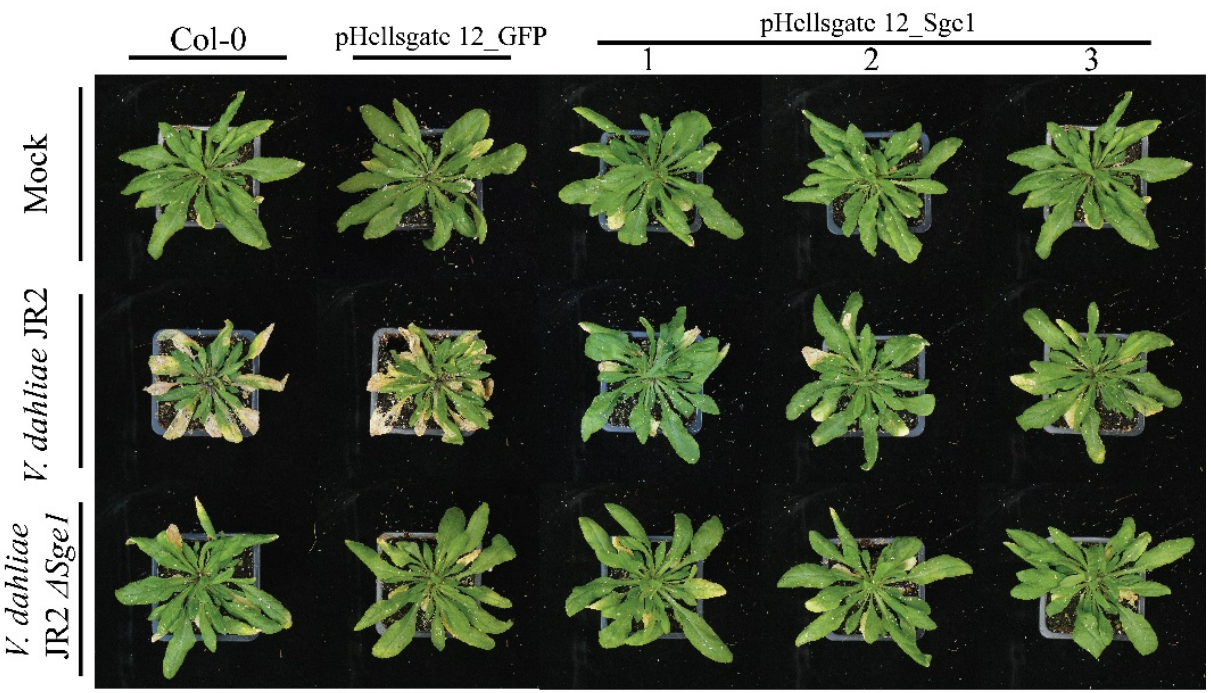

B

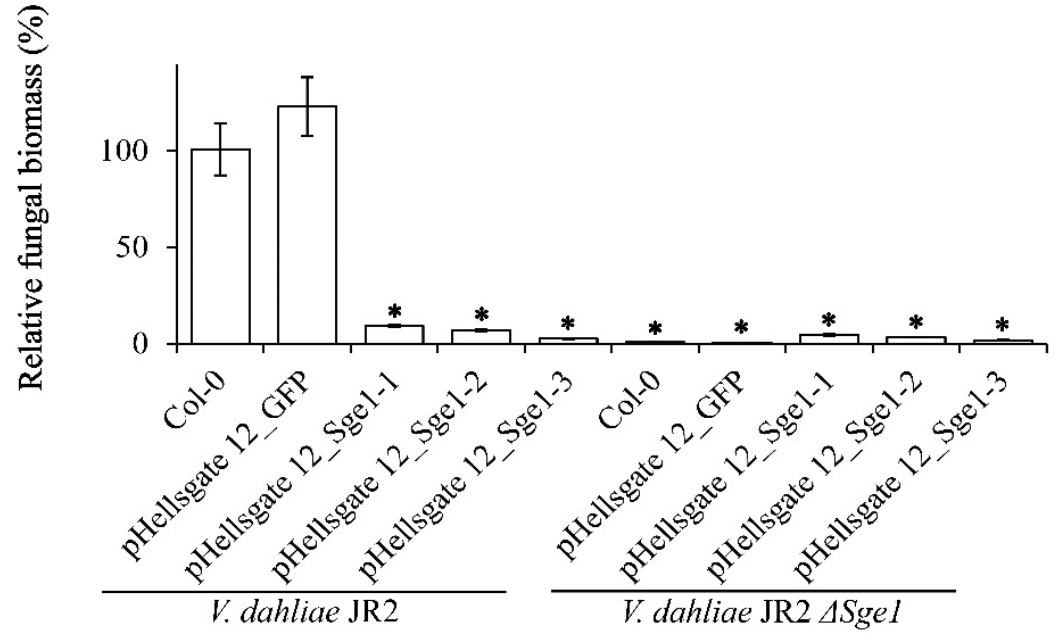

Figure 7. Arabidopsis thaliana Col-0 plants expressing Sge1 RNAi construct show enhanced resistance against Verticillium dahliae. (A) Typical appearance of non-transgenic A. thaliana and transgenic lines harboring the pHellsgate 12_Sge1 construct to target Sge1 transcripts upon mockinoculation or inoculation with V. dahliae strain JR2 or V. dahliae JR2 $\Delta$ Sge 1 at 21 dpi. (B) Fungal biomass was determined with qPCR in Verticillium-inoculated Arabidopsis plants at $21 \mathrm{dpi}$. Bars represent Verticillium ITS levels relative to AtRuBisCo (RuBisCo, ribulose-1, 5-bisphoshate-carboxylase/oxygenase) levels (for equilibration) with standard deviation in a sample of five pooled plants. The fungal biomass in Col-0 is set to $100 \%$ (control). Three independent lines carrying the pHellsgate 12 Sge1 construct are shown $(1,2$ and 3). Asterisks indicate significant differences when compared with Col-0 $(P<0.05)$. The data shown are representative of three independent experiments. 
Reports on HIGS against fungal pathogen infections have accumulated over recent years (Andrade et al., 2015; Chen et al., 2016; Cheng et al., 2015; Ghag et al., 2014; Hu et al., 2015; Koch et al., 2013; Panwar et al., 2013; Yin et al., 2011; 2015; Zhang et al., 2012; Zhou et al., 2016). Among these reports, disease suppression was observed upon silencing of various types of genes, including those that encode the biosynthesis of structural components such chitin and ergosterol, but also genes involved in developmental regulation, secondary metabolism and pathogenicity. Therefore, the selection of suitable target genes is arguably the most important prerequisite for developing a successful HIGS against fungal pathogens. We selected HIGS target genes based on our previous studies of gene deletion mutants in $V$. dahliae with significantly compromised virulence (NLP1, Santhanam et al., 2013; Sge1, Santhanam and Thomma, 2013). Beforehand, it was not clear whether silencing of such genes rather than gene deletion would lead to visible virulence phenotypes, as the protein encoded by the target gene may not be completely absent. Indeed, TRV-mediated HIGS of Sge1 in tomato did not lead to compromised Verticillium wilt symptoms, which may be explained by the incomplete silencing of the Sge1 gene in V. dahliae (Figure 4C). This also explains why RNAi-mediated HIGS of $V$. dahliae Ave1 did not compromise Ve1-mediated immunity in transgenic $A$. thaliana plants. Also in the cases where the expected visual phenotypes were obtained, fungal biomass quantifications revealed that more fungal biomass accumulated in the inoculated plants upon HIGS of the fungal target gene than upon inoculation with the corresponding $V$. dahliae deletion mutant (Figure 3B, 4B, 6B, 7B). Thus, hypothetically, RNAi may not be appropriate to target genes of which the activity is required early in the infection process when RNAi may not have taken its full effect, or genes of which a low-dose of transcripts is biologically active.

Mobility of small RNAs within organisms is a well-known phenomenon, facilitating gene silencing in adjacent cells and surrounding or even distant tissues (Weiberg et al., 2015). Over recent years, several examples of exchange of small RNAs between host plants and invading pathogens have been described, although the mechanistic details of the actual exchange remains to be elucidated (Knip et al., 2014). Nevertheless, small RNA-based bidirectional cross-kingdom gene silencing has been proposed as a common mechanism for cross-kingdom gene regulation in plant-pathogen interactions (Chaloner et al., 2016; Wang et al., 2016; Weiberg et al., 
2015). For example, endogenous small RNAs from the fungus Botrytis cinerea have been proposed to transfer into host plants to target defense-related plant transcripts to promote disease development (Weiberg et al., 2013). In this manner, HIGS taps into a process that naturally occurs between plants and pathogens. A search for pathogen-derived small RNAs matching transcripts of host plants or plant-derived small RNAs targeting transcripts of the invading pathogens may facilitate the development of HIGS strategies to engineer resistance in plants against pathogens for which no natural resistance sources have been identified.

\section{MATERIALS AND METHODS}

\section{Plant growth conditions and manipulations}

Plants were grown in commercial potting soil (Horticoop, Bleiswijk, the Netherlands) at $21^{\circ} \mathrm{C} / 19^{\circ} \mathrm{C}$ during $16 \mathrm{~h} / 8 \mathrm{~h}$ light/dark photoperiods, respectively, in the climate chamber or the greenhouse (Unifarm, Wageningen, the Netherlands) with a relative humidity of $\sim 75 \%$, and $100 \mathrm{~W} \cdot \mathrm{m}^{-2}$ supplemental light when light intensity dropped below $150 \mathrm{~W} \cdot \mathrm{m}^{-2}$. A. thaliana transformations were performed as described (Clough and Bent, 1998).

\section{Generation of the constructs}

The Gateway-compatible Tobacco rattle virus (TRV) two-component Agrobacteriummediated expression system was used for gene silencing in tomato as previously described (Liu et al., 2002), while the Gateway-compatible vectors pFAST R03 (Shimada et al., 2010) and pHellsgate 12 (Helliwell and Waterhouse, 2003) for hairpin RNA-mediated gene silencing were used to generate stable A thaliana transformants. The three V. dahliae genes Ave1 (de Jonge et al., 2012), NLP1 (Santhanam et al., 2013) and Sge1 (Santhanam and Thomma, 2013) were selected for RNAi-based HIGS. Gene annotations for V. dahliae Ave1, Sge1 and NLP1 were obtained from Ensembl Genomes database (http://fungi.ensembl.org/Verticillium dahliaejr2/Info/Index). Selected DNA fragments were amplified by PCR from the corresponding plasmids using gene-specific primers listed in Table S1. The DNA fragments were cloned into pDONR207 by using the Gateway ${ }^{\circledR}$ BP Clonase ${ }^{\circledR}$ II Enzyme Mix (Invitrogen, Carlsbad, USA) to generate entry vectors, and all the entry vectors were verified by DNA sequencing (Eurofins Genomics, Ebersberg, 
Germany). Subsequently, the entry vector pDONR207 carrying the Ave1 fragment was transferred to TRV2 and pFAST R03 to generate constructs TRV2::Ave1 and pFAST R03_Ave1 (Figure 1), while pDONR207 entry vectors carrying NLP1 or Sge1 fragment were recombined into pTRV2 and pHellsgate 12 to generate constructs TRV2::NLP1, TRV2::Sge1, pHellsgate 12_NLP1 and pHellsgate 12_Sge1 (Figure 1) by using Gateway ${ }^{\circledR}$ LR Clonase ${ }^{\circledR}$ II Enzyme Mix (Invitrogen, Carlsbad, USA). All constructs were transformed to Agrobacterium tumefaciens strain GV3101 (pMP90) by electroporation.

\section{TRV treatment}

TRV vectors were agroinfiltrated as previously described (Liu et al., 2002; Fradin et al., 2011). Briefly, cotyledons of 10-day-old tomato (Solanum lycopersicum cv. Moneymaker (ve1) or 35S::Ve1 tomato (Ve1); Fradin et al., 2009) were infiltrated as 1:1 mixtures of pTRV1 and pTRV2 constructs. 10-15 days after TRV inoculation, plants were inoculated with race $1 \mathrm{~V}$. dahliae strain JR2 (Faino et al., 2015); the corresponding mutants: $V$. dahliae JR2 $\Delta$ Ave1 (de Jonge et al., 2012); V. dahliae JR2 $\triangle N L P 1$ (Santhanam et al., 2013); V. dahliae JR2 2 Sge1 (Santhanam and Thomma, 2013); or tap water as control. The inoculated plants were evaluated by observing disease symptoms up to 14 days post inoculation (dpi).

\section{Verticillium wilt disease and fungal recovery assays}

$V$. dahliae was grown on potato dextrose agar (PDA; Oxoid, Basingstoke, UK) at $22^{\circ} \mathrm{C}$, and conidia were collected from 7- to 10-day-old PDA plates and washed with tap water. Disease assays on tomato plants were performed as previously described (Fradin et al., 2009). Briefly, twenty-day-old Ve1 tomato plants (for Verticillium inoculation after TRV treatment) or ten-day-old Ve1 and ve1 tomato plants (for inoculation with Verticillium colonies re-isolated from infected tomato plants) were uprooted, the roots were rinsed in water, dipped for $5 \mathrm{~min}$ in a suspension of $10^{6}$ conidiospores/mL water, and transplanted to commercial potting soil (Horticoop, Bleiswijk, the Netherlands). Verticillium outgrowth assays of Ve1 tomato plants, canopy area measurement and fungal biomass quantification in tomato plants were performed as previously described (Fradin et al., 2009; Santhanam et al., 2013). Verticillium disease assay on A. thaliana, as well as fungal biomass quantification in 


\section{Chapter 6}

infected $A$. thaliana plants were performed as previously described (Ellendorff et al., 2009; Song et al., 2016). The fungus-specific primer ITS1-F, based on the internal transcribed spacer (ITS) region of the ribosomal DNA, in combination with the $V$. dahliae-specific reverse primer ST-Ve1-R (Ellendorff et al., 2009) were used to measure fungal colonization. Primers for tomato actin and A. thaliana RuBisCo (Table S1) were used as endogenous plant control as described earlier (Santhanam et al., 2013; Song et al., 2016).

\section{Quantitative PCR (qPCR) and Reverse transcription-qPCR (RT-qPCR)}

To determine expression of $V$. dahliae Sge1 gene for silencing, stems of TRV-treated tomato plants were harvested at 14 days post Verticillium inoculation as described above, and flash frozen in liquid nitrogen, and stored at $-80{ }^{\circ} \mathrm{C}$ for total RNA isolation.

To check Ave1, NLP1, Sge1, GFP DNA fragment presence in the transcripts of the corresponding transgenic $A$. thaliana lines, two-week-old transgenic and nontransgenic $A$. thaliana lines were harvested and ground into a powder in liquid nitrogen. Total RNA extraction, cDNA synthesis and RT-PCR were performed as described earlier (Song et al., 2016). Primers for hairpin expression analysis are listed in Table S1. To analyze expression of Sge1 gene for silencing, RT-qPCR was conducted by using primers Sge1-F(qRT) and Sge1-R(qRT) with V. dahliae GAPDH as an endogenous control (Table S1), employing an ABI 7300 PCR system (Applied Biosystems, Foster City, USA) with the qPCR Core kit for SYBR Green I (Eurogentec Nederland BV, Maastricht, the Netherlands) as previously described (Santhanam and Thomma, 2013).

\section{ACKNOWLEDGEMENTS}

Y.S. acknowledges a PhD fellowship from the China Scholarship Council (CSC). B.P.H.J.T. is supported by a Vici grant of the Research Council for Earth and Life sciences (ALW) of the Netherlands Organization for Scientific Research (NWO). Constantinos Patinios and Yidong Wang are acknowledged for technical assistance and Bert Essenstam for excellent plant care at Unifarm. The authors declare no conflict of interest. 


\section{REFERENCES}

Andrade C, Tinoco M, Rieth A, Maia F, Aragão F (2015) Host-induced gene silencing in the necrotrophic fungal pathogen Sclerotinia sclerotiorum. Plant Pathol 65: 626-632.

Antanaviciute L, Šurbanovski N, Harrison N, McLeary K, Simpson D, Wilson F, Sargent D, Harrison R (2015) Mapping QTL associated with Verticillium dahliae resistance in the cultivated strawberry (Fragaria $\times$ ananassa). Hortic Res 2: 15009.

Barrow JR (1970) Heterozygosity in inheritance of Verticillium wilt tolerance in cotton. Phytopathology 60: 301-303.

Baulcombe D (2005) RNA silencing. Trends Biochem Sci 30: 290-293.

Baum JA, Bogaert T, Clinton W, Heck GR, Feldmann P, Ilagan O, Johnson S, Plaetinck G, Munyikwa T, Pleau M (2007) Control of coleopteran insect pests through RNA interference. Nat Biotechnol 25: 1322-1326.

Bolek Y, El-Zik KM, Pepper AE, Bell AA, Magill CW, Thaxton PM, Reddy OUK (2005) Mapping of verticillium wilt resistance genes in cotton. Plant Sci 168: 1581-1590.

Chaloner T, van Kan JA, Grant-Downton RT (2016) RNA 'Information Warfare'in pathogenic and mutualistic interactions. Trends Plant Sci 21: 738-748.

Chen W, Kastner C, Nowara D, Oliveira-Garcia E, Rutten T, Zhao Y, Deising HB, Kumlehn J, Schweizer P (2016) Host-induced silencing of Fusarium culmorumgenes protects wheat from infection. J Exp Bot 67: 4979-4991.

Cheng W, Song XS, Li HP, Cao LH, Sun K, Qiu XL, Xu YB, Yang P, Huang T, Zhang JB (2015) Hostinduced gene silencing of an essential chitin synthase gene confers durable resistance to Fusarium head blight and seedling blight in wheat. Plant Biotechnol J 13: 1335-1345.

Christopoulou M, Wo SR-C, Kozik A, McHale LK, Truco M-J, Wroblewski T, Michelmore R (2015) Genome-wide architecture of disease resistance genes in lettuce. G3 5: 2655-2669.

Clough SJ, Bent AF (1998) Floral dip: a simplified method for Agrobacterium-mediated transformation of Arabidopsis thaliana. Plant J 16: 735-743.

de Jonge R, van Esse HP, Maruthachalam K, Bolton MD, Santhanam P, Saber MK, Zhang Z, Usami T, Lievens B, Subbarao KV, Thomma BPHJ (2012) Tomato immune receptor Ve1 recognizes effector of multiple fungal pathogens uncovered by genome and RNA sequencing. Proc Natl Acad Sci USA 109: 5110-5115.

Dobinson K, Tenuta G, Lazarovits G (1996) Occurrence of race 2 of Verticillium dahliae in processing tomato fields in southwestern Ontario. Can J Plant Pathol 18: 55-58.

Duan C-G, Wang C-H, Guo H-S (2012) Application of RNA silencing to plant disease resistance. Silence 3: 5 .

Ellendorff U, Fradin EF, De Jonge R, Thomma BPHJ (2009) RNA silencing is required for Arabidopsis defence against Verticillium wilt disease. J Exp Bot 60: 591-602.

Escobar MA, Civerolo EL, Summerfelt KR, Dandekar AM (2001) RNAi-mediated oncogene silencing confers resistance to crown gall tumorigenesis. Proc Natl Acad Sci USA 98: 13437-13442.

Escobar MA, Leslie CA, McGranahan GH, Dandekar AM (2002) Silencing crown gall disease in walnut (Juglans regia L.). Plant Sci 163: 591-597.

Faino L, Seidl MF, Datema E, van den Berg GC, Janssen A, Wittenberg AH, Thomma BPHJ (2015) Single-molecule real-time sequencing combined with optical mapping yields completely finished fungal genome. mBio 6: e00936-00915.

Fradin EF, Abd-El-Haliem A, Masini L, van den Berg GC, Joosten MH, Thomma BPHJ (2011) Interfamily transfer of tomato Ve1 mediates Verticillium resistance in Arabidopsis. Plant Physiol 


\section{Chapter 6}

156: $2255-2265$.

Fradin EF, Thomma BPHJ (2006) Physiology and molecular aspects of Verticillium wilt diseases caused by V. dahliae and V. albo-atrum. Mol Plant Pathol 7: 71-86.

Fradin EF, Zhang Z, Ayala JCJ, Castroverde CD, Nazar RN, Robb J, Liu C-M, Thomma BPHJ (2009) Genetic dissection of Verticillium wilt resistance mediated by tomato Ve1. Plant Physiol 150: 320332.

Fradin EF, Zhang Z, Rovenich H, Song Y, Liebrand TW, Masini L, van den Berg GC, Joosten MH, Thomma BPHJ (2014) Functional analysis of the tomato immune receptor Ve1 through domain swaps with its non-functional homolog Ve2 PLOS ONE, 9: e88208.

Ghag SB, Shekhawat UK, Ganapathi TR (2014) Host-induced post-transcriptional hairpin RNAmediated gene silencing of vital fungal genes confers efficient resistance against Fusarium wilt in banana. Plant Biotechnol J 12: 541-553.

Govindarajulu M, Epstein L, Wroblewski T, Michelmore RW (2015) Host-induced gene silencing inhibits the biotrophic pathogen causing downy mildew of lettuce. Plant Biotechnol J 13: 875-883.

Hayes RJ, McHale LK, Vallad GE, Truco MJ, Michelmore RW, Klosterman SJ, Maruthachalam K, Subbarao KV (2011) The inheritance of resistance to Verticillium wilt caused by race 1 isolates of Verticillium dahliae in the lettuce cultivar La Brillante. Theor Appl Genet 123: 509-517.

Helliwell C, Waterhouse P (2003) Constructs and methods for high-throughput gene silencing in plants. Methods 30: 289-295.

Hu Z, Parekh U, Maruta N, Trusov Y, Botella JR (2015) Down-regulation of Fusarium oxysporum endogenous genes by host-delivered RNA interference enhances disease resistance. Front Chem 3: 1.

Huang G, Allen R, Davis EL, Baum TJ, Hussey RS (2006) Engineering broad root-knot resistance in transgenic plants by RNAi silencing of a conserved and essential root-knot nematode parasitism gene. Proc Natl Acad Sci USA 103: 14302-14306.

Inderbitzin P, Bostock RM, Davis RM, Usami T, Platt HW, Subbarao KV (2011) Phylogenetics and taxonomy of the fungal vascular wilt pathogen Verticillium, with the descriptions of five new species. PLOS ONE 6: e28341.

Jahan SN, Åsman AK, Corcoran P, Fogelqvist J, Vetukuri RR, Dixelius C (2015) Plant-mediated gene silencing restricts growth of the potato late blight pathogen Phytophthora infestans. J Exp Bot 66: 2785-2794.

Jakse J, Cerenak A, Radisek S, Satovic Z, Luthar Z, Javornik B (2013) Identification of quantitative trait loci for resistance to Verticillium wilt and yield parameters in hop (Humulus lupulus L.). Theor Appl Genet 126: 1431-1443.

Kawchuk LM, Hachey J, Lynch DR, Kulcsar F, van Rooijen G, Waterer DR, Robertson A, Kokko E, Byers R, Howard RJ (2001) Tomato Ve disease resistance genes encode cell surface-like receptors. Proc Natl Acad Sci USA 98: 6511-6515.

Klimes A, Dobinson KF, Klosterman SJ, Thomma BPHJ (2015) Genomics spurs rapid advances in our understanding of the basic biology of vascular wilt pathogens in the genus Verticillium. Annu Rev Phytopathol 53: 181-198.

Klosterman SJ, Atallah ZK, Vallad GE, Subbarao KV (2009) Diversity, pathogenicity, and management of Verticillium species. Ann Rew Phytopathol 47: 39-62.

Knip M, Constantin ME, Thordal-Christensen H (2014) Trans-kingdom cross-talk: small RNAs on the move. PLoS Genet 10: e1004602.

Koch A, Kumar N, Weber L, Keller H, Imani J, Kogel K-H (2013) Host-induced gene silencing of cytochrome P450 lanosterol C14 $\alpha$-demethylase-encoding genes confers strong resistance to 
Fusarium species. Proc Natl Acad Sci USA 110: 19324-19329.

Liu Y, Schiff M, Dinesh-Kumar S (2002) Virus-induced gene silencing in tomato. Plant J 31: 777-786.

Lynch D, Kawchuk L, Hachey J, Bains P, Howard R (1997) Identification of a gene conferring high levels of resistance to Verticillium wilt in Solanum chacoense. Plant Dis 81: 1011-1014.

Mao Y-B, Cai W-J, Wang J-W, Hong G-J, Tao X-Y, Wang L-J, Huang Y-P, Chen X-Y (2007) Silencing a cotton bollworm P450 monooxygenase gene by plant-mediated RNAi impairs larval tolerance of gossypol. Nat Biotechnol 25: 1307-1313.

Mascia T, Nigro F, Abdallah A, Ferrara M, De Stradis A, Faedda R, Palukaitis P, Gallitelli D (2014) Gene silencing and gene expression in phytopathogenic fungi using a plant virus vector. Proc Natl Acad Sci USA 111: 4291-4296.

Mert M, Kurt S, Gencer O, Akiscan Y, Boyaci K, Tok F (2005) Inheritance of resistance to Verticillium wilt (Verticillium dahliae) in cotton (Gossypium hirsutum L.). Plant Breed 124: 102-104.

Nowara D, Gay A, Lacomme C, Shaw J, Ridout C, Douchkov D, Hensel G, Kumlehn J, Schweizer P (2010) HIGS: host-induced gene silencing in the obligate biotrophic fungal pathogen Blumeria graminis. Plant Cell 22: 3130-3141.

Panwar V, McCallum B, Bakkeren G (2013) Host-induced gene silencing of wheat leaf rust fungus Puccinia triticina pathogenicity genes mediated by the Barley stripe mosaic virus. Plant Mol Biol 81: 595-608.

Pegg GF (1974) Verticillium diseases. Rev Plant Pathol 53: 157-182.

Pegg GF, Brady BL (2002) Verticillium wilts. Wallingord, Oxfordhire: CABI Publishing.

Putt ED (1964) Breeding behaviour of resistance to leaf mottle or Verticillium in sunflowers. Crop Sci 4: 177-179.

Ruiz-Ferrer V, Voinnet 0 (2009) Roles of plant small RNAs in biotic stress responses. Annu Rev Plant Biol 60: 485-510.

Sanju S, Siddappa S, Thakur A, Shukla PK, Srivastava N, Pattanayak D, Sharma S, Singh B (2015) Host-mediated gene silencing of a single effector gene from the potato pathogen Phytophthora infestans imparts partial resistance to late blight disease. Funct Integr Genomics 15: 697-706.

Santhanam P, Thomma BPHJ (2013) Verticillium dahliae Sge1 differentially regulates expression of candidate effector genes. Mol Plant-Microbe Interact 26: 249-256.

Santhanam P, van Esse HP, Albert I, Faino L, Nürnberger T, Thomma BPHJ (2013) Evidence for functional diversification within a fungal NEP1-like protein family. Mol Plant-Microbe Interact 26: 278-286.

Schaible L, Cannon OS, Waddoups V (1951) Inheritance of resistance to Verticillium wilt in a tomato cross. Phytopathology 41: 986-990.

Senthil-Kumar M, Hema R, Anand A, Kang L, Udayakumar M, Mysore KS (2007) A systematic study to determine the extent of gene silencing in Nicotiana benthamiana and other Solanaceae species when heterologous gene sequences are used for virus-induced gene silencing. New Phytol 176: 782-791.

Shimada TL, Shimada T, Hara-Nishimura I (2010) A rapid and non-destructive screenable marker, FAST, for identifying transformed seeds of Arabidopsis thaliana. Plant J 61: 519-528.

Simko I, Costanzo S, Haynes K, Christ B, Jones R (2004) Linkage disequilibrium mapping of a Verticillium dahliae resistance quantitative trait locus in tetraploid potato (Solanum tuberosum) through a candidate gene approach. Theor Appl Genet 108: 217-224.

Song Y, Zhang Z, Seidl MF, Majer A, Jakse J, Javornik B, Thomma BPHJ (2017) Broad taxonomic characterization of Verticillium wilt resistance genes reveals ancient origin of the tomato Ve1 immune receptor. Mol Plant Pathol 18: 195-209. 


\section{Chapter 6}

Thomma BPHJ, Nürnberger T, Joosten MH (2011) Of PAMPs and effectors: the blurred PTI-ETI dichotomy. Plant Cell 23: 4-15.

Tinoco ML, Dias BB, Dall'Astta RC, Pamphile JA, Aragão FJ (2010) In vivo trans-specific gene silencing in fungal cells by in planta expression of a double-stranded RNA. BMC Biol 8: 27.

Tomilov AA, Tomilova NB, Wroblewski T, Michelmore R, Yoder JI (2008) Trans-specific gene silencing between host and parasitic plants. Plant J 56: 389-397.

Wang G, Fiers M, Ellendorff U, Wang Z, de Wit PJ, Angenent GC, Thomma BPHJ (2010) The diverse roles of extracellular leucine-rich repeat-containing receptor-like proteins in plants. Crit Rev Plant Sci 29: 285-299.

Wang HM, Lin ZX, Zhang XL, Chen W, Guo XP, Nie YC, Li YH (2008) Mapping and quantitative trait loci analysis of verticillium wilt resistance genes in cotton. J Integr Plant Biol 50: 174-182.

Wang M, Weiberg A, Lin FM, Thomma BPHJ, Huang HD, Jin HL (2016). Bidirectional cross-kingdom RNAi and fungal uptake of external RNAs confer plant protection. Nat Plants 2: 16151.

Weiberg A, Bellinger M, Jin H (2015) Conversations between kingdoms: small RNAs. Curr Opin Biotechnol 32: 207-215.

Weiberg A, Wang M, Lin F-M, Zhao H, Zhang Z, Kaloshian I, Huang H-D, Jin H (2013) Fungal small RNAs suppress plant immunity by hijacking host RNA interference pathways. Science 342: 118123.

Yang C, Guo W, Li G, Gao F, Lin S, Zhang T (2008) QTLs mapping for Verticillium wilt resistance at seedling and maturity stages in Gossypium barbadense L. Plant Sci 174: 290-298.

Yin CT, Downey SI, Klages-Mundt NL, Ramachandran S, Chen X, Szabo LJ, Pumphrey M, Hulbert SH (2015) Identification of promising host-induced silencing targets among genes preferentially transcribed in haustoria of Puccinia. BMC Genomics 16: 579.

Yin CT, Jurgenson JE, Hulbert SH (2011) Development of a host-induced RNAi system in the wheat stripe rust fungus Puccinia striiformis f. sp. tritici. Mol Plant-Microbe Interact24: 554-561.

Zhang H, Guo J, Voegele RT, Zhang J, Duan Y, Luo H, Kang Z (2012) Functional characterization of calcineurin homologs PSCNA1/PSCNB1 in Puccinia striiformis f. sp. tritici using a host-induced RNAi system. PLOS ONE 7: e49262.

Zhang T, Jin Y, Zhao J-H, Gao F, Zhou B-J, Fang Y-Y, Guo H-S (2016) Host-induced gene silencing of the target gene in fungal cells confers effective resistance to the cotton wilt disease pathogen Verticillium dahliae. Mol Plant 9: 939-942.

Zhang Z, Song Y, Liu C-M, Thomma BPHJ (2014) Mutational analysis of the Ve1 immune receptor that mediates Verticillium resistance in tomato. PLOS ONE, 9: e99511.

Zhou B, Bailey A, Niblett C, Qu R (2016) Control of brown patch (Rhizoctonia solani) in tall fescue (Festuca arundinacea Schreb.) by host induced gene silencing. Plant Cell Rep 35: 791-802. 


\section{SUPPLEMENTAL INFORMATION}

A

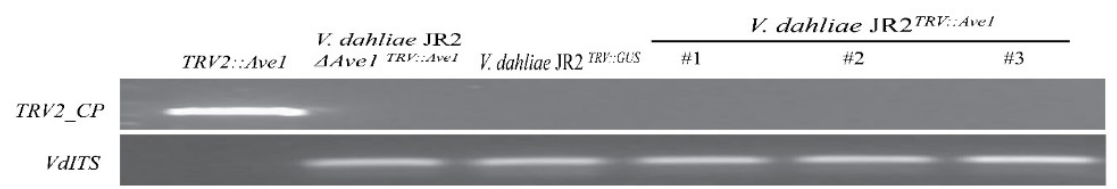

B
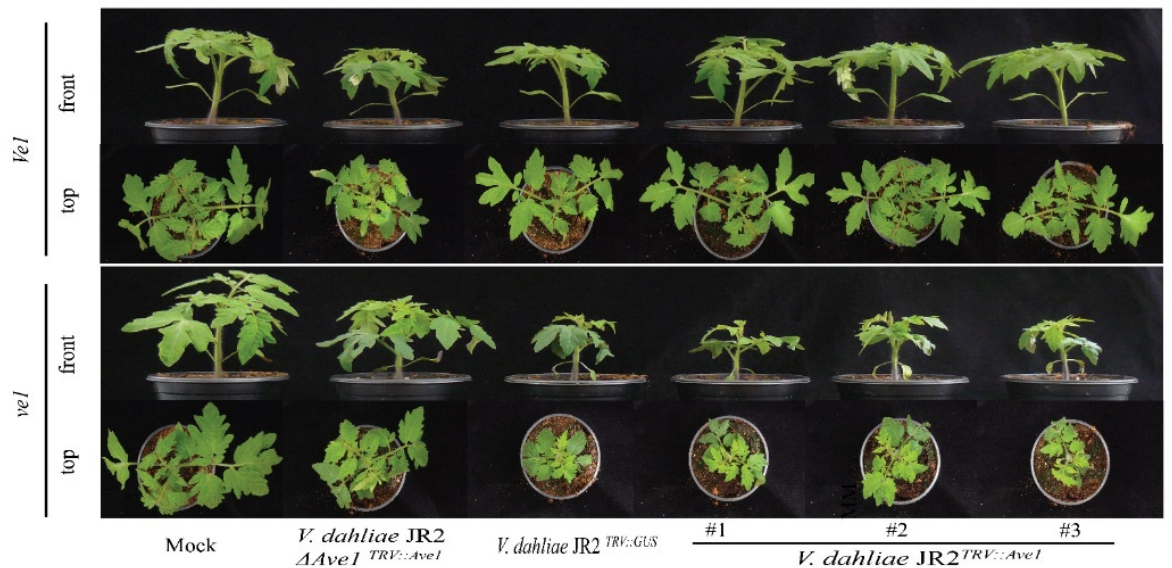

C

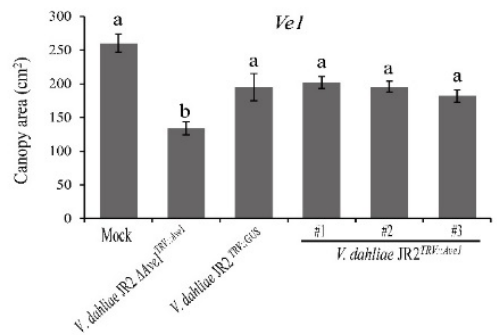

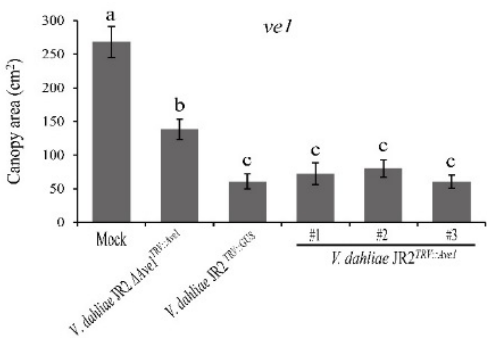

Figure S1. Analysis of Verticillium dahliae colonies re-isolated from TRV-treated Ve1 tomato plants. (A) Viral coat protein gene fragment $\left(T R V 2_{-} C P\right)$ was not detected by PCR in re-isolated V. dahliae colonies V. dahliae JR2 $\triangle A v e 1^{\text {TRV:Ave1, }}$. dahliae JR2 TRV:GUS and V. dahliae JR2 ${ }^{\text {TRV:Ave1 }}$ (\#1, \#2 and \#3) that grew from wild-type $V$. dahliae-inoculated TRV::GUS-treated plants, Ave1 deletion strain-inoculated TRV::Ave1-treated plants and wild-type $V$. dahliae-inoculated TRV::Ave1-treated plants, respectively. Construct TRV::Ave1 was used as a positive control. As an endogenous control, a fragment of the $V$. dahliae ITS was amplified from all re-isolated V. dahliae colonies. (B) Typical appearance of Ve1 tomato plants (Ve1) and Moneymaker tomato plants lacking Ve1 (ve1) upon mock-inoculation or inoculation

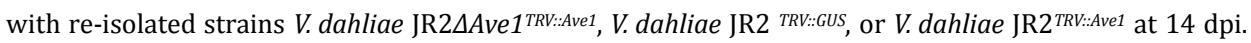
(C) Average canopy area of $10 \mathrm{Ve} 1$ plants inoculated with re-isolated strains described above or mock inoculation. (D) Average canopy area of 10 ve1 plants inoculated with re-isolated strains described above or mock inoculation. Different letters indicate significant differences $(P<0.05)$. The data shown are representative of three independent assays. 


\section{Chapter 6}

A

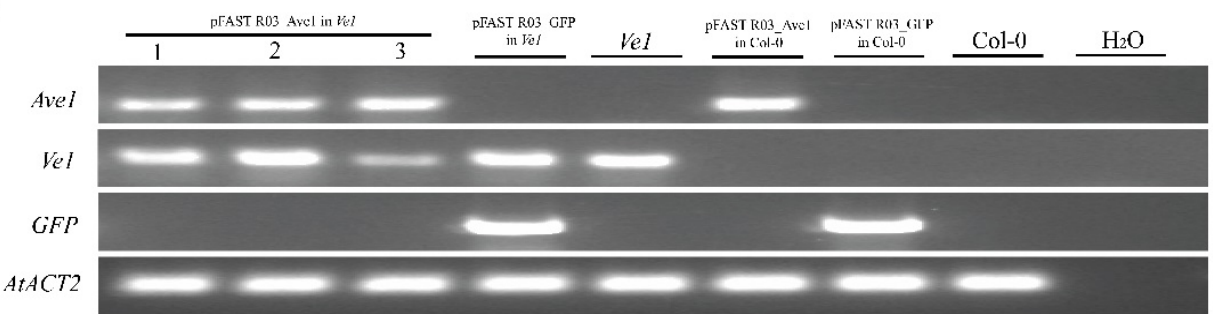

B

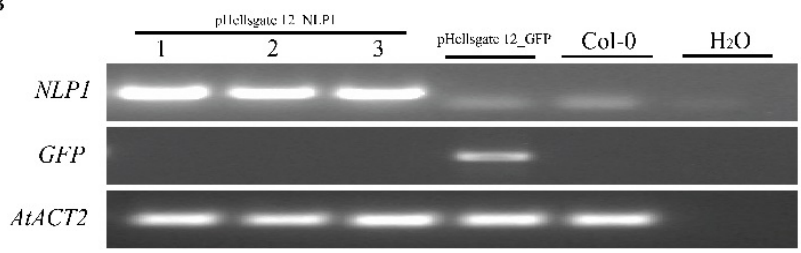

C

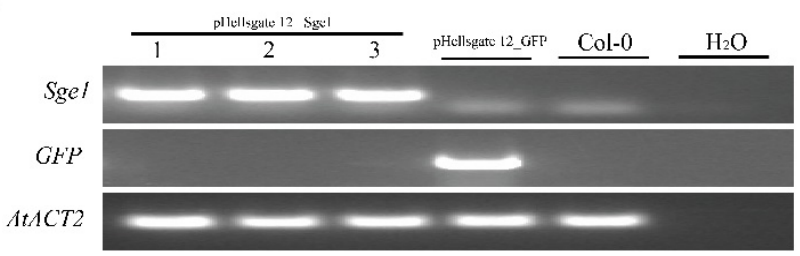

Figure S2. Polymerase chain reaction (PCR) amplification from cDNA of the corresponding transgenic Arabidopsis thaliana lines. (A) Transcripts for hairpin Ave1 or GFP formation in transgenic lines were detected by reverse transcription-PCR (RT-PCR). For pFAST R03_Ave1 construct in Ve1 plants three independent transgenic lines are shown (1, 2 and 3), while other lines expressing pFAST R03_Ave1 construct or pFAST R03_GFP construct are shown as controls. (B) Transcripts for hairpin NLP1 or GFP formation in transgenic lines were PCR-detected. For pHellsgate 12_NLP1 construct in Col-0 plants three independent transgenic lines are shown (1,2 and 3), and the corresponding control line expressing pHellsgate 12_GFP construct is shown. (C) Transcripts for hairpin NLP1 or GFP formation in transgenic lines were PCR-detected. For pHellsgate 12_Sge1 construct in Col-0 plants three independent transgenic lines are shown (1, 2 and 3), and the corresponding control line expressing pHellsgate 12_GFP construct is shown. As an endogenous control, a fragment of the AtACTIN2 gene was amplified from A. thaliana cDNA. A. thaliana Col-0 and water are used as RT-PCR controls. 
HIGS compromises Verticillium wilt in tomato and Arabidopsis

Table S1. Primers used in this study.

\begin{tabular}{|c|c|c|}
\hline Primer name & Oligonucleotide sequence $\left(5^{\prime} \rightarrow 3^{\prime}\right)$ & Description $^{\mathrm{a}}$ \\
\hline Ave1-F & ggggacaagtttgtacaaaaaagcaggctATGAAGCTTTCTACGCTT & Ave1 (RNAi) \\
\hline Ave1-R & ggggaccactttgtacaagaaagctgggtTATCTGTCTAAATTC & Ave1 (RNAi) \\
\hline Ave1-F(RT) & AGCTTTCTACGCTTGGA & RT-PCR \\
\hline Ave1-R(RT) & TTGGCTGGGATTGCT & RT-PCR \\
\hline NLP1-F & ggggacaagtttgtacaaaaaagcaggctGGTCTCCAAGACAGCGGTAC & NLP1 (RNAi \& RT-PCR) \\
\hline NLP1-R & ggggaccactttgtacaagaaagctgggtGTTTGCGTTGTTCGAGTTGA & NLP1 (RNAi \& RT-PCR) \\
\hline Sge1-F & ggggacaagtttgtacaaaaaagcaggctCCTGTCTGGCAATCTTCGTC & Sge1 (RNAi \& RT-PCR) \\
\hline Sge1-R & ggggaccactttgtacaagaaagctgggtCCGTTGGTCTGCTTCTTGTT & Sge1 (RNAi \& RT-PCR) \\
\hline GFP-F & ggggacaagtttgtacaaaaaagcaggctGTGGAGAGGGTGAAGGTGA & GFP (RNAi \& RT-PCR) \\
\hline GFP-R & ggggaccactttgtacaagaaagctgggtAAGGGCAGATTGTGTGGAC & GFP (RNAi \& RT-PCR) \\
\hline TRV2_CP-F & CTGCGAATCCAAACACAAC & Coat protein \\
\hline TRV2_CP-R & GAACCGCCTGAACATAAAAA & Coat protein \\
\hline ITS1-F & AAAGTTTTAATGGTTCGCTAAGA & V. dahliae (fungal biomass; qPCR) \\
\hline STVe1-R & CTTGGTCATTTAGAGGAAGTAA & V. dahliae (fungal biomass; qPCR) \\
\hline SlActin-F & CCATTCTCCGTCTTGACTTGG & Tomato actin (fungal biomass; qPCR) \\
\hline SlActin-R & TCTTTCCTAATATCCACGTCAC & Tomato actin (fungal biomass; qPCR) \\
\hline AtRubisco-F & GCAAGTGTTGGGTTCAAAGCTGGTG & Arabidopsis Rubisco (fungal biomass; qPCR) \\
\hline AtRubisco-R & CCAGGTTGAGGAGTTACTCGGAATGCTG & Arabidopsis Rubisco (fungal biomass; qPCR) \\
\hline Sge1-F(qRT) & CACCGAATTACGCTCAACCT & V. dahliae (RT-qPCR) \\
\hline Sge1-R (qRT) & AAGTCATAGGCCGTGGAGTG & V. dahliae (RT-qPCR) \\
\hline VdGAPDH-F & CGAGTCCACTGGTGTCTTCA & V. dahliae (RT-qPCR) \\
\hline VdGAPDH-R & CCCTCAACGATGGTGAACTT & V. dahliae (RT-qPCR) \\
\hline Ve1-F(RT-PCR) & CATATTGAAATTAGCGTCTTGTCGG & Tomato Ve1 (RT-PCR) \\
\hline Ve1-R(RT-PCR) & ACCGAGAAAAAGGAGGCAAAAC & Tomato Ve1 (RT-PCR) \\
\hline AtACT2-F & GATGGAGACCTCGAAAACCA & Arabidopsis actin (RT-PCR) \\
\hline AtACT2-R & AAAAGGACTTCTGGGCACCT & Arabidopsis actin (RT-PCR) \\
\hline
\end{tabular}

aThe type of experiment for which the primers were used is indicated in brackets (RNAi, RNA interference; RT-PCR: Reverse TranscriptionPCR; qPCR, quantitative Real Time-PCR; RT-qPCR: Reverse Transcription-qPCR). 



\section{Chapter 7}

\section{General discussion}

A modified version of this chapter has been published as:

Rodriguez-Moreno L\#, Song Y\#, Thomma BPHJ (2017) Transfer and engineering of immune receptors to improve recognition capacities in crops. Curr Opin Plant Biol 38: 42-49. (\#equal contribution) 


\section{Chapter 7}

\section{ABSTRACT}

Immune receptors are pivotal elements of the plant immune system that act as sentinels for microbial invasion. Knowingly or unknowingly, breeding for resistance has largely relied on the transfer of immune receptor recognition specificities between plant genotypes. For decades such transfers were limited to crossable species. However, advents in transgene technologies have allowed overcoming species barriers. Novel strategies for mining of recognition specificities, combined with our recently increased understanding of immune receptor functioning, allows to increase and alter recognition specificities, which should ultimately increase the spectrum of recognition specificities that are available to control plant diseases in crops. 


\section{INTRODUCTION}

Plant diseases, caused by pathogens and pests, threaten food security and food safety worldwide and remain a major agricultural challenge. Like any other organism, plants evolved innate immune systems that act against pathogen attack, and which are continuously tweaked in the ongoing arms races with a wide range of microbes (Dodds and Rathjen, 2010; Thomma et al., 2011; Cook et al., 2015). Central to the immune system are receptors that detect pathogen invasion through sensing pathogen(-induced) ligands, also termed invasion patterns, to mount appropriate immune responses (Cook et al., 2015). Based on structure and subcellular location, these invasion pattern receptors fall into two major classes; cell surface localized receptors that comprise receptor kinases (RKs) and receptorlike proteins (RLPs) that monitor the extracellular space, while cytoplasm-localized nucleotide-binding domain leucine-rich repeat receptors (NLRs) survey the intracellular environment.

For decades, breeding for resistance has relied on the identification of resistance or of recognition traits in genotypes of crop species or their (wild) relatives followed by introduction into elite cultivars by crossing. For example, Verticillium wilt resistance in many modern cultivated tomato (Solanum lycopersicum) varieties is based on the introgression of the dominant Ve locus that was identified in the tomato accession Peru Wild in the 1930s (Schaible et al., 1951). Meanwhile, our mechanistic understanding of pathogen recognition has increased significantly (Zhang and Thomma, 2013; Cui et al., 2015; Li et al., 2016). For instance, research over the last decades has shown that race-specific resistance to Verticillium wilt in tomato is governed by the cell surface-localized RLP-type immune receptor Ve1 encoded by the Ve locus (Kawchuk et al., 2001; Fradin et al., 2009) through recognition of the effector protein Ave1 that is secreted by race 1 strains of Verticillium spp. (de Jonge et al., 2012). In this thesis we further propose that a surface-exposed patch of the Ave1 protein that is composed of co-localized Nand C-termini is recognized by tomato Ve1 (Chapter 3). These advances spur the identification and deployment of novel recognition specificities to enhance disease resistance in crops. 


\section{Interfamily transfer of immune receptors to confer disease resistance}

Since the ground-breaking finding that introduction of the cell surface-localized elongation factor Tu receptor (EFR) from the crucifer Arabidopsis thaliana into the Solanaceous plants Nicotiana benthamiana and tomato confers responsiveness to bacterial elongation factor Tu and bacterial resistance (Lacombe et al., 2010), it has become apparent that immune receptor genes may be transferred between plant families to engineer disease resistance (Figure 1a; Table 1). In this thesis, we show that homologs of tomato Ve1 are widespread in the plant kingdom (Chapter 4), and that tomato Ve1 can be functionally transferred to crops such as tobacco and cotton (Chapter 5). Successful transfers have even been reported across the dicot and monocot clades with, for example, transfer of the barley NLR Mildew Resistance Locus $A$ (MLA1) to A. thaliana induce immunity against barley powdery mildew expressing the corresponding effector AVRA1 (Maekawa et al., 2012). Arguably, examples like these imply that signalling components downstream of immune receptors are sufficiently conserved among plant species, families and even clades to mount the appropriate defence responses. Currently, most successful interfamily transfers of immune receptors have been reported for cell surface receptors (Table 1), which again also include transfer of tomato Ve1, initially to the model plant $A$. thaliana (Fradin et al., 2009), and in this thesis to the crop species tobacco and cotton (Chapter 5). Potentially, this is because this type of immune receptor participates in receptor complexes that generally require the same, highly conserved, receptor-associated kinases to mount downstream immune responses (Liebrand et al., 2014). The functionality of many, if not all, RLPs carrying extracellular leucine-rich repeats (eLRRs), requires constitutive association with the common adaptor kinase Suppressor of BAK1-Interacting Receptor-like Kinase 1 (SOBIR1) in order to constitute a bimolecular equivalent of a genuine RK (Gust and Felix, 2014). This obviously also applies to RLP-type immune receptor Ve1 (Liebrand et al., 2013). Moreover, RKs as well as RLP/SOBIR1 complexes interact in

a ligand-dependent manner with the Brassinosteroid Insensitive 1 (BRI1)Associated receptor Kinase 1 (BAK1) for immune signalling activation. Although physical recruitment of BAK1 upon Ave1-mediated activation of the Ve1-SOBIR1 complex has not yet been demonstrated, genetic requirement of $B A K 1$ for the Ave1- 
induced Ve1-mediated immune response has previously been demonstrated in both tomato and $A$. thaliana (Fradin et al., 2009; 2011). Although BAK1 also participates in receptor complexes that mediate developmental processes, it has recently been proposed that immune and developmental signalling complexes show spatiotemporal separation in plasma membrane nanodomains, which might mechanistically explain dual BAK1 functionality (Bücherl et al., 2017). Importantly, SOBIR1 and BAK1 are widely conserved in the plant kingdom (Liebrand et al., 2014).

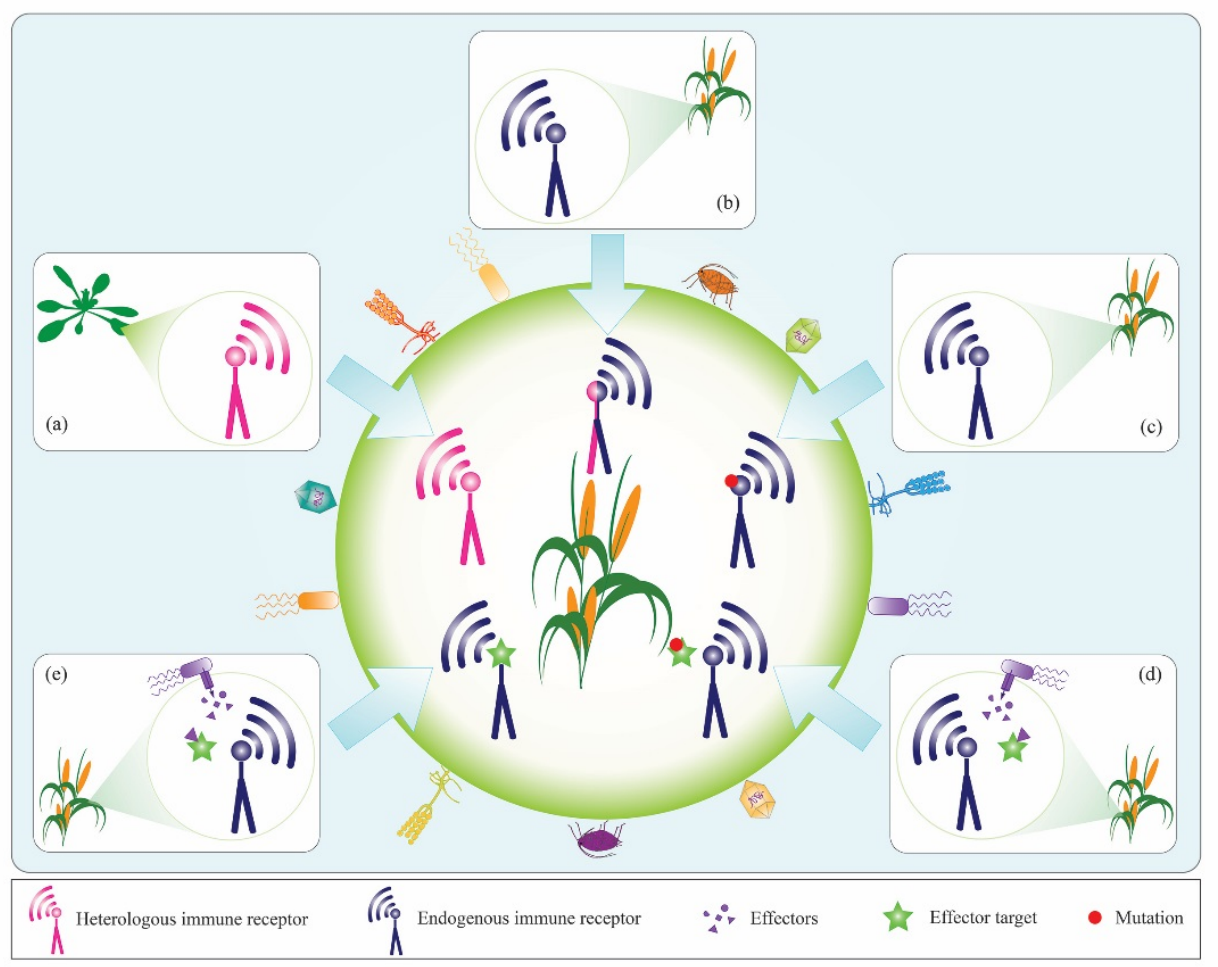

Figure 1. Strategies for plant immune receptor deployment to improve pathogen recognition capacities in crops. (a) Transfer of an immune receptor (displayed as an antenna) from another plant species to engineer disease resistance in the target crop (in the centre of the circle). (b) Generation of an inter-species chimeric immune receptor to engineer or alter pathogen recognition specificity. (c) Mutation (red dot) of an endogenous immune receptor to alter or enhance recognition specificity. (d) Mutation (red dot) of an effector target (green star) to expand immune receptor recognition capacity. (e) Integration of a decoy effector target (green star) into an immune receptor to engineer pathogen recognition. 


\section{Chapter 7}

Table 1 Examples of functional transfer of plant immune receptor genes between families.

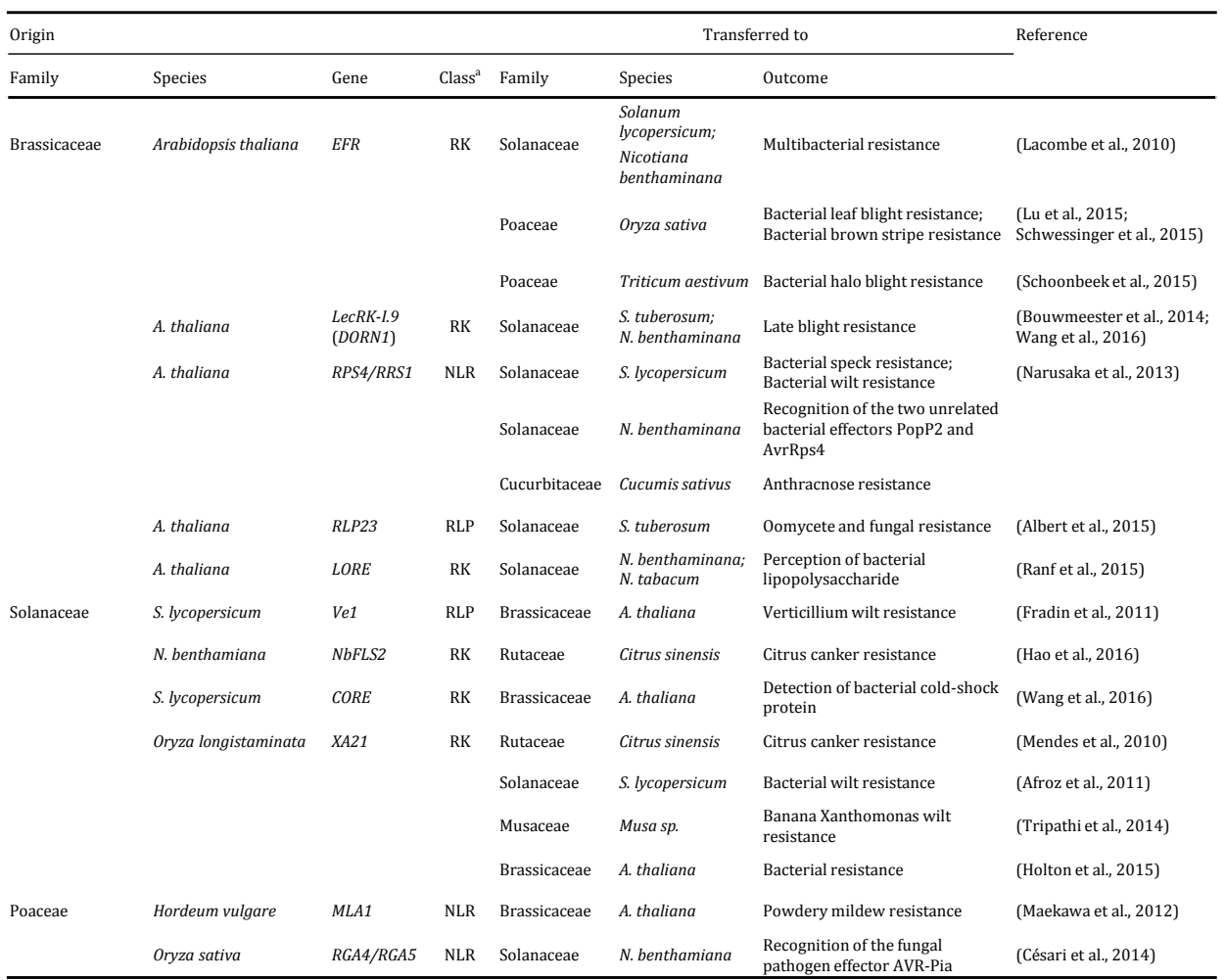

${ }^{a}$ RK, receptor kinase; RLP, receptor-like protein; NLR, nucleotide-binding domain leucine-rich repeat receptor.

Functional transfer of NLR-type immune receptors between taxonomically distinct families has met with relatively little success when compared with the transfer of cell surface receptors. Potentially this indicates that the molecular components that they need to interact with are less conserved. However, it is also increasingly becoming apparent that NLR-type immune receptors may function in heterologous pairs to activate immunity (Eitas and Dangl, 2010; Okuyama et al., 2011; Yuan et al., 2011; Brotman et al., 2013; Cesari et al., 2013; Wang et al., 2013). In support of this, transfer of the Arabidopsis NLR pair Resistance to Pseudomonas syringae 4 (RPS4) with Resistance to Ralstonia solanacearum 1 (RRS1) allows recognition of the PopP2 and AvrRps4 effectors of Ralstonia solanacearum and Pseudomonas syringae, respectively, in $N$. benthamiana and confers resistance against these bacterial pathogens in tomato (Narusaka et al., 2013). Moreover, transgenic cucumber lines expressing RPS4-RRS1 are resistant against 
Colletotrichum orbiculare, although it is not clear what effector is recognized in this case (Narusaka et al., 2013).

\section{Exploitation of the modular nature of immune receptors to extend pathogen recognition specificities}

Structurally, most immune receptors are modular proteins with differentiated domains for ligand recognition and for intracellular signalling. Conceivably, ligand perception by extracellular receptors is mostly determined by the eLRR domain, while intracellular signalling is mostly conferred by the cytoplasmic kinase domain (for RKs) or interaction with co-receptor SOBIR1 (for RLPs) (Couto and Zipfel, 2016). Such modular composition offers opportunities to engineer chimeras that are composed of domains from different receptors (Figure 1b). The first report of a functional chimeric plant receptor stems from the year 2000, claiming that a chimeric receptor consisting of the extracellular domain of the Arabidopsis RK BRI1, which perceives brassinosteroids to activate developmental signalling, fused to the kinase domain of the rice RK XA21, an immune receptor involved in bacterial blight resistance, mediates immune activation upon brassinosteroid treatment (He et al., 2000). Similarly, domain swaps between the tomato RLP Ve1 and its closely nonfunctional homolog Ve2 allowed construction of artificial receptors carrying Cterminal part of $\mathrm{Ve} 2$ replaced by that of Ve1 to mediate Verticillium resistance through perception of the effector protein Ave1 (Fradin et al., 2014). More recently, chimeras between Arabidopsis EFR and rice XA21 confirmed that the XA21 kinase domain, despite being derived from a monocot, associates with intracellular components of the EFR complex in the dicot Arabidopsis to activate immunity (Holton et al., 2015). Similarly, rice transformants expressing EFR, or EFR:XA21 chimeras, respond to Escherichia coli and Xanthomonas oryzae pv. oryzae elf18 (Schwessinger et al., 2015). In addition to reinforcing the notion that signalling components downstream of the receptors are widely conserved, these examples illustrate the modular nature of immune receptors.

NLRs consist of an N-terminal coiled-coil (CC) or Toll-interleukin (TIR) domain, a central ATPase nucleotide-binding site (NBS), and C-terminal LRRs. Although the high degree of polymorphism of the LRR domains suggests involvement in ligand binding (Krasileva et al., 2010), also the CC-domain was found to interact with 


\section{Chapter 7}

ligands (Chen et al., 2012). Switching from a non-active to an active state is controlled by intramolecular rearrangements within the TIR/CC and NBS subdomains associated with nucleotide exchange (Tameling et al., 2002; Bernoux et al., 2016). Domain swaps between closely related yet polymorphic proteins frequently lead to either inactive or constitutively active receptors (Slootweg et al., 2013; Steinbrenner et al., 2015; Wang et al., 2015). Thus, exploitation of the modular nature of NLRs is less straightforward than for RKs, also because the mechanism by which they exactly function is less well understood. This is illustrated by the surprising finding that the TIR-only protein "Response to the bacterial type III effector protein HopBA1" (RBA1) lacks all other canonical NLR domains, but successfully induces immunity to P. syringae (Nishimura et al., 2017). Nevertheless, natural chimeric immune receptors exist as so-called NLRs with integrated domains (NLR-IDs). These NLR-IDs comprise non-canonical "decoy" domains that mimic effector target proteins, and act as a trap for pathogen effectors to activate immunity (Cesari et al., 2014; Wu et al., 2015). Functional analyses of NLR-IDs suggest these receptors act in pairs with conventional NLR partners, where the NLR-ID works as pathogen "sensor" while the conventional NLR acts as immune "activator" (Cesari et al., 2014). Examples of receptor pairs containing integrated decoy domains are the Arabidopsis pair RRS1 with RPS4, where RRS1 contains a decoy WRKY domain that is targeted by two unrelated bacterial effectors PopP2 and AvrRps4 (Le Roux et al., 2015; Sarris et al., 2015), and the rice pairs Resistance Gene Analog 5 (RGA5) with RGA4 (Césari et al., 2014) and Pyricularia oryzae resistance-k 1 (Pik-1) with Pik-2 (Maqbool et al., 2015), where RGA5 and Pik-1 contain heavy metal-associated (HMA) domains that are targeted by the AVR1-C039, AVR-Pik and AVR-Pia effectors of the rice blast fungus Magnaporthe oryzae (Cesari et al., 2013; Maqbool et al., 2015; Ortiz et al., 2017). Interestingly, the positioning of the IDs within the overall structure of NLR-IDs suggests structural plasticity, with the decoy domain integrated between the CC- and NBS domains, e.g. in Pik-1, or at the C-terminus of the NLR, e.g. in RRS1 and RGA5. NLR-IDs are widely distributed across all lineages of flowering plants (Kroj et al., 2016; Sarris et al., 2016), and recent studies on grass genomes suggest the presence of genomic hotspots containing flexible NLR-"acceptor" genes that may integrate other domains from across the genome (Bailey et al., 2017). 


\section{Artificial evolution to extend immune receptor recognition}

The identification of novel pathogen recognition specificities is highly desired by plant breeders. Besides screening for novel specificities in natural collections, also artificial evolution through random or targeted mutagenesis has successfully been exploited to expand the recognition spectrum of plant immune receptors. The NLR $\mathrm{Rx}$ confers resistance against a single strain of potato virus X (PVX) by recognising its capsid protein (CP). Rx can recognise PVX isolates containing $\mathrm{T}$ and $\mathrm{K}$ at positions 121 and 127, respectively ( $\mathrm{CP}_{\mathrm{TK}}$ ), but not those with $\mathrm{K}$ and $\mathrm{R}$ at these positions ( $\mathrm{CP}$ KR). Based on random mutagenesis of the LRR domain, Rx variants that also responded to $\mathrm{CP}_{\mathrm{KR}}$ were generated (Farnham and Baulcombe, 2006). Furthermore, one of the selected Rx mutants, RxM1, induced systemic necrosis when plants were challenged with poplar mosaic virus (PopMV), a distant relative of PVX, due to weak recognition of the PopMV CP. In a second round of random mutagenesis, five amino acid changes near the conserved ATPase nucleotidebinding pocket increased sensitivity of RxM1 towards the PopMV-CP (Harris et al., 2013).

A similar approach was followed to alter the recognition specificities of the potato NLR R3a that only recognizes the AVR3a ${ }^{\mathrm{KI}}$ allele of Phytophthora spp., while the other major allele, AVR3a ${ }^{\mathrm{EM}}$, is only weakly recognized. Using random mutagenesis eight amino-acid substitutions were identified that expand R3a recognition to include AVR3a ${ }^{\mathrm{EM}}$. Strikingly, particular mutations in the CC (R3a $\left.{ }^{\mathrm{I} 148 \mathrm{~F}}\right)$, which resulted in an auto-active R3a variant, and in the NBS (R3a ${ }^{\mathrm{N} 336 \mathrm{Y}}$ ) extended recognition to another AVR3a homolog from P. capsici (Segretin et al., 2014). The tomato R3a ortholog I2 confers resistance to Fusarium oxysporum f. sp. lycopersici through recognition of the AVR2 effector, but only weakly responds to AVR3a. Mutation of the amino acid position in I2 (I2 $\left.{ }^{\mathrm{I} 141 \mathrm{~N}}\right)$ of which the homologous position in R3a was found to generate an auto-active phenotype not only resulted in recognition of two AVR2 effector variants that previously escaped I2 recognition, but also resulted in recognition of AVR3a ${ }^{\mathrm{KI}}$ and AVR3a ${ }^{\mathrm{EM}}$ (Giannakopoulou et al., 2015).

Rather than based on random mutagenesis, structure-guided mutation of immune receptors holds promise for extension or alteration of recognition specificities (Figure 1c). Presently, an increasing number of studies report on the 


\section{Chapter 7}

crystal structure of immune receptors, sometimes together with their ligands, allowing the identification of residues involved in ligand interaction (Liu et al., 2012; Sun et al., 2013; Tang et al., 2015), and thus also the rational design of receptor modifications to alter recognition specificities. For instance, analysis of the crystal structure of the ectodomain of the Arabidopsis RK Flagellin Sensing 2 (FLS2) together with the bacterial flagellin (flg22) ligand revealed that the LRR forms a solenoid structure whose inner surface interacts with both the N-terminus and Cterminus of flg22 (Sun et al., 2013). Targeted mutagenesis extended FLS2 recognition capacities towards new flg22 ligands for which the wild-type FLS2 confers little or no response (Helft et al., 2016). As CRISPR/Cas systems are further refined and developed for more cropping systems (Puchta, 2017), structure-guided engineering of immune receptors will likely become customary.

\section{Modification of effector targets to expand receptor recognition capacities}

Pathogen detection by immune receptors may occur directly by physical interaction with pathogen-derived or induced molecules, or indirectly according to the guard model by detecting effector target modifications (Van Der Biezen and Jones, 1998). The latter mechanism in particular offers the possibility to engineer host targets to be recognized by immune receptors that previously did not respond to the effector protein (Figure 1d). The P. syringae AvrPphB effector is a protease that cleaves the host kinase PBS1 (for Avirulence Protein Pseudomonas phaseolicola B (avrPphB) Susceptible 1) that functions in basal immunity, which is monitored by the Arabidopsis NLR RPS5 (Shao et al., 2003). Engineering of PBS1 such that it is cleaved by proteases of other pathogens results in extension of disease resistance mediated by RPS5 (Kim et al., 2016).

Members of the genus Xanthomonas and Ralstonia secrete transcription activator-like effectors (TALEs) that bind specific DNA sequences, known as effector binding elements (EBEs), to activate host target genes (Kay et al., 2007; Boch et al., 2009; Moscou and Bogdanove, 2009). In turn, plants evolved "executor" genes with EBEs embedded in the promoters that induce cell death and resistance (Römer et al., 2007). Based on "executor" sequences, novel EBEs can be engineered 
that trigger immunity upon invasion of pathogens that inject TALEs (Römer et al., 2009; Hummel et al., 2012; Zeng et al., 2015).

Already 25 years ago, a so-called two-component sensor system was proposed to engineer resistance against a broad range of (obligate) biotrophic pathogens by transgenic expression of corresponding pairs of effectors and immune receptors under control of (preferably two different), pathogen-inducible promoters (de Wit, 1992). Bacterial leaf streak of rice is caused by X. oryzae pv. oryzicola, yet no resistance against this pathogen is known. Resistance was established in transgenic rice lines harbouring the M. oryzae AVR1-CO39 effector gene under the control of an inducible promoter with an embedded EBE when the corresponding Pi-CO39 resistance locus was present (Hutin et al., 2016). In an alternative strategy, a similar transactivation of an auto-active NLR by a TAL effector of the $X$. oryzae pv. oryzae triggered bacterial blight resistance (Hutin et al., 2016). Such approaches based on synthetic promoter "traps" widely expand the panel of genes that can be exploited to engineer resistance in plants that suffer from infections by TALEinjecting pathogens.

\section{Future engineering of disease resistance in crops through the deployment of immune receptors}

Securing sustainable food production remains a challenge, as pathogens continuously adapt to overcome host immunity and can emerge on novel crop species, such as the recent wheat blast outbreak in Bangladesh and India (Malaker et al., 2016). This is particularly problematic when resistance sources are scarce. The fungus Phakopsora pachyrhizi causes Asian soybean rust (ASR) that is one of the most economically important crop diseases and mostly controlled by chemicals as no effective genetic resistance occurs in cultivars, yet fungicide resistance is an emerging problem for this pathogen. Transgenic expression of the recently identified NLR CcRpp1 from the legume weed pigeonpea (Cajanus cajan) confers full resistance to ASR in soybean (Kawashima et al., 2016), showing how (wild) relatives may provide resistance traits for crop improvement.

In addition to identification of recognition specificities based on screening for pathogen resistance, functional genomics approaches based on effector recognition ("effectoromics") can be used for probing plant germplasm for recognition 
specificities (Vleeshouwers and Oliver, 2014). This is especially powerful as it also allows screening for recognition specificities in plant species that are not hosts for the pathogen for which recognition is sought. Moreover, an effectoromics strategy allows combination with pathogenomics strategies to select the potentially most widespread or invariant effectors to, once identified and deployed, maximize durability of the recognition specificity (Bart et al., 2012). However, the subsequent cloning of immune receptor genes is typically laborious and slow, but functional genomic approaches based on NLR gene enrichment and sequencing (RenSeq), especially when combined with long-read sequencing, can accelerate mapping of functional NLRs in crop plants (Jupe et al., 2013; Witek et al., 2016), as well as in wild relatives (Stam et al., 2016).

An interesting strategy has been proposed to identify cell surface receptors particularly based on their general ligand-induced association with the "promiscuous" co-receptor BAK1. Using affinity-tagged BAK1 as molecular bait, a $N$. benthamiana eLRR-RLP-type cell surface receptor was recently identified as NbCSPR that is involved in the response to a bacterial cold-shock peptide (csp22) (Saur et al., 2016). Intriguingly, based on natural variation in csp22 perception an RK-type cell surface receptor was identified in tomato as high-affinity receptor for csp22 and named CORE, which has homologs in several other Solanaceous plants, including $N$. benthamiana (Wang et al., 2016). The latter study attempted to reconcile the role of NbCSPR and NbCORE in csp22 perception but failed to demonstrate a role for NbCSPR in csp22 responsiveness, suggesting that NbCSPR is neither sufficient nor necessary for csp22 perception. Further studies will have to clarify this apparent discrepancy, which should then also validate the strategy of using BAK1 as molecular bait to identify cell surface receptors.

A recent discovery indicated that approximately $10 \%$ of the NLRs belong to the ID-class with integrated decoys, and thus far a few hundred unique integrated domains have been identified (Kroj et al., 2016; Sarris et al., 2016). Interestingly, there is a significant overlap between integrated domains fused to plant NLRs and protein domains found to frequently interact with pathogen effectors in massive yeast two-hybrid screens against A. thaliana proteins (Mukhtar et al., 2011; Weßling et al., 2014; Sarris et al., 2016). Overlapping domains include putative kinase domains, DNA binding domains (e.g. the WRKY domain), and proteins 
involved in redox reactions (e.g. the HMA domain) as well as hormone signalling and cytoskeleton (Sarris et al., 2016). Obviously, these molecules may be screened for activation by pathogen effectors. Indeed, this idea was tested by focussing on a particular type of NLR-ID. Multiple NLRs have integrated a predicted DNA-binding BED zinc finger domain between their CC and NBS domains (Kroj et al., 2016). The authors tested a rice NLR-type gene that encodes a ZBED protein containing three $\mathrm{N}$-terminally integrated BED zinc finger domains, showing that ZBED-expressing rice plants were more resistant to rice blast fungus $M$. oryzae while ZBED-deficient plants were more susceptible (Kroj et al., 2016). Although recognition of pathogen effectors by BED domains in ZBED protein remains to be established, this test indicates that characterization of integrated domains in NLRs can serve as an extremely powerful way for identification of new players in plant immunity. Moreover, making use of this plasticity in NLR-IDs, novel variants may be engineered upon integration of other "pathogen-customized" domains, based on interactors that can be identified for one's favourite effector (Figure 1e).

Transfer and artificial evolution of plant immune receptors have successfully been deployed to improve pathogen recognition capacities (Table 1; Farnham and Baulcombe, 2006; Harris et al., 2013; Chapman et al., 2014; Segretin et al., 2014; Giannakopoulou et al., 2015; Helft et al., 2016). These strategies can also be utilized to exploit known immune receptors for engineering disease resistance in crops. In this case, homologues of Verticillium effector Ave1 were previously identified in a number of pathogenic microbes, including the citrus bacterial canker pathogen Xanthomonas. axonopodis pv. citri (XacPNP), the fungal tomato wilt pathogen $F$. oxysporum f. sp. lycopersici (FoAve1), the fungal sugar beet leaf spot pathogen Cercospora beticola (CbAve1), the crucifer anthracnose pathogen Colletotrichum higginsianum (ChAve1) and the cucurbit anthracnose pathogen Colletotrichum orbiculare (CoAve1) (Nembaware et al., 2004; de Jonge et al., 2012; Gan et al., 2013), and these homologs are differentially recognized by tomato Ve1 and its functional homologues (de Jonge et al., 2012; Chapter 3 and Chapter 4). It was shown that Ve1 mediates resistance against $F$. oxysporum f. sp. lycopersici in tomato, demonstrating involvement of this immune receptor in resistance against multiple fungal pathogens. And tomato $\mathrm{Ve} 1$ can be functionally transferred to the closely related plant species (Nicotiana tabacum) as well as to the distantly related plant species ( $A$. 


\section{Chapter 7}

thaliana and cotton), resulting in enhanced Verticillium wilt resistance in an Ave1dependent manner (Fradin et al., 2011; de Jonge et al., 2012; Chapter 5). Thus, these findings inspire us to further exploit tomato Ve1 (homologues) for engineering disease resistance in an Ave1(homolog)-dependent manner, for example through transfer or experimental evolution of tomato Ve1(homologues).

Recently, Kim et al. (2016) took advantage of the indirection recognition mechanism to engineer a host effector target to increase novel recognition specificities by trapping unrelated pathogen-derived proteases in their act. Engineering host effector target strategy may be applicable to other immune receptors to expand their recognition capacities. Besides monitoring of host effector target cleavage, sensing of inhibition, phosphorylation and other modification of guarded effector targets through immune receptors have also been reported (Rooney et al., 2005; van Esse et al., 2008; Song et al., 2009; Liu et al., 2011; Lozano-Torres et al., 2012; Innes, 2015; Duxbury et al., 2016). For example, the tomato cell surface-localized RLP-type immune receptor Cf2 monitors the interaction of the apoplastic tomato cysteine protease Rcr3 with the effectors Avr2 and Gr-VAP1, protease inhibitors secreted by the tomato leaf mould fungus Cladosporium fulvum (Dixon et al., 2000; Rooney et al., 2005) and by the obligate plant-parasitic nematode Globodera rostochiensis, respectively (Lozano-Torres et al., 2012).

\section{Auxiliary strategies for developing disease resistance in crops}

Besides deployment of immune receptors, there are a number of other approaches for developing disease resistance in crops. Recessive disease resistance genes are commonly regarded as disease susceptibility $(S)$ genes that encode host proteins required for pathogen survival and proliferation. Host genes encoding such susceptible factors are promising candidates for engineering disease resistance through loss-of-function of the host susceptible factors, as inactivation of host susceptible factors can limit pathogen survival and growth (Pavan et al., 2010; Gawehns et al., 2013; van Schie and Takken, 2014). Indeed, loss-of-function of the Mildew resistance locus o (Mlo) in barley was found to confer recessively inherited broad-spectrum resistance against powdery mildew (Jørgensen, 1992). Barley Mlo is a plasma membrane-localized protein that is required for pathogen invasion 
(Humphry et al., 2006; Kusch and Panstruga, 2017). Notably, mlo-mediated powdery mildew resistance has also been described in other plant species, including A. thaliana, tomato, pea, wheat, apple, grapevine, pepper, rose, tobacco, cucumber and melon (Kusch and Panstruga, 2017). Other successful examples of recessive resistance genes are the eukaryotic translation elongation initiation factor genes eif4e and eif $4 \mathrm{~g}$, conferring potyvirus resistance in lettuce, melon, tomato, pepper, barley, pea and rice (Wang and Krishnaswamy, 2012), and rice xa13, xa25 and Os11N3 (also named as OsSWEET11, OsSWETT13 and OsSWEET14, respectively), conferring bacterial blight resistance against $X$ oryzae pv. oryzae (Zhang and Wang, 2013). Notably, the application of mutated alleles of host $S$ genes in breeding programmes can be problematic if pleiotropic effects, such as dwarfism or spontaneous necrotic lesions, are observed in loss-of-function mutants of $S$ genes (van Schie and Takken, 2014).

RNA interference (RNAi) is a widely conserved regulatory mechanism that affects gene expression in eukaryotic organisms, and cross-kingdom RNAi and small RNA (sRNA) trafficking have been described in plant-pathogen interactions (Knip et al., 2014; Weiberg and Jin, 2015). Cross-kingdom RNAi from a host plant to its interacting pathogens or pests is called host-induced gene silencing (HIGS), a phenomenon in which a host plant-generated sRNA triggers silencing of a pathogen gene (Nunes and Dean, 2012; Koch and Kogel, 2014). Although the HIGS approach has been examined in multiple crops to combat plant diseases caused by various pathogens (Nunes and Dean, 2012; Koch and Kogel, 2014; Wang et al., 2017), it has not yet been applied in breeding programmes to control plant diseases. In this thesis, we also assessed whether HIGS can be utilized to control Verticillium wilt disease by silencing virulence genes of Verticillium dahliae through the host plants tomato and A. thaliana (Chapter 6). Our results clearly show that plants transiently (in tomato) or stably (A. thaliana) expressing RNAi constructs targeting transcripts of genes that are essential for $V$. dahliae pathogenicity can become protected from Verticillium wilt disease, suggesting that HIGS against $V$. dahliae may be exploited to engineer Verticillium wilt resistance in crops (Chapter 6). Indeed, a recent report on HIGS protecting Verticillium wilt-susceptible cotton plants from $V$. dahliae infection has been described (Zhang et al., 2016). Considering that HIGS is limited by a ban on growing genetically modified (GM) crops in large regions of the world, 


\section{Chapter 7}

and the instability of engineered RNA silencing traits, an alternative sRNA trafficking-based disease control approach called spray-induced gene silencing (SIGS) is proposed in which spaying sRNAs that target essential pathogen genes on plant surfaces confer effective crop protection (Wang and Jin, 2017). SIGS has recently been tested for controlling Fusarium graminearum infection on barley (Koch et al., 2016), inhibiting Botrytis cinerea on vegetables, fruits and flower petals (Wang et al., 2016), and protecting cowpea and tobacco from plant viruses (Mitter et al., 2017). sRNA trafficking-based disease control approaches could be alternatives for developing disease resistance in crops for which no natural resistance source is available.

\section{CONCLUDING REMARKS}

Ultimately, once it is fully understood how ligand specificity and signal transduction activation by immune receptors is biochemically established, we may be able to design complete immune receptors de novo. Potentially, this allows designing a spectrum of immune receptors for any given pathogen once we know what molecules are exposed during host colonization. Presently, however, recognition specificities are scarce and should be managed wisely. Thus, strategies based on the transfer of individual immune receptor genes can be rapidly overcome under field conditions and pyramiding of immune receptors that recognize different ligands is imperative (Kim et al., 2012; Zhu et al., 2012; Jo et al., 2014; Haesaert et al., 2015; Xiao et al., 2016). Moreover, durability of the immune receptors can be further reinforced by using strategies to engineer immunity other than those exploiting immune receptors, such as editing of host susceptibility genes or exploitation of sRNA trafficking-based disease control strategies (e.g. HIGS and SIGS). 


\section{REFERENCES}

Afroz A, Chaudhry Z, Rashid U, Ali GM, Nazir F, Iqbal J, Khan MR (2011) Enhanced resistance against bacterial wilt in transgenic tomato (Lycopersicon esculentum) lines expressing the Xa21 gene. Plant Cell Tissue Organ Cult 104: 227-237.

Albert I, Böhm H, Albert M, Feiler CE, Imkampe J, Wallmeroth N, Brancato C, Raaymakers TM, Oome S, Zhang H, Krol E, Grefen C, Gust AA, Chai J, Hedrich R, Van den Ackerveken G, Nürnberger T (2015) An RLP23-SOBIR1-BAK1 complex mediates NLP-triggered immunity. Nat Plants 1: 15140

Bailey PC, Dagdas G, Baggs E, Haerty W, Krasileva KV (2017) Immune receptors with exogenous domain fusions form evolutionary hotspots in grass genomes. bioRxiv: http://dx.doi.org/10.1101/100834.

Bart R, Cohn M, Kassen A, McCallum EJ, Shybut M, Petriello A, Krasileva K, Dahlbeck D, Medina C, Alicai T (2012) High-throughput genomic sequencing of cassava bacterial blight strains identifies conserved effectors to target for durable resistance. Proc Natl Acad Sci USA 109: E1972-E1979.

Bernoux M, Burdett H, Williams SJ, Zhang X, Chen C, Newell K, Lawrence GJ, Kobe B, Ellis JG, Anderson PA (2016) Comparative analysis of the flax immune receptors L6 and L7 suggests an equilibrium-based switch activation model. Plant Cell 28: 146-159.

Boch J, Scholze H, Schornack S, Landgraf A, Hahn S, Kay S, Lahaye T, Nickstadt A, Bonas U (2009) Breaking the code of DNA binding specificity of TAL-type III effectors. Science 326: 1509-1512.

Bouwmeester K, Han M, Blanco-Portales R, Song W, Weide R, Guo LY, van der Vossen EA, Govers F (2014) The Arabidopsis lectin receptor kinase LecRK-I. 9 enhances resistance to Phytophthora infestans in Solanaceous plants. Plant Biotechnol J 12: 10-16.

Brotman Y, Normantovich M, Goldenberg Z, Zvirin Z, Kovalski I, Stovbun N, Doniger T, Bolger AM, Troadec C, Bendahmane A (2013) Dual resistance of melon to Fusarium oxysporum races 0 and 2 and to Papaya ring-spot virus is controlled by a pair of head-to-head-oriented NB-LRR genes of unusual architecture. Mol Plant 6: 235-238.

Bücherl CA, Jarsch IK, Schudoma C, Segonzac C, Mbengue M, Robatzek S, MacLean D, Ott T, Zipfel C (2017) Plant immune and growth receptors share common signalling components but localise to distinct plasma membrane nanodomains. eLife 6: 25114.

Cesari S, Bernoux M, Moncuquet P, Kroj T, Dodds PN (2014) A novel conserved mechanism for plant NLR protein pairs: the "integrated decoy" hypothesis. Front Plant Sci 5: 606.

Césari S, Kanzaki H, Fujiwara T, Bernoux M, Chalvon V, Kawano Y, Shimamoto K, Dodds P, Terauchi R, Kroj T (2014) The NB-LRR proteins RGA4 and RGA5 interact functionally and physically to confer disease resistance. EMBO J 33: 1941-1959.

Cesari S, Thilliez G, Ribot C, Chalvon V, Michel C, Jauneau A, Rivas S, Alaux L, Kanzaki H, Okuyama Y (2013) The rice resistance protein pair RGA4/RGA5 recognizes the Magnaporthe oryzae effectors AVR-Pia and AVR1-C039 by direct binding. Plant Cell 25: 1463-1481.

Chapman S, Stevens LJ, Boevink PC, Engelhardt S, Alexander CJ, Harrower B, Champouret N, McGeachy K, Van Weymers PS, Chen X (2014) Detection of the virulent form of AVR3a from Phytophthora infestans following artificial evolution of potato resistance gene $R 3 a$. PLOS ONE 9: e110158.

Chen Y, Liu Z, Halterman DA (2012) Molecular determinants of resistance activation and suppression by Phytophthora infestans effector IPI-O. PLOS Pathog 8: e1002595.

Cook DE, Mesarich CH, Thomma BPHJ (2015) Understanding plant immunity as a surveillance system to detect invasion. Ann Rev Phytopathol 53: 541-563. 
Couto D, Zipfel C (2016) Regulation of pattern recognition receptor signalling in plants. Nat Rev Immunol 16: 537-552.

Cui H, Tsuda K, Parker JE (2015) Effector-triggered immunity: from pathogen perception to robust defense. Ann Rev Plant Biol 66: 487-511.

de Jonge R, van Esse HP, Maruthachalam K, Bolton MD, Santhanam P, Saber MK, Zhang Z, Usami T, Lievens B, Subbarao KV, Thomma BPHJ (2012) Tomato immune receptor Ve1 recognizes effector of multiple fungal pathogens uncovered by genome and RNA sequencing. Proc Natl Acad Sci USA 109: 5110-5115.

de Wit PJ (1992) Molecular characterization of gene-for-gene systems in plant-fungus interactions and the application of avirulence genes in control of plant pathogens. Ann Rev Phytopathol 30: 391418.

Dixon MS, Golstein C, Thomas CM, Van der Biezen EA, Jones JD (2000) Genetic complexity of pathogen perception by plants: the example of Rcr3, a tomato gene required specifically by Cf- 2 . Proc Natl Acad Sci USA 97: 8807-8814.

Dodds PN, Rathjen JP (2010) Plant immunity: towards an integrated view of plant-pathogen interactions. Nat Rev Genet 11: 539-548.

Duxbury Z, Ma Y, Furzer OJ, Huh SU, Cevik V, Jones JD, Sarris PF (2016) Pathogen perception by NLRs in plants and animals: Parallel worlds. BioEssays 38: 769-781

Eitas TK, Dangl JL (2010) NB-LRR proteins: pairs, pieces, perception, partners, and pathways. Curr Opin Plant Biol. 13: 472-477.

Farnham G, Baulcombe DC (2006) Artificial evolution extends the spectrum of viruses that are targeted by a disease-resistance gene from potato. Proc Natl Acad Sci USA 103: 18828-18833.

Fradin EF, Abd-El-Haliem A, Masini L, van den Berg GC, Joosten MH, Thomma BPHJ (2011) Interfamily transfer of tomato Ve1 mediates Verticillium resistance in Arabidopsis. Plant Physiol 156: $2255-2265$.

Fradin EF, Zhang Z, Ayala JCJ, Castroverde CD, Nazar RN, Robb J, Liu C-M, Thomma BPHJ (2009) Genetic dissection of Verticillium wilt resistance mediated by tomato Ve1. Plant Physiol 150: 320332.

Fradin EF, Zhang Z, Rovenich H, Song Y, Liebrand TW, Masini L, van den Berg GC, Joosten MH, Thomma BPHJ (2014) Functional analysis of the tomato immune receptor Ve1 through domain swaps with its non-functional homolog Ve2. PLOS ONE 9: e88208.

Gan P, Ikeda K, Irieda H, Narusaka M, O'Connell RJ, Narusaka Y, Takano Y, Kubo Y, Shirasu K (2013) Comparative genomic and transcriptomic analyses reveal the hemibiotrophic stage shift of Colletotrichum fungi. New Phytol 197: 1236-1249.

Gawehns F, Cornelissen BJ, Takken FL (2013) The potential of effector-target genes in breeding for plant innate immunity. Microb Biotechnol 6: 223-229.

Giannakopoulou A, Steele JF, Segretin ME, Bozkurt T0, Zhou J, Robatzek S, Banfield MJ, Pais M, Kamoun S (2015) Tomato I2 immune receptor can be engineered to confer partial resistance to the oomycete Phytophthora infestans in addition to the fungus Fusarium oxysporum. Mol PlantMicrobe Interact 28: 1316-1329.

Gust AA, Felix G (2014) Receptor like proteins associate with SOBIR1-type of adaptors to form bimolecular receptor kinases. Curr Opin Plant Biol 21: 104-111.

Haesaert G, Vossen JH, Custers R, De Loose M, Haverkort A, Heremans B, Hutten R, Kessel G, Landschoot S, Van Droogenbroeck B (2015) Transformation of the potato variety Desiree with single or multiple resistance genes increases resistance to late blight under field conditions. Crop Prot 77: 163-175. 
Hao G, Pitino M, Duan Y, Stover E (2016) Reduced susceptibility to Xanthomonas citri in transgenic citrus expressing the FLS2 receptor from Nicotiana benthamiana. Mol Plant-Microbe Interact 29: $132-142$.

Harris CJ, Slootweg EJ, Goverse A, Baulcombe DC (2013) Stepwise artificial evolution of a plant disease resistance gene. Proc Natl Acad Sci USA 110: 21189-21194.

He Z, Wang Z-Y, Li J, Zhu Q, Lamb C, Ronald P, Chory J (2000) Perception of brassinosteroids by the extracellular domain of the receptor kinase BRI1. Science 288: 2360-2363.

Helft L, Thompson M, Bent AF (2016) Directed evolution of FLS2 towards novel flagellin peptide recognition. PLOS ONE 11: e0157155.

Holton N, Nekrasov V, Ronald PC, Zipfel C (2015) The phylogenetically-related pattern recognition receptors EFR and XA21 recruit similar immune signaling components in monocots and dicots. PLOS Pathog 11: e1004602.

Hummel AW, Doyle EL, Bogdanove AJ (2012) Addition of transcription activator-like effector binding sites to a pathogen strain-specific rice bacterial blight resistance gene makes it effective against additional strains and against bacterial leaf streak. New Phytol 195: 883-893.

Humphry M, Consonni C, Panstruga R (2006) mlo-based powdery mildew immunity: silver bullet or simply non-host resistance? Mol Plant Pathol 7: 605-610.

Hutin M, Césari S, Chalvon V, Michel C, Tran TT, Boch J, Koebnik R, Szurek B, Kroj T (2016) Ectopic activation of the rice NLR heteropair RGA4/RGA5 confers resistance to bacterial blight and bacterial leaf streak diseases. Plant J 88: 43-55.

Innes RW (2015) Exploiting combinatorial interactions to expand NLR specificity. Cell Host Microbe 18: 265-267.

Jo K-R, Kim C-J, Kim S-J, Kim T-Y, Bergervoet M, Jongsma MA, Visser RG, Jacobsen E, Vossen JH (2014) Development of late blight resistant potatoes by cisgene stacking. BMC Biotechnol 14: 50.

Jørgensen JH (1992) Discovery, characterization and exploitation of Mlo powdery mildew resistance in barley. Euphytica 63: 141-152.

Jupe F, Witek K, Verweij W, Śliwka J, Pritchard L, Etherington GJ, Maclean D, Cock PJ, Leggett RM, Bryan GJ (2013) Resistance gene enrichment sequencing (RenSeq) enables reannotation of the NB-LRR gene family from sequenced plant genomes and rapid mapping of resistance loci in segregating populations. Plant J 76: 530-544.

Kawashima CG, Guimarães GA, Nogueira SR, MacLean D, Cook DR, Steuernagel B, Baek J, Bouyioukos C, do VA Melo B, Tristão G (2016) A pigeonpea gene confers resistance to Asian soybean rust in soybean. Nat Biotechnol 34: 661-665.

Kawchuk LM, Hachey J, Lynch DR, Kulcsar F, van Rooijen G, Waterer DR, Robertson A, Kokko E, Byers R, Howard RJ (2001) Tomato Ve disease resistance genes encode cell surface-like receptors. Proc Natl Acad Sci USA 98: 6511-6515.

Kay S, Hahn S, Marois E, Hause G, Bonas U (2007) A bacterial effector acts as a plant transcription factor and induces a cell size regulator. Science 318: 648-651.

Kim H-J, Lee H-R, Jo K-R, Mortazavian SM, Huigen DJ, Evenhuis B, Kessel G, Visser RG, Jacobsen E, Vossen JH (2012) Broad spectrum late blight resistance in potato differential set plants MaR8 and $\mathrm{MaR} 9$ is conferred by multiple stacked $R$ genes. Theor Appl Genet 124: 923-935.

Kim SH, Qi D, Ashfield T, Helm M, Innes RW (2016) Using decoys to expand the recognition specificity of a plant disease resistance protein. Science 351: 684-687.

Knip M, Constantin ME, Thordal-Christensen H (2014) Trans-kingdom cross-talk: small RNAs on the move. PLOS Genet 10: e1004602. 
Koch A, Biedenkopf D, Furch A, Weber L, Rossbach O, Abdellatef E, Linicus L, Johannsmeier J, Jelonek L, Goesmann A (2016) An RNAi-based control of Fusarium graminearum infections through spraying of long dsRNAs involves a plant passage and is controlled by the fungal silencing machinery. PLOS Pathog 12: e1005901.

Koch A, Kogel KH (2014) New wind in the sails: improving the agronomic value of crop plants through RNAi-mediated gene silencing. Plant Biotechnol J 12: 821-831.

Krasileva KV, Dahlbeck D, Staskawicz BJ (2010) Activation of an Arabidopsis resistance protein is specified by the in planta association of its leucine-rich repeat domain with the cognate oomycete effector. Plant Cell 22: 2444-2458.

Kroj T, Chanclud E, Michel-Romiti C, Grand X, Morel JB (2016) Integration of decoy domains derived from protein targets of pathogen effectors into plant immune receptors is widespread. New Phytol 210: 618-626.

Kusch S, Panstruga R (2017) mlo-Based Resistance: An Apparently Universal "Weapon" to Defeat Powdery Mildew Disease. Mol Plant-Microbe Interact 30: 179-189.

Lacombe S, Rougon-Cardoso A, Sherwood E, Peeters N, Dahlbeck D, Van Esse HP, Smoker M, Rallapalli G, Thomma BPHJ, Staskawicz B (2010) Interfamily transfer of a plant patternrecognition receptor confers broad-spectrum bacterial resistance. Nat Biotechnol 28: 365-369.

Le Roux C, Huet G, Jauneau A, Camborde L, Tremousaygue D, Kraut A, Zhou B, Levaillant M, Adachi H, Yoshioka H, Raffaele S, Berthome R, Coute Y, Parker JE, Deslandes L (2015) A receptor pair with an integrated decoy converts pathogen disabling of transcription factors to immunity. Cell 161: 1074-1088.

Li L, Yu Y, Zhou Z, Zhou J-M (2016) Plant pattern-recognition receptors controlling innate immunity. Sci China Life Sci 59: 878-888.

Liebrand TW, van den Berg GC, Zhang Z, Smit P, Cordewener JH, America AH, Sklenar J, Jones AM, Tameling WI, Robatzek S (2013) Receptor-like kinase SOBIR1/EVR interacts with receptor-like proteins in plant immunity against fungal infection. Proc Natl Acad Sci USA 110: 10010-10015.

Liebrand TW, van den Burg HA, Joosten MH (2014) Two for all: receptor-associated kinases SOBIR1 and BAK1. Trends Plant Sci 19: 123-132.

Liu J, Elmore JM, Lin Z-JD, Coaker G (2011) A receptor-like cytoplasmic kinase phosphorylates the host target RIN4, leading to the activation of a plant innate immune receptor. Cell Host Microbe 9: 137146.

Liu T, Liu Z, Song C, Hu Y, Han Z, She J, Fan F, Wang J, Jin C, Chang J (2012) Chitin-induced dimerization activates a plant immune receptor. Science 336: 1160-1164.

Lozano-Torres JL, Wilbers RH, Gawronski P, Boshoven JC, Finkers-Tomczak A, Cordewener JH, America AH, Overmars HA, Van't Klooster JW, Baranowski L (2012) Dual disease resistance mediated by the immune receptor $\mathrm{Cf}-2$ in tomato requires a common virulence target of a fungus and a nematode. Proc Natl Acad of Sci USA 109: 10119-10124.

Lu F, Wang H, Wang S, Jiang W, Shan C, Li B, Yang J, Zhang S, Sun W (2015) Enhancement of innate immune system in monocot rice by transferring the dicotyledonous elongation factor Tu receptor EFR. J Integr Plant Biol 57: 641-652.

Maekawa T, Kracher B, Vernaldi S, van Themaat EVL, Schulze-Lefert P (2012) Conservation of NLRtriggered immunity across plant lineages. Proc Natl Acad Sci USA 109: 20119-20123.

Malaker P, Barma N, Tiwari T, Collis W, Duveiller E, Singh P, Joshi A, Singh R, Braun H-J, Peterson G (2016) First report of wheat blast caused by Magnaporthe oryzae pathotype triticum in Bangladesh. Plant Dis 100: 2330. 
Maqbool A, Saitoh H, Franceschetti M, Stevenson C, Uemura A, Kanzaki H, Kamoun S, Terauchi R, Banfield M (2015) Structural basis of pathogen recognition by an integrated HMA domain in a plant NLR immune receptor. eLife 4: e08709.

Mendes B, Cardoso S, Boscariol-Camargo R, Cruz R, Mourão Filho F, Bergamin Filho A (2010) Reduction in susceptibility to Xanthomonas axonopodis pv. citri in transgenic Citrus sinensis expressing the rice Xa21 gene. Plant Pathol 59: 68-75.

Mitter N, Worrall E, Robinson K, Li P, Jain R, Taochy C, Fletcher S, Carroll B, Lu G, Xu Z (2017) Clay nanosheets for topical delivery of RNAi for sustained protection against plant viruses. Nat Plants $\mathbf{3}$ : 16207.

Moscou MJ, Bogdanove AJ (2009) A simple cipher governs DNA recognition by TAL effectors. Science 326: 1501-1501.

Mukhtar MS, Carvunis A-R, Dreze M, Epple P, Steinbrenner J, Moore J, Tasan M, Galli M, Hao T, Nishimura MT (2011) Independently evolved virulence effectors converge onto hubs in a plant immune system network. Science 333: 596-601.

Narusaka M, Kubo Y, Hatakeyama K, Imamura J, Ezura H, Nanasato Y, Tabei Y, Takano Y, Shirasu K, Narusaka Y (2013) Interfamily transfer of dual NB-LRR genes confers resistance to multiple pathogens. PLOS ONE 8: e55954.

Nembaware V, Seoighe C, Sayed M, Gehring C (2004) A plant natriuretic peptide-like gene in the bacterial pathogen Xanthomonas axonopodis may induce hyper-hydration in the plant host: a hypothesis of molecular mimicry. BMC Evol Biol 4: 10.

Nishimura MT, Anderson RG, Cherkis KA, Law TF, Liu QL, Machius M, Nimchuk ZL, Yang L, Chung EH, El Kasmi F, Hyunh M, Osborne Nishimura E, Sondek JE, Dangl JL (2017) TIR-only protein RBA1 recognizes a pathogen effector to regulate cell death in Arabidopsis. Proc Natl Acad Sci USA 114: E2053-E2062.

Nunes CC, Dean RA (2012) Host-induced gene silencing: a tool for understanding fungal host interaction and for developing novel disease control strategies. Mol Plant Pathol 13: 519-529.

Okuyama Y, Kanzaki H, Abe A, Yoshida K, Tamiru M, Saitoh H, Fujibe T, Matsumura H, Shenton M, Galam DC (2011) A multifaceted genomics approach allows the isolation of the rice Pia-blast resistance gene consisting of two adjacent NBS-LRR protein genes. Plant J 66: 467-479.

Ortiz D, De Guillen K, Cesari S, Chalvon V, Gracy J, Padilla A, Kroj T (2017) Recognition of the Magnaporthe oryzae effector AVR-Pia by the decoy domain of the rice NLR immune receptor RGA5. Plant Cell 29: 156-168.

Pavan S, Jacobsen E, Visser RG, Bai Y (2010) Loss of susceptibility as a novel breeding strategy for durable and broad-spectrum resistance. Mol Breed 25: 1.

Puchta H (2017) Applying CRISPR/Cas for genome engineering in plants: the best is yet to come. Curr Opin Plant Biol 36: 1-8.

Ranf S, Gisch N, Schäffer M, Illig T, Westphal L, Knirel YA, Sánchez-Carballo PM, Zähringer U, Hückelhoven R, Lee J (2015) A lectin S-domain receptor kinase mediates lipopolysaccharide sensing in Arabidopsis thaliana. Nat Immunol 16: 426-433.

Römer P, Hahn S, Jordan T, Strauß T, Bonas U, Lahaye T (2007) Plant pathogen recognition mediated by promoter activation of the pepper Bs3 resistance gene. Science 318: 645-648.

Römer P, Recht S, Lahaye T (2009) A single plant resistance gene promoter engineered to recognize multiple TAL effectors from disparate pathogens. Proc Natl Acad Sci USA 106: 20526-20531.

Rooney HC, van't Klooster JW, van der Hoorn RA, Joosten MH, Jones JD, de Wit PJ (2005) Cladosporium Avr2 inhibits tomato Rcr3 protease required for Cf-2-dependent disease resistance. Science 308: 1783-1786. 
Sarris PF, Cevik V, Dagdas G, Jones JD, Krasileva KV (2016) Comparative analysis of plant immune receptor architectures uncovers host proteins likely targeted by pathogens. BMC Biol 14: 1.

Sarris PF, Duxbury Z, Huh SU, Ma Y, Segonzac C, Sklenar J, Derbyshire P, Cevik V, Rallapalli G, Saucet SB, Wirthmueller L, Menke FL, Sohn KH, Jones JD (2015) A plant immune receptor detects pathogen effectors that target WRKY transcription factors. Cell 161: 1089-1100.

Saur IM, Kadota Y, Sklenar J, Holton NJ, Smakowska E, Belkhadir Y, Zipfel C, Rathjen JP (2016) NbCSPR underlies age-dependent immune responses to bacterial cold shock protein in Nicotiana benthamiana. Proc Natl Acad Sci USA 113: 3389-3394.

Schaible L, Cannon OS, Waddoups V (1951) Inheritance of resistance to Verticillium wilt in a tomato cross. Phytopathology 41: 986-990.

Schoonbeek Hj, Wang HH, Stefanato FL, Craze M, Bowden S, Wallington E, Zipfel C, Ridout CJ (2015) Arabidopsis EF-Tu receptor enhances bacterial disease resistance in transgenic wheat. New Phytol 206: 606-613.

Schwessinger B, Bahar O, Thomas N, Holton N, Nekrasov V, Ruan D, Canlas PE, Daudi A, Petzold CJ, Singan VR (2015) Transgenic expression of the dicotyledonous pattern recognition receptor EFR in rice leads to ligand-dependent activation of defense responses. PLOS Pathog 11: e1004809.

Segretin ME, Pais M, Franceschetti M, Chaparro-Garcia A, Bos JI, Banfield MJ, Kamoun S (2014) Single amino acid mutations in the potato immune receptor R3a expand response to Phytophthora effectors. Mol Plant-Microbe Interact 27: 624-637.

Shao F, Golstein C, Ade J, Stoutemyer M, Dixon JE, Innes RW (2003) Cleavage of Arabidopsis PBS1 by a bacterial type III effector. Science 301: 1230-1233.

Slootweg EJ, Spiridon LN, Roosien J, Butterbach P, Pomp R, Westerhof L, Wilbers R, Bakker E, Bakker J, Petrescu A-J (2013) Structural determinants at the interface of the ARC2 and leucinerich repeat domains control the activation of the plant immune receptors Rx1 and Gpa2. Plant Physiol 162: 1510-1528.

Song J, Win J, Tian M, Schornack S, Kaschani F, Ilyas M, van der Hoorn RA, Kamoun S (2009) Apoplastic effectors secreted by two unrelated eukaryotic plant pathogens target the tomato defense protease Rcr3. Proc Natl Acad Sci USA 106: 1654-1659.

Stam R, Scheikl D, Tellier A (2016) Pooled enrichment sequencing identifies diversity and evolutionary pressures at NLR resistance genes within a wild tomato population. Genome Biol Evol 8: 15011515.

Steinbrenner AD, Goritschnig S, Staskawicz BJ (2015) Recognition and activation domains contribute to allele-specific responses of an Arabidopsis NLR receptor to an oomycete effector protein. PLOS Pathog 11: e1004665.

Sun Y, Li L, Macho AP, Han Z, Hu Z, Zipfel C, Zhou J-M, Chai J (2013) Structural basis for flg22-induced activation of the Arabidopsis FLS2-BAK1 immune complex. Science 342: 624-628.

Tameling WI, Elzinga SD, Darmin PS, Vossen JH, Takken FL, Haring MA, Cornelissen BJ (2002) The tomato $R$ gene products I-2 and MI-1 are functional ATP binding proteins with ATPase activity. Plant Cell 14: 2929-2939.

Tang J, Han Z, Sun Y, Zhang H, Gong X, Chai J (2015) Structural basis for recognition of an endogenous peptide by the plant receptor kinase PEPR1. Cell Res 25: 110-120.

Thomma BPHJ, Nürnberger T, Joosten MH (2011) Of PAMPs and effectors: the blurred PTI-ETI dichotomy. Plant Cell 23: 4-15.

Tripathi JN, Lorenzen J, Bahar O, Ronald P, Tripathi L (2014) Transgenic expression of the rice Xa21 pattern-recognition receptor in banana (Musa sp.) confers resistance to Xanthomonas campestris pv. musacearum. Plant Biotechnol J 12: 663-673. 
Van Der Biezen EA, Jones JD (1998) Plant disease-resistance proteins and the gene-for-gene concept. Trends Biocheml Sci 23: 454-456.

van Esse HP, van't Klooster JW, Bolton MD, Yadeta KA, van Baarlen P, Boeren S, Vervoort J, de Wit PJ, Thomma BPHJ (2008) The Cladosporium fulvum virulence protein Avr2 inhibits host proteases required for basal defense. Plant Cell 20: 1948-1963.

van Schie CC, Takken FL (2014) Susceptibility genes 101: how to be a good host. Ann Rev Phytopathol 52: 551-581.

Vleeshouwers VG, Oliver RP (2014) Effectors as tools in disease resistance breeding against biotrophic, hemibiotrophic, and necrotrophic plant pathogens. Mol Plant-Microbe Interact 27: 196-206.

Wang A, Krishnaswamy S (2012) Eukaryotic translation initiation factor 4E-mediated recessive resistance to plant viruses and its utility in crop improvement. Mol Plant Pathol 13: 795-803.

Wang G-F, Ji J, Farid E-K, Dangl JL, Johal G, Balint-Kurti PJ (2015) Molecular and functional analyses of a maize autoactive NB-LRR protein identify precise structural requirements for activity. PLOS Pathog 11: e1004674.

Wang L, Albert M, Einig E, Fürst U, Krust D, Felix G (2016) The pattern-recognition receptor CORE of Solanaceae detects bacterial cold-shock protein. Nat Plants 2: 16185.

Wang M, Jin H (2017) Spray-induced gene silencing: a powerful innovative strategy for crop protection. Trends Microbiol 25: 4-6.

Wang M, Thomas N, Jin H (2017) Cross-kingdom RNA trafficking and environmental RNAi for powerful innovative pre-and post-harvest plant protection. Curr Opin Plant Biol 38: 133-141.

Wang M, Weiberg A, Lin F-M, Thomma BP, Huang H-D, Jin H (2016) Bidirectional cross-kingdom RNAi and fungal uptake of external RNAs confer plant protection. Nat Plants 2: 16151.

Wang X, Richards J, Gross T, Druka A, Kleinhofs A, Steffenson B, Acevedo M, Brueggeman R (2013) The rpg4-mediated resistance to wheat stem rust (Puccinia graminis) in barley (Hordeum vulgare) requires Rpg5, a second NBS-LRR gene, and an actin depolymerization factor. Mol Plant-Microbe Interact 26: 407-418.

Wang Y, Nsibo DL, Juhar HM, Govers F, Bouwmeester K (2016) Ectopic expression of Arabidopsis Ltype lectin receptor kinase genes LecRK-I.9 and LecRK-IX.1 in Nicotiana benthamiana confers Phytophthora resistance. Plant Cell Rep 35: 845-855.

Weiberg A, Jin H (2015) Small RNAs-the secret agents in the plant-pathogen interactions. Curr Opin Plant Biol 26: 87-94.

Weßling R, Epple P, Altmann S, He Y, Yang L, Henz SR, McDonald N, Wiley K, Bader KC, Gläßer C (2014) Convergent targeting of a common host protein-network by pathogen effectors from three kingdoms of life. Cell Host Microbe 16: 364-375.

Witek K, Jupe F, Witek AI, Baker D, Clark MD, Jones JD (2016) Accelerated cloning of a potato late blight-resistance gene using RenSeq and SMRT sequencing. Nat Biotechnol 34: 656-660.

Wu C-H, Krasileva KV, Banfield MJ, Terauchi R, Kamoun S (2015) The "sensor domains" of plant NLR proteins: more than decoys? Front Plant Sci 6: 134.

Xiao N, Wu Y, Pan C, Yu L, Chen Y, Li Y, Dai Z, Liang C, Li A (2016) Improving of rice blast resistances in japonica by pyramiding major R genes. Front Plant Sci 7: 1918.

Yuan B, Zhai C, Wang W, Zeng X, Xu X, Hu H, Lin F, Wang L, Pan Q (2011) The Pik-p resistance to Magnaporthe oryzae in rice is mediated by a pair of closely linked CC-NBS-LRR genes. Theor Appl Genet 122: 1017-1028.

Zeng X, Tian D, Gu K, Zhou Z, Yang X, Luo Y, White FF, Yin Z (2015) Genetic engineering of the Xa10 promoter for broad-spectrum and durable resistance to Xanthomonas oryzae pv. oryzae. Plant Biotechnol J 13: 993-1001. 


\section{Chapter 7}

Zhang H, Wang S (2013) Rice versus Xanthomonas oryzae pv. oryzae: a unique pathosystem. Curr Opin Plant Biol 16: 188-195.

Zhang T, Jin Y, Zhao J-H, Gao F, Zhou B-J, Fang Y-Y, Guo H-S (2016) Host-induced gene silencing of the target gene in fungal cells confers effective resistance to the cotton wilt disease pathogen Verticillium dahliae. Mol Plant 9: 939-942.

Zhang Z, Thomma BP (2013) Structure-function aspects of extracellular leucine-rich repeat-containing cell surface receptors in plants. J Integr Plant Biol 55: 1212-1223.

Zhu S, Li Y, Vossen JH, Visser RG, Jacobsen E (2012) Functional stacking of three resistance genes against Phytophthora infestans in potato. Transgenic Res 21: 89-99. 


\section{SUMMARY}

Plant-pathogenic microbes secrete effector molecules to establish disease on their hosts, whereas plants in turn employ immune receptors to try and intercept such effectors in order to prevent pathogen colonization. Based on structure and subcellular location, immune receptors fall into two major classes; cell surfacelocalized receptors that comprise receptor kinases (RKs) and receptor-like proteins (RLPs) that monitor the extracellular space, and cytoplasm-localized nucleotidebinding domain leucine-rich repeat receptors (NLRs) that survey the intracellular environment. Chapter 1 describes conceptual advances explaining the plant innate immunity system, and advances in our understanding of genetic resistance against Verticillium wilt, with emphasis on Verticillium resistance mediated by the Ve locus. In tomato (Solanum lycopersicum), race-specific resistance to Verticillium wilt is governed by the extracellular leucine-rich repeat (eLRR)-containing RLP-type cell surface receptor Ve1, that is encoded by the Ve locus, through recognition of the effector protein Ave1 that is secreted by race 1 strains of Verticillium spp.. Homologues of Verticillium dahliae Ave1 (VdAve1) are found in plants and in a number of plant pathogenic microbes, and some of these VdAve1 homologues are recognized by tomato Ve1. The research described in this thesis aims to characterize the role of the tomato cell surface-localized immune receptor Ve1, and its homologues in various other plant species, in Verticillium wilt resistance.

Research on effectors secreted by pathogens during host attack has dominated the field of molecular plant-microbe interactions over recent years. Chapter 2 reviews the current status of research on how soil-borne pathogens employ secreted effector molecules to support host colonization. Most well-studied effectors are proteinaceous molecules, but also non-canonical effectors, such as metabolites and small RNA molecules, have been described. Soil-borne pathogens live in a complex and sophisticated below-ground environment. In addition to targeting of plant components, part of the effector catalogue may be required for self-defense against, and targeting of, other microbiome partners, including antagonists and competitors.

It has previously been demonstrated that many eLRR-containing cell surfacelocalized immune receptors often recognize short peptide sequence stretches as epitopes of their pathogen ligands. In Chapter 3, we aimed to identify a minimal 
motif in VdAve1 that is necessary and sufficient for recognition by the tomato cellsurface immune receptor Ve1. Epitope prediction, serial deletion mutants, domain swaps, synthetic peptides, chimeric protein and three-dimensional modelling were exploited to identify which part of the effector protein VdAve1 is recognized by Ve1. Our analyses revealed that the $\mathrm{C}$-terminus as well as the $\mathrm{N}$-terminus are individually required, but not sufficient, to activate Ve1-mediated immunity. A three-dimensional model of the VdAve1 protein explains why both termini are required, by showing that both termini co-localize on a surface-exposed patch of the VdAve1 protein. We propose that a surface-exposed patch of the VdAve1 protein that is composed of co-localized $\mathrm{N}$ - and $\mathrm{C}$ - termini is recognized by the tomato cell-surface immune receptor Ve1.

In Chapter 4, we describe the cloning and characterization of tomato Ve1 homologues within and outside of the Solanaceae family, and demonstrate that particular Ve1 homologues from tobacco (Nicotiana glutinosa; NgVe1), potato (Solanum tubersoum; StuVe1), wild eggplant (Solanum torvum; StoVe1) and hop (Humulus lupulus; HLVe1-2A) serve as functional Ve1 homologues by conferring resistance to race $1 \mathrm{~V}$. dahliae through recognition of VdAve1, implying that functional Ve1 homologues are conserved across plant species within and outside the Solanaceae.

It was previously demonstrated that heterologous expression of tomato Ve1 in the model plant Arabidopsis thaliana that does not normally respond to the Verticillium effector Ave1, leads to Verticillium wilt resistance against race 1 Verticillium spp. In Chapter 5 we investigated whether tomato Ve1 can confer Verticillium wilt resistance when expressed in the crop species tobacco (Nicotiana tabcum) and cotton (Gossypium hirsutum). Stable transgenic tobacco and cotton lines constitutively expressing tomato $V e 1$ were generated. Expression of tomato $V e 1$ in tobacco resulted in stunted growth, while cotton plants expressing Ve1 did not show growth retardation. Ve1-transgenic tobacco and cotton plants displayed enhanced resistance against Verticillium wilt in an Ave1-dependent manner. This demonstrates that the functionality of tomato Ve1 in resistance against Verticillium wilt through recognition of the Verticillium effector Ave1 is retained after transfer to tobacco and cotton. 
Host-induced gene silencing (HIGS) is an RNA interference (RNAi)-based process in which small RNAs are produced by the host plant to target parasite transcripts. HIGS has recently emerged as a promising strategy for the improvement of plant resistance against pathogens by silencing genes that are essential for these pathogens. In Chapter 6, we assessed whether HIGS can be deployed to inhibit Verticillium wilt disease by silencing previously identified virulence genes of $V$. dahliae (encoding Ave1, Sge1 and NLP1 respectively) through the host plants tomato and A. thaliana. Firstly, we established a Tobacco rattle virus (TRV)-mediated HIGS assay through targeting of V. dahliae Ave1 transcripts in Ve1 tomato plants, and further used this approach to assess whether HIGS against $V$. dahliae in tomato could be established through TRV constructs targeting previously identified $V$. dahliae virulence factors. We also investigated whether HIGS against $V$. dahliae could be established in transgenic A. thaliana plants through hairpin RNAbased RNAi constructs targeting transcripts of the same previously identified $V$. dahliae virulence genes. Our results clearly show that plants transiently (in tomato) or stably (in A. thaliana) expressing RNAi constructs targeting transcripts of genes that are essential for $V$. dahliae pathogenicity can become protected from Verticillium wilt disease.

In Chapter 7, the major results described in this thesis are discussed and placed in a broader perspective. Taking the findings of this thesis into account, a current overview for the improvement of plant disease resistance through the deployment of plant immune receptors is presented, and future directions to enhance disease resistance in crops through the exploitation of immune receptors and through using other approaches, such as editing of host susceptibility genes or exploitation of sRNA trafficking-based disease control strategies, is discussed. 



\section{ACKNOWLEDGEMENTS}

Time flies! My PhD journey has come to the end. I would not have been able to reach the end of this journey without the help and support from supervisors, colleagues, friends and family. Here I would like to take the opportunity to thank everyone who made my PhD journey unforgettable.

First and foremost, I would like to thank my promotor and daily supervisor Prof. Bart Thomma. Dear Bart, thank you for offering me the opportunity to conduct my PhD study in your group. Ever since I joined your group for my PhD study I have been inspired by your passion and dedication to science. I appreciate your extraordinary supervision, but also your guidance in making my presentation slides, improving my manuscripts and my PhD thesis. I really appreciate your efforts to help me improve my English skills. I have learned a lot from you, both in science and life. I am happy to be one of your PhD students. Thanks a lot!

Furthermore, my sincere thanks go to my second promotor Prof. Pierre de Wit. Dear Pierre, thank you for allowing me to work in the Laboratory of Phytopathology when you were the head of the laboratory. You are a great mentor, and your enthusiasm and profound insights into science impressed me a lot! Definitely you have set a perfect example of being a scientist.

My special acknowledgements go to Dr. Guodong Wang (王国栋) and Dr. Zhao Zhang (钊哥) for your help, suggestions, support and inspiration over the past five years. Without your help, I would not have been able to come to Wageningen for my PhD study. I appreciate your great help, both in study and life.

I thank the (former) members of Verticillium group; Peter van Esse, Mireille van Damme, Dirk-Jan Valkenburg, Andrea Sánchez Vallet, Anja Kombrink, Luigi Faino, David Cook, Ermis Yanes Paz, Mojtaba Keykha Saber, Eduardo Rojas Padilla, Longfu Zhu (朱龙付老师), Baolong Zhang (张保龙老师), Tingli Liu (刘廷利), Hanna Rövenich, Jordi Boshoven, Michael Seidl, Xiaoqian Shi (史晓倩), Grardy van den Berg, Jasper Depotter, Malaika Ebert, Nick Snelders, Martin Kramer, Hesham Gibriel, Jasper Vermeulen, Katharina Hanika, Luis Rodriguez Moreno. I especially thank Jinling Li (金岭) and Hui Tian (田卉) for their great help in the laboratory during 
times when I was not in Wageningen. I also thank all other colleagues of the Laboratory of Phytopathology. I have enjoyed very much to work with all of you in this laboratory with its friendly and welcoming atmosphere.

I am happy to have met so many friends in the Netherlands who brought so much fun and happiness in Wageningen, as well as outside. Thank all of you; Guozhi Bi (老毕), Yu Du (杜羽) \& Guangzhao Liu (刘光照), Yan Wang (孙太) \& Yinyin Sun (孙 总), Chunxu Song (春哥) \& Wei Qin (伟哥), Lisha Zhang (丽莎) \& Chenlei Hua (辰雷), Yidong Wang (依冬), Yiqian Fu (小卷丹) \& Tonggao Ren (通哥), Yongfu Tang (老唐) \& Jiacui Chen (家翠), Fahui Liu (辉哥) \& Wen Zhang (辉嫂), Lingmin Tian (田灵敏), Jinbin Wu (锦斌), Shuqing Yang (杨叔青), Xiaoxue Sun (晓雪), Shanshan Qi (珊珊), Xiaolin Wu (小老板), Kaile Sun (凯乐), Mengyang Liu (刘梦洋), Peirong Li (佩荣), Lujun Zhang (章老师), Xiao Lin (四少), Xu Cheng (旭哥), Feng Zhu (Jerry), Yue Lu (鲁玥), Juncai Chen (才哥), Huichen Li (虎臣), Lihe Xu (徐丽鹤) \& Changkai Qiao (乔 昌凯), Xinyu Yang (杨新宇), Dongli Yu (东立), Haiyan Ma (马海燕), Peng Wang (王 鹏), Xiaomei Yang (杨晓梅), Hongming Zhang (张宏鸣), Zhichun Yan (严志纯), Lu Luo (罗露), Xixi Minqian (希䂀), Rongran Ji (季永然), Yuchen Long (龙宇尘), Yuanyuan Zhang (张媛园), Defeng Shen (申德峰), Guiling Ren (任贵玲), Tingting Xiao (婷婷), Fengjiao Bu (凤娇), Wenkun Zhou (周文坤), Yujuan Du (杜玉鹃), Tian Zeng (曾添), Jingmeng Wang (王菁梦), Hao Hu (胡吴), Huayi Li (华一), Xuexue Shen (申雪雪), Yajun Wang (亚军) \& Wen Cui (崔文), Mengjing Sun (孙梦婧) \& Wei Du (杜伟), Yunmeng Zhang (张云梦), Juan Du (杜鹃), Dongli Gao (高冬丽), Chunzhi Zhang (张春芝), Bo Wang (王波), Wei Song (宋伟), Wenbo Jiang (江老师), Changchun Wang (王老师), Junwei Liu (刘军伟) \& Hanzi He (何菉子), Bing Bai (白 兵), Xuan Xu (许荁), Zhen Wei (魏珍), Shuhang Wang (王舒航), Jiao Long (龙蛟) \& Ya Wang (王亚), Lina Zhang (张丽娜), Yingxue Ren (任映雪), Min Chen (陈敏), Chunyue Zhang (张春月), Yifan Zhu (凡哥), Jie Lian (廉洁), Zhongnan Chen (陈中南), Hailin Zhang (Roy), Wenbiao Shi (标哥), Jing Tang (静哥), Haibo Lu (路海博), Chen Zhang (张晨), Libin Zhou (周礼斌), Lin Tian (田林), Chen Zhang (张陈), Xiaotang Di (邸晓棠), Lingxue Cao (曹凌雪), Meiliang Zhou (周美亮), Tao Li (李涛), Dong Hu (胡 栋), Yuanchuan Zhang (张塬川), Loretto van der Velde.

Finally my sincere appreciations go to my family. I would like to express my great gratitude to my mother for supporting my decisions and her endless love. Great 
thanks to my sister Bingjun Song (宋炳君) and brother-in-law Bo Bai (白波) for taking care of our mother. I also would like to thank my cute niece Yutong Bai (白钰 粀) and nephew Jiaming Bai (白嘉铭) for the great fun when I was on holiday in China. This thesis is dedicated to my family, and also in memory of my father who passed away in a car accident seven years ago.

\section{Yin Song 宋银}

26 ${ }^{\text {th }}$ September, 2017

Radix building, Wageningen 



\section{CURRICULUM VITAE}

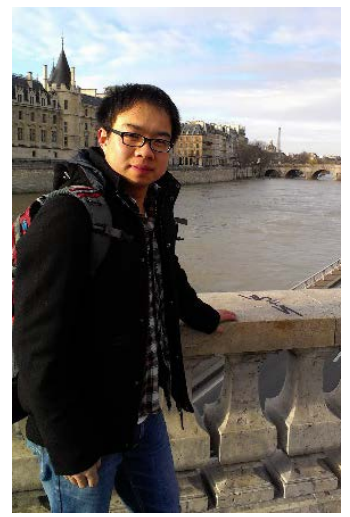

Yin Song (宋银) was born on July 16 $6^{\text {th }}, 1985$ in Hanzhong, Shaanxi province, China. In 2008, he finished his BSc study in Plant Sciences at Southwest Forestry University, Kunming, China. One year later, Yin was enrolled in a threeyear master training program at Shaanxi Normal University, Xi'an, China. He performed his MSc thesis project on metabolic engineering of pharmaceutical compounds in the medicinal plant Salvia miltiorrhiza, under the supervision of Prof. Zhezhi Wang. During his MSc, he worked as a volunteer research assistant in the group of Receptor-like Protein/Kinase Function and Peptide Signaling, led by Dr. Guodong Wang. In 2012 he was awarded a PhD fellowship from the China Scholarship Council (CSC) to join the Verticillium group at the Laboratory of Phytopathology, Wageningen University, the Netherlands, under the supervision of Prof. dr. Bart Thomma and Prof. dr. Pierre de Wit. His PhD research resulted in the publication of this thesis entitled: "Recognition of Verticillium effector Ave1 by tomato immune receptor Ve1 mediates Verticillium resistance in diverse plant species". 


\section{List of publications}

Yin Song\#, Zhao Zhang\#, Jordi C. Boshoven, Hanna Rovenich, Michael F. Seidl, Jernej Jakše, Karunakaran Maruthachalam, Chun-Ming Liu, Krishna V. Subbarao, Branka Javornik and Bart P.H.J. Thomma (2017) Tomato immune receptor Ve1 recognizes surface-exposed co-localized N- and C-termini of Verticillium dahliae effector Ave1. bioRxiv: https://doi.org/10.1101/103473.

Jordi C. Boshoven, Malaika Ebert\$, Yin Song $\$$, Hanna Rovenich, Eduardo RojasPadilla, Melvin D. Bolton and Bart P.H.J. Thomma (2017) Homologs of Verticillium dahliae effector Ave1 contribute to virulence of various fungal plant pathogens. (submitted).

Yin Song, Linlin Liu, Yidong Wang, Dirk-Jan Valkenburg, Xianlong Zhang, Longfu Zhu and Bart P.H.J. Thomma (2017) Transfer of tomato immune receptor Ve1 confers Ave1-dependent Verticillium resistance in tobacco and cotton. Plant Biotechnology Journal. DOI: 10.1111/pbi.12804.

Luis Rodriguez-Moreno\#, Yin Song\# and Bart P.H.J. Thomma (2017) Transfer and engineering of immune receptors to improve recognition capacities in crops. Current Opinion in Plant Biology 38: 42-49.

Yin Song, Zhao Zhang, Michael F. Seidl, Aljaz Majer, Jernej Jakse, Branka Javornik and Bart P.H.J. Thomma (2017) Broad taxonomic characterization of Verticillium wilt resistance genes reveals an ancient origin of the tomato Ve1 immune receptor. Molecular Plant Pathology 18: 195-209.

Yin Song and Bart P.H.J. Thomma (2016) Host-induced gene silencing compromises Verticillium wilt in tomato and Arabidopsis. Molecular Plant Pathology. DOI: 10.1111/mpp.12500.

Zhao Zhang, Yin Song, Chun-Ming Liu and Bart P.H.J. Thomma (2014) Mutational analysis of the Ve1 immune receptor that mediates Verticillium resistance in tomato. PLoS ONE 9:e99511. doi:10.1371/journal.pone.0099511.

Emilie F. Fradin\#, Zhao Zhang\#, Hanna Rövenich\$, Yin Song\$, Thomas W.H. Liebrand, Laura Masini, Grardy C.M. van den Berg, Matthieu H.A.J. Joosten and Bart P.H.J. 
Thomma (2014) Functional analysis of the tomato immune receptor Ve1 through domain swaps with its non-functional homolog Ve2. PLOS ONE 9: e88208. doi:10.1371/journal.pone. 0088208.

Donghao Wang\#, Yin Song\#, Yuqin Chen, Wei Yao, Zhen Li, Wenchao Liu, Sisi Yue and Zhezhi Wang (2013) Metabolic pools of phenolic acids in Salvia miltiorrhiza are enhanced by co-expression of Antirrhinum majus Delila and Rosea1 transcription factors. Biochemical Engineering Journal 74: 115-120.

Donghao Wang, Wei Yao, Yin Song, Wenchao Liu, Zhezhi Wang (2012) Molecular characterization and expression of three galactinol synthase genes that confer stress tolerance in Salvia miltiorrhiza. Journal of Plant Physiology 169: 18381848.

\#,\$ equal contribution 



\title{
Education Statement of the Graduate School Experimental Plant Sciences
}

\author{
Issued to: Yin Song \\ Date: 23 October 2017 \\ Group: Laboratory of Phytopathology \\ University: $\quad$ Wageningen University \& Research
}

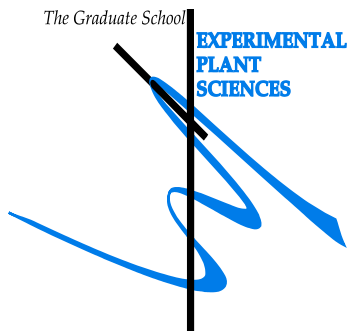

1) Start-up phase

$\underline{\text { date }}$

- First presentation of your project

Title: Interactions of Ve1 and Ave1 homologues: From recognition to resistance

20 Dec 2013

- Writing or rewriting a project proposal

Title: The genetics of receptor-like protein (RLP) Ve-mediated signalling in defense against Verticillium in

Arabidopsis and tomato

Oct 2012

- Writing a review or book chapter

Title: Transfer and engineering of immune receptors to improve recognition capacities in crops.

Current Opinion in Plant Biology 2017, 38:42-49

May 2017

- MSc courses

Laboratory use of isotopes

\section{2) Scientific Exposure}

- EPS PhD student day, Amsterdam, NL

EPS PhD student day, Leiden, NL

EPS PhD student day 'Get2Together', Soest, NL

5th European Plant Science Retreat for PhD students, Ghent, Belgium

- EPS theme symposia

EPS theme 2 symposium 'Interactions between Plants and Biotic Agents',

\& Willie Commelin Scholten day, Utrecht, NL

EPS theme 2 symposium 'Interactions between Plants and Biotic Agents',

\& Willie Commelin Scholten day, Amsterdam, NL

24 Jan 2013

EPS theme 2 symposium 'Interactions between Plants and Biotic Agents',

\& Willie Commelin Scholten day, Leiden, NL

EPS theme 2 symposium 'Interactions between Plants and Biotic Agents',

\& Willie Commelin Scholten day, Wageningen, NL

25 Feb 2014

22 Jan 2016

$23 \operatorname{Jan} 2017$

- National meetings (e.g. Lunteren days) and other national platforms

Annual meeting 'Experimental Plant Sciences', Lunteren, NL

Annual meeting 'Experimental Plant Sciences', Lunteren, NL

14-15 Apr 2014

Annual meeting 'Experimental Plant Sciences', Lunteren, NL

Annual meeting 'Experimental Plant Sciences', Lunteren, NL

- Seminars (series), workshops and symposia

Mini-symposium: Frontiers in Plant Morphogenesis (Chun-Ming Liu, Sidney Shaw, Dolf Weijers, Viktor Žárský)

13 Nov 2012

EPS flying seminar Prof. Ralph Panstruga: Comparative pathogenomics of powdery mildew fungi: chasing the molecular secrets of obligate biotrophy and fungal pathogenesis 
Seminar Prof. dr. Brian Staskawicz: Effector-Targeted Breeding for Durable Disease Control of Xanthomonas
diseases in Tomato and Cassava

Seminar Dr. Pieter Dorrestein: A "GoogleAMP"-type molecular view of microbes-from culture to people

21 May 2013

Seminar: How to write a world-class paper

22 Aug 2013

Seminar Prof. Jiayang Li: Understanding the molecular mechanisms underlying rice tillering

17 Oct 2013

15 Nov 2013

Seminar Prof. Jos Raaijmakers: Back to the Roots: exploring and exploiting the plant microbiome

07 Jan 2014

Seminar Dr. Jeroen Mesters: From protein solution to single crystal X-ray diffraction: Chitin binding by LysM domains at atomic resolution

Seminar Prof. Jane Parker: Reprogramming cells for defence in plant innate immunity

31 Mar 2014

09 Apr 2014

Seminar Dr. Frank van Breusegem: Plant metacaspases

Seminar Prof. Sophien Kamoun: Genome and effector evolution in the Irish potato famine pathogen lineage

09 Apr 2014

28 May 2014

Farewell symposium Prof. dr. Pierre de Wit: Fungal plant pathogens and the plant immune system

Seminar Prof. Yuanchao Wang: Dissecting the interaction between Phytophthora sojae and soybean:

making sense out of signalling and effectors

Seminar Prof. Hanhui Kuang 'Using the Nicotiana-TMV system to study resistance gene evolution

and plant genome stability'

Seminar Dr. Michael Freitag: Chromatin structure controls centromeres and secondary metabolism

in filamentous fungi

Phytopathology Lab. mini-symposium (Erik Limpens, Tijs Ketelaar, Eric Schranz, Frank Takken \& Weixing Shan)

EPS symposium: Omics Advances for Academia and Industry - Towards True Molecular Plant Breeding

Seminar Prof. Nathalia Requena: Plant-fungal communication in the arbusucular mycorrhizal symbiosis

Seminar Prof. Jiming Jiang: Structure and evolution of centromeres: lessions learned from plants

Phytopathology Lab. symposium: Crop Pathology and Plant-Microbe Interactions

Plant sciences seminar: Into the Battle between Plants and Viruses, but what about EVEs?

EPS flying seminar Prof. Gero Steinberg: Long-distance endosome trafficking drives fungal effector production during plant infection

EPS flying seminar Prof. Jane Parker: Plant intracellular immunity: evolutionary and molecular underpinnings

05 Jun 2014

16 Jul 2014

11 Sep 2014

21 Oct 2014

24 Nov 2014

11 Dec 2014

30 Jan 2015

01 Apr 2015

08 May 2015

12 May 2015

05 Jun 2015

21 Jan 2016

Seminar Prof. dr. Douglas Mitchell: Genomics-enabled natural products discovery

31 Mar 2016

Seminar Dr. Pierre-Marc Delaux: Evolution of symbiotic gene networks in land plants

Seminar Prof. Caitilyn Allen: How Ralstonia solanacearum succeeds in plant xylem vessels

08 Apr 2016

29 Apr 2016

20 Jun 2016

Seminar Prof. Wenbo Ma: Effectors as molecular probes to understand pathogenesis

Seminars Dr. Edze Westra and Prof. Jennifer Doudna: Rewriting our genes? CRISPR-CAS systems as tools for genome editing

1st WURomics symposium: Technology-Driven Innovation for Plant Breeding

30 Sep 2016

15 Dec 2016

08 Feb 2017

Farwell symposium Prof. Ton Bisseling: The Undergrond Labyrinth: Roots, Friends and Foes

01 Mar 2017

Phytopathology Lab. mini-symposium on applied Phytopathology: From the lab to the field

- Seminar plus

- International symposia and congresses

COST SUSTAIN workshop: Pathogen-informed crop improvement, Wageningen, NL

08-10 Apr 2015

The 3rd COST SUSTAIN Anuual Conference, Banyus Sur Mer, France

17-19 Feb 2016

12th International Verticillium Symposium, Ljubljana, Slovenia

06-09 Oct 2016

The 5th International Conference on Biotic Plant Interactions, Xiamen, China

17-21 Aug 2017

\section{- Presentations}

Poster: Annual meeting 'Experimental Plant Sciences', Lunteren, NL

14-15 Apr 2014

Talk: COST SUSTAIN workshop, Wageningen, NL

09 Apr 2015

09 Oct 2016

Talk: 12th International Verticillium Symposium, Ljubljana, Slovenia

\section{- IAB interview}

\section{- Excursions}

Excursion to Rijk Zwaan, De Lier, NL

Excursion to Enza Zaden, Enkhuizen, NL

12 Jun 2015 


\section{EPS courses or other PhD courses}

7th Utrecht PhD summer school on Environmental Signaling, Utrecht, NL

- Journal club

Member of literature discussion at Verticillium group in Phytopathology Lab.

\section{4) Personal development}

date

\section{- Skill training courses}

Practical English, Wageningen, NL

English IETLS training, Wageningen, NL

Private English training course, Wageningen, NL

- Organisation of PhD students day, course or conference

- Membership of Board, Committee or PhD council

Herewith the Graduate School declares that the $\mathrm{PhD}$ candidate has complied with the educational requirements set by the Educational Committee of EPS which comprises of a minimum total of 30 ECTS credits

${ }^{*}$ A credit represents a normative study load of 28 hours of study. 
The research was performed at the Laboratory of Phytopathology, Wageningen University \& Research, the Netherlands. Yin Song was financially sponsored by a $\mathrm{PhD}$ fellowship from the China Scholarship Council (CSC). Financial support from Wageningen University for printing this thesis is gratefully acknowledged.

Cover design: Fahui Liu

Layout: Yin Song

Printed by: GVO drukkers \& vormgevers B.V. Ede (NL) | gvo.nl 